\title{
Onset and Progression of Neurodegeneration in Mouse Models for Defective Endocytosis
}

\section{Dissertation}

\author{
for the award of the degree \\ "Doctor rerum naturalium" \\ of the Georg-August-Universität Göttingen
}

within the doctoral program Sensory and Motor Neuroscience

submitted by

Christine Melina Rostosky

born in Sickte, Germany

Göttingen, September 2018 
Thesis Advisory Committee

Dr. Ira Milosevic

$1^{\text {st }}$ referee

Synaptic Vesicle Dynamics

European Neuroscience Institute

Göttingen, Germany

Prof. Dr. Thomas Bayer

Molecular Psychiatry

$2^{\text {nd }}$ referee

University Medical Center

Göttingen, Germany

Prof. Dr. Tiago Outeiro

Neurodegeneration and Restorative

Research

University Medical Center

Göttingen, Germany

\section{Further members of the Examination Board}

Prof. Dr. Nils Brose

Molecular Neurobiology

Max Planck Institute of Experimental

Medicine

Göttingen, Germany

Dr. Sonja Wojcik

Molecular Neurobiology

Max Planck Institute of Experimental

Medicine

Göttingen, Germany

Dr. Nuno Raimundo

Institute of Cellular Biochemistry

Department of Biochemistry

University Medical Center

Göttingen, Germany 
"Still 'round the corner there may wait a new road or a secret gate" - J.R.R. Tolkien 


\section{Affidavit}

I hereby declare that the dissertation with the title "Onset and Progression of Neurodegeneration in Mouse Models for Defective Endocytosis" was written independently and with no other aids or sources than quoted.

Göttingen, September 2018

Christine Melina Rostosky

Part of the work used for this thesis has been peer-reviewed and published in the following manuscripts:

Murdoch, J. D., Rostosky, C. M., Gowrisankaran, S., Arora, A. S., Soukup, S. F., Vidal, R., Capece, V., Freytag, S., Fischer, A., Verstreken, P., Bonn, S., Raimundo, N., Milosevic, I. (2016). Endophilin-A Deficiency Induces the Foxo3a-Fbxo32 Network in the Brain and Causes Dysregulation of Autophagy and the Ubiquitin-Proteasome System. Cell Reports, 17(4), 1071-1086

Rostosky, C. M., \& Milosevic, I. (2018). Gait Analysis of Age-dependent Motor Impairments in Mice with Neurodegeneration. J. Vis. Exp., 136, e57752. 


\section{Table of Contents}

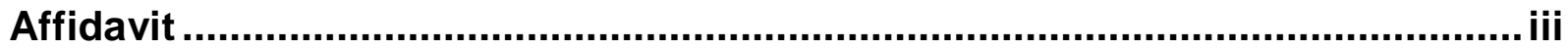

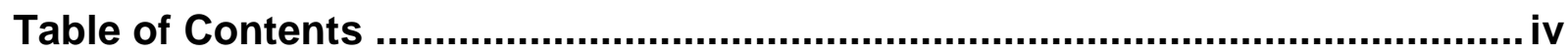

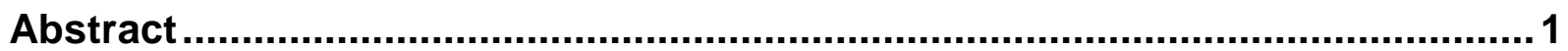

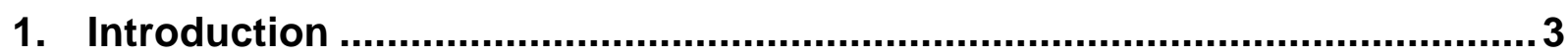

1.1. The central nervous system and neurodegenerative diseases .................. 3

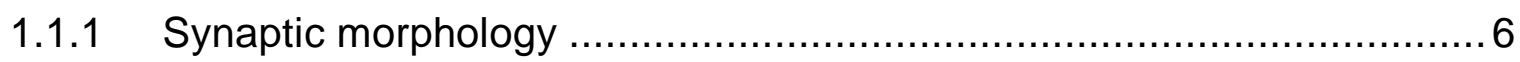

1.2. Endocytosis - an essential process for maintaining synaptic activity .........10

1.2.1 Clathrin-mediated endocytosis ................................................. 10

1.2.2 Ultrafast endocytosis .......................................................... 12

1.2.3 Activity-dependent bulk endocytosis ......................................... 12

1.2.4 Kiss-and-run endocytosis ....................................................... 13

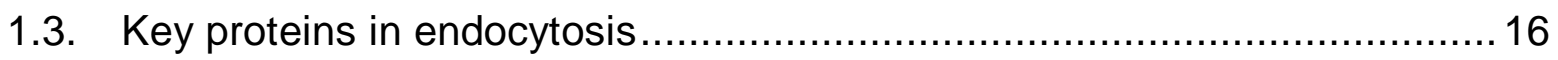

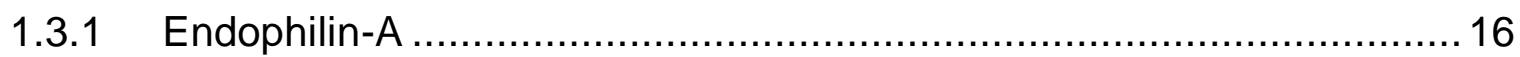

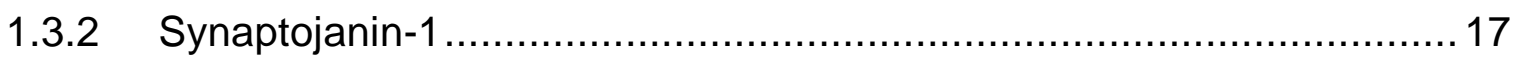

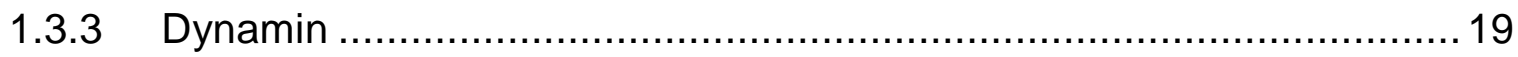

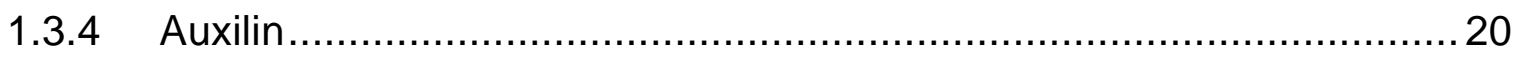

1.4. Mouse models of defective endocytosis ........................................... 21

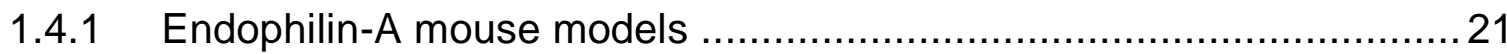

1.4.2 Synaptojanin-1 mouse models …............................................. 23

1.4.3 The dynamin-1,3 mouse model................................................. 24

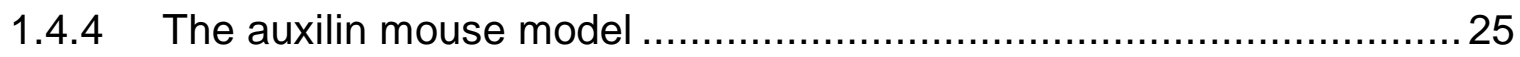

1.5. Protein degradation as an important part of proteostasis .........................27

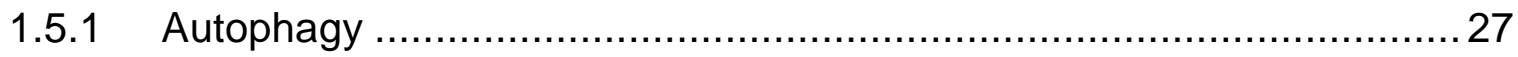

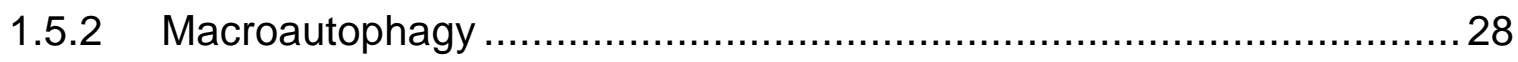




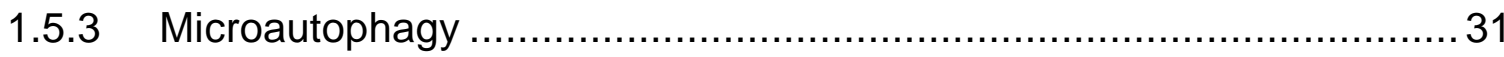

1.5.4 Chaperone mediated autophagy …............................................. 31

1.5.5 The Ubiquitin-Proteasome-System …......................................... 33

1.6. Fbxo32, an E3 Ubiquitin-Ligase involved in autophagy .......................... 37

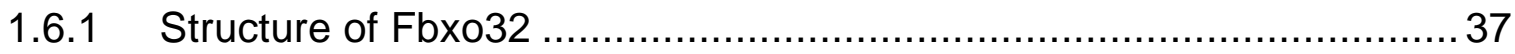

1.6.2 Role and targets of Fbxo32 in proteasomal degradation .....................39

1.6.3 Fbxo32 as a mediator between autophagy and the UPS ................... 41

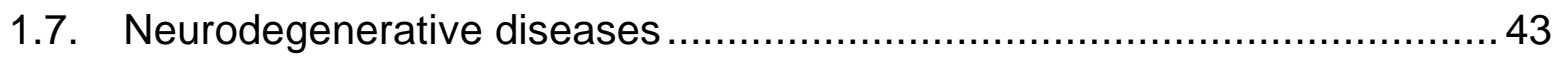

1.7.1 Defective endocytosis and neurodegeneration............................... 44

1.7.2 Autophagy impairments and neurodegeneration .............................46

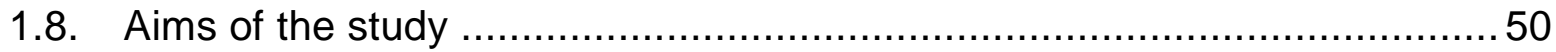

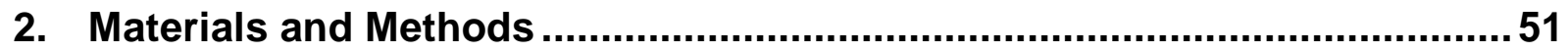

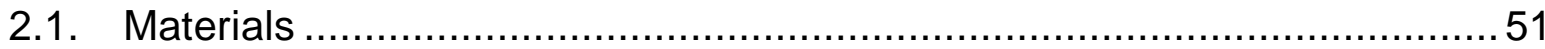

2.1.1 Reagents and consumables ................................................... 51

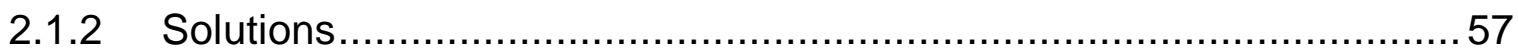

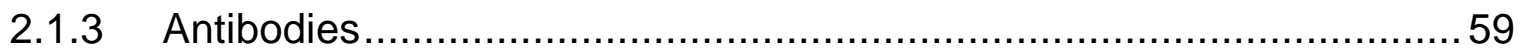

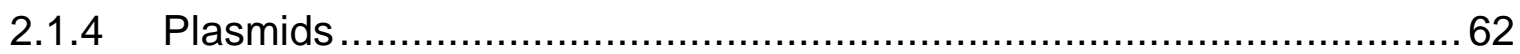

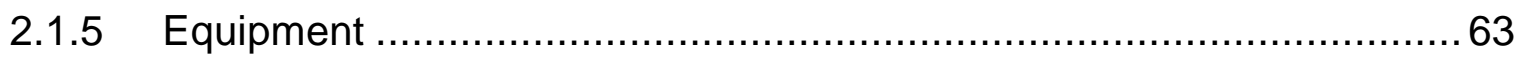

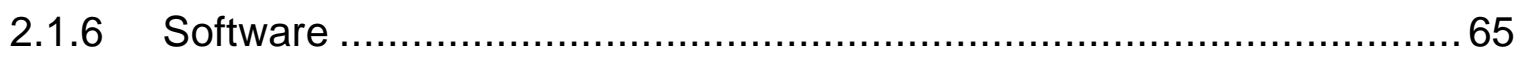

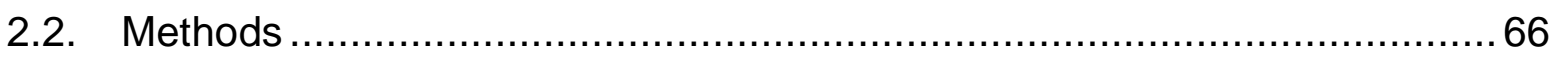

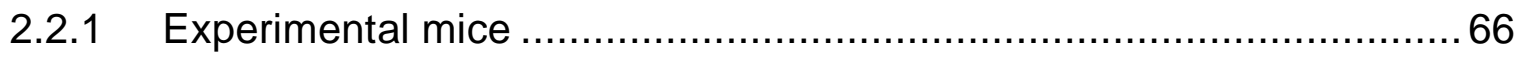

2.2.2 DNA extraction and genotyping.................................................. 66

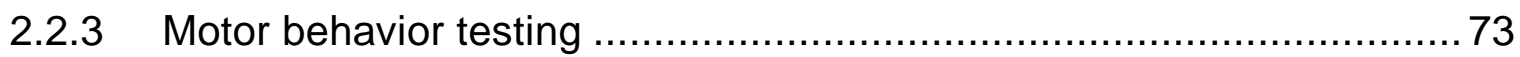

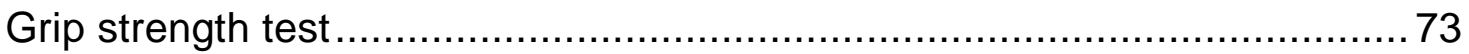

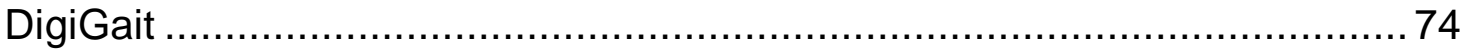

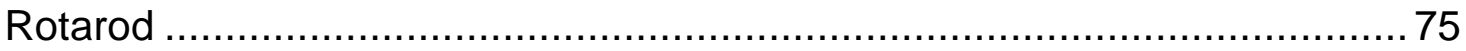




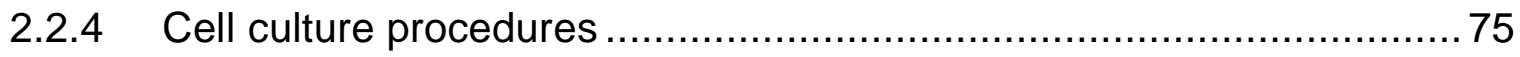

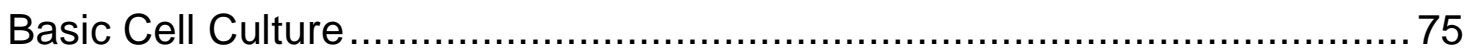

Induction of triple knock-out in dynamin cTKO MEFs ............................... 77

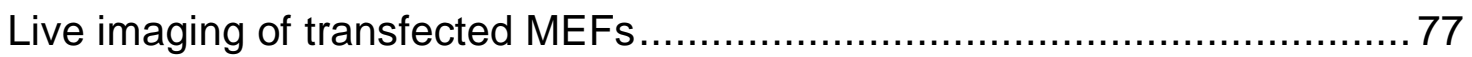

2.2.5 Brain dissection from young and adult mice ................................. 79

2.2.6 Staining procedures on brain slices and cells ................................ 80

Preparation of brain slices for immunostainings .................................... 80

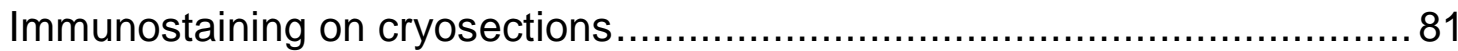

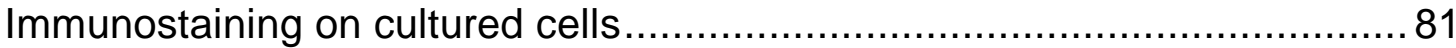

TdT-mediated dUTP-biotin nick end labeling (TUNEL) assay ....................... 81

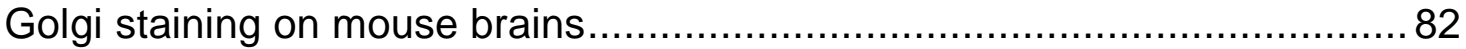

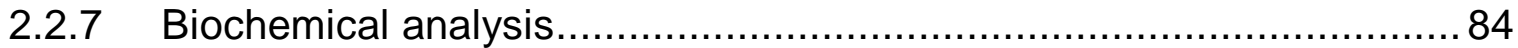

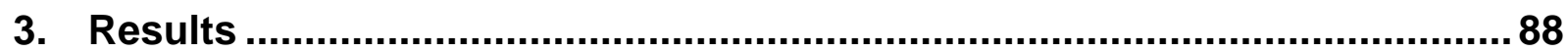

3.1. Characterization of motor behavior tests for aging mice …...................... 88

3.2. Motor behavior in endophilin-A mutants ............................................. 91

3.2.1 Endophilin mutants display reduced muscle strength ........................ 91

3.2.2 Motor coordination is severely impaired in endophilin mutants ............92

3.2.3 Gait rhythm and posture are altered in endophilin mutants ............... 94

3.3. Motor behavior in other endocytic mutants .........................................97

3.3.1 Synaptojanin-1 HT mice have no obvious motor impairments .............97

3.3.2 Dynamin mutants display mild motor impairments ...........................98

3.4. Cell death in endophilin mutants .................................................... 100

3.4.1 TUNEL staining reveals cell death in endophilin DKOs and aged ............ 1KO-2HT-3KO mice but not TKOs ............................................ 100

3.4.2 The apoptosis marker caspase 3 is enriched in endophilin but not in synaptojanin-1 brain sections ........................................... 104

3.4.3 A-synuclein levels and distribution are altered in endophilin DKOs ... 105 
3.5. Autophagy impairments in endophilin mutants .................................. 109

3.5.1 Fbxo32 is misregulated in aged endophilin 1KO-2HT-3KO mice ....... 109

3.5.2 Poly-ubiquitinated proteins accumulate in endophilin mutants but not in synaptojanin-1 or dynamin deficient brains ............................111

3.5.3 Autophagy markers are dysregulated in endophilin mutants ............113

3.6. Rescue of motor impairments in endophilin mutants by genetic reduction of Fbxo32

3.6.1 Fbxo32 co-localizes with endophilin on SV necks

3.6.2 Genetic reduction of Fbxo32 does not affect grip strength in endophilin mutants

3.6.3 Rescue of motor coordination deficits in endophilin mutants by genetic reduction of $\mathrm{Fbxo32}$

3.6.4 Genetic reduction of Fbxo32 has no additional effect on gait rhythm in endophilin mutants

3.6.5 Posture is ameliorated in endophilin mutants after genetic reduction of Fbxo32

3.6.6 Comparison of motor impairments in endophilin mutants with and without genetic reduction of Fbxo32

4. Discussion

4.1. Motor impairments in endocytic mutants

4.2. Neurodegeneration in the endophilin mouse model

4.3. Altered autophagy in endocytic mutants

4.4. Partial rescue of endophilin phenotype by Fbxo32 reduction

4.6. Endophilin's role in synaptic proteostasis - A model

5. Summary and Conclusion

5.1. Perspectives 
Appendix

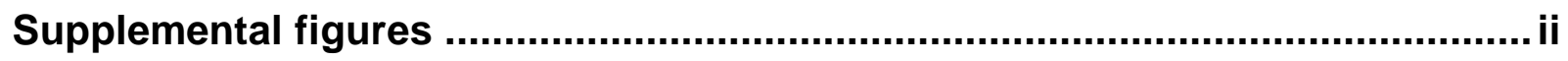

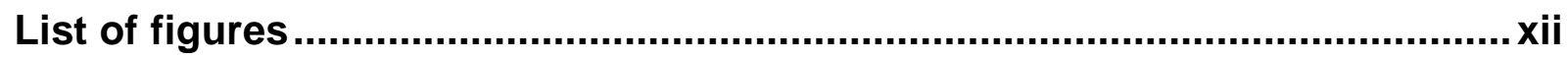

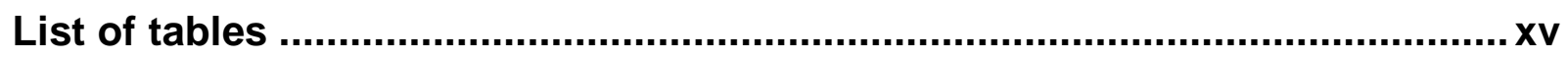

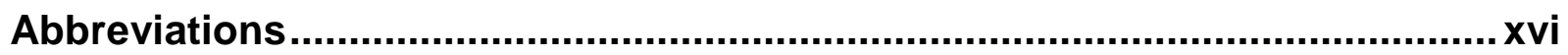

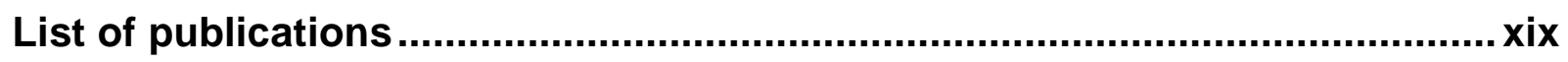

First and co-first author publications ........................................................

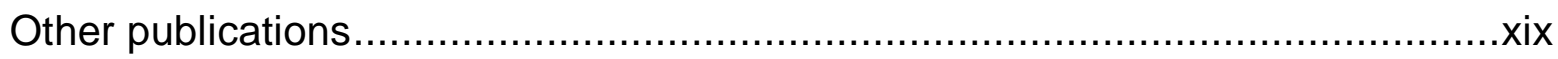

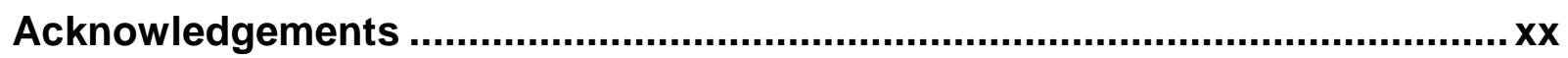

Curriculum vitae ........................................................................................... Xxii 


\section{Abstract}

Neurodegenerative diseases have become an increasing problem in our aging society. Many studies have addressed these complex diseases in the recent years. Scientists have learned a lot about specific proteins, their regulation and roles in diseases, yet there are still many unanswered questions in this area. As the knowledge increases, it has become apparent that these diseases affect more than one protein and that entire processes are dysregulated or dysfunctional, leading to similar but often non-identical symptoms in patients of different neurodegenerative diseases. Emerging evidence is accumulating that while the proteins involved in these diseases are diverse, many of them ultimately disrupt basic cellular processes in neurons. It is therefore not surprising that recent reports have shown that impairments of endocytosis or autophagy, two processes involved in maintaining proteostasis in synapses, can lead to neurodegeneration.

This thesis was designed to shed light on questions of if and how the family of endophilin-As, key proteins in synaptic endocytosis, is involved in neurodegeneration. Taking advantage of the complex endophilin mouse model, this thesis shows that endophilin mutants have progressive motor impairments depending on the number of deleted endophilin alleles and the animal age. The motor deficits are prominently detected in the Rotarod test which requires motor coordination and in the overall running ability on a motorized treadmill and subtly found in gait analysis or general muscle strength.

Furthermore, data presented here also reveal signs of neurodegeneration in mouse models for endophilin deficiency. The evidence points to a mild form of neurodegeneration that is found in the hippocampus, cortex and cerebellum. These findings include an increase in apoptotic and necrotic cells in brain sections from different endophilin mutants and a strong gliosis in endophilin 1,2-DKO mice.

Unexpectedly, data reported in this thesis, complemented by additional data produced in the laboratory, indicate that endophilin has a previously unknown role in autophagy in addition to its well-described role in endocytosis. Most prominently, an E3 ubiquitin ligase, Fbxo32, is robustly up-regulated in various endophilin mutants. 
Fbxo32 has previously been suggested to act as a mediator between the ubiquitinproteasome system and autophagy. Congruously, endophilin mutants are overloaded with poly-ubiquitinated proteins that are marked for degradation through the UPS and have decreased levels of Atg5, a protein involved in the formation of the autophagophore. However, because of this double role of endophilin, it is challenging to discriminate between the impact of endocytosis and autophagy impairments on the systemic level.

In order to answer this question, two additional mouse models for defective endocytosis, synaptojanin-1 and dynamin 1,3 deficient mice, were also tested for motor impairments and the regulation of autophagy markers. In this study, no motor impairments were discovered in aged synaptojanin-1 deficient mice and only mild motor deficits in dynamin mutants. In addition, the amount of poly-ubiquitinated proteins and Atg5 were unchanged in synaptojanin and dynamin deficient mice.

To elucidate how defective autophagy and endocytosis impact motor behavior in the endophilin mouse model, this thesis attempted to normalize altered proteostasis in the endophilin mutants by genetically reducing the levels of Fbxo32. In fact, motor deficits were ameliorated in endophilin mutants who are also heterozygous for Fbxo32. Taken together, this work sheds light on endophilin's role in endocytosis and autophagy and the increasing importance of protein homeostasis on neuronal survival. 


\section{Introduction}

\subsection{The central nervous system and neurodegenerative diseases}

The central nervous system (CNS) consists of the brain and the spinal cord and acts as the control unit of the body. It is characterized by a complex interplay between different types of cells that orchestrate the flow of information in our brains and enables us to interact with our environment. The CNS integrates information from the body and its surroundings, processes them and triggers an appropriate response. Thus, it regulates basic body functions such as body temperature and breathing but it also makes it possible for us to have abstract thoughts, to understand the world around us and to learn from previous experience. Evolutionary speaking, the continuous development of the CNS in mammals, especially primates, has enabled us to orient ourselves in the world, have complex social interactions with each other and even engage in studies of nature, physics or medicine.

The processing of information needed for such complicated actions is achieved through a combination of chemical and electrical signaling in the CNS with the help of cells that were optimized for this task, neurons. They consist of a cell soma and an axon (Figure 1). Sensory neurons react to outside stimuli, for example tactile stimuli in hair cells or chemical stimuli in the olfactory bulb. These stimuli induce an electrical impulse, an action potential, which starts at the soma and is transferred along the axon until it reaches specialized compartments, the synapses. A synapse is the connection point between two neurons or between a neuron and a sensory or motor cell. Synapses consist of a pre-synaptic side, a cleft and a post-synaptic side. On the pre-synaptic side, the electrical signal triggers voltage-gated channels to open which causes a calcium influx in the synapse and thereby the release of chemical components, the neurotransmitters. These transmitters pass the synaptic cleft and bind to receptors on the post-synaptic side where they stimulate the post-synaptic neuron to repeat the same process or execute a specific task. Through a multiplex 


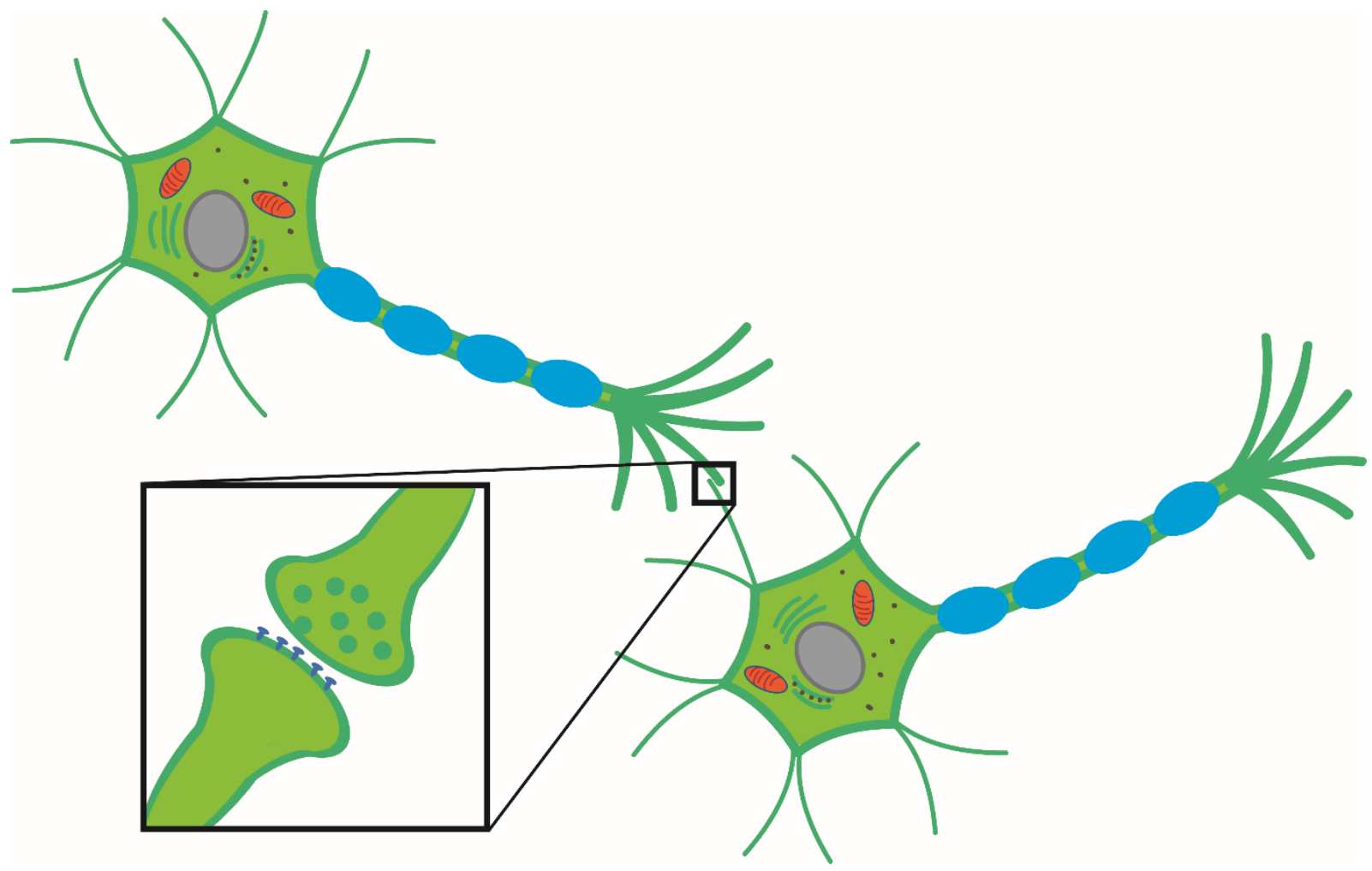

Figure 1 - Schematic representation of neuronal signal transduction. A stimulated neuron carries an electric signal along the insulated axon to the presynaptic terminal. At the synapse (magnified in insert) neurotransmitters are released into the synaptic cleft and detected by receptors on the post-synaptic membrane. Original illustration by C. M. Rostosky.

chaining of neurons, the original stimulus is transported and amplified through the nervous system until it triggers a response, for example a muscle contraction.

The second major type of cells in the CNS are glia cells. While glia cells do not transmit nervous signals, they perform essential tasks by supporting neurons and acting as immune cells inside the brain. For example, astrocytes provide the neurons with nutrients and oxygen and they clear excess neurotransmitters form the synaptic cleft while microglia remove dead neurons from the CNS (reviewed in Khakh \& Sofroniew, 2015; Nayak, Roth, \& McGavern, 2014). In addition, oligodendrocytes provide the electrical insulation of the axons that is crucial to carrying the electrical signal from the soma to the synapse. Interestingly, recent studies point to an even more substantial role of glia cells in the development of the CNS and the regulation of neuronal survival (reviewed in Zuchero \& Barres, 2015). 
Neurons differ in shape and the use of different neurotransmitters and receptors, which has a great influence on the functional output. For example, the released neurotransmitters can excite or inhibit the post-synaptic neuron and thereby increase or decrease the probability that the post-synaptic neuron will produce an action potential. This happens through the use of excitatory neurotransmitters (e.g. glutamate) or inhibitory neurotransmitters (e.g. gamma-aminobutyric acid (GABA), glycine). However, many neurotransmitters can be inhibitory or excitatory depending on the context and especially on the receptors present on the post-synaptic side (e.g. acetylcholine, dopamine).

A neuron usually gets input from several surrounding neurons, both inhibitory and excitatory. In the soma, all inputs are integrated; only when the excitatory signal reaches a specific threshold, does the neuron fire an electrical signal. In mammals, the CNS has developed to form brain regions that function specifically to perform given tasks. For example, the cerebellum is the key region to control motor coordination, neurons in the hippocampus perform learning tasks and the cortex is needed for abstract thinking. The regions are constituted of different types of neurons and glia cells that are organized in a structure supportive to the function. The communication between single neurons in every region as well as between the regions has to be well orchestrated in order to facilitate complex brain function.

If there are problems with this orchestration, it often causes a neurodegenerative disease. These diseases are defined by cell death in the CNS, primarily of neurons with tremendous consequences for the affected patient. The causes for neurodegenerative diseases are often genetic mutations, but also drug abuse or exposure to environmental toxins. Even though the cause, affected brain regions and symptoms are various among several described conditions, one common denominator is that the diseases develop and progress with aging. This becomes especially obvious in the occurrence of dementia, a characteristic of many neurodegenerative diseases. According to the annual health report from the German government 1,640 people of the age of 55-65 years were diagnosed with dementia in German hospitals in 2016 while at the same time 19,874 of the age $75-85$ were diagnosed with the same condition (German health report, 
"Gesundheitsberichterstattung des Bundes"; http://www.gbe-bund.de). The life expectancy increases every year and with that the percentage of older citizens in our society, so we may expect the prevalence of dementia to rise even further in the next years.

As a result, the number of studies about neurodegeneration published each year has doubled from 2007 (2,195 Pubmed entries) to 2017 (4,445 Pubmed entries) and much progress has been made in understanding the specific features of neurodegenerative diseases. One interesting emerging hypothesis from these studies is that problems in homeostasis and protein turnover could be a common cause, or at least act as a contributing factor, for several neurodegenerative diseases (Douglas \& Dillin, 2010; Nixon, 2013; Liang \& Sigrist, 2018). In order to maintain functional proteins, that are necessary to fulfill cellular tasks like synaptic transmission, proteins constantly are degraded and newly transcribed, translated, assembled and transported to necessary location. This process is called proteostasis and is difficult to maintain in synaptic structures because they are often located very far away from the cell soma and the nucleus, where messenger ribonucleic acid (mRNA) is transcribed that contains the information for the protein translation and assembly. According to the hypothesis, the long distance to the cell soma makes synapses the most vulnerable structures in the neuron that will therefore be the first place where defective proteostasis causes negative effects. Since the whole proteostasis machinery becomes less effective with aging (reviewed in Cuervo, et al., 2005; Martinez-Vicente, Sovak, \& Cuervo, 2005), the occurrence of misfolded proteins will cause more and more problems as the system ages. When non-functional proteins accumulate in the synapses, they interfere with the effectiveness of the synaptic signal transduction. Once synapses become non-functional, they are degenerated causing the axons to retract and ultimately neurons to die (Burke \& O'Malley, 2013).

\subsubsection{Synaptic morphology}

Signal transduction at the synapse occurs in specialized compartments on both sides of the synaptic cleft, the pre- and post-synapse. These structures ensure that the 
signal is processed in a precise and fast manner and ultimately causes an appropriate response to the input information from the environment.

The pre-synapse is a small sub-compartment that buds from the neuronal axon. It is filled with small membranous structures, the synaptic vesicles (SVs) which hold the neurotransmitters (Figure 2). When an action potential reaches the pre-synapse, some SVs fuse with the plasma membrane and release the neurotransmitters into the synaptic cleft. As a result, they chemically transmit the signal to the post-synapse, where they are recognized by specific receptors. For the release of the

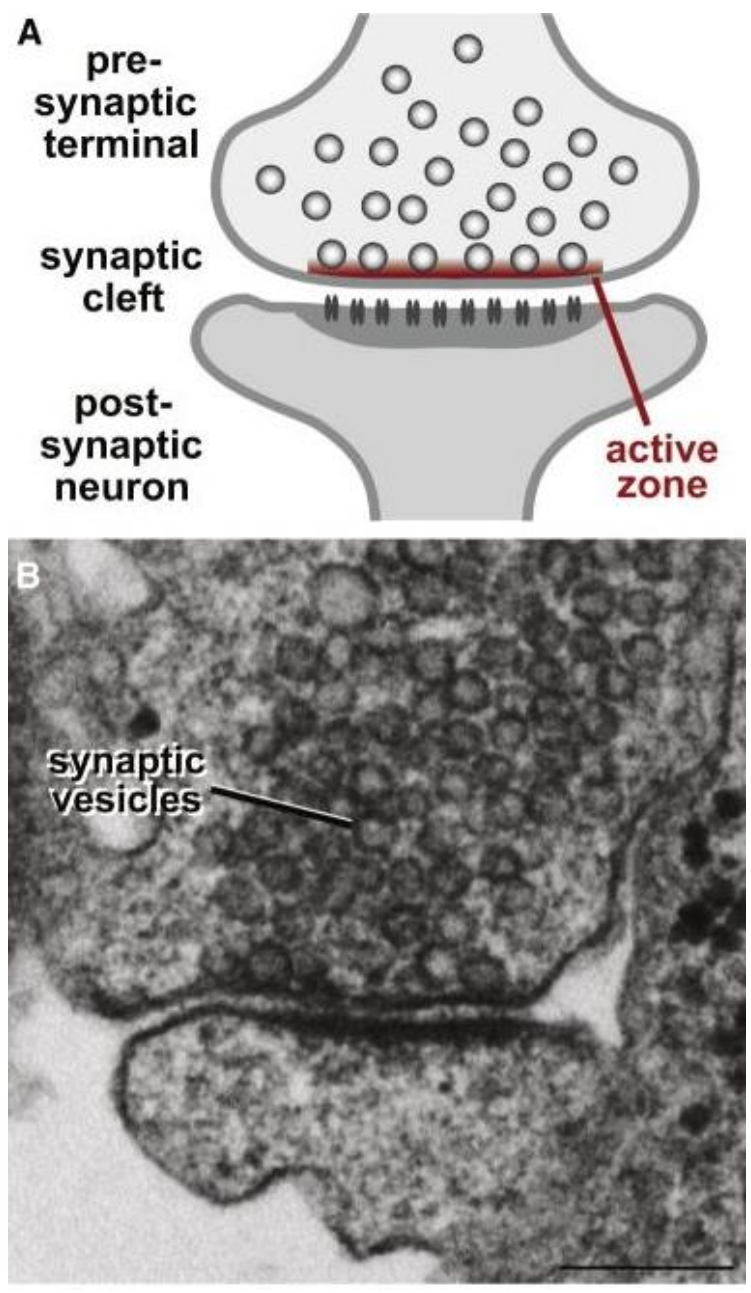

Figure 2 - Structure of a synapse. The pre-synaptic side is characterized by the synaptic vesicles and the electron dense active zone, the post-synaptic side comprises a post-synaptic density which is also electron dense. (A) Schematic representation of a synapse. (B) Electron microscopy image of stained synapse from a hippocampal neuronal culture. Scale bar $200 \mathrm{~nm}$. Modified from (Südhof, 2012) and (Kaeser, et al., 2011). 
neurotransmitters, SVs have to fuse with the plasma membrane in a process called exocytosis. This process consists of three main steps: docking, priming and fusion of the SVs to the plasma membrane. A number of proteins are involved in these steps. They are enriched in the active zone at the plasma membrane. Exocytosis occurs primarily at these active zones (reviewed in Südhof, 2012). The complementary process to exocytosis, which retrieves the excess membrane from the plasma membrane and forms new SVs, is called endocytosis. This process is explained in detail in chapter 1.2 .

The post-synaptic side is located in dendritic spines of a connected neuron. The spines host the post-synaptic density (PSD), the counterpart to the active zone in the pre-synapse. Its defining components are receptors for the neurotransmitters. Upon binding of the neurotransmitter to the receptors, the dendritic spine depolarizes and calcium enters the spine. If enough spines are depolarized in the post-synaptic neuron to exceed the depolarization threshold, the neuron fires an action potential (reviewed in Voglis \& Tavernarakis, 2006).
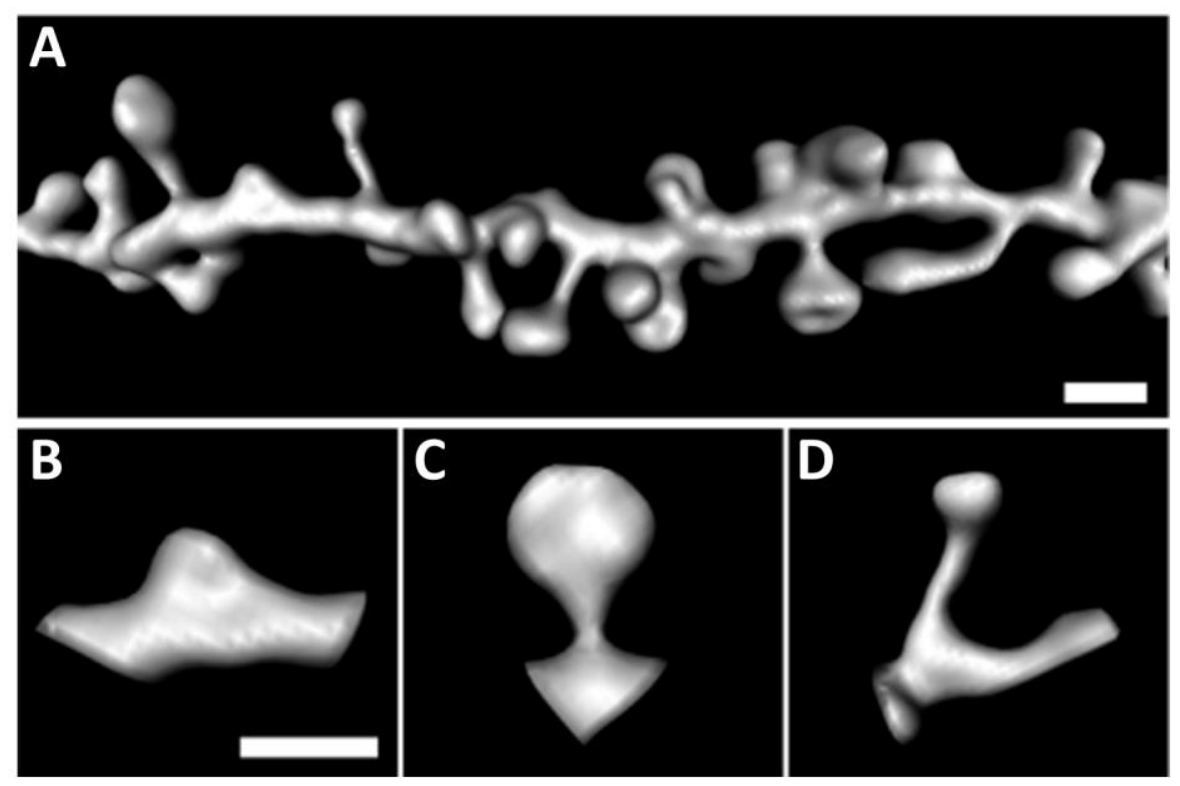

Figure 3 - 3D reconstruction of dendritic spines. (A) Dendrite from a GFPexpressing hippocampal CA1 cell shows several dendritic spines that differ in size and morphology. (B) Example of a stubby spine, defined by a broad and short neck. (C) Example of a mushroom spine, defined by the thin neck and the big, mushroomshaped head. (D) Example of a thin spine, defined by an elongated neck and a thin head. Scale $1 \mu \mathrm{m}$, illustration from (McKinney, 2010). 
A dendritic spine consists of a neck and a head (Hotulainen \& Hoogenraad, 2010). They are morphologically classified into three types of spines: mushroom, stubby and thin (McKinney, 2010; see Figure 3). Importantly, the morphology of dendritic spines is tightly linked to the synaptic strength and is thus an important aspect for signal transduction. The development of high-resolution live-imaging techniques has made it possible to monitor the morphology of synaptic spines during long-term potentiation (LTP) and long-term depression (LTD). The continuous stimulation of spines by LTP leads to an increase in dendritic spine head size while LTD has opposite effects (Matsuzaki, Honkura, Ellis-Davies, \& Kasai, 2004). LTP also causes the generation of new dendritic spines (Engert \& Bonhoeffer, 1999; Fukazawa, et al., 2003; Alger \& Teyler, 1976; Fortin, et al., 2010). Together, these studies show that spine density and morphology are highly plastic and seem to have functional relevance for the synapse (reviewed in Rochefort \& Konnerth, 2012).

The length and head size of the spines are regulated by actin filaments and actin regulators. Head enlargement upon LTP stimulation is mediated by the Arp2/3 complex that causes actin branching (Hotulainen, et al., 2009). The increased size is important to create space for more receptors which then stabilize and strengthen the synapse. Head size also correlates with the number of SVs in the pre-synapse (Harris \& Stevens, 1988; Harris \& Stevens, 1989; Schikorski \& Stevens, 1997). Because of that, mushroom spines, which have a large head, are regarded as mature spines while thin and stubby spines are classifies as young or dormant spines (Matsuzaki, et al., 2001; reviewed in Woolfrey \& Srivastava, 2016). 


\subsection{Endocytosis - an essential process for maintaining synaptic activity}

In order to ensure continuous firing of neurons, the pre-synaptic compartment always needs to have enough SVs to release when an action potential reaches the synapse. The complementary process that is responsible for the replenishment of the SV pool is called endocytosis. It recycles the excess membrane of the fused SV from the plasma membrane to form new SVs that will subsequently be used for a new round of exocytosis. Several mechanisms how endocytosis could work have been hypothesized and observed in studies. These modes of endocytosis include clathrinmediated endocytosis (CME), ultrafast endocytosis, bulk endocytosis and kiss-andrun and are explained in the following sections.

\subsubsection{Clathrin-mediated endocytosis}

The most studied and generally accepted mechanism of endocytosis in the CNS synapse is the clathrin-mediated endocytosis (reviewed in McPherson, Ritter, \& Wendland, 2000-2013). The key factor for this process is the clathrin triskelion that is composed of three clathrin-heavy-chains and 3 clathrin-light-chains. The assembly of several clathrin triskelia creates a football-like structure that encapsulates membrane structures to form SVs (compare Figure 4 and Figure 5A). Clathrin binds to adapter proteins, most prominently to adaptor protein 2 (AP2) that is recruited to the plasma membrane by a specific phospholipid, phosphatidylinositol $(4,5)$ bisphosphate $\left(\mathrm{PI}(4,5) \mathrm{P}_{2}\right)$. AP2 is also responsible to recruit transmembrane proteins (cargo) to the SVs: it binds to $\mathrm{PI}(4,5) \mathrm{P}_{2}$, cargo and one arm of a clathrin triskelion, thereby initiating the formation of the clathrin coat.

Upon assembly of several clathrin triskelia, the membrane starts to form clathrincoated pits (CCPs). The curvature of the neck of the CCP, that connects it to the plasma membrane, is recognized by the BIN1/amphiphysin/Rvs167p (BAR) domain of endophilin-A. Endophilin-A binds to the neck of the CCP and further enhances the membrane curvature. Endophilin-A also has a SRC Homology 3 (SH3) domain that 
recruits other factors to the clathrin-coated structures that are involved in endocytosis (Cestra, et al., 1999; Verstreken, et al., 2003; Sundborger \& Hinshaw, 2014).

One such protein is the dynamin GTPase that is responsible for the fission of the SV from the membrane (Sundborger \& Hinshaw, 2014). However, dynamin also localizes to CCPs in the absence of endophilin, showing that the recruitment can be compensated by other proteins (Milosevic, et al., 2011). It binds to the $\mathrm{PI}(4,5) \mathrm{P}_{2}$ on the neck of the CCPs through its pleckstrin homology $(\mathrm{PH})$ domain and uses the energy set free by GTP hydrolysis to provide a twisting force that cuts the neck.

Another protein that is recruited by endophilin-A is synaptojanin-1 (Verstreken, et al., 2003). Synaptojanin-1 is a polyphosphoinositide phosphatase (McPherson, et al., 1996) that dephosphorylates $\mathrm{PI}(4,5) \mathrm{P}_{2}$ and thus reduces the binding affinity of the adaptor proteins. The change of the lipid composition of the clathrin-coated vesicle (CCV) recruits the uncoating factor heat shock cognate 70 (Hsc70). This ATPase together with another cofactor, auxilin, releases the clathrin coat using the energy from ATP hydrolysis.

In the final step of the cycle, the SVs need to mature to be functional for a new cycle of exocytosis. The SVs are re-filled with neurotransmitters via the cargo in the membrane and acidified by the vATPase (Rizzoli, 2014).

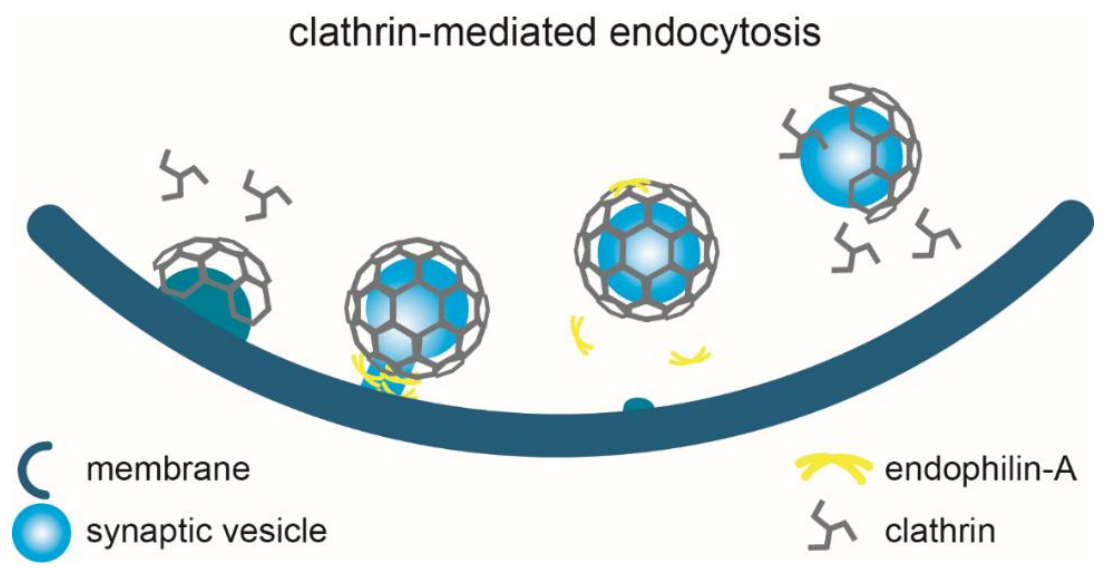

Figure 4 - Clathrin mediated endocytosis. Clathrin is recruited to the plasma membrane and helps to form SVs. The neck of the vesicles is covered with endophilin molecules that tighten the neck for the fission through dynamin 1. The newly formed SV sheds the clathrin coat and matures to become a functional SV for a new round of exocytosis. Original illustration by C. M. Rostosky. 


\subsubsection{Ultrafast endocytosis}

The time estimates for CME are several seconds to $1 \mathrm{~min}$ and it can be argued that this time frame would not be sufficient to control responses to stressful situations like a flight-or-fight decision. Therefore, Watanabe et al. proposed a mechanism for endocytosis that requires 50-100 ms (Watanabe, et al., 2013). In their study, they stimulate hippocampal neurons with a single optogenetic stimulus, freeze the neurons rapidly with high pressure and study synaptic terminals by electron microscopy. They observed large membrane structures lateral to the fusion site that are not SVs and have no clathrin coat, pointing to a clathrin independent mode of endocytosis (compare Figure 5D).

In a follow-up study, Watanabe et al. report evidence that the large membrane structure fuses with an endosome, forming a synaptic endosome that is subsequently resolved into clathrin-coated vesicles (Watanabe, et al., 2014; reviewed in Watanabe \& Boucrot, 2017). Interestingly, the authors later found that endophilin and synaptojanin-1 play a role in this process (Watanabe, et al., 2018). The authors support data that the sorting of the synaptic endosome into SVs happens with the same mechanism as CME and within a time frame of 5-6 s. This finding would point to a primary role of clathrin in sorting membrane into several SVs independent on where the membrane originates (i.e. plasma membrane of synaptic endosome). The goal of clathrin-dependent vesicle formation could be to produce SVs that have the same characteristics (size, cargo) enabling the same output for every action potential that reaches the synapse. However, it remains an open question if ultrafast endocytosis is a general mechanism, a mechanism occurring only in a specific cell type or if its occurrence depends on the synaptic activity, e.g. happens only in synapses with a high turnover.

\subsubsection{Activity-dependent bulk endocytosis}

Activity-dependent bulk endocytosis (ADBE) was first described in 1984 as large plasma membrane invaginations following a strong stimulation (Miller \& Heuser, 1984). Since then, this mode of endocytosis has been studied further, showing that indeed ADBE occurs only after strong stimulation, most likely in cases where CME is 
not able to retrieve SVs from the plasma membrane before the next round of exocytosis (reviewed in Clayton \& Cousin, 2009). Given its activity-dependence, Wu et al., could successfully show that its occurrence is controlled by the calcium sensor calmodulin (Wu, et al., 2009).

In ADBE, the excess membrane from the fusion of many SVs is quickly retrieved in a very large, endosome-like structure (bulk endosome) from which smaller SVs are later sorted (compare Figure 5C). The invagination of the plasma membrane is likely actindriven and its fission from the plasma membrane is dependent on dynamin. Similar to ultrafast endocytosis, the final formation of the SVs is also dependent on clathrin (Takei, Mundigl, Daniell, \& De Camilli, 1996; Gad, Löw, Zotova, Brodin, \& Shupliakov, 1998; Andersson, Jakobsson, Löw, Shupliakov, \& Brodin, 2008).

While the ADBE has a mechanism similar to ultrafast endocytosis, there are some important differences: ultrafast endocytosis happens much faster than ADBE and the amount of internalized membrane is usually larger in ADBE. Moreover, while the endocytosed membrane in ultrafast endocytosis fuses with an endosome for sorting, the bulk endosome directly serves as the sorting station in ADBE (Watanabe \& Boucrot, 2017).

\subsubsection{Kiss-and-run endocytosis}

Kiss-and-run endocytosis was hypothesized as a fast and efficient coupling of exoand endocytosis. In this model, the SV does not fully fuse with the plasma membrane during exocytosis, but forms only a pore to the synaptic cleft. Through this pore, the neurotransmitters leave the SV which subsequently is pinched off again from the plasma membrane as an already complete SV that only needs to re-acidify and be filled with new neurotransmitters (compare Figure 5B).

The existence of this process is however subject of much debate. Because of the fast time frame and the fact that the process is hard to distinguish from the early steps of fusion during exocytosis, it is difficult to prove or disprove kiss-and-run endocytosis (Rizzoli \& Jahn, 2007). One of the strongest points in favor of the kiss-and-run hypothesis is the observation that endocytosis can occur faster than the time frame 
of CME allows. However this could also be achieved through ultrafast or bulk endocytosis (see chapters 1.2.2 and 1.2.3) or through the existence of a "readilyretrievable pool", an assembly of mature SVs that can quickly be exocytosed after stimulation. Evidence for such a pool was presented by two groups in 2006 (Farnández-Alfonso, Kwan, \& Ryan, 2006; Wienisch \& Klingauf, 2006).

Other studies have used various forms of labeled SVs and analyzed uptake, release or quenching of these labels over time. One prominent study used hydrophobic (FM 1-84 and FM 1-43) and hydrophilic styryl dyes (FM 2-10) to label SVs. The study reports that upon fusion with the plasma membrane, the hydrophilic dye was released faster from the SVs than the hydrophobic dyes. The author argued that the release

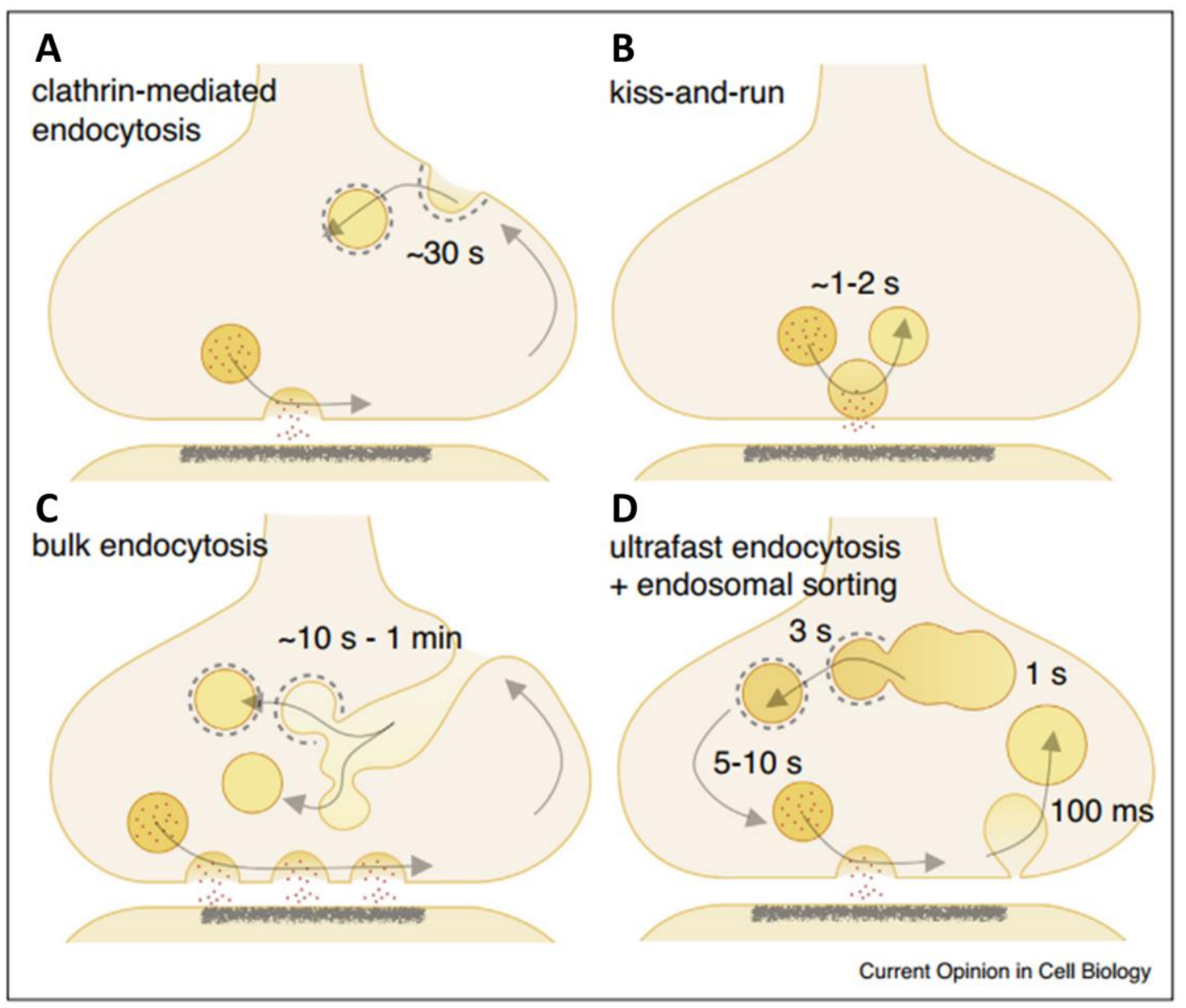

Figure 5 - Modes of endocytosis. Graphic representation of $(A)$ clathrin-mediated endocytosis, (B) kiss-and-run, (C) bulk endocytosis and (D) ultrafast endocytosis. Illustration from (Watanabe \& Boucrot, 2017). 
speed would be the same for both dyes if the SV would completely collapse into the plasma membrane (Klingauf, Kavalali, \& Tsien, 1998). This observation was later challenged by two studies that used similar approaches but could not reproduce the finding (Richards, Guatimosim, \& Betz, 2000; Fernández-Alfonso \& Ryan, 2004; reviewed in Rizzoli \& Jahn, 2007).

Whether or not kiss-and-run endocytosis exists will remain controversial and can probably only be decided by the development of new tools that allow an even better spatial and temporal resolution. 


\subsection{Key proteins in endocytosis}

\subsubsection{Endophilin-A}

In vertebrates, the family of endophilin proteins consists of three members of the endophilin-A sub-family ( $A 1, A 2$ and $A 3)$ and two members of the endophilin-B subfamily (B1 and B2). Endophilin-A was discovered in 1997, endophilin-B in 2000 (Ringstad, Nemoto, \& De Camilli, 1997; Micheva, Kay, \& McPherson, 1997; Huttner \& Schmidt, 2000; Farsad, et al., 2001; Pierrat, et al., 2001). All family members possess an $\mathrm{N}$-terminal BAR domain and a $\mathrm{C}$-terminal $\mathrm{SH} 3$ domain and form crescentshaped homodimers (Modregger, Schmidt, Ritter, Huttner, \& Plomann, 2003; Gallop, et al., 2006; Masuda, et al., 2006; Weissenhorn, 2005; s. Figure 6). Endophilin-A1 is brain specific, while $A 2$ is ubiquitously expressed and $A 3$ is enriched in brain and testis (Giachino, et al., 1997; Ringstad, Nemoto, \& De Camilli, 1997; So, et al., 2000). Endophilin-B1 and B2 were detected in several tissues including brain, but are not ubiquitously expressed (Farsad, et al., 2001; Modregger, Schmidt, Ritter, Huttner, \& Plomann, 2003).

Endophilin-As are best studied for their role in CME (s. chapter 1.2.1) but have recently also been implied to play a role in clathrin-independent endocytosis, more specifically in ultrafast endocytosis (Boucrot, et al., 2015; Watanabe, et al., 2018). Because of endophilin-As role in endocytosis, it is not surprising that it is concentrated at pre-synaptic terminals (Bai, Hu, Dittman, Pym, \& Kaplan, 2010). Endophilins-B on the other hand are rarely observed on synaptic structures but are rather cytosolic in

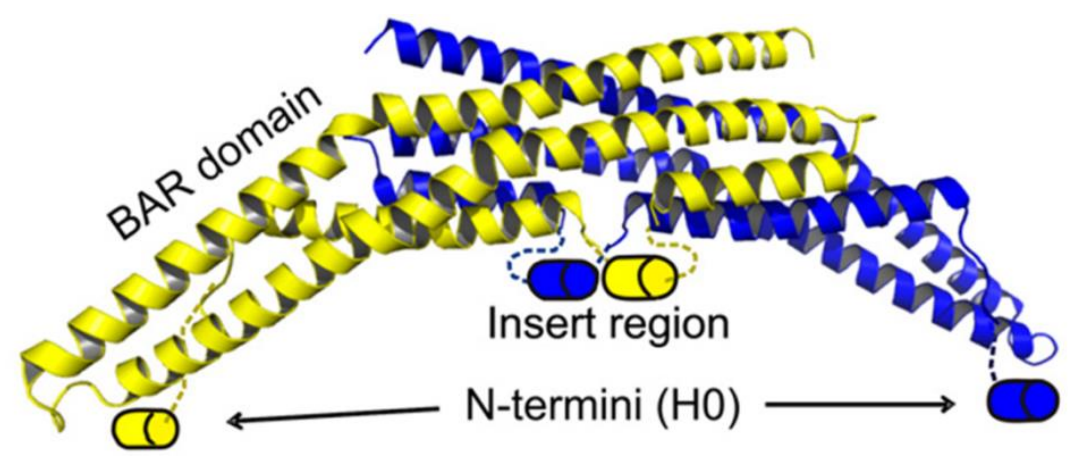

Figure 6 - Crystal structure of an endophilin-A1 dimer. Subunits are colored in blue or yellow. Illustration from (Ambroso, Hegde, \& Langen, 2014). 
the perinuclear region or bound to other intracellular membrane structures (e.g. mitochondria; Farsad, et al., 2001; Modregger, Schmidt, Ritter, Huttner, \& Plomann, 2003). They are hypothesized to play a role in autophagy and apoptosis (reviewed in Kjaerulff, Brodin, \& Jung, 2011).

Endophilin-A has been shown to perform two important tasks during endocytosis: it recruits essential players to the forming CCP, namely dynamin and synaptojanin, through the SH3 domain (Meinecke, et al., 2013; Ringstad, et al., 1999; Verstreken, et al., 2003; Milosevic, et al., 2011). It also induces membrane curvature at the neck of CCPs through the BAR domain (Bai, Hu, Dittman, Pym, \& Kaplan, 2010; Farsad, et al., 2001; Gallop, et al., 2006; Masuda, et al., 2006).

The induction of membrane curvature was originally proposed to be achieved through a scaffolding mechanism in which the crescent shape of endophilin-A dimers causes the membrane to bend in the same way (Blood \& Voth, 2006; Blood, Swenson, \& Voth, 2008; Arkhipov, Yin, \& Schulten, 2009). More recent studies have since shown, that the BAR domain of endophilin on SVs actually resides at a significant distance from the membrane, making it unlikely that endophilin acts only through a scaffolding mechanism (Jao, et al., 2010). The authors instead observed amphipathic $\alpha$-helices that embed into the plasma membrane upon binding and employ membrane curvature induction through a wedging mechanism (compare Figure 6). However, in a follow-up study they report that tubulation depends on the scaffolding mechanism (Ambroso, Hegde, \& Langen, 2014) pointing to different curvature inducing mechanisms on the neck and surface of CCPs. They further propose that phosphorylation of the insertion site by Leucine-rich-repeat kinase 2 (LRRK2), a kinase strongly associated with PD, acts as a regulation mechanism to switch between scaffolding and wedging curvature induction. Further evidence for such a mechanism was recently reported in a drosophila model (Soukup, et al., 2016).

\subsubsection{Synaptojanin-1}

Synaptojanins are members of the inositol-5-phosphatase family which is defined by its property to dephosphorylate phosphoinositide lipids in membranes. They thereby 
contribute to the regulation of membrane phosphorylation status and various processes that rely on different membrane compositions, as for example endocytosis (Pirruccello \& De Camilli, 2013).

There are two synaptojanins, synaptojanin-1 and -2 . Both of them contain an Nterminal Sac1 domain, a central 5-phosphatase domain and a C-terminal proline-rich domain (PRD). The Sac1 domain dephosphorylates the phosphoinositides PI4P, $\mathrm{PI} \mathrm{P}$ and $\mathrm{PI}(3,5) \mathrm{P}_{2}$ and the 5-phosphatase domain dephosphorylates $\mathrm{PI}(4,5) \mathrm{P}_{2}$ and $\mathrm{PI}(3,4,5) \mathrm{P}_{3}$ (Guo, Stolz, Lemrow, \& York, 1999). While the Sac1 and 5-phosphatase domain are similar, the PRD is very different between the two isoforms (Nemoto, Arribas, Haffner, \& De Camilli, 1997). Both synaptojanins are highly expressed in the brain, but synaptojanin-2 is additionally expressed in muscle tissue and the testis (Nemoto, Arribas, Haffner, \& De Camilli, 1997). They also differ in their subcellular localization: while synaptojanin-1 was found to be enriched in synaptic terminals (McPherson, Takei, Schmid, \& De Camilli, 1994), synaptojanin-2 reportedly localizes to the cytoplasm, mitochondria and the plasma membrane (Nemoto \& De Camilli, 1999; Malecz, et al., 2000).

Because of its brain specificity and its localization to synaptic structures, synaptojanin-1 was proposed to act in endocytosis. In fact, synaptojanin-1 is recruited to SVs by its PRD through the endophilin SH3 domain (Cestra, et al., 1999; Verstreken, et al., 2003). At the SVs, it dephosphorylates $\mathrm{PI}(4,5) \mathrm{P}_{2}$ through its 5phosphatase domain (McPherson, et al., 1996; Cremona, et al., 1999; Mani, et al., 2007; Chang-lleto, et al., 2012). This change of the lipid composition reduces the binding affinity of the adapter proteins and enables the recruitment of the uncoating factor heat shock cognate 70 (Hsc70).

Interestingly, synaptojanin-1 was recently found to also have a role in other forms of endocytosis. Like endophilin, it was shown to act in ultrafast endocytosis by facilitating the formation of the neck after membrane invagination by its 5 -phosphatase activity (Watanabe, et al., 2018). 


\subsubsection{Dynamin}

The scission of the SV neck that pinches off the SV from the plasma membrane is achieved by the GTPase dynamin (Sundborger \& Hinshaw, 2014). Dynamin is encoded by three dynamin genes, dynamin 1, 2 and 3. Dynamin 1 is brain-specific while dynamin 2 is expressed ubiquitously and dynamin 3 is expressed in several tissues including the brain but is not ubiquitously expressed (Cao, Garcia, \& McNiven, 1998). In the brain, dynamin 1 is the most enriched of the three proteins and locates to synaptic terminals (McPherson, Takei, Schmid, \& De Camilli, 1994; Cao, Garcia, \& McNiven, 1998; Ferguson, et al., 2007).

Dynamins have four functional domains: an N-terminal GTPase domain, a pleckstrin homology domain, a GTPase effector domain and a C-terminal PRD (reviewed in Hinshaw, 2000). The PRD contains several SH3 binding sites that are for example recognized by endophilin or amphiphysin and has therefore been proposed to be recruited through this domain (Grabs, et al., 1997; Ringstad, Nemoto, \& De Camilli, 1997). However, dynamin is recruited to endocytic pits even in the absence of endophilin (Milosevic, et al., 2011).

Dynamins form cytosolic tetramers that then oligomerize on the necks of the SVs. The binding to the neck is facilitated through the pleckstrin homology domain that binds the $\mathrm{PI}(4,5) \mathrm{P}_{2}$ on the SV neck. The function of dynamin is further enhanced by the GTPase effector domain that activates the GTPase domain. After the oligomerization, this GTPase domain hydrolyses GTP. The energy set free by this reaction is used to create a twisting force that further tabulates the membrane and ultimately cuts it (reviewed in Hinshaw, 2000).

Even though dynamin has been extensively studied for its role in CME, studies show that it also participates in clathrin independent modes of endocytosis (reviewed in Mayor, Parton, \& Donaldson, 2014). 


\subsubsection{Auxilin}

Auxilin is an important cofactor for Hsc70 and both of them are necessary for effective uncoating of CCVs (s. chapter 1.2.1). It is neuron specific and enriched in synaptic terminals (Ahle \& Ungewickell, 1990). Auxilin contains three domains: the N-terminal PTEN-like domain facilitates the binding to $\mathrm{PI}(4,5) \mathrm{P}_{2}$ (Lee, Wu, Eisenberg, \& Greene, 2006; Massol, Boll, Griffin, \& Kirchhausen, 2006), the central region contains binding motifs for AP-2, clathrin and dynamin (Scheele, Kalthoff, \& Ungewickell, 2001; Newmyer, Christensen, \& Sever, 2003) and the C-terminal J domain carries a histidine-proline-asparatic acid motif through which auxilin binds to Hsc70 (Eisenberg \& Greene, 2007).

The cyclin-G-dependent kinase is a homologous protein to auxilin. It is ubiquitously expressed and up-regulated in the brain in the absence of auxilin (Yim, et al., 2010). Therefore, the loss of auxilin can be partly - but not completely - compensated by cyclin-G-dependent kinase. 


\subsection{Mouse models of defective endocytosis}

Mouse models for the most important players in endocytosis have been available for several years. They are an important tool for studying the in vivo relevance of the specific protein. They further allow studying consequences of the deletion on an organismic scale. Given that the process of endocytosis is disrupted in all of these animals, they all share similar phenotypes. In this section, the key aspects of the available mouse lines are summarized:

\subsubsection{Endophilin-A mouse models}

Mouse mutants carrying a triple knock-out (TKO) of all three endophilins-A show that endophilin-A is essential for the survival of the mouse, as complete knock-out (KO) results in death of the animals just a few hours after birth (Milosevic, et al., 2011).
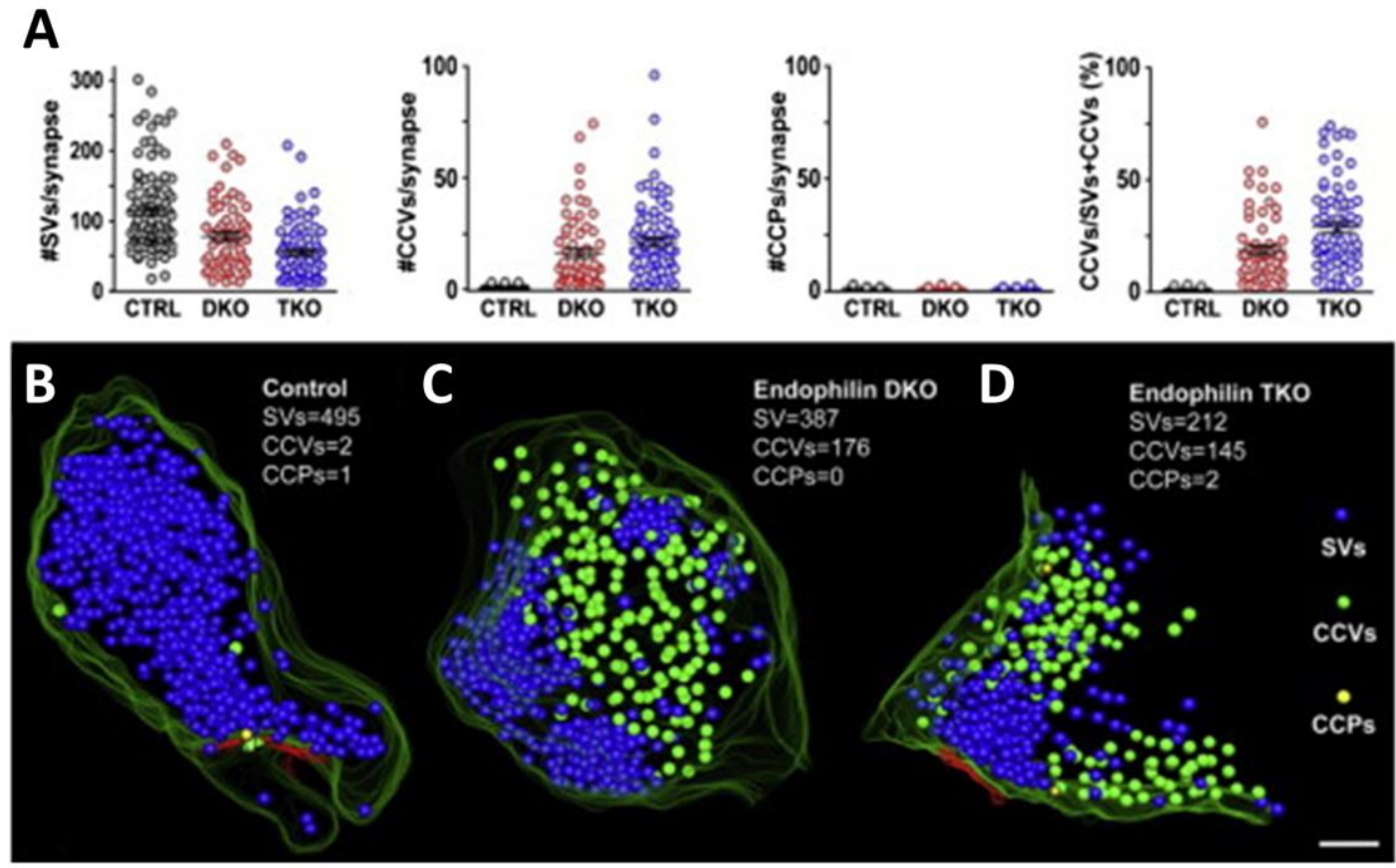

Figure 7 - Accumulation in CCVs in endophilin deficient neurons. (A) Quantification of clathrin coated structures shows that relative number of CCVs is increased in endophilin DKO and TKO neurons. (B-D) Tomographic reconstruction of a series of ultrathin sections from control (B), endophilin DKO (C) and TKO neurons (D). From (Milosevic, et al., 2011). 
However, deletion of endophilin A1 and A2 (DKO) causes a reduction of live span to 14-21 days and ataxia whereas mice that have a KO of endophilin 1 and 3 and are heterozygous (HT) for endophilin 2 (endophilin-1KO-2HT-3KO mice) live up to 18 months and develop epileptic seizures as they age showing that deletion of specific endophilin-As can be at least partially compensated by other endophilins (Milosevic, et al., 2011).

Electron microscopy analysis of synapses from TKO brains revealed an accumulation of CCPs and CCVs, showing that the process of endocytosis is disrupted in these brains at the level of both fission and uncoating of SVs (Figure 7; Milosevic, et al., 2011). Excitation of TKO neurons with sustained stimuli results in increased depression time and the recovery is significantly delayed in these cells (Milosevic, et al., 2011). This indicates that defects in endocytosis have functional consequences for exocytosis and synaptic transmission.

Endophilin TKO and DKO hippocampi were analyzed by next-generation-sequencing (NGS). NGS is a state-of-the-art technique to quantify levels of mRNA in a reliable, fast and cost-efficient manner. The read-out is a list of differentially expressed genes
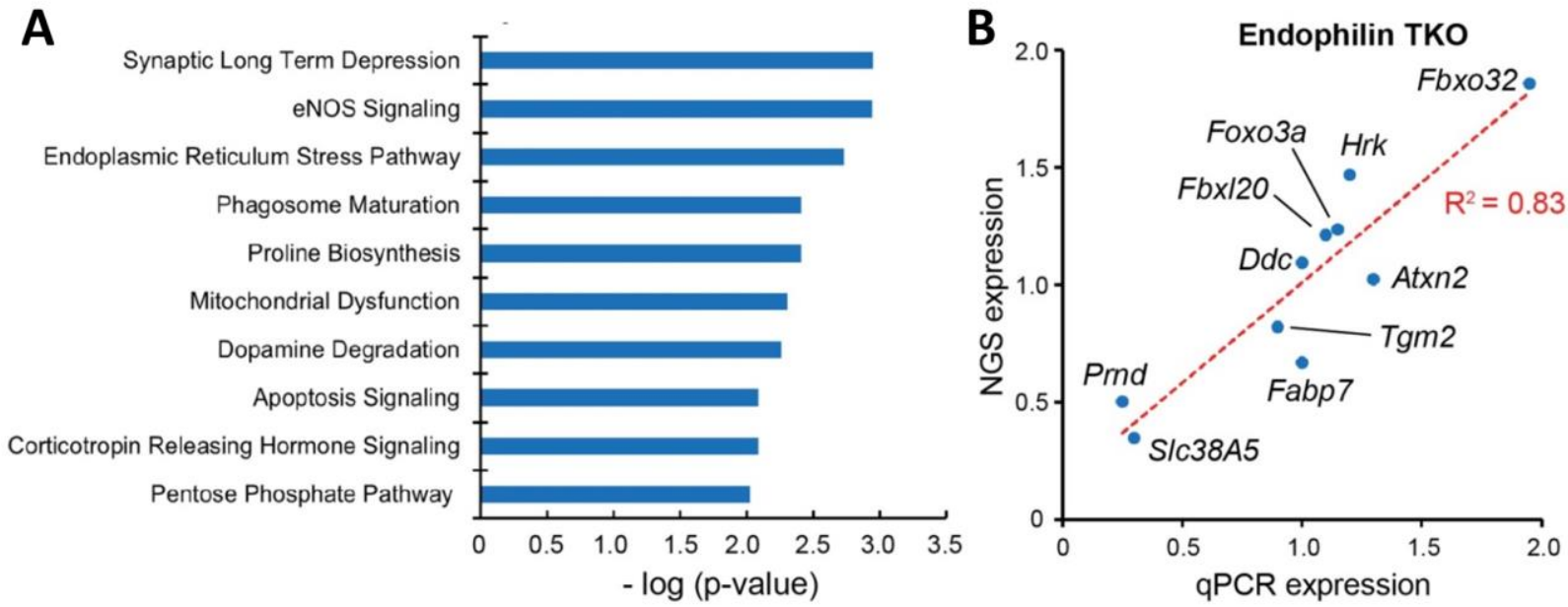

Figure 8 - Pathway analysis and upregulation of Fbxo32 in endophilin TKO brains. (A) Ingenuity pathway analysis of next-generation sequencing (NGS) results of endophilin TKO hippocampi reveals the ten most changed pathways. (B) Correlation of NGS and QPCR levels of up-regulated proteins in endophilin TKO hippocampi. Note that Fbxo32 is highly up-regulated both by NGS and qPCR. From (Murdoch, et al., 2016). 
(verses control, DEGs). Ingenuity pathway analysis revealed that synaptic transmission as well as autophagy and degradation pathways are affected in endophilin TKO animals (Figure 8 and Murdoch, et al., 2016).

Interestingly, when looking at single proteins, the expression that was up-regulated most strikingly, was the E3-ubiquitine ligase F-Box protein 32 (Fbxo32 or atrogin-1, see chapter 1.6). Overexpression was further verified by quantitative PolymeraseChain-Reaction (qPCR, Figure 8 and Murdoch, et al., 2016). This up-regulation and the changed stress response, apoptosis and protein degradation pathways point to a misregulation of protein homeostasis in endophilin mutants which could have possible effects on cell survival as will be further discussed in this thesis.

\subsubsection{Synaptojanin-1 mouse models}

Synaptojanin-1 KO mice were first presented by (Cremona, et al., 1999). These mice carry a disruption in the poly-phosphoinositide phosphatase, synaptojanin-1, a key factor for the uncoating of SVs during CME (s. chapter 1.3.1). KO of synaptojanin-1 causes the animals to die within a few hours after birth. The synaptic terminals in these animals show a high abundance of CCVs, and at the same time a reduction of
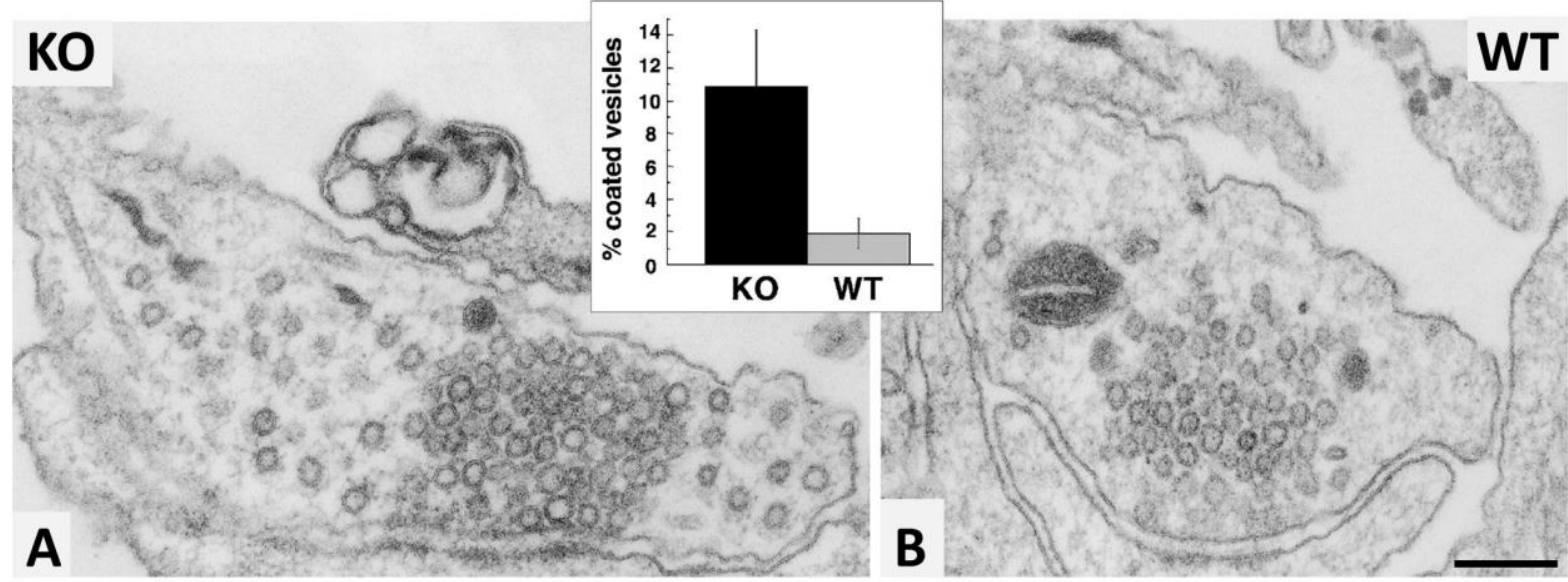

Figure 9 - Accumulation of CCVs in synaptojanin-1 KO synaptic terminals. Electron microscopy images of a KO (A) and WT (B) synaptic terminal in cortical neurons. Scale bar $160 \mathrm{~nm}$. Bar graphs shows percentage of CCVs as mean value \pm SD. Modified from (Cremona, et al., 1999). 
uncoated SVs (Figure 9, and Cremona, et al., 1999). This phenotype results in increased synaptic depression after prolonged stimulation, likely because the fusion pool of SVs is not replenished quickly enough (Cremona, et al., 1999).

Synaptojanin-1 HT mice have a normal life span and do not display any obvious phenotype.

Recently, another synaptojanin-1 mouse model was published that carries a R258Q mutation in the Sac domain (Cao, et al., 2017). This mutation was found in patients with Early-Onset Parkinsonism (Krebs, et al., 2013; Quadri, et al., 2013; Olgiati, et al., 2014). Similar to synaptojanin $\mathrm{KO}$, neurons from these $S J 1^{\mathrm{RQ}}$-knock-in (KI) mice showed an accumulation of CCVs. In addition, the mutation caused structural changes in dopaminergic nerve terminals of the dorsal striatum (Cao, et al., 2017).

These mice display motor deficits, seizures and developmental problems. Only $60 \%$ of them survive until adulthood. However, $\mathrm{HT}$ SJ1 ${ }^{\mathrm{RQ}}-\mathrm{KI}$ mice have a normal life span and no phenotype (Cao, et al., 2017).

\subsubsection{The dynamin-1,3 mouse model}

Two dynamin mouse models have been generated: dynamin $1 \mathrm{KO}$ mice (Ferguson, et al., 2007) and dynamin 1,3 DKO mice (Raimondi, et al., 2011). The dynamin single
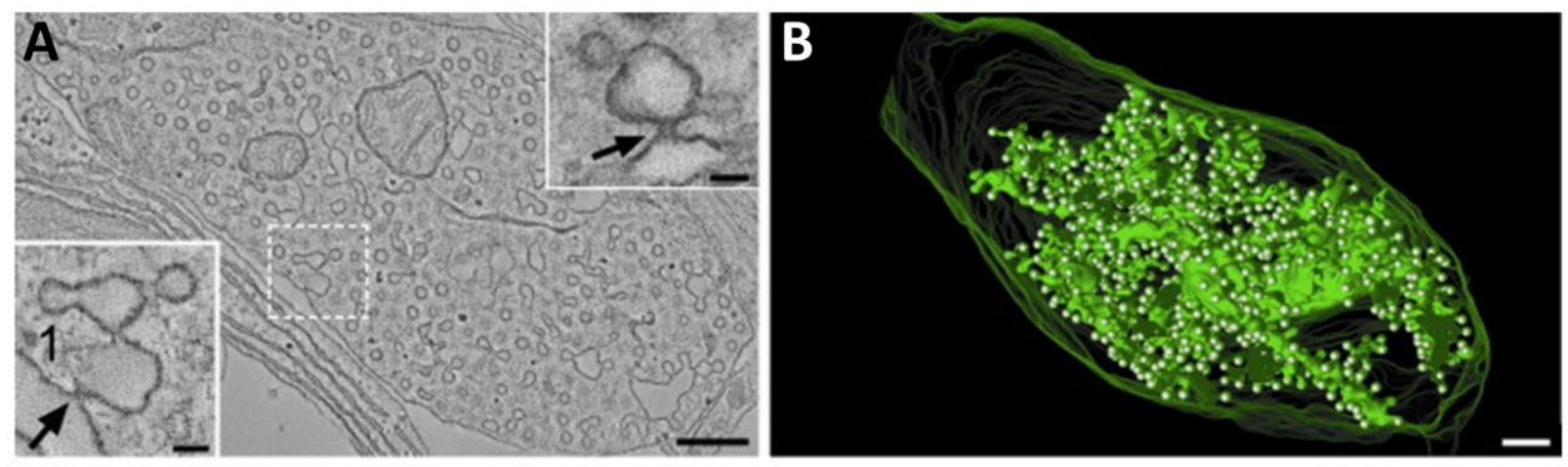

Figure 10 - Accumulation of CCPs in the dynamin 1,3 DKO synapse. (A) electron microscopy image of pre-synaptic terminals shows an accumulation of clathrin coated structures. (B) Tomographic reconstruction of a series of ultrathin sections reveals that the clathrin coated structures are connected to the membrane and are thus CCPs. Scale bar: $250 \mathrm{~nm}$, insets: $50 \mathrm{~nm}$. From (Raimondi, et al., 2011). 
$\mathrm{KO}$ animals die within 2 weeks and accumulate CCPs in synaptic terminals. Basic synaptic transmission is still functional in dynamin $1 \mathrm{KO}$ neurons but the recovery is significantly slowed down which causes defects in synaptic transmission upon sustained stimulation.

Mice missing dynamin 1 and 3 in general have a stronger phenotype than the dynamin $1 \mathrm{KO}$ mice. New-born pups die within only a few hours after birth and the accumulation of CCPs is even more prominent (Figure 10 and Raimondi, et al., 2011). Synaptic transmission is also less efficient in the DKO neurons, however it is not completely abolished (Raimondi, et al., 2011).

\subsubsection{The auxilin mouse model}

The KO of auxilin in mice causes early mortality in the first three weeks, but mice surviving that long then continue to have a normal life span (Yim, et al., 2010). In

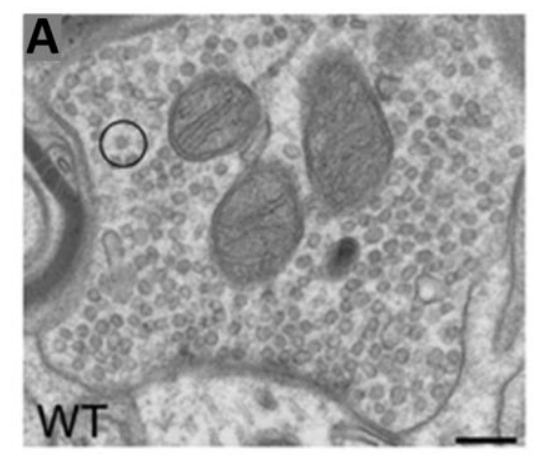

B
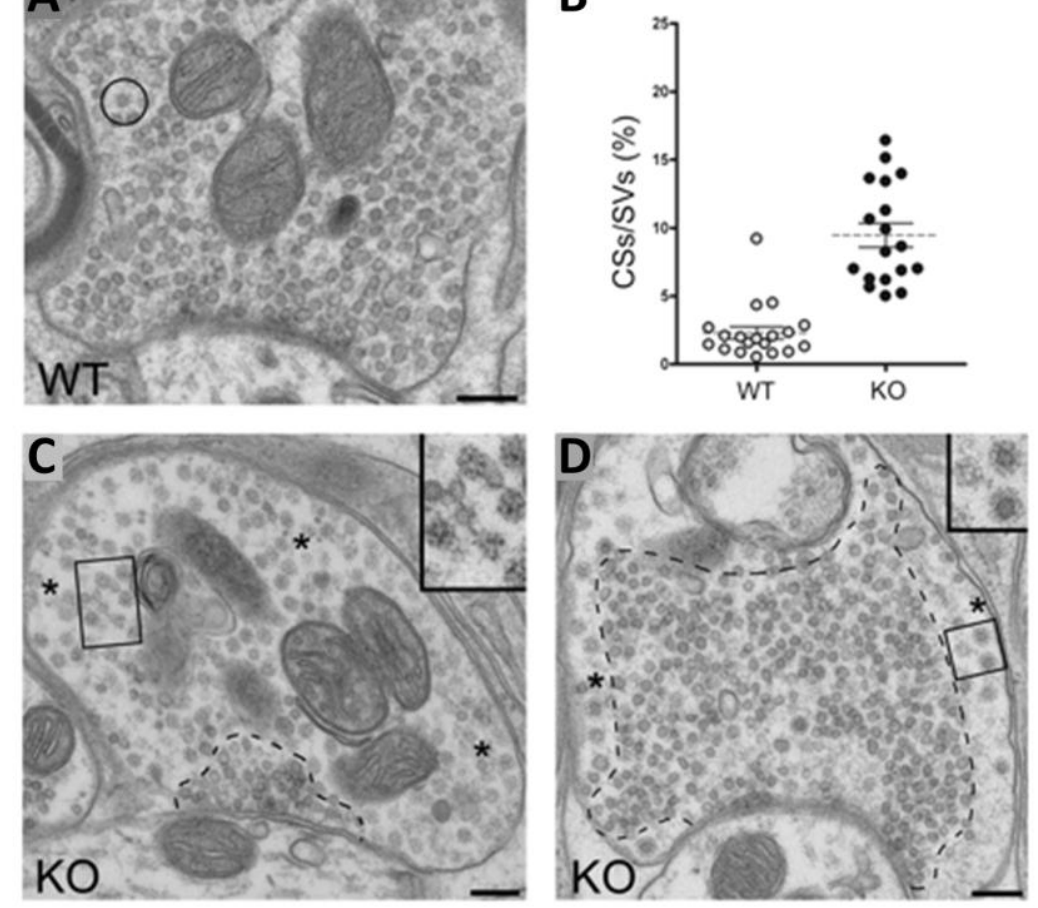

Figure 11 - Accumulation of CCVs in auxilin KO neurons. Electron microscopy images from auxilin WT $(A)$ and $K O(C-D)$ neurons. CCVs and empty clathrin coats accumulate in synaptic terminals as quantified in (B). Scale bars: $200 \mathrm{~nm}$. Illustration from (Yim, et al., 2010). 
auxilin KO mice, the authors report a strong up-regulation of the cyclin-G-dependent kinase (GAK) which is a homologous protein to auxilin that is ubiquitously expressed. Interestingly, the more GAK is up-regulated, the longer auxilin KO mice survive (Yim, et al., 2010), showing that GAK can partially compensate for auxilin loss.

Endocytosis is strongly impaired in neurons from auxilin $\mathrm{KO}$ mice, resulting in an accumulation of CCVs (Figure 11 and Yim, et al., 2010). Moreover, the neurons also display a high abundance of empty clathrin coats. This finding is likely explained by a chaperone function of auxilin for clathrin prevent the formation of such structures in the cytosol. 


\subsection{Protein degradation as an important part of proteostasis}

The process of proteostasis, or protein homeostasis, is an essential process that ensures the constant availability of functional proteins for the diverse cellular tasks. As all processes that occur within the cell require proteins, problems in proteostasis impact virtually every cellular function.

The life time of proteins depends on protein synthesis and folding, trafficking and protein degradation (reviewed in Klaips, Jayaraj, \& Hartl, 2017). Together these processes regulate that proteins are synthesized in appropriate amounts, locate to the respective compartments and are degraded when they are dysfunctional or present at too high levels. Endophilin mutants have recently been reported to display problems in protein degradation (Murdoch, et al., 2016). Degradation is maintained by two distinct processes, autophagy and the ubiquitin-proteasome-system (UPS) that are summarized in this chapter.

\subsubsection{Autophagy}

Autophagy is a well-studied process through which cells degrade cellular structures, including whole organelles, and make the components available again for the generation of new proteins and membranes (Takeshige, Baba, Tsuboi, Noda, \& Ohsumi, 1992; Baba, Takeshige, Baba, \& Ohsumi, 1994). Thereby it ensures the turnover of misfolded, dysfunctional or "aged" proteins and organelles and is critical for the continued abundance of functional proteins. Because of the relevance of autophagy for every cell, the research by Yoshinori Ohsumi that elucidated the molecular mechanism of autophagy was awarded with the Nobel Prize in 2016.

Three distinct mechanisms of autophagy exist: macroautophagy, microautophagy and chaperone mediated autophagy. All of them deliver proteins and organelles to lysosomes where they are degraded by proteases. However, how the structures, that need to be degraded, are recruited is different in the three distinct mechanisms of autophagy. 


\subsubsection{Macroautophagy}

The best studied mechanism of autophagy is macroautophagy, it is therefore often simply referred to as autophagy. Several autophagy-related genes (Atgs) are involved in orchestrating the three steps of macroautophagy. In short, a phagophore is formed and elongated, that proceeds to engulf organelles and cytosolic cargo and finally fuses with a lysosome, where the cargo is degraded. Autophagy is triggered by different signaling mechanisms. Starvation or cellular stresses activate the AMPK pathway that then activates autophagy through an so called ULK complex. Alternatively, the ULK complex can also be activated through growth signals that are mediated by the PI3K-AKT pathway.

Macroautophagy is initiated by the formation of a phagophore, a crescent-shaped membrane structure. The origin of this membrane is subject of on-going debate. The endoplasmic reticulum (ER), the Golgi complex and the plasma membrane have been suggested as a source, while others have suggested a de novo formation from localized synthesis of lipids (reviewed in Reggiori, 2006; and Simonsen \& Tooze, 2009). Some evidence for an ER origin of the membrane was provided by studies that used different imaging techniques to observe phagophore formation (Axe, et al., 2008; Hayashi-Nishino, et al., 2009; Ylä-Anttila, Vihinen, Jokitalo, \& Eskelinen, 2009).

The nucleation of the phagophore is well studied in yeast where the group around Yoshinori Ohsumi discovered a pre-autosomal structure (PAS) consisting of at least 5 Atgs (Atg1, Atg2, Atg5, Atg8 and Atg16; Suzuki, et al., 2001; reviewed in Suzuki \& Ohsumi, 2010). The further formation depends on Atg1 in a complex with Atg13 and Atg17 (see Figure 12). The nucleation of the phagophore in mammalian cells seems to occur without a PAS (reviewed in Klionsky, 2007) and is likely initiated from the ER membrane (Axe, et al., 2008; Hayashi-Nishino, et al., 2009; Ylä-Anttila, Vihinen, Jokitalo, \& Eskelinen, 2009). Still, the mammalian homologue of Atg1, ULK1, is required for the initiation of the process (Kundu \& Thompson, 2008) and works together in a complex with Atg13 and FIP200, the mammalian homologue of Atg17 (Chang \& Neufeld, 2009; Hara \& Mizushima, 2009; Hosokawa, et al., 2009; Jung, et al., 2009). 


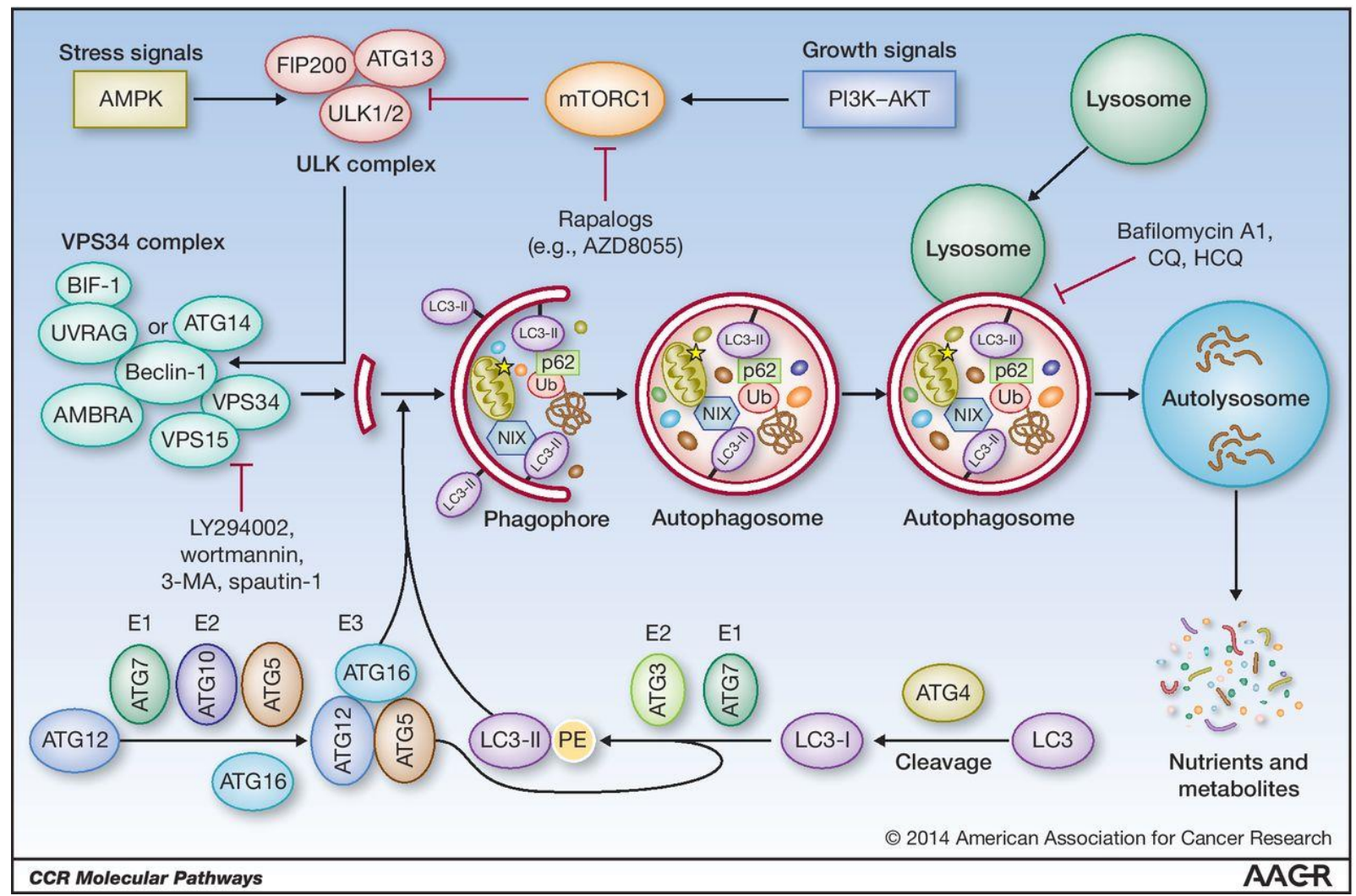

Figure 12- Mechanism of macroautophagy. Autophagy is induced by signaling pathways like the AMPK stress pathway and the PI3K-AKT growth pathway. A large number of Autophagy related proteins (Atgs) is involved in the nucleation and elongation of the phagophore, the maturation of the autophagosome and the subsequent fusion with the lysosome. Illustration from (Cicchini, Karantza, \& Xia, 2015).

Other important factors in the formation process are the vesicular protein sorting 34 (Vps34) and its binding partner beclin-1 (reviewed in Backer, 2008). Vps34 is a PI3 kinase that phosphorylates Pls in the phagophore thereby contributing to its elongation and the recruitment of other Atgs (Jaber \& Zong, 2013; Xie \& Klionsky, 2007). The activity of Vps34 is enhanced through the binding to beclin-1.

After initiation of the phagophore, several Atgs play a role in promoting or inhibiting phagophore maturation. These include ultraviolet irradiation resistant-associated gene (UVRAG), endophilin B1, Atg14L and Bcl-2 (Liang, et al., 2006; Takahashi, et al., 2007; Matsunaga, et al., 2009; Pattingre, et al., 2005). 
The further elongation of the phagophore is mediated by a complex of Atg5, Atg12 and Atg16L in concert with light chain 3 (LC3B). Atg5 and Atg12 are conjugated through the activity of the E1 ubiquitin activating enzyme Atg7 which activates Atg12 using ATP. Atg12 then binds to Atg10, an E2-like ubiquitin carrier that in the next step facilitates the binding to Atg5. The Atg5-Atg12 conjugate then forms a complex with Atg16L (reviewed in Glick, Barth, \& Macleod, 2010). Due to the action of Atg5, which is restricted to the early phase of autophagy, it is often used as a marker for this process. LC3B, on the other hand, is used as a marker for both early and mature phagosomes and autophagosomes.

Atg7, together with Atg3, is also involved in the lipidation of LC3B, in which LC3B is conjugated to phosphatidylethanolamine (PE), a major phospholipid. The lipidated LC3B is recruited to the phagophore by the Atg5-Atg12-Atg16L complex (Fujita, et al., 2008). LC3B is located both on the outer and inner surface of the phagophore and plays a role in selecting cargo. The cargo can be protein aggregates, cytoplasmic components or whole organelles such as mitochondria, ER or Golgi. Most likely, LC3B acts as a receptor that binds to adapter molecules on the cargo (Glick, Barth, \& Macleod, 2010).

Once the cargo is completely engulfed, the phagophore closes due to the action of LC3B. At this point, the structure is called an autophagosome. After closure, LC3B is released from the autophagosome (reviewed in Simonsen \& Tooze, 2009). In the final step, the autophagosome fuses with lysosomes. The fusion is not well studied but relies on the small $G$ protein Rab7 and on Presenilin, a protein highly implicated with Alzheimer 's disease (Eskelinen, 2005).

Due to the low $\mathrm{pH}$ in the lysosomes and the proteases inside, the autophagosome cargo is degraded. These proteases include cathepsin B and D (Koike, et al., 2005).In addition, the lysosomal protein LAMP1 and LAMP2 are important for the degradation, possibly by maintaining the $\mathrm{pH}$ in the autolysosome (Tanaka, et al., 2000).

Nutrients and metabolites that are the product of the degradation are finally released to the cytoplasm where they are available for protein synthesis and other cellular processes. 


\subsubsection{Microautophagy}

Microautophagy refers to the degradation of cytoplasmic components and organelles without the formation of an autophagosome. It was originally described in yeast as the direct invagination of lysosomal membrane to capture the cytoplasm and its contents (Kunz, Schwarz, \& Mayer, 2004; Uttenweiler, Schwarz, Neumann, \& Mayer, 2007; reviewed in Tekirdag \& Cuervo, 2017). In mammalian cells, microautophagy does not happen immediately at the lysosome, but rather at late endosomes (LE) and/or multi-vesicular bodies (MVB) and has thus been termed endosomalmicroautophagy (eMl; Sahu, et al., 2011). LEs are large endosomal structures that mature into lysosomes (Piper \& Luzio, 2001).

The internalization of cargo for degradation is mediated through the ESCRT machinery which is present on LE. Several components of this machinery have been shown to be necessary for eMI (Sahu, et al., 2011; Mukherjee, Patel, Koga, Cuervo, \& Jenny, 2016). Microautophagy degrades proteins either unspecifically "in bulk" or selectively if the proteins contain a Lys-Phe-Glu-Arg-Gln (KFERQ) motif (Dice, 1995). However, this motif is not sufficient to target proteins for microautophagy (Koga, Kaushik, \& Cuervo, 2011).

The degradation can occur either directly in the LE or after fusion of the LE with a lysosome (Sahu, et al., 2011; Mukherjee, Patel, Koga, Cuervo, \& Jenny, 2016). The degradation and final products are similar to the process of macroautophagy.

\subsubsection{Chaperone mediated autophagy}

The third process of autophagy is called chaperone-mediated autophagy as it relies on cargo selection by a chaperone. Unlike macroautophagy, the cargo is directly recruited to the lysosomes where it is subsequently degraded (Tekirdag \& Cuervo, 2017).

The selection of cargo is mediated by the chaperone Hsc70, which also plays a role in the uncoating of clathrin-coated vesicles (s. chapter 1.2.1). It binds to proteins carrying a KFERQ motif (Dice, 1995). This motif is sufficient to target proteins for chaperone-mediated autophagy (Sahu, et al., 2011; Koga, Kaushik, \& Cuervo, 2011). 
Hsc70 then binds to the cytosolic tail of LAMP2A on the lysosomal membrane and thereby transports the cargo to the site of degradation. After binding, the protein is unfolded by Hsc70. Unfolding is necessary for successful degradation of the protein (Salvador, Aguado, Horst, \& Knecht, 2000). After unfolding, the target protein is transported into the lysosome where it is then degraded.

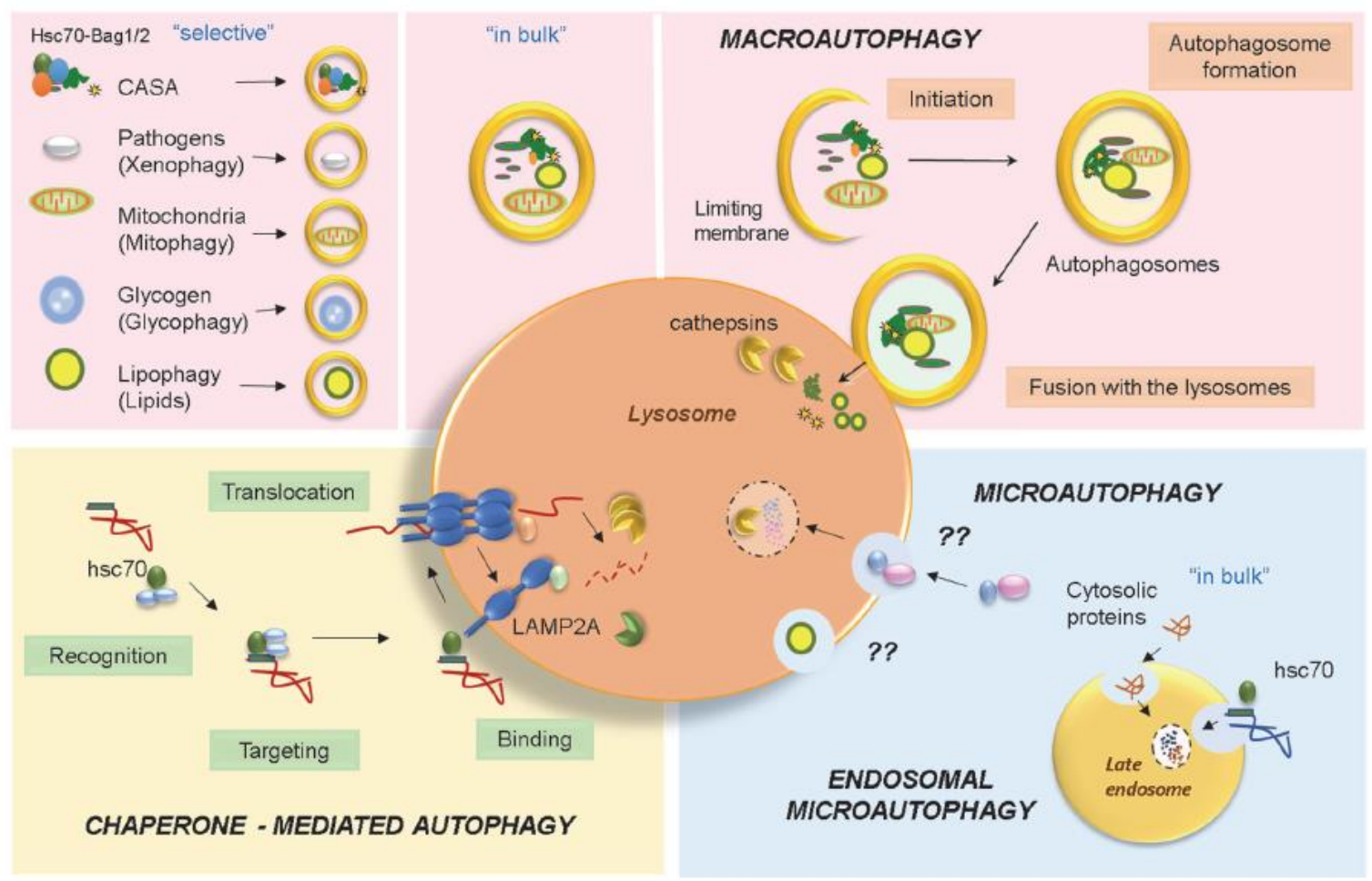

Figure 13 - Modes of Autophagy. Graphic summary of macroautophagy, microautophagy and chaperone-mediated autophagy from (Tekirdag \& Cuervo, 2017). 


\subsubsection{The Ubiquitin-Proteasome-System}

An alternative, or rather, complementary way to regulate proteostasis is the UbiquitinProteasome-System (UPS). While autophagy is thought to target long-living proteins to adept to extracellular influences like starvation, the UPS targets short-living proteins and is believed to be more important for intracellular processes (Rock, et al., 1994; Lee \& Goldberg, 1996; Fuertes, Martín De Llano, Villarroya, \& Knecht, 2003).

The system works through the proteasome, a complex of over 40 subunits which is located in the nucleus and in the cytoplasm. The proteasome consists of two main components: the $20 \mathrm{~S}$ core particle and the 19S regulatory particle (Figure 14; Coux, Tanaka, \& Goldberg, 1996). The core particle is made up of two inner a-rings and two outer $\beta$-rings that together form a barrel-like structure and consist of proteases (Groll, et al., 1997; Chowdhury \& Enenkel, 2015). The regulatory particle is composed of a base and a lid that together select the substrates for the proteasome (Rosenzweig, Osmulski, Gaczynska, \& Glickman, 2008; Chowdhury \& Enenkel, 2015).

The selection of substrates for the UPS occurs through a number of enzymes classed as E1, E2 and E3 that selectively add poly-ubiquitin chains to proteins to mark them

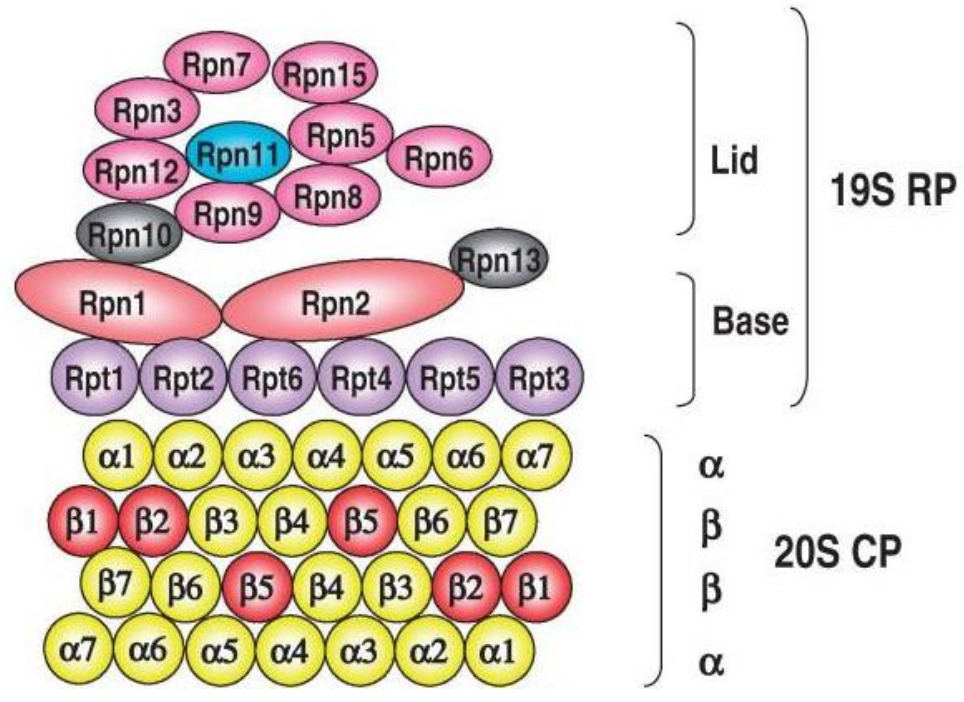

Figure 14 - Structure of the 26S proteasome. The proteasome consists of a $20 \mathrm{~S}$ core particle and a 19S regulatory particle. CP: core particle, RP: regulatory particle, Rpn: RP non-ATPase, Rpt: RP triple-ATPase, $\alpha 1-7$ : members of the inner $\alpha$-ring, $\beta 1$ 7: members of the outer $\beta$-ring. Adapted from (Tanaka, 2009). 
for proteasomal degradation. These enzymes are responsible for ubiquitin activation, ubiquitin conjugation and ubiquitin ligation respectively (Hershko \& Ciechanover, 1998). Briefly, ubiquitin is activated in an ATP-dependent manner by the E1 activating protein and then transferred to the cysteine residue of an E2 conjugating enzyme. In the last step, the ubiquitin is transferred via the E3 ligase to a lysine residue of a target protein.

In order for a protein to be marked for degradation, it is loaded with several ubiquitin proteins. Ubiquitin itself carries seven lysines (K6, K11, K27, K29, K33, K48 and K63; Ben-Saadon, Zaaroor, Ziv, \& Ciechanover, 2006). However, poly-ubiquitination of the different lysine residues causes different effects. Poly-ubiquitination on lysine residue K48 of the first and subsequent ubiquitin proteins marks a protein for degradation, while ubiquitination of $\mathrm{K} 63$ has effects on trafficking and other cellular processes (Sadowski, Suryadinata, Tan, Roesley, \& Sarcevic, 2012; Pickart \& Fushman, 2004; Ben-Saadon, Zaaroor, Ziv, \& Ciechanover, 2006; Silva, Finley, \& Vogel, 2015). Importantly, a large number of E3 ubiquitin ligases ensures the specificity of the process: While there are only a few known E1 enzymes (Liu, Goldberg, \& Qiu, 2007), there are at least $40 \mathrm{E} 2$ enzymes in humans (Valimberti, Tiberti, Lambrughi, Sarcevic, \& Papaleo, 2015; Wenzel, Hunsaker, Greco, Willemsen, \& Berman, 2010; Burroughs, Jaffee, lyer, \& Aravind, 2008) and several hundred E3 ubiquitin ligases (Zheng \& Shabek, 2017; Sluimer \& Distel, 2018).

Of note, there are E3 ubiquitin ligases that can function alone, as the family of homologous to the E6AP carboxyl terminus (HECT) domain proteins or of the really interesting new gene (RING) family (Metzger, Pruneda, Klevit, \& Weissman, 2014), while others function in a complex (Hershko \& Ciechanover, 1998). The best studied example for such a complex is the Skp1, Cullin1, F-box protein complex (SCF; Skowyra, Craig, Tyers, Elledge, \& Harper, 1997). The principle of substrate polyubiquitination of the SCF complex and a RING protein is illustrated in Figure 15.

K48 poly-ubiquitinated proteins are subsequently recognized by the regulatory particle of the proteasome. It consists of 19 subunits that can be classified into "regulatory particle triple-ATPase" (Rpt) and "regulatory particle non-ATPase" (Rpn) subunits (Tanaka, 2009). Rpn10 and Rpn13 are located on the base of the regulatory 

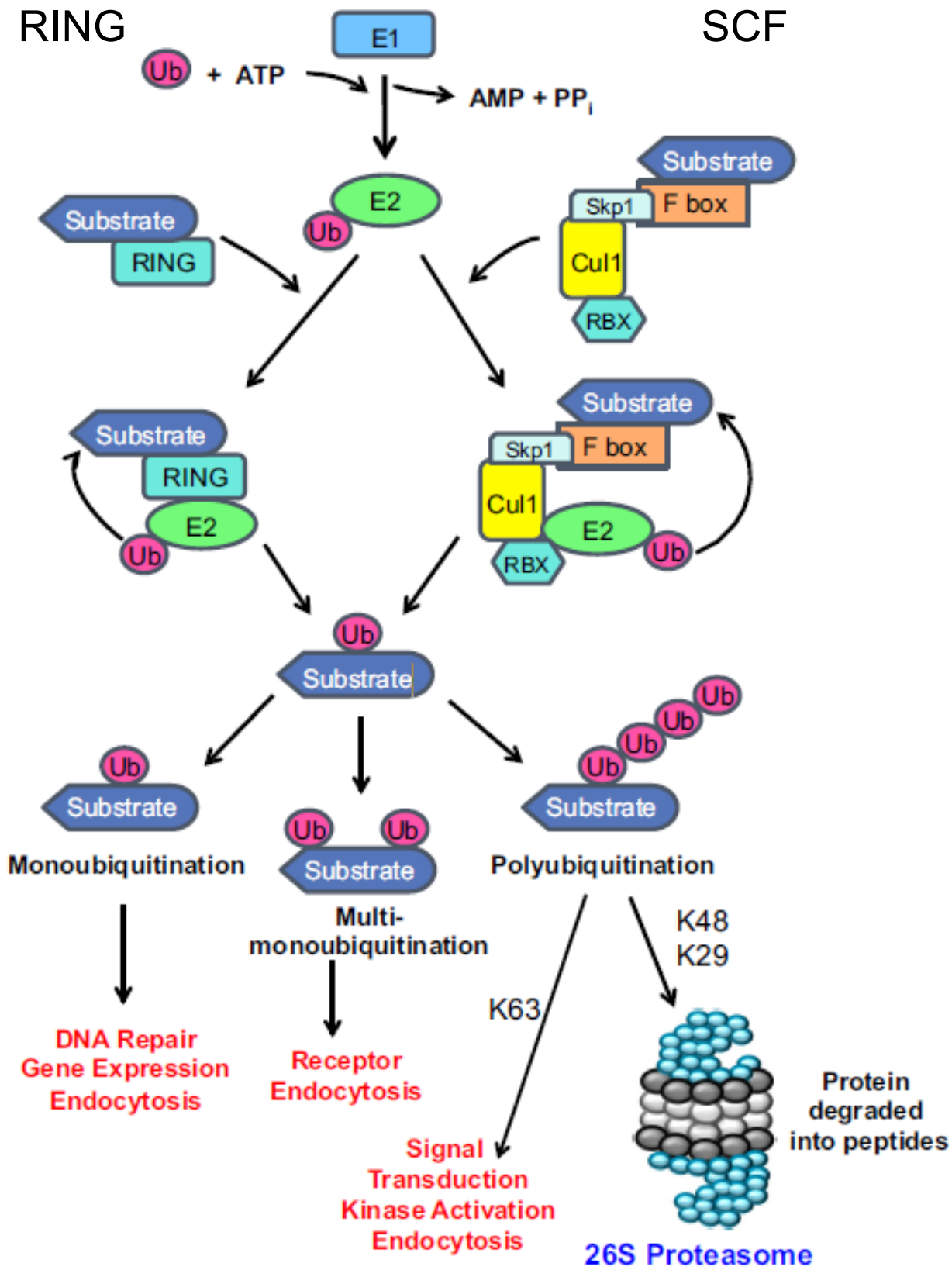

Figure 15 - Substrate poly-ubiquitination through a RING protein or the SCF complex. In both pathways, ubiquitin is activated by an E1 ubiquitin-activating protein and subsequently bound by an E2 conjugating protein. In the RING protein pathway, the substrate and the E2 protein are both bound to the RING protein and the ubiquitin is then transferred to the target protein (left). In case of the SCF complex, the target protein is bound by the F-box protein, which is conjugated to Skp1, Cullin1 (Cul1) and the RING E3 ubiquitin ligase RBX. The E2 ubiquitin conjugating protein is bound by RBX and Cul1 which then ligase ubiquitin to the target protein (right). The ubiquitinated protein is either mono-ubiquitinated, multi-mono-ubiquitinated (at different lysine residues) or poly-ubiquitinated. Proteasomal degradation only occurs following K29 and K48 poly-ubiquitination. From (Bodine \& Baehr, 2014). 
particle and function as receptors for ubiquitin, thereby directing poly-ubiquitinated proteins to the proteasome (Husnjak, et al., 2008; Schreiner, et al., 2008). The polyubiquitin chain is then removed from the target protein by the deubiquitinating enzyme Rpn11 (Ventii \& Wilkinson, 2008). The target protein is ultimately degraded through catalytic enzymes in the core particle of the proteasome into oligopeptides of 3-15 amino acids that are further degraded by oiligopeptidases (Tanaka, 2009; Groll, et al., 1997; Chowdhury \& Enenkel, 2015). 


\subsection{Fbxo32, an E3 Ubiquitin-Ligase involved in autophagy}

One example for an E3 ubiquitin-ligase is the F-box only protein 32 (Fbxo32, also called MAFbx or atrogin-1, UniProtKB: Q969P5). It was first discovered by two independent studies in 2001 as a regulator of muscle atrophy (Bodine, et al., 2001; Gomes, Lecker, Jagoe, Navon, \& Goldberg, 2001). In these studies, Fbxo32 was reported to be expressed in muscles and severely up-regulated upon several conditions, including fasting, denervation, immobilization and diabetes. Fbxo32 was recently also found to be upregulated in endophilin mutants (see Figure 8 and Murdoch, et al., 2016).

\subsubsection{Structure of Fbxo32}

Fbxo32 is a 355 amino acid protein that is well conserved between human and mouse and contains several functional domains (Gomes, Lecker, Jagoe, Navon, \& Goldberg, 2001; Csibi, Tintignac, Leibovitch, \& Leibovitch, 2008; Julie, Sabrina, Marie-Pierre, \& Leibovitch, 2012). It is located in the cytoplasm and is also present in the nucleus due to two nuclear localization sequences (NLS) and a nuclear export sequence (NES) contained in a leucine charged domain (LCD; Figure 16; Julie, Sabrina, Marie-Pierre, \& Leibovitch, 2012).

Fbxo32 was found to be part of an E3 ubiquitin ligase complex together with Skp1, Cullin1 and RBX forming a SCF complex (Gomes, Lecker, Jagoe, Navon, \& Goldberg, 2001; Skowyra, Craig, Tyers, Elledge, \& Harper, 1997). It can bind both Skp1 and Cullin1 through an F-box domain in the central part of the protein, which also is responsible for the specific substrate binding for the E3 ubiquitin ligase function (Bai, et al., 1996; Bodine, et al., 2001; Skowyra, Craig, Tyers, Elledge, \& Harper, 1997; Bodine \& Baehr, 2014). However, Fbxo32 has no WD40 repeats or leucine-rich regions which are often found in other F-box proteins (Figure 16; Winston, et al., 1999; Cenciarelli, et al., 1999).

Besides the name giving F-box domain, Fbxo32 also possesses a leucine zipper domain (LZ), a cytochrome c heme binding site (cytC) and a PDZ binding domain at 
the C terminus (Figure 16; Bodine, et al., 2001; Gomes, Lecker, Jagoe, Navon, \& Goldberg, 2001; Csibi, Tintignac, Leibovitch, \& Leibovitch, 2008; Julie, Sabrina, Marie-Pierre, \& Leibovitch, 2012). As Fbxo32 has no WD40 or leucine rich domain, the PDZ binding domain likely functions to bind specific complementary sequences on target proteins for poly-ubiquitination (Doyle, Lee, Lewis, Kim, \& MacKinnon, 1996).

$\begin{array}{rrrrr}10 & 20 & 30 & 40 & 50 \\ \text { MPFLGQDWRS } & \text { PGQNWVKTAD } & \text { GWKRFLDEKS } & \text { GSFVSDLSSY } & \text { CNKEVYNKEN } \\ 60 & 70 & 80 & 90 & 100 \\ \text { LFNSLNYDVA } & \text { AKKRKKDMLN } & \text { SKTKTQYFHQ } & \text { EKWIYVHKGS } & \text { TKERHGYCTL } \\ 110 & 120 & 130 & 140 & 150 \\ \text { GEAFNRLDFS } & \text { TAILDSRRFN } & \text { YVVRLLELIA } & \text { KSQLTSLSGI } & \text { AQKNFMNILE } \\ 160 & 170 & 180 & 190 & 200 \\ \text { KVVLKVLEDQ } & \text { QNIRLIRELL } & \text { QTLYTSLCTL } & \text { VQRVGKSVLV } & \text { GNINMWVYRM } \\ 210 & 220 & 230 & 240 & 250 \\ \text { ETILHWQQQL NNIQITRPAF } & \text { KGLTFTDLPL } & \text { CLQLNIMQRL } & \text { SDGRDLVSLG } \\ 260 & 270 & 280 & 290 & 300 \\ \text { QAAPDLHVLS } & \text { EDRLLWKKLC } & \text { QYHFSERQIR } & \text { KRLILSDKGQ } & \text { LDWKKMYFKL } \\ 310 & 320 & 330 & 340 & 350 \\ \text { VRCYPRKEQY } & \text { GDTLQLCKHC } & \text { HILSWKGTDH } & \text { PCTANNPESC } & \text { SVSLSPQDFI }\end{array}$

NLFKF

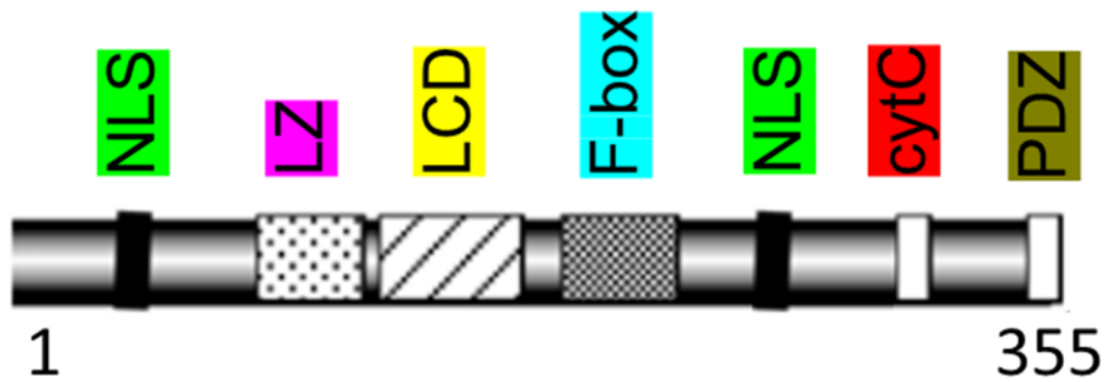

Figure 16 - Sequence and domain structure of Fbxo32. Amino acid sequence (top) and domain representation (bottom) of human Fbxo32. Fbxo32 contains two nuclear localization sequences (aa62-67 and aa280-295; green), a leucine zipper motif (aa99135; pink), a leucine charged domain containing a nuclear export sequence (aa140190; yellow), an F-box domain (aa228-267; turquoise), a cytochrome-c site (aa317322; red) and a PDZ binding domain (aa346-355; dark green). Adapted from (Julie, Sabrina, Marie-Pierre, \& Leibovitch, 2012). 


\subsubsection{Role and targets of Fbxo32 in proteasomal degradation}

Fbxo32 functions as an E3 ubiquitin-ligase and thus selects targets for polyubiquitination and subsequent degradation by the UPS (s. chapter 1.5.5). As Fbxo32 has been reported as a mediator of muscle atrophy, many studies on this protein were originally conducted in the context of muscle development and disease.

As such, many interaction partners and E3 ubiquitin ligase targets were identified in this area including the myoblast determination protein (MyoD; Tintignac, et al., 2005) and myogenin (Jogo, Shiraishi, \& Tamura, 2009; Foletta, White, Larsen, Léger, \& Russell, 2011), two transcription factors that are involved in muscle differentiation (Davis, Weintraub, \& Lassar, 1987; Sassoon, et al., 1989; Edmondson \& Olson, 1993), the eukaryotic translation initiation factor 3 subunit $f$ (elF3-f; LagirandCantaloube, et al., 2008), that plays a role in mTOR dependent muscle differentiation (Csibi, et al., 2010), two sacromeric proteins, vimentin and desmin (Lokireddy, et al., 2012; Bodine \& Baehr, 2014), the dual specificity protein phosphatase 1 (DUSP1; Xie, et al., 2009) a regulator of the skeletal muscle development kinase, mitogen-activated protein kinase 1 (MAPK1; Keren, Tamir, \& Bengal, 2006) and calcineurin which promotes cardiac hypertrophy in response to pathologic stimuli in cardiomyocytes ( $\mathrm{Li}$, et al., 2004; Willis \& Patterson, 2006).

However, targets of Fbxo32 mediated ubiquitination were also found in the context of other cellular processes. These include c-Myc, a transcription factors that works as an oncogene (Mei, et al., 2015), krueppel-like factor 4 (KLF4), a transcription factor active during embryonic development (Zhou, et al., 2017), the charged multivesicular body protein 2B (CHMP2B) which plays a role in autophagy (Zaglia, et al., 2014) and CtBP1 which is involved in epithelial to mesenchymal transition (EMT) and dynaminindependent endocytosis (Sahu SK1, et al., 2017; Amstutz, et al., 2008; Bonazzi, et al., 2005).

Lastly, Fbxo32 seems to have a role in the regulation of apoptosis and cell survival. It was shown to play a direct role in the NF-KB signaling pathway by targeting the inhibitor of the transcription factor ReIA, IKBa, to proteasomal degradation (Meshram, et al., 2017). The NF-KB pathway is ubiquitously expressed and regulates inflammation, apoptosis, oncogenesis and proliferation (Mincheva-Tasheva \& Soler, 
2013). The inhibitor IKBa, which is the most common inhibitor of NF-KB in the nervous system, retains the transcription factor in the cytoplasm and its degradation through the 26S proteasome is part of the mechanism of the signal transduction (Chen, 2005; Mincheva-Tasheva \& Soler, 2013). By targeting IкBa for proteasomal degradation, Fbxo32 functions directly in the regulation of the NF-kB signaling pathway.

Interestingly, two of the above mentioned Fbxo32 targets, DUSP1 and KLF4, were also implicated in the regulation of cell survival. DUSP1 de-phosphorylates the stressactivated protein kinase JNK (Zhou, et al., 2017; Xie, et al., 2009), which regulates apoptosis and autophagy by phosphorylation of specific transcription factors (Sui, et al., 2014; Xie, et al., 2009). KLF4 was also shown to induce apoptosis upon oxidative stress in chronic myeloid leukemia cells (Li, et al., 2010)

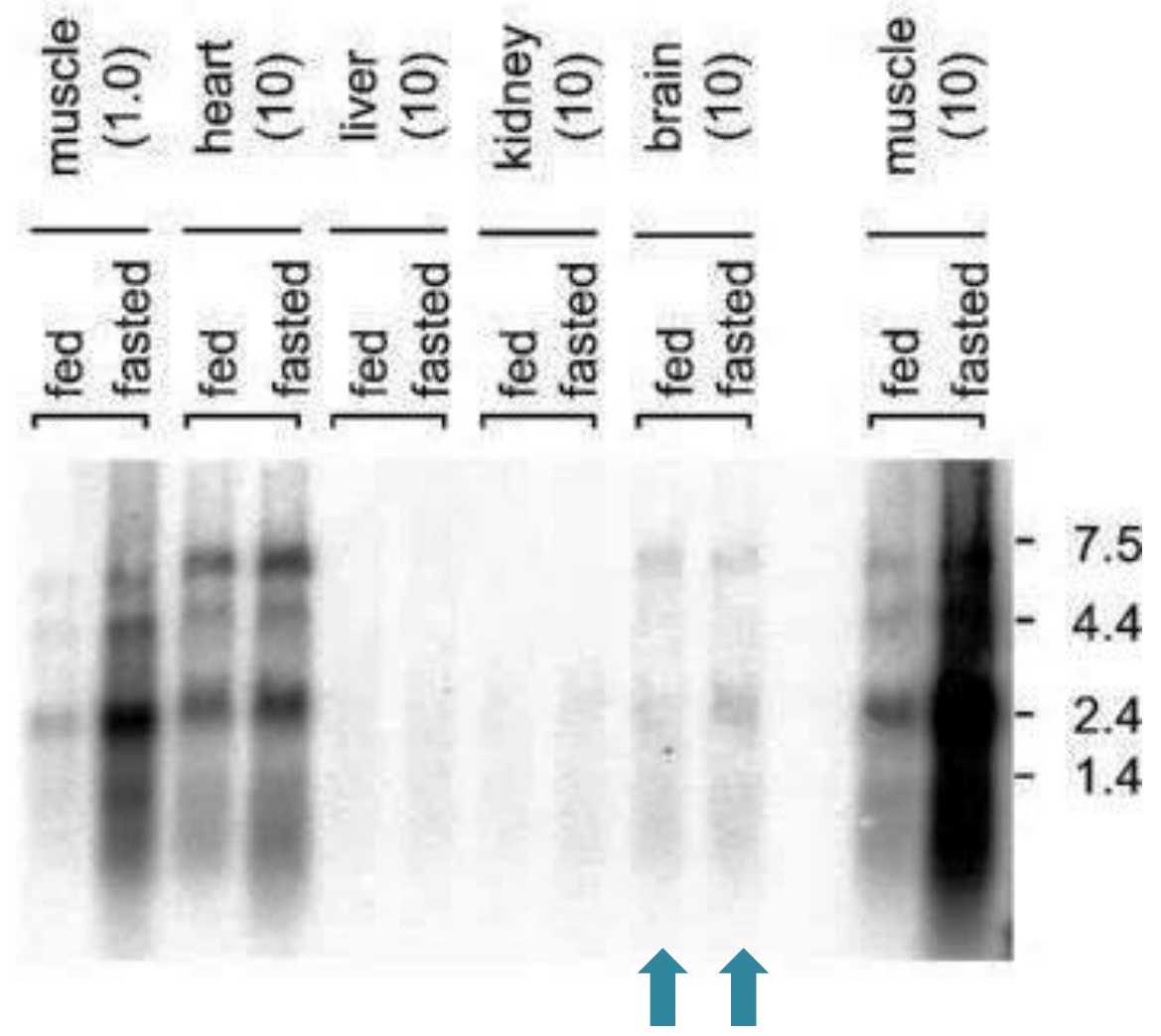

Figure 17 - Expression of Fbxo32 in different tissues upon starvation. RNA hybridization for Fbxo32 in muscle, heart, liver, kidney and brain samples of fed and starved mice. Brackets show amount of loaded RNA in micrograms. While Fbxo32 is most strongly expressed in muscle tissue, it is also found in a lower quantity in the brain samples (arrows). Modified from (Gomes, Lecker, Jagoe, Navon, \& Goldberg, 2001). 
Taken together, these targets of Fbxo32 dependent poly-ubiquitination and subsequent proteasomal degradation point to a role of Fbxo32 in muscle differentiation and atrophy as well as general cell survival. While Fbxo32 is strongly expressed in skeletal muscles, it was also found to be expressed in the central nervous system (Figure 17; Gomes, Lecker, Jagoe, Navon, \& Goldberg, 2001). Given the above mentioned functions of Fbxo32 in muscles, this thesis hypothesizes that Fbxo32 might play a similar role in regulating cell survival in the brain.

\subsubsection{Fbxo32 as a mediator between autophagy and the UPS}

After its discovery as a muscle-enriched E3 ubiquitin ligase, Fbxo32 was further studied in mouse and zebrafish animal models (Bodine, et al., 2001; Zaglia, et al., 2014; Bühler, et al., 2016). In the acute model of muscle denervation, Fbxo32 KO had a positive effect in preventing muscle degeneration in mice (Bodine, et al., 2001). Further studies showed that Fbxo32 was up-regulated in models of acute muscle atrophy and that its down-regulation had a positive effect on muscle survival (Sakai, et al., 2017; Lee, Kim, Kim, Choi, \& Nam, 2017).

However, genetic ablation also resulted in an age-dependent increase on cardiomyopathy in mice (Zaglia, et al., 2014) and progressive heart failure and myopathy in zebrafish (Bühler, et al., 2016).

Furthermore, the Fbxo32 KO models imply that Fbxo32 plays a role in autophagy in addition to its described role in the UPS (see chapter 1.6.2). Zaglia and colleagues report that a member of the endosomal sorting complex (ESCRT), charged multivesicular body protein $2 \mathrm{~b}$ (CHMP2B), is a target of Fbxo32 mediated polyubiquitination (Zaglia, et al., 2014). The ESCRT complex is involved in the formation of multi-vesicular bodies (MVB, see also chapter 1.5.3) which can fuse with autophagosomes before the final fusion with the lysosome, thereby contributing in targeting the proteins engulfed in the MVBs for degradation (Fader \& Colombo, 2009). Using the Fbxo32 KO mouse model, they show that CHMP2B accumulates in the heart due to inefficient degradation. They hypothesize that this accumulation leads to 
autophagy impairment which in turn causes the observed phenotype of cardiomyopathy.

Supporting this hypothesis, a mouse model lacking Atg5, a key factor for autophagy (see chapter 1.5.2), displayed a similar phenotype of age-dependent cardiomyopathy (Taneike, et al., 2010). In addition, Bühler and colleagues showed that in their zebrafish KO model the autophagy marker LC3B is strongly up-regulated and that autophagy inhibition had no additional effect in fish missing Fbxo32 (Bühler, et al., 2016).

In summary, Fbxo32 is strongly up-regulated in models of starvation, denervation, immobilization or diabetes and seems to play a role in the induction of muscle atrophy likely through targeting key muscle regulators for degradation. On the other hand, genetic ablation of Fbxo32 in mouse or zebrafish animal models also results in myopathy, showing that both overexpression and deletion of Fbxo32 can potentially have negative effects for the organism. Because of that, and the implication of Fbxo32 to autophagy, Fbxo32 has been proposed to act as a mediator between UPS dependent protein degradation and autophagy (Zaglia, et al., 2014).

This thesis will further explore the importance of Fbxo32 levels and its role in autophagy. 


\subsection{Neurodegenerative diseases}

Neurodegenerative diseases are a cluster of human pathological conditions that primarily affect neurons. These diseases are not only a problem because of their increasing prevalence and expected health costs, but also because they reduce the live quality of affected patients dramatically. In the final stages of the disease, many patients require intense nursing for even the most basic daily tasks and the memory loss is a burden for both the patient and his or her family. While the symptoms of the disease can be treated in many cases, dead neurons cannot be regenerated, so brain functions that have been lost can generally not be restored.

A variety of human conditions, including Alzheimer's Disease (AD), Parkinson's Disease (PD) and Huntington's Disease (HD), are classified as such diseases. While all neurodegenerative diseases are characterized by neuronal death, the location and magnitude of the cell death differs and is responsible for the different symptoms that define the specific disease. For example, in the case of PD the most affected cell type is dopaminergic neurons. These neurons are enriched in the substatia nigra pars compacta. This is a small brain region in the midbrain important for motor planning and eye movement. Because of this, the most obvious phenotype in PD patients, which can be easily observed even with an untrained eye, is motor deficits, particularly tremor in the hands and gait anomalies.

As many studies have investigated neurodegenerative diseases, several hypotheses have been formulated about the causes for such diseases. Many proteins have been identified, that - when mutated or dysregulated - contribute to neurodegeneration. These proteins include amyloid- $\beta$ and tau for AD, $\alpha$-synuclein and LRRK for PD as well as huntingtin, ataxin-2, superoxide dismutase 1 and prion proteins for several other diseases. Interestingly, many of these proteins accumulate in context of the specific diseases and form cytotoxic aggregates (Bayer, 2015). In light of this, an emerging hypothesis is that problems in proteostasis could contribute to neurodegeneration or even be a common denominator of several neurodegenerative conditions (Douglas \& Dillin, 2010; Nixon, 2013; Liang \& Sigrist, 2018). As 
proteostasis is an essential function for all cellular processes (see chapter 1.5), it is not surprising that impairments in proteostasis may also impact neuronal survival.

According to this hypothesis, the reason why neurons are especially vulnerable to problems with proteostasis is their specific architecture. Due to the elongated shape of neurons with long axons, synapses are often a significant distance away from the nucleus and the protein synthesis machinery that can at least partially compensate for impairments in protein degradation. This deteriorates even further with aging, as the proteostasis system loses effectiveness with age (reviewed in Cuervo, et al., 2005; Martinez-Vicente, Sovak, \& Cuervo, 2005). Protein aggregates in the synapse would then cause the degeneration of synapses which consequently causes axonal retraction and finally neuronal death (Burke \& O'Malley, 2013).

\subsubsection{Defective endocytosis and neurodegeneration}

In the past years, several studies have been published that link defects in endocytosis to neurodegeneration. These studies encompass a variety of approaches and originate from groups working on diverse aspects of neurodegeneration. In their totality it is intriguing how strongly they connect defective endocytosis to neurodegenerative diseases.

For example, protein interaction studies have shown that endophilin interacts with several proteins that are strongly associated with neurodegenerative disorders (Ralser, et al., 2005). The authors showed that endophilin 1 and 3 interact with ataxin2. Mutations in the gene encoding ataxin-2, SCA2, have been identified as the cause of spinocerebellar ataxia type 2 (Sanpei, et al., 1996).

Endophilin has also been shown to interact with LRRK2 (Matta, et al., 2012), mutations of which are the most common cause for familiar and sporadic PD (Nichols, et al., 2005; Di Fonzo, et al., 2006; Gilks, et al., 2005; reviewed in Kumari \& Tan, 2009). Interestingly, recent studies report that LRRK2 might play a role in the regulation of endophilin in CME through the phosphorylation of the insertion site of endophilin's amphipathic a-helices (Ambroso, Hegde, \& Langen, 2014; Soukup, et al., 2016). This is further supported by another study of LRRK2 KO mice which reports 
defects in neurotransmission and endocytosis in neurons from these mice (Arranz, et al., 2015).

The link to PD is solidified by a study that shows a direct binding between parkin, another key protein in PD (reviewed in Dawson \& Dawson, 2010), and all three endophilins (Trempe, et al., 2009). Moreover, PD disease progression was shown to correlate with an accompanying increase of endophilin 1 in the cortex of PD patients (Shi, et al., 2009). In addition to this, a number of patients with juvenile or early-onset PD have been reported to carry mutations of genes important to endocytosis, more precisely the uncoating process: synaptojanin-1 (Quadri, et al., 2013; Krebs, et al., 2013; Olgiati, et al., 2014) and auxilin (Edvardson, et al., 2012; Köroğlu, Baysal, Cetinkaya, Karasoy, \& Tolun, 2013; Olgiati, et al., 2016). On the other hand, endophilin was also reported to be up-regulated in the brains of AD patients, possibly pointing to a more basic role of endophilin in several neurodegenerative diseases (Ren, et al., 2008).

The creation of animal models for defective endocytosis has made it possible to further elucidate the connection to neurodegenerative disorders. Endophilin DKOs were reported to display symptoms of cerebellar ataxia which is likely caused by the retraction of climbing fibers from the inferior olivary nucleus to the cerebellum (Milosevic, et al., 2011). In the same model, it was later reported that parkin is upregulated in both endophilin TKO and p18 DKO mice (Cao, Milosevic, Giovedi, \& De Camilli, 2014).

In addition, a fly model lacking endophilin in the eye was shown to display photoreceptor cell degeneration. In the same study, the authors report that the balancing of phosphorylation of endophilin through LRRK2 is important to maintain neuronal survival in dopaminergic neurons (Soukup, et al., 2016).

Recently, a mouse model was published that carries a mutation of synaptojanin-1 that was identified in patients of early-onset Parkinsonism (Cao, et al., 2017; Quadri, et al., 2013; Krebs, et al., 2013; Olgiati, et al., 2014). These mice display changes in the structure of dopaminergic synapses in the dorsal striatum (Cao, et al., 2017). A second study in a fly model of the same mutation reported dopaminergic neuron 
degeneration, emphasizing the connection of the synaptojanin-1 mutation to PD (Vanhauwaert, et al., 2017).

The cause for the neurodegeneration in endocytic mutants is not clear yet. One theory is that defects in endocytosis will ultimately lead to defects in exocytosis, as exocytosis requires the continuous availability of SVs. This process is likely age dependent, as basic synaptic transmission is still possible in all presented models of defective endocytosis and only becomes impaired upon strong stimulation (Milosevic, et al., 2011; Cremona, et al., 1999; Cao, et al., 2017; Raimondi, et al., 2011; Yim, et al., 2010). Once exocytosis is impaired, the synaptic strength, both pre- and postsynaptically, is weakened, causing synapses to disappear. As a result, axons will retract, which is an early hallmark of neurodegeneration (Burke \& O'Malley, 2013; Schirinzi, et al., 2016).

However, some recent studies in this area report that loss or mutations of endocytic proteins causes defects in autophagy and hypothesize this as a possible cause for the observed neurodegeneration (Soukup, et al., 2016; Vanhauwaert, et al., 2017). In this scenario, the defect in autophagy would impair proteostasis in synapses which are de facto isolated from the nucleus and the protein synthesis machinery due to the long distance between them. The imbalance in proteostasis would then result in a disruption of synaptic processes with the same consequences as detailed above.

\subsubsection{Autophagy impairments and neurodegeneration}

Defects in protein homeostasis and protein aggregation are common hallmarks of both aging and neurodegeneration (Martin, Austad, \& Johnson, 1996; Kumar, et al., 2016). It is therefore not surprising that defects in autophagy are often observed in the context of neurodegenerative diseases (Kumar, et al., 2016). This is prominently observed in tauopathies, including $A D$ and other neuropathies such as PD, HD and Creutzfeldt-Jakob/prion diseases (Lee \& Leugers, 2012; Giráldez-Pérez, AntolínVallespín, Muñoz, \& Sánchez-Capelo, 2014; Davies, et al., 1997; Cescatti, et al., 2016). The formed protein aggregates, which are not degraded by autophagy, have 


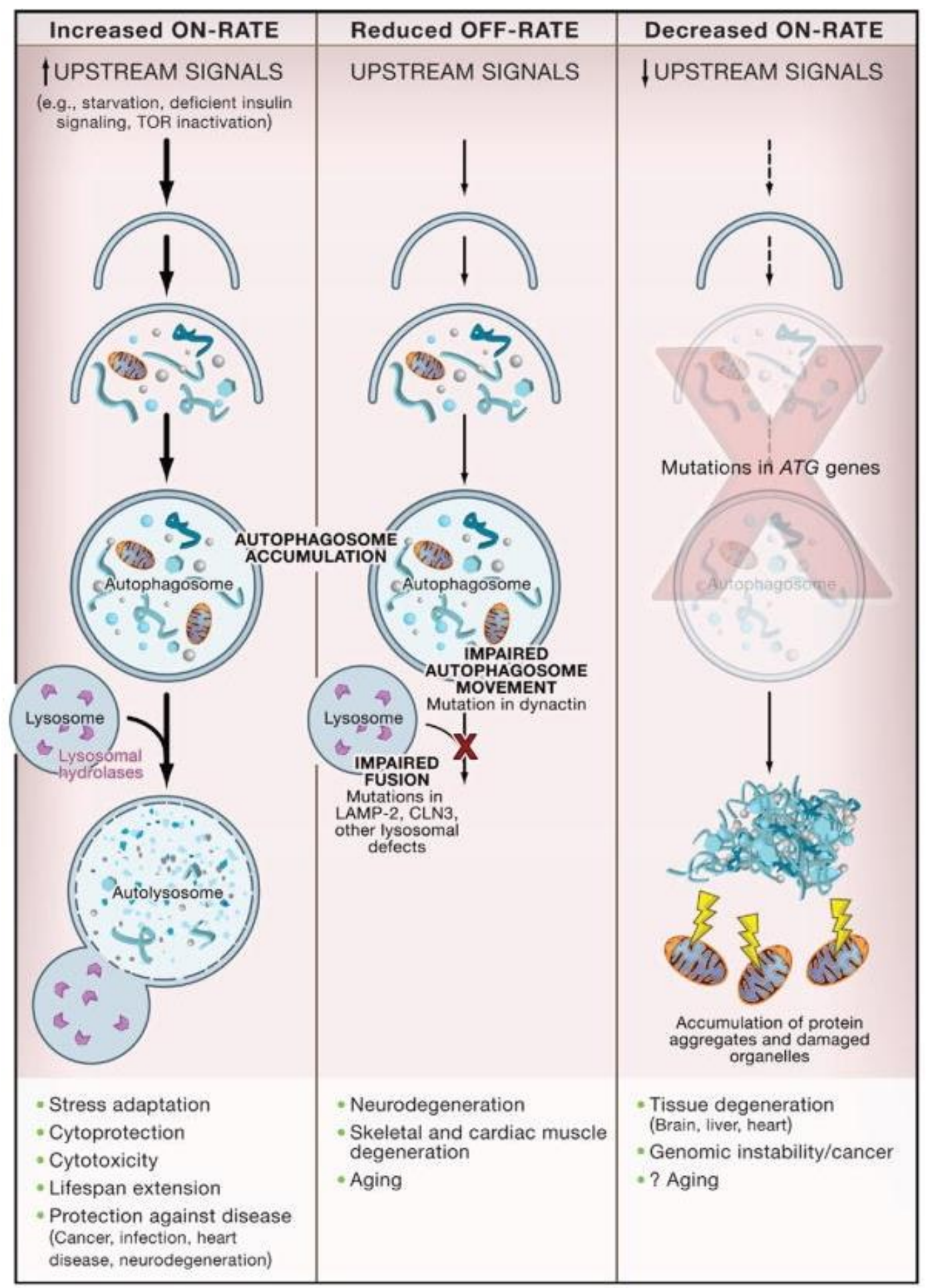

Figure 18 - Consequences of defective autophagy. Up-regulation of autophagy is a cellular mechanism for adaptation to stress and cytotoxicity and has protective consequences (left). Defective fusion of autophagosomes with lysosomes leads to neurodegeneration and premature aging (middle). Impaired autophagosome formation causes tissue degeneration (right). Illustration from (Levine \& Kroemer, 2008). 
cytotoxic effects that disturb normal cellular functions (Bucciantini, et al., 2002).

Similarly, models of autophagy deficiency often cause neurodegeneration or neurodegenerative phenotypes and an increase of autophagic capacity has potentially protective effects (reviewed in Rubinsztein, Mariño, \& Kroemer, 2011; Levine \& Kroemer, 2008). The effects of different disruptions of autophagy are summarized in Figure 18.

Autophagy plays an especially important role in neurons as these cells are long-living and have specialized structures, the synapses, which are often located a long distance from the nucleus where protein synthesis begins (Wang \& Hiesinger, 2012; see chapters 1.1). It has previously been suggested that defective proteostasis is a first indicator and possible common denominator of several neurodegenerative diseases (Douglas \& Dillin, 2010; Nixon, 2013; Liang \& Sigrist, 2018).

This theory builds on the fact that proteostasis is a key factor for cell survival. In addition to the cytotoxic effects of protein aggregates, cellular processes require functional proteins and organelles and can be perturbed depending on the severity of the imbalanced proteostasis (Douglas \& Dillin, 2010). Autophagy is the process which ensures the constant availability of functional organelles and proteins. Dysfunctional organelles, for example mitochondria with increased reactive oxygen species (ROS) production, and protein aggregates can be disposed of by the autophagic machinery (Okamoto \& Kondo-Okamoto, 2012; Menzies, Fleming, \& Rubinsztein, 2015).

Autophagy, however, becomes less effective with aging and is therefore thought to be a key factor for normal mammalian aging (Cuervo, et al., 2005; Martinez-Vicente, Sovak, \& Cuervo, 2005). In case of neurodegenerative diseases, the age-related decline of autophagic capacity is further aggravated by mutations that either cause an increase in misfolded proteins and consequently aggregation, dysfunctional or missing proteins that perturb organelle function like ATP production by mitochondria, thereby increasing the autophagic load, or by the disruption of autophagic proteins directly (Novack, 2017; Levine \& Kroemer, 2008). 
Taken together, the emerging role of autophagy in aging and neurodegeneration presents an interesting field of study with possible new therapeutic strategies for the treatment of neurodegenerative diseases. 


\subsection{Aims of the study}

Neurodegenerative diseases are a burden for the patients, their families and society in general. While our knowledge has greatly increased with the advancement of science, many questions remain unanswered. This thesis aims to look into the role of defective endocytosis as a possible cause for neurodegenerative diseases. Specifically, it will profit from the use of different mouse models for defective endocytosis, with a focus on endophilin deficient mice. These mice were characterized over the course of 18 months for motor deficits and biochemical as well as structural changes in the brain.

NGS analysis of endophilin mice has shown that the E3 ubiquitin ligase Fbxo32 is strongly up-regulated in the brains of these mice. As this protein has been proposed to be a mediator between UPS mediated protein degradation and autophagy, the scope of this study is also to explore the autophagic system in the endophilin mouse model. Perturbations were investigated using imaging and biochemical analysis.

In addition to this, a rescue experiment to revert the high levels of Fbxo32 was performed by crossing the endophilin mouse model with Fbxo32 deficient mice. The resulting offspring were then also characterized for motor behavior.

In short, the aims of the thesis were to:

- Characterize onset and progression of motor deficits in the endophilin mouse model and synaptojanin-1 and dynamin mutants

- Describe neurodegenerative changes in the brains of the endophilin mutants and correlate them with the observed phenotype

- Investigate the autophagic machinery in endophilin mutants as well as other endocytic mutants

- Genetically reduce Fbxo32 levels in endophilin mutants and describe these mice by motor behavior tests 


\section{Materials and Methods}

\subsection{Materials}

\subsubsection{Reagents and consumables}

All chemicals used for this study are listed in Table 1 . They were stored at room temperature (RT) or at $4^{\circ} \mathrm{C}$ following supplier's specifications. The consumables and kits used for this study are listed in Table 2. Consumables and Equipment used for cell culture experiments are listed in Table 3.

Table 1 - List of chemicals and their providers

\begin{tabular}{|l|l|l|}
\hline Chemical & Supplier & catalog number \\
\hline 2-Mercaptoethanol & Carl Roth & 4227.3 \\
\hline Acetic acid $\left(\mathrm{CH}_{3} \mathrm{COOH}\right)$ & Carl Roth & X895.2 \\
\hline Agarose & Biozym & 840004 \\
\hline Ammonium chloride $\mathrm{NH4Cl}$ & VWR & APLIA3260.0500 \\
\hline Ammonium Persulfate (APS) & AMRESCO & 0486 \\
\hline Bovine serum albumin (BSA) & Carl Roth & CP84.2 \\
\hline Bromphenolblue & Carl Roth & A512.1 \\
\hline Calcium chloride (CaCl 2$)$ & Carl Roth & CN93.1 \\
\hline Chloral hydrate & Sigma Aldrich & C8383 \\
\hline $\begin{array}{l}\text { Chrom(III)-Kaliumsulfat-Dodecahydrat } \\
\left.\text { (CrK(SO} 4)^{*}{ }^{*} 2 \mathrm{H}_{2} \mathrm{O}\right)\end{array}$ & Millipore & $7788-99-0$ \\
\hline Cold fish gelatin & Sigma Aldrich & G7765 \\
\hline DAPI & Carl Roth & 6335.1 \\
\hline Dithiothreitol (DTT) & Carl Roth & 6908.1 \\
\hline DNAse I Type II Bovine & Sigma Aldrich & d4527 \\
\hline
\end{tabular}




\begin{tabular}{|c|c|c|}
\hline Chemical & Supplier & catalog number \\
\hline EDTA & Carl Roth & 8043.2 \\
\hline Ethanol absolute & VWR & 20816,298 \\
\hline Ethidium bromide & VWR & E406 \\
\hline Finger paint (red) & Staedtler & 8801 \\
\hline Glycerol & Carl Roth & 7530.1 \\
\hline Glycin & Carl Roth & 3908.3 \\
\hline Goat Serum & Life Technologies & $10000 \mathrm{C}$ \\
\hline $\mathrm{HCl}$ & Sigma Aldrich & H1758 \\
\hline Heparin & Sigma Aldrich & H3393 \\
\hline HEPES & Carl Roth & HN77.2 \\
\hline Isoflurane & Sigma Aldrich & 792632 \\
\hline Isopentane & Sigma Aldrich & M32631 \\
\hline $\begin{array}{l}\text { Luminata }^{\mathrm{TM}} \text { Classico Western HRP } \\
\text { Substrate }\end{array}$ & Millipore & WBLUC0500 \\
\hline Luminata $^{\mathrm{TM}}$ Forte Western HRP Substrate & Millipore & WBLUF0500 \\
\hline Magnesium chloride $\left(\mathrm{MgCl}_{2}\right)$ & VWR & 8.14733 .0100 \\
\hline Magnesium sulfate $\left(\mathrm{MgSO}_{4}{ }^{*} 7 \mathrm{H}_{2} \mathrm{O}\right)$ & Sigma Aldrich & 63138 \\
\hline Methanol & VWR & 20903.368 \\
\hline Mowiol 4-88 & AppliChem & A9011 \\
\hline Optimum cutting temperature (OCT) & $\begin{array}{l}\text { Weckert } \\
\text { Labortechnik }\end{array}$ & 4583 \\
\hline Paraformaldehyde (PFA) & AppliChem & A3813 \\
\hline Entellan Neu mounting medium & Millipore & 107961 \\
\hline
\end{tabular}




\begin{tabular}{|c|c|c|}
\hline Chemical & Supplier & catalog number \\
\hline PhosStop tablets & Sigma Aldrich & 04906837001 \\
\hline Potassium chloride $(\mathrm{KCl})$ & Volu-Sol & 83608.26 \\
\hline $\begin{array}{l}\text { Potassium dihydrogen phosphate } \\
\left(\mathrm{KH}_{2} \mathrm{PO}_{4}\right)\end{array}$ & Carl Roth & 3904.1 \\
\hline Potassium phosphate dibasic $\left(\mathrm{K}_{2} \mathrm{HPO}_{4}\right)$ & Carl Roth & P749.2 \\
\hline $\begin{array}{l}\text { cOmplete ULTRA Tablets, EDTA-free, } \\
\text { Protease Inhibitor Cocktail }\end{array}$ & Sigma Aldrich & 05892953001 \\
\hline $\begin{array}{l}\text { Protease and Phosphatase Inhibitor } \\
\text { Cocktail (100X) }\end{array}$ & Thermo Fisher & 78440 \\
\hline ProteinaseK & Roche & 3115879001 \\
\hline Rotiphorese Gel 30 & Carl Roth & 3029.2 \\
\hline SDS & Sigma Aldrich & L4509-500G \\
\hline Skim Milk Powder & Fluka & 70166 \\
\hline Sodium bicarbonate $\left(\mathrm{NaHCO}_{3}\right)$ & Carl Roth & P029.3 \\
\hline Sodium chloride $(\mathrm{NaCl})$ & AppliChem & A1430,0010 \\
\hline Sodium hydroxide pelltets $(\mathrm{NaOH})$ & Carl Roth & 6771.1 \\
\hline $\begin{array}{l}\text { Sodium phosphate monobasic } \\
\left(\mathrm{NaH}_{2} \mathrm{PO}_{4}{ }^{*} \mathrm{H}_{2} \mathrm{O}\right)\end{array}$ & Carl Roth & K300.1 \\
\hline Sucrose & Carl Roth & 9097.1 \\
\hline TEMED & Sigma Aldrich & T7024 \\
\hline Tris-Base & Sigma Aldrich & T1503 \\
\hline Tris- $\mathrm{HCl}$ & Carl Roth & 9090.2 \\
\hline Triton $\mathrm{X}-100$ & AMRESCO & 0694 \\
\hline Tween-20 & AMRESCO & 0777-1L \\
\hline
\end{tabular}


Table 2 - Kits and consumable products

\begin{tabular}{|c|c|c|}
\hline Product & Supplier & Catalog number \\
\hline Amersham Hybond PO45 PVDF & GE Healthcare & 10600023 \\
\hline Coverslips $18 \times 18 \mathrm{~mm}$ & VWR & $631-1331$ \\
\hline Cuvettes polystyrol/polystyrene & Sarstedt & $67-742$ \\
\hline Embedding mold 22×22×20 & Thermo Scitific & 2219 \\
\hline Eppendorf tubes (1.5 mL) & StarLab & E1415-1500 \\
\hline Eppendorf tubes (2 mL) & StarLab & S1620-2700 \\
\hline Filter $\varnothing 150 \mathrm{~mm}$ & Machery-Nagel & 531015 \\
\hline GoTaq Flexi DNA polymerase kit & Promega & M8291 \\
\hline $\begin{array}{l}\text { High-Performance } \\
\text { chemiluminescence film }\end{array}$ & GE Healthcare & 28906837 \\
\hline Hydrophobic marker & Kisker Biotech & MKP-1 \\
\hline Medical X-Ray Film & Foma & ------ \\
\hline Microscope slides & Carl Roth & 2111 \\
\hline Needles 27G & B.Braun & 4657705 \\
\hline Nitrocellulose membrane & BIO-Rad & 1620115 \\
\hline $\begin{array}{l}\text { PageRuler Plus Prestained Protein } \\
\text { Ladder }\end{array}$ & Thermo Scientific & 26619 \\
\hline Pasteur pipet plastic & Carl Roth & EA63.1 \\
\hline $24 G$ perfusion needle & B.Braun & 4657675 \\
\hline Petri dish $2 \mathrm{~cm}$ & Sarstedt & 82.1135 .500 \\
\hline Petri dish $10 \mathrm{~cm}$ & Sarstedt & 82.1473 .001 \\
\hline Pierce BCA Protein Assay kit & Thermo Scientific & 23225 \\
\hline Plastic pipets $10 \mathrm{~mL}$ & Sarstedt & 86.1254 .025 \\
\hline
\end{tabular}




\begin{tabular}{|l|l|l|}
\hline Product & Supplier & Catalog number \\
\hline Rapid GolgiStain Kit & FD Neuroscience & PK401A \\
\hline Super frost slides & Th. Geyer & 7695019 \\
\hline Syringe $1 \mathrm{~mL}$ & VWR & $613-2040$ \\
\hline Tips $10 \mu \mathrm{L}$ TipOne & StarLab & $\mathrm{S} 111-3210$ \\
\hline Tips $20 \mu \mathrm{L}$ TipOne & StarLab & $\mathrm{S} 120-1810$ \\
\hline Tips $200 \mu \mathrm{L}$ TipOne & StarLab & $\mathrm{S} 1120-8800$ \\
\hline Tips $1000 \mu \mathrm{L}$ TipOne & StarLab & $\mathrm{S} 1111-6001$ \\
\hline Tubes $15 \mathrm{~mL}$ & Sarstedt & 62.554 .502 \\
\hline Tubes $50 \mathrm{~mL}$ & Corning & 430829 \\
\hline TUNEL kit & Sigma-Aldrich & 11684795910 \\
\hline Whatman Western Blot Paper & TH-Geyer & $4-01-60-0041$ \\
\hline
\end{tabular}


Table 3 - Cell Culture consumables and equipment

\begin{tabular}{|l|l|l|}
\hline Product & Supplier & Catalog number \\
\hline Centrifuge & Eppendorf & $5810 \mathrm{R}$ \\
\hline Dimethyl sulfoxide (DMSO) & Sigma-Aldrich & $41639-100 \mathrm{ML}$ \\
\hline DMEM, high glucose, pyruvate & ThermoFisher Scientific & 11995073 \\
\hline $\begin{array}{l}\text { Fetal Bovine Serum, qualified, } \\
\text { heat inactivated }\end{array}$ & ThermoFisher Scientific & 10500064 \\
\hline Incubator HERAcell 240 & ThermoFisher Scientific & 51026331 \\
\hline Neubauer counting chamber & VWR & $631-0696$ \\
\hline Nucleofactor X Core Unit & Lonza & AAT-1002B/X \\
\hline $\begin{array}{l}\text { P4 Primary Cell 4D-Nucleofector®8 } \\
\text { X Kit S (32 RCT) }\end{array}$ & Lonza & V4XP-4032 \\
\hline PBS tablets & VWR & $97062-732$ \\
\hline $\begin{array}{l}\text { Penicillin-Streptomycin (10,000 } \\
\text { U/mL) }\end{array}$ & ThermoFisher Scientific & 15140122 \\
\hline Petri dish 10 cm & Sarstedt & 83.3902 .300 \\
\hline T-25 flask & CytoOne & CC7682-4825 \\
\hline T-75 flask Paper & Sarstedt & 83.3911 .002 \\
\hline $\begin{array}{l}\text { Trypsin-EDTA (0.05\%), phenol } \\
\text { red }\end{array}$ & gibco & 11580626 \\
\hline Waterbath & Wemmert & \\
\hline
\end{tabular}




\subsubsection{Solutions}

All solutions and buffers used for this study are listed in Table 4 together with instructions how to prepare them.

Table 4 - List of solutions and buffers

\begin{tabular}{|c|c|}
\hline Solution & Recipe \\
\hline ammonium chloride & $50 \mathrm{mM} \mathrm{NH}_{4} \mathrm{Cl}$ in PBS \\
\hline $\begin{array}{l}\text { blocking solution for } \\
\text { immunocytochemistry }\end{array}$ & $\begin{array}{l}3 \%(\mathrm{w} / \mathrm{v}) \mathrm{BSA}, 0.2 \%(\mathrm{w} / \mathrm{v}) \text { cold fish gelatin, } 0.1 \% \\
(\mathrm{v} / \mathrm{v}) \text { Triton-X100 in PBS }\end{array}$ \\
\hline $\begin{array}{l}\text { blocking solution for } \\
\text { immunohistochemistry }\end{array}$ & $\begin{array}{l}3 \%(\mathrm{w} / \mathrm{v}) \mathrm{BSA}, 0.1 \%(\mathrm{w} / \mathrm{v}) \text { cold fish gelatin, } 0.1 \% \\
(\mathrm{v} / \mathrm{v}) \text { Triton-X100, } 0.05 \%(\mathrm{v} / \mathrm{v}) \text { Tween-20, } 1 \% \text { goat } \\
\text { serum in PBS }\end{array}$ \\
\hline Cell lysis buffer & $\begin{array}{l}1 \%(\mathrm{w} / \mathrm{v}) \mathrm{SDS}, 25 \mathrm{mM} \text { Tris Base, } 150 \mathrm{mM} \mathrm{NaCl}, 1 \\
\mathrm{mM} \text { EDTA, } 1 \%(\mathrm{v} / \mathrm{v}) \text { protease inhibitor cocktail in } \\
\mathrm{dH}_{2} \mathrm{O}\end{array}$ \\
\hline Chloral Hydrate & $4.125 \mathrm{mM}$ chloral hydrate in PBS \\
\hline Chromalaun-gelatin & $\begin{array}{l}0.3 \%(\mathrm{w} / \mathrm{v}) \text { gelatin, } 1 \mathrm{mM} \mathrm{CrK}\left(\mathrm{SO}_{4}\right)_{2}{ }^{*} 12 \mathrm{H}_{2} \mathrm{O} \text { in } \mathrm{dH}_{2} \mathrm{O} \text {; } \\
\text { heat } \mathrm{dH}_{2} \mathrm{O} \text { to } 58^{\circ} \mathrm{C} \text { and dissolve gelatin on the } \\
\text { stirrer, add the } \mathrm{CrK}\left(\mathrm{SO}_{4}\right)_{2}{ }^{*} 12 \mathrm{H}_{2} \mathrm{O} \text { only after the } \\
\text { solution has cooled down to } \mathrm{RT} \text {, filter }\end{array}$ \\
\hline HBSS buffer & $\begin{array}{l}5.36 \mathrm{mM} \mathrm{KCl}, 141.7 \mathrm{mM} \mathrm{NaCl}, 10 \mathrm{mM} \text { HEPES, } 3.4 \\
\mathrm{mM} \text { L-Giutamine, in } \mathrm{dH}_{2} \mathrm{O}\end{array}$ \\
\hline heparin solution & $100 \mathrm{U} / \mathrm{mL}$ in PBS \\
\hline Homogenization buffer & $\begin{array}{l}2 \mathrm{M} \mathrm{NaCl}, 0.5 \mathrm{M} \text { EDTA, } 1 \mathrm{M} \mathrm{HEPES} \text { in } \mathrm{dH}_{2} \mathrm{O} \mathrm{pH} 7.4 \\
\text { add } 1 \text { phospho-stop tablet/10 } \mathrm{mL}, 1 \text { protease } \\
\text { inhibitor tablet } / 50 \mathrm{~mL} \text { and } 1 \mathrm{mM} \text { DTT for activation }\end{array}$ \\
\hline $\begin{array}{l}\text { imaging buffer for live cell } \\
\text { imaging }\end{array}$ & $\begin{array}{l}136 \mathrm{mM} \mathrm{NaCl}, 2,5 \mathrm{mM} \mathrm{KCL}, 2 \mathrm{mM} \mathrm{CaCl}, 1 \mathrm{mM} \\
\mathrm{MgCl}_{2}, 10 \mathrm{mM} \text { HEPES, in } \mathrm{dH}_{2} \mathrm{O} \text {, p.H. } 7.4\end{array}$ \\
\hline
\end{tabular}




\begin{tabular}{|c|c|}
\hline Solution & Recipe \\
\hline 6x Laemmli buffer & $\begin{array}{l}62.5 \mathrm{mM} \text { Tris, } \mathrm{pH} 6.8,50 \%(\mathrm{v} / \mathrm{v}) \text { Glycerol, } 12 \%(\mathrm{w} / \mathrm{v}) \\
\text { SDS, } 0.06 \%(\mathrm{w} / \mathrm{v}) \text { Bromophenol blue. Add } 5 \% 2- \\
\text { Mercaptoetanol freshly prior to use }\end{array}$ \\
\hline $5 \%$ milk & $5 \%(\mathrm{w} / \mathrm{v})$ skim milk powder in TBS-T \\
\hline Mowiol 4-88 & $\begin{array}{l}\text { 3.6 M glycerol, } 3 \mathrm{M} \text { Mowiol } 4-88,133 \mathrm{mM} \text { Tris-Base } \\
\text { in } \mathrm{dH}_{2} \mathrm{O} \text {. Stir at } 45^{\circ} \mathrm{C} \text {, centrifuge at } 5000 \mathrm{~g} \text { for } 15 \mathrm{~min} \text {, } \\
\text { collect supernatant, store at }-20^{\circ} \mathrm{C}\end{array}$ \\
\hline $\begin{array}{l}\text { 4\% paraformaldehyde } \\
\text { (PFA) }\end{array}$ & $\begin{array}{l}4 \%(\mathrm{w} / \mathrm{v}) \mathrm{PFA} \text { in } \mathrm{PBS}, \mathrm{pH} 7.4 \text {; heat } \mathrm{PBS} \text { to } 58^{\circ} \mathrm{C} \text {, } \\
\text { add sodium hydroxide dropwise until PFA dissolves }\end{array}$ \\
\hline $\begin{array}{l}\text { phosphate buffered saline } \\
\text { (PBS) }\end{array}$ & $\begin{array}{l}137 \mathrm{nM} \mathrm{NaCl}, 2,7 \mathrm{mM} \mathrm{KCL}, 10 \mathrm{mM} \mathrm{Na}_{2} \mathrm{HPO}_{4} \times 2 \mathrm{H}_{2} \mathrm{O} \text {, } \\
2 \mathrm{mM} \mathrm{KH}_{2} \mathrm{PO}_{4} \text { in } \mathrm{dH}_{2} \mathrm{O} \text {, p.H. 7.4, autoclave }\end{array}$ \\
\hline Poly-L-Lysine & $1 \mathrm{mg} / \mathrm{mL}$ Poly-L-Lysine in $\mathrm{dH}_{2} \mathrm{O}$ \\
\hline $\begin{array}{l}\text { primary antibody solution for } \\
\text { immunocytochemistry }\end{array}$ & $3 \%(w / v)$ BSA, $0.2 \%(w / v)$ cold fish gelatin in PBS \\
\hline Running Buffer & $125 \mathrm{mM}$ Tris Base, $0.96 \mathrm{M}$ Glycine, $0.5 \%$ (w/v)SDS \\
\hline $\begin{array}{l}\text { secondary antibody solution } \\
\text { for immunocytochemistry }\end{array}$ & $\begin{array}{l}3 \%(\mathrm{w} / \mathrm{v}) \mathrm{BSA}, 0.2 \%(\mathrm{w} / \mathrm{v}) \text { cold fish gelatin, } 1 \%(\mathrm{v} / \mathrm{v}) \\
\text { goat serum in PBS }\end{array}$ \\
\hline SNET buffer & $\begin{array}{l}20 \mathrm{mM} \text { Tris (pH 8.0), } 5 \mathrm{mM} \text { EDTA (pH 8.0), } 400 \mathrm{mM} \\
\mathrm{NaCl}, 0.5 \%(\mathrm{w} / \mathrm{v}) \text { SDS in } \mathrm{dH}_{2} \mathrm{O}\end{array}$ \\
\hline TAE buffer & $\begin{array}{l}40 \mathrm{mM} \text { Tris, } 19 \mathrm{mM} \mathrm{CH}{ }_{3} \mathrm{COOH}, 1 \mathrm{mM} \text { EDTA, in } \\
\mathrm{dH}_{2} \mathrm{O}, \mathrm{pH} 8.0\end{array}$ \\
\hline TBS-T & $1 \%(v / v)$ Tween-20 in TBS \\
\hline TE buffer & $10 \mathrm{mM}$ Tris- $\mathrm{HCl}, 0.2 \mathrm{mM}$ EDTA in $\mathrm{dH}_{2} \mathrm{O}, \mathrm{pH} 7.5$ \\
\hline Transfer Buffer & $\begin{array}{l}25 \mathrm{mM} \text { Tris-Base, } 0.2 \mathrm{M} \text { Glycine, } 20 \%(\mathrm{v} / \mathrm{v}) \text { Methanol } \\
\text { in } \mathrm{dH}_{2} \mathrm{O}\end{array}$ \\
\hline Tris buffered saline (TBS) & $20 \mathrm{mM}$ Tris-Base, $150 \mathrm{mM} \mathrm{NaCl}$ in $\mathrm{dH}_{2} \mathrm{O}, \mathrm{pH} 7.6$ \\
\hline
\end{tabular}




\subsubsection{Antibodies}

All antibodies used for immunohistochemistry and western blot analysis are listed in Table 5 - Table 8.

Table 5 - Primary antibodies used for immunohistochemistry

\begin{tabular}{|l|l|l|l|}
\hline Target protein & Manufacturer & species & concentration \\
\hline a-synuclein & Cell Signaling 2628 & rabbit & $1: 100$ \\
\hline bassoon & Enzo SAP7F407 & mouse & $1: 400$ \\
\hline p-Foxo3A & Cell Signaling 13129 & rabbit & $1: 200$ \\
\hline Cleaved caspase 3 & Cell Signaling 9664 & rabbit & $1: 600$ \\
\hline GFAP & $\begin{array}{l}\text { Kind gift from Prof. K.-A. } \\
\text { Nave }\end{array}$ & rabbit & $1: 500$ \\
\hline
\end{tabular}

Table 6 - Secondary antibodies used for immunohistochemistry

\begin{tabular}{|l|l|l|l|l|}
\hline Target species & Fluorophore & Manufacturer & species & concentration \\
\hline Mouse & Alexa488 & Life Technologies & Goat & $1: 200$ \\
\hline Mouse & Alexa568 & Life Technologies & Goat & $1: 200$ \\
\hline Mouse & Alexa594 & Life Technologies & Goat & $1: 200$ \\
\hline Mouse & Alexa647 & Life Technologies & Goat & $1: 200$ \\
\hline Rabbit & Alexa488 & Life Technologies & Goat & $1: 200$ \\
\hline Rabbit & Alexa568 & Life Technologies & Goat & $1: 200$ \\
\hline Rabbit & Alexa594 & Life Technologies & Goat & $1: 200$ \\
\hline Rabbit & Alexa647 & Life Technologies & Goat & $1: 200$ \\
\hline
\end{tabular}


Table 7 - Primary antibodies used for western blot analysis

\begin{tabular}{|l|l|l|l|}
\hline Target protein & Manufacturer & species & concentration \\
\hline Actin & Millipore C4 & mouse & $1: 7500$ \\
\hline ATG5 & Cell Signaling 2630 & rabbit & $1: 1000$ \\
\hline ATG9A & Abcam 108338 & rabbit & $1: 1000$ \\
\hline ATG16L & Abcam 188642 & rabbit & $1: 1000$ \\
\hline Dynamin 1, 2 \& 3 & SySy 115002 & rabbit & $1: 1000$ \\
\hline Endophilin A1 & SySy 159 002 & rabbit & $1: 2000$ \\
\hline Endophilin A2 & SantaCruz A-11 & mouse & $1: 200$ \\
\hline Endophilin A3 & SantaCruz S-15 & goat & $1: 200$ \\
\hline Endophilin B1 & Novus Bio 24733 & mouse & $1: 200$ \\
\hline Fbxo32 & ECM AX2045 & rabbit & $1: 4000$ \\
\hline GAPDH & Sigma G9545 & rabbit & $1: 10000$ \\
\hline HPRT & Abcam 10479 & rabbit & $1: 4000$ \\
\hline $\begin{array}{l}\text { K48 linkage poly- } \\
\text { ubiquitin }\end{array}$ & Cell Signaling & rabbit & $1: 1000$ \\
\hline $\begin{array}{l}\text { K63 linkage poly- } \\
\text { ubiquitin }\end{array}$ & Cell Signaling & Rabbit & $1: 1000$ \\
\hline LC3 & Viva Bioscience VB2500 & mouse & $1: 5000$ \\
\hline Poly-ubiquitin & & & $1: 1000$ \\
\hline
\end{tabular}


Table 8 - Secondary antibodies used for western blot analysis

\begin{tabular}{|l|l|l|l|l|}
\hline Target species & Tag & Manufacturer & Species & concentration \\
\hline Rabbit & HRP & Cell Signaling & Goat & $1: 4000$ \\
\hline Rabbit & IR 680 & Li-Cor & Goat & $1: 7500$ \\
\hline Rabbit & IR 800 & Li-Cor & Goat & $1: 7500$ \\
\hline Mouse & IR 680 & Li-Cor & Goat & $1: 7500$ \\
\hline Mouse & IR 800 & Li-Cor & Goat & $1: 7500$ \\
\hline Goat & IR 800 & Li-Cor & Donkey & $1: 7500$ \\
\hline
\end{tabular}




\subsubsection{Plasmids}

The plasmids listed in Table 9 were used to transfect cells for live cell imaging.

Table 9 - Plasmid used in the study

\begin{tabular}{|l|l|l|l|}
\hline Plasmid name & vector & Protein & $\begin{array}{l}\text { Fluorescence } \\
\text { marker }\end{array}$ \\
\hline Endo2-GFP & pCMV_EGFP-N1 & endophilin A2 & EGFP \\
\hline CLC-EGFP & pCAG_EGFP & clathrin light chain & EGFP \\
\hline Fbxo32-mCherry & pCMV_mCherry-N1 & Fbxo32 & mCherry \\
\hline
\end{tabular}




\subsubsection{Equipment}

All equipment used for this thesis is listed in Table 10.

Table 10 - List of equipment

\begin{tabular}{|l|l|}
\hline Equipment & Manufacturer \\
\hline Accurpette & VWR \\
\hline Bio Photometer & Eppendorf \\
\hline Cryostat CM1850UV & Leica \\
\hline Curix 60 developer & AGFA \\
\hline DigiGait & Mouse specifics \\
\hline DMI 6000 b microscope & Leica \\
\hline EV231 power supply (genotyping) & Consort \\
\hline EW2200-2NM balance & Kern\&Sohn \\
\hline Examiner Z1 LSM710 microscope & Zeiss \\
\hline Fine balance ALJ 120-4 & Kern\&Sohn \\
\hline Grip strength meter & TSE systems \\
\hline Heating plate Mr3001 & Heidolph \\
\hline $37^{\circ} \mathrm{C}$ Incubator & memmert \\
\hline LKB perfusion pump & Phermacia \\
\hline LSM800 microscope & Zeiss \\
\hline Mastercycler epgradient & eppendorf \\
\hline MM400 Bead mill & Retsch \\
\hline NanoDrop Spectrophotometer & Peqlab \\
\hline Odyssey Infrared Imager & Li-Cor \\
\hline Perfection V850 Pro Scanner & Epson \\
\hline & \\
\hline
\end{tabular}




\begin{tabular}{|l|l|}
\hline Equipment & Manufacturer \\
\hline $\begin{array}{l}\text { Pipets }(2.5 \mu \mathrm{L}, 10 \mu \mathrm{L}, 20 \mu \mathrm{L}, 100 \mu \mathrm{L}, 200 \\
\mu \mathrm{L}, 1000 \mu \mathrm{L})\end{array}$ & eppendorf \\
\hline Power supply (SDS-PAGE) & Bio-Rad \\
\hline Rotarod & TSE systems \\
\hline SevenEasy pH Meter & Mettler Toledo \\
\hline Shaker Unimax 1010 & Heidolph \\
\hline Steam autoclave type DSL & Holzner \\
\hline Table centrifuge (5424) & eppendorf \\
\hline Thermomixer comfort & eppendorf \\
\hline Ultra View Spinning Disk & Nikon/Perkin Elmer \\
\hline UV imaging system & Intas \\
\hline Western blot system & Bio-Rad \\
\hline
\end{tabular}




\subsubsection{Software}

The software used for acquisition and analysis of data for this study is listed in Table 11.

Table 11 - List of software programs

\begin{tabular}{|l|l|l|l|}
\hline Software & Company & City/State & Country \\
\hline $\begin{array}{l}\text { Adobe Illustrator } \\
\text { CS6 }\end{array}$ & $\begin{array}{l}\text { Adobe Systems } \\
\text { Inc. }\end{array}$ & San Jose, CA & USA \\
\hline DigiGait Analyzer & Mouse Specifics & Framingham, MA & USA \\
\hline DigGait Imager & Mouse Specifics & Framingham, MA & USA \\
\hline Fiji & NIH & Bethesda, MD & USA \\
\hline Imaris 8.0.2 & Bitplane & Zurich & Switzerland \\
\hline Image Studio & Li-cor Bioscience & Bad Homburg & Germany \\
\hline $\begin{array}{l}\text { Leica Application } \\
\text { Suite (LAS) }\end{array}$ & $\begin{array}{l}\text { Leica } \\
\text { Microsystems } \\
\text { GmbH }\end{array}$ & Wetzlar & Germany \\
\hline Microsoft Office & $\begin{array}{l}\text { Microsoft } \\
\text { Corporation }\end{array}$ & Redmond, WA & USA \\
\hline Prism 6 & GraphPad & La Jolla, CA & USA \\
\hline Volocity & PerkinEImer & Rodgau & Germany \\
\hline ZEN black & Carl Zeiss AG & Oberkochen & Germany \\
\hline ZEN blue & Carl Zeiss AG & Oberkochen & Germany \\
\hline
\end{tabular}




\subsection{Methods}

\subsubsection{Experimental mice}

All animal procedures in this thesis were performed according to the European Guidelines for animal welfare (2010/63/EU) and with explicit permission from the Niedersächsisches Landesamt für Verbraucherschutz und Lebensmittelsicherheit (LAVES), registration number 14/1701. Animals were bred and housed in the Zentrale Tierexperimentelle Einrichtung (ZTE) Göttingen with ad libitum access to water and food. Animals were kept in groups of 1-5 animals on a $12 \mathrm{~h} / 12 \mathrm{~h}$ light/dark cycle. Experiments were conducted in the light phase. Individually ventilated or open cages were used. Endophilin mutant mice were originally described in (Milosevic, et al., 2011), synaptojanin-1 KO mice were described in (Cremona, et al., 1999), dynamin 1,3 DKO mice were described in (Raimondi, et al., 2011). Fbxo32 KO mice were kindly provided by Prof. Marco Sandri (University of Padova, Padova, Italy). All mouse lines were bred in the ZTE Göttingen. C57BL/6J WT animals were obtained from Janvier and used as an additional control throughout the thesis.

\subsubsection{DNA extraction and genotyping}

Mouse tail or ear punch samples were used for genotypic characterization of animals. All samples were digested in SNET buffer containing $167 \mu \mathrm{g} / \mathrm{mL}$ ProteinaseK, shaking for at least 1 hour at $55^{\circ} \mathrm{C}$. DNA was precipitated with $100 \%$ Ethanol and centrifuged at $20.000 \mathrm{~g}$ for $30 \mathrm{~min}$. The pellet washed with $70 \%$ Ethanol and centrifuged at 20.000 $\mathrm{g}$ for $20 \mathrm{~min}$. The final product was resuspended in TE buffer and DNA concentration was measured on a Nanodrop. Samples were stored at $-20^{\circ} \mathrm{C}$.

The GoTaq Flexi DNA polymerase kit from Promega was used to perform Polymerase-Chain-Reaction (PCR) to amplify WT and transgenic alleles for Fbxo32, endophilin $-1,-2,-3$, dynamin-1 or synaptojanin-1, yielding specific band sizes. The products were run on a $0.8 \%$ or $1.2 \%$ agarose gel. 
PCR protocol Fbxo32

Primer: $\quad$ MA61KO-F1 (AACAAAGAGACGGGGCAGCG)

MA61KO-R1 (TCGGCGAATGTCCTCACCTG)

MA61KO-LACZ (TGAGGGGACGACGACAGTATCG)

PCR reaction

\begin{tabular}{|c|c|}
\hline $5 x$ reaction buffer & $5 \mu \mathrm{l}$ \\
\hline $\mathrm{MgCl}_{2}(25 \mathrm{mM})$ & $2 \mu l$ \\
\hline dNTP (10mM) & $0.5 \mu \mathrm{l}$ \\
\hline Primer MA61KO-F1 $(10 \mu \mathrm{M})$ & $0.5 \mu \mathrm{l}$ \\
\hline Primer MA61KO-R1 (10 $\mu \mathrm{M})$ & $0.5 \mu \mathrm{l}$ \\
\hline Primer MA61KO-LACZ $(10 \mu \mathrm{M})$ & $0.5 \mu \mathrm{l}$ \\
\hline DMSO & $1.5 \mu \mathrm{l}$ \\
\hline Taq Polymerase $(5 \mathrm{U} / \mathrm{\mu l})$ & $0.25 \mu \mathrm{l}$ \\
\hline DNA ( 600 ng) & $3 \mu \mathrm{l}$ \\
\hline $\mathrm{H}_{2} \mathrm{O}$ & $11.25 \mu \mathrm{l}$ \\
\hline
\end{tabular}

\begin{tabular}{llll} 
PCR program & $95^{\circ} \mathrm{C}$ & $5 \mathrm{~min}$ \\
\cline { 2 - 3 } Fbxo32 & $95^{\circ} \mathrm{C}$ & $30 \mathrm{sec}$ & $\times 37$ \\
& $59,5^{\circ} \mathrm{C}$ & $45 \mathrm{sec}$ & \\
$72^{\circ} \mathrm{C}$ & $1,5 \mathrm{~min}$ & $7 \mathrm{~min}$ \\
& $72^{\circ} \mathrm{C}$ & $\infty$ &
\end{tabular}

WT band $\sim 422 \mathrm{bps}$, KO band $\sim 553 \mathrm{bps}$ 
PCR protocol Endophilin-A1

Primer: $\quad$ Endo1-for (CCACGAACGAACGACTCCCAC)

Endo1-RV1-WT (CGCACCTGCACGCGCCCTACC)

Endo1-RV3-Neo (TCATAGCCGAATAGCCTCTCC)

PCR reaction

\begin{tabular}{|l|l|}
\hline $5 x$ reaction buffer & $5 \mu \mathrm{l}$ \\
\hline $\mathrm{MgCl}_{2}(25 \mathrm{mM})$ & $2 \mu \mathrm{l}$ \\
\hline dNTP $(10 \mathrm{mM})$ & $0.5 \mu \mathrm{l}$ \\
\hline Primer Endo1-for $(10 \mu \mathrm{M})$ & $0.5 \mu \mathrm{l}$ \\
\hline Primer Endo1-RV1-WT $(10 \mu \mathrm{M})$ & $0.5 \mu \mathrm{l}$ \\
\hline Primer Endo1-RV3-Neo $(10 \mu \mathrm{M})$ & $0.5 \mu \mathrm{l}$ \\
\hline Betaine $(5 \mathrm{M})$ & $2 \mu \mathrm{l}$ \\
\hline Taq Polymerase $(5 \mathrm{U} / \mu \mathrm{l})$ & $0.2 \mu \mathrm{l}$ \\
\hline DNA ( 800 ng) & $3 \mu \mathrm{l}$ \\
\hline $\mathrm{H}_{2} \mathrm{O}$ & $10.8 \mu \mathrm{l}$ \\
\hline
\end{tabular}

\begin{tabular}{llll} 
PCR program & $94^{\circ} \mathrm{C}$ & $5 \mathrm{~min}$ \\
\cline { 2 - 3 } Endophilin-A1 & $94^{\circ} \mathrm{C}$ & $30 \mathrm{sec}$ & \\
& $60^{\circ} \mathrm{C}$ & $30 \mathrm{sec}$ & \\
& $72^{\circ} \mathrm{C}$ & $1.5 \mathrm{~min}$ \\
& $72^{\circ} \mathrm{C}$ & $5 \mathrm{~min}$ \\
& $10^{\circ} \mathrm{C}$ & $\infty$
\end{tabular}

WT band $\sim 384 \mathrm{bps}$, KO band $~ 950 \mathrm{bps}$ 
$\underline{\text { PCR protocol Endophilin-A2 }}$

Primer: Endo2-WT-For (CTTCTTGCCTTGCTGCCTTCCTTA)

Endo2-WT-Rev (GCCCCACAACCTTCTCGCTGAC)

Endo2-KO-For (CCTAGGGGCTTGGGTTGTGATGAGT)

Endo2-KO-Rev (CGTATGCAGCCGCCGCATTGCATC)

PCR reaction

\begin{tabular}{|l|l|}
\hline $5 \mathrm{x}$ reaction buffer & $5 \mu \mathrm{l}$ \\
\hline $\mathrm{MgCl}_{2}(25 \mathrm{mM})$ & $4 \mu \mathrm{l}$ \\
\hline dNTP $(10 \mathrm{mM})$ & $0.5 \mu \mathrm{l}$ \\
\hline Primer for $(10 \mu \mathrm{M}) \mathrm{WT} / \mathrm{KO}$ & $0.5 \mu \mathrm{l}$ \\
\hline Primer rev $(10 \mu \mathrm{M}) \mathrm{WT} / \mathrm{KO}$ & $0.5 \mu \mathrm{l}$ \\
\hline Betaine $(5 \mathrm{M})$ & $2 \mu \mathrm{l}$ \\
\hline Taq Polymerase $(5 \mathrm{U} / \mu \mathrm{l})$ & $0.25 \mu \mathrm{l}$ \\
\hline DNA ( 800 ng) & $3 \mu \mathrm{l}$ \\
\hline $\mathrm{H}_{2} \mathrm{O}$ & $9.25 \mu \mathrm{l}$ \\
\hline
\end{tabular}

\begin{tabular}{llll} 
PCR program & $95^{\circ} \mathrm{C}$ & $5 \mathrm{~min}$ \\
\cline { 2 - 3 } Endophilin-A2 & $95^{\circ} \mathrm{C}$ & $30 \mathrm{sec}$ & $\times 37$ \\
& $59.5^{\circ} \mathrm{C}$ & $45 \mathrm{sec}$ & \\
& $72^{\circ} \mathrm{C}$ & $1.5 \mathrm{~min}$ & $7 \mathrm{~min}$ \\
& $72^{\circ} \mathrm{C}$ & $\infty$ &
\end{tabular}

WT band $\sim 1280 \mathrm{bps}, \mathrm{KO}$ band $\sim 1000 \mathrm{bps}$ 
$\underline{\text { PCR protocol Endophilin-A3 }}$

Primer: mEndo3_SDL-F (CTCCCCATGGTGGAAAGGTCCATTC)

mEndo3_LOX-R (TGTGACAGTGGTGACCACAG)

mEndo3_KO-R (CAACGGACAGACGAGAGATTC)

PCR reaction

\begin{tabular}{|l|l|}
\hline $5 x$ reaction buffer & $5 \mu \mathrm{l}$ \\
\hline $\mathrm{MgCl}_{2}(25 \mathrm{mM})$ & $2 \mu \mathrm{l}$ \\
\hline dNTP $(10 \mathrm{mM})$ & $0.5 \mu \mathrm{l}$ \\
\hline Primer mEndo3_SDL-F $(10 \mu \mathrm{M})$ & $0.5 \mu \mathrm{l}$ \\
\hline Primer mEndo3_LOX-R $(10 \mu \mathrm{M})$ & $0.5 \mu \mathrm{l}$ \\
\hline Primer mEndo3_KO-R $(10 \mu \mathrm{M})$ & $0.5 \mu \mathrm{l}$ \\
\hline DMSO & $1.5 \mu \mathrm{l}$ \\
\hline Taq Polymerase $(5 \mathrm{U} / \mu \mathrm{l})$ & $0.2 \mu \mathrm{l}$ \\
\hline DNA ( 600 ng) & $3 \mu \mathrm{l}$ \\
\hline $\mathrm{H}_{2} \mathrm{O}$ & $11.8 \mu \mathrm{l}$ \\
\hline
\end{tabular}

\begin{tabular}{llll} 
PCR program & $95^{\circ} \mathrm{C}$ & $5 \mathrm{~min}$ \\
\cline { 2 - 3 } Endophilin-A3 & $95^{\circ} \mathrm{C}$ & $30 \mathrm{sec}$ & \\
& $58^{\circ} \mathrm{C}$ & $30 \mathrm{sec}$ & \\
& $72^{\circ} \mathrm{C}$ & $50 \mathrm{sec}$ \\
& $72^{\circ} \mathrm{C}$ & $10 \mathrm{~min}$ \\
& $10^{\circ} \mathrm{C}$ & $\infty$
\end{tabular}

WT band $\sim 325 \mathrm{bps}$, KO band $\sim 465 \mathrm{bps}$ 
PCR protocol Dynamin-1

Primer: $\quad$ DNW1-WT -fw (GCTCGGTGCTGGAGAATTTCGTGG)

DNW1-New KO -as (GGCTGCTCCGTAGAGGCTCACAGG)

DNW1-New KO -s (GTCAGTTTCATAGCCTGAAGAACG)

PCR reaction

\begin{tabular}{|c|c|}
\hline $5 x$ reaction buffer & $5 \mu \mathrm{l}$ \\
\hline $\mathrm{MgCl}_{2}(25 \mathrm{mM})$ & $2 \mu l$ \\
\hline dNTP (10mM) & $0.5 \mu \mathrm{l}$ \\
\hline Primer DNW1-WT -fw $(10 \mu \mathrm{M})$ & $0.8 \mu \mathrm{l}$ \\
\hline Primer DNW1-New KO -as $(10 \mu \mathrm{M})$ & $0.8 \mu \mathrm{l}$ \\
\hline Primer DNW1-New KO -s $(10 \mu \mathrm{M})$ & $0.8 \mu \mathrm{l}$ \\
\hline DMSO & $1.25 \mu \mathrm{l}$ \\
\hline Taq Polymerase $(5 \mathrm{U} / \mathrm{\mu l})$ & $0.2 \mu \mathrm{l}$ \\
\hline DNA ( 300 ng) & $3 \mu \mathrm{l}$ \\
\hline $\mathrm{H}_{2} \mathrm{O}$ & $10.65 \mu \mathrm{l}$ \\
\hline
\end{tabular}

\begin{tabular}{llll} 
PCR program & $95^{\circ} \mathrm{C}$ & $5 \mathrm{~min}$ \\
\cline { 2 - 3 } Dynamin-1 & $95^{\circ} \mathrm{C}$ & $30 \mathrm{sec}$ & \\
& $60^{\circ} \mathrm{C}$ & $40 \mathrm{sec}$ & \\
& $72^{\circ} \mathrm{C}$ & $1 \mathrm{~min}$ & \\
& $72^{\circ} \mathrm{C}$ & $7 \mathrm{~min}$ \\
& $10^{\circ} \mathrm{C}$ & $\infty$
\end{tabular}

WT band $\sim 600 \mathrm{bps}$, KO band $250 \mathrm{bps}$ 
PCR protocol Synaptojanin-1

Primer: NEO M2 (CGCTTCCTCGTGCTTTACGGTAT)

SJLA1 (GTCAGGTTACACTTGAACCAAC)

SJWTS1 (CCAATGCCAGTGAATTCCAGAC)

PCR reaction

\begin{tabular}{|l|l|}
\hline $5 \mathrm{x}$ reaction buffer & $5 \mu \mathrm{l}$ \\
\hline $\mathrm{MgCl}_{2}(25 \mathrm{mM})$ & $1 \mu \mathrm{l}$ \\
\hline dNTP $(10 \mathrm{mM})$ & $0.5 \mu \mathrm{l}$ \\
\hline Primer NEO M2 or Primer SJWTS1 $(10 \mu \mathrm{M})$ & $1 \mu \mathrm{l}$ \\
\hline Primer SJLA1 $(10 \mu \mathrm{M})$ & $1 \mu \mathrm{l}$ \\
\hline DMSO & $1.25 \mu \mathrm{l}$ \\
\hline Taq Polymerase $(5 \mathrm{U} / \mu \mathrm{l})$ & $0.2 \mu \mathrm{l}$ \\
\hline DNA ( 300 ng) & $3 \mu \mathrm{l}$ \\
\hline $\mathrm{H}_{2} \mathrm{O}$ & $12.05 \mu \mathrm{l}$ \\
\hline
\end{tabular}

\begin{tabular}{llll} 
PCR program & $95^{\circ} \mathrm{C}$ & $5 \mathrm{~min}$ \\
\cline { 2 - 3 } Synaptojanin-1 & $95^{\circ} \mathrm{C}$ & $30 \mathrm{sec}$ & \\
& $58.5^{\circ} \mathrm{C}$ & $30 \mathrm{sec}$ & \\
& $72^{\circ} \mathrm{C}$ & $1 \mathrm{~min}$ & \\
\cline { 2 - 3 } & $72^{\circ} \mathrm{C}$ & $7 \mathrm{~min}$ \\
& $10^{\circ} \mathrm{C}$ & $\infty$
\end{tabular}

WT band $\sim 580 \mathrm{bps}$, KO band $\sim 750 \mathrm{bps}$ 


\subsubsection{Motor behavior testing}

Behavior experiments were conducted with permission from the Niedersächsisches Landesamt für Verbraucherschutz und Lebensmittelsicherheit (LAVES) under the registration number $14 / 1701$. Mice were subjected to a battery of motor behavior test, namely grip strength test (GS), gait analysis (GAIT) and fixed-speed/accelerated Rotarod (FSRR/ARR). Experiments were always performed in this order, progressing from low to high difficulty for the animal to minimize effects from the previous experience. Experiments on endophilin mutants were performed at 8 time points between 1-18 months (specifically at 1, 2, 3, 6, 9, 12, 15 and 18 months of age). Endophilin mutants crossed with Fbxo32 $\mathrm{KO}$ mice were tested at 5 time points between 1-9 months (specifically at 1, 2, 3, 6 and 9 months of age). At least 10 mice were tested for each tested genotype.

On every experimental day, the health status of the animal was assessed by visual inspection and weight measurements. For all experiments, the average value was calculated for each genotype and age. Statistical analysis was performed with the statistics software GraphPad Prism 6. Outliers were excluded from the analysis if they differed more than $95 \%$ from the average values. Data were tested for normality and analyzed by ANOVA testing, corrected for multiple measurements by the Dunnett (for comparison of several groups with WT) or Tukey method (for comparison of several groups with each other) as specified in the results section.

\section{Grip strength test}

The protocol was adapted from (Mandillo, et al., 2008). A TSE grip strength meter equipped with a single bar grip was used to measure fore limb muscle strength in mice. Animals were hold by the tail and allowed to reflexively grab the metal bar. The mice were then pulled with constant force away from the bar and the maximum force, before the mice released the bar, was recorded. 15 measurements over 3 days (5 trials / day) were recorded to accommodate for the variability by the experimenter. The highest value for each day was normalized to the body weight and averaged over 3 days for analysis. 


\section{DigiGait}

Gait recording and analysis was performed using the DigiGait system (Mouse Specifics), described in (Amende, et al., 2005). For a detailed protocol, see (Rostosky \& Milosevic, 2018). One week before the first test, the DigiGait test chamber was prepared with Nutella and normal chow. Test animals were then placed into the covered chamber for 3 minutes to habituate to the environment and form a positive association. On the test day the paws of the test animal were painted using red finger paint in order to enhance the contrast of the paws to the brown fur of the endophilin mutants. When the paint was dried, the animals were placed into the DigiGait test chamber which was covered with a dark cloth. After a few minutes of accommodation

Table 12 - Examples of gait parameters measured by DigiGait.

\begin{tabular}{|c|c|}
\hline parameter & definition \\
\hline \multicolumn{2}{|l|}{ Gait rhythm } \\
\hline swing time & time the paw is in the air \\
\hline stance time & time the paw is in contact with the ground \\
\hline brake time & during stance, the time that the paw area is increasing \\
\hline propel time & during stance, the time that the paw area is decreasing \\
\hline norm. stance width & $\begin{array}{l}\text { distance between left and right fore or hind paws } \\
\text { normalized to animal width }\end{array}$ \\
\hline stance / swing & ratio of stance time to swing time \\
\hline paw drag & $\begin{array}{l}\text { area under the curve from full stance to paw lift-off; } \\
\text { increases if a paw is dragged behind }\end{array}$ \\
\hline \multicolumn{2}{|l|}{ Gait posture } \\
\hline limb loading & $\begin{array}{l}\text { maximal rate of change of paw area during brake phase, } \\
\text { determined as max } \mathrm{dA} / \mathrm{dt} \text {; indicator of how rapidly the } \\
\text { mouse decelerates }\end{array}$ \\
\hline Absolute paw angle & angle between the paw and the snout-tail axis \\
\hline Paw angle variability & standard deviation of the paw angle \\
\hline Shared stance & $\begin{array}{l}\text { percentage of hind limb stance time that both hind limbs } \\
\text { are in contact with the ground at the same time }\end{array}$ \\
\hline $\begin{array}{l}\text { Paw placement } \\
\text { positioning }\end{array}$ & $\begin{array}{l}\text { extent of overlap between ipsilateral fore and hind paws } \\
\text { during full stance }\end{array}$ \\
\hline
\end{tabular}


time, the treadmill was started and the running recorded with a high-speed camera under the transparent treadmill. Videos were recorded at $10 \mathrm{~cm} / \mathrm{s}, 20 \mathrm{~cm} / \mathrm{s}$ and 30 $\mathrm{cm} / \mathrm{s}$ running speed. Animals that did not run at the maximum speed or refused running all together, were tested on the next day and if necessary the day after that. If they did not run at three independent test days, animals were excluded from this test. Acquired videos were analyzed using the DigiGait Analysis software that extracts "digital paw prints" from the videos and measures gait parameters based on the time the paws are in contact with the treadmill and the relative positions of the paws towards each other. The maximum speed the animals were able to run as well as $\sim 50$ parameters calculated from the Analysis software were considered for analysis (s. Table 12).

\section{Rotarod}

The Rotarod test was used to evaluate motor coordination and balancing in experimental animals. The procedure was adapted from (Wegener, et al., 2014). In this test, animals were placed on a $3 \mathrm{~cm}$ diameter rubber rod, ca. $8 \mathrm{~cm}$ above the ground, which rotated at a fixed or accelerating speed. Fall latency was measured with a light beam at the bottom of the Rotarod apparatus. Animals were trained for 2 days ( 2 trials / day) at the low rotation speed of 5 rotations per minute (rpm) for $180 \mathrm{~s}$ / trial. In this phase, animals were replaced on the rod if they fell down. In the testing phase, the rod was accelerated from 5-40 rpm over $180 \mathrm{~s}$. Every animal underwent 4 trials (2 / day) and the fall latency was measured for each trial.

\subsubsection{Cell culture procedures}

The cell culture experiments reported in this thesis were performed in mouse embryonic fibroblasts (MEFs) that carried a conditional KO for dynamin 1, 2 and 3.

\section{Basic Cell Culture}

\section{Culture conditions for MEFs}

MEFs were cultured in $10 \mathrm{~cm}$ petri dishes or T-75 flasks in Dulbecco's Modified Eagle Medium (DMEM) with 10\% fetal bovine serum and 1\% Penicillin/Streptomycin 
cocktail, henceforth growth medium. Cell were handled under a sterile hood and incubated at $37^{\circ} \mathrm{C}$ and $5 \% \mathrm{CO}_{2}$ in an eppendorf incubator.

\section{Splitting cells}

Cultured cells were split every 2-3 days. Cells were washed with sterile phosphatebuffered saline (PBS) pre-heated to $37^{\circ} \mathrm{C}$ and incubated with trypsin enzyme for 3 $\min$ at $37^{\circ} \mathrm{C}$. Detachment of the cells was controlled on a light microscope. The enzymatic reaction was stopped with growth medium and the cells collected in a 50 $\mathrm{ml}$ Falcon tube. Cells were spun down at 1,000 rpm for $3 \mathrm{~min}$ and re-suspended in fresh growth medium. Cells were diluted in a 1:4 to 1:5 ratio with growth medium in a new petri dish.

\section{Cryopreservation and thawing of MEFs}

To freeze cells, cells were washed with $37^{\circ} \mathrm{C}$ warm PBS, detached from the petri dish with $3 \mathrm{~min}$ incubation of trypsin at $37^{\circ} \mathrm{C}$ and re-suspended in growth medium. They were then spun down at $1,000 \mathrm{~g}$ for $3 \mathrm{~min}$ and re-suspended in $375 \mu \mathrm{L}$ DMEM. To this cell suspension, $375 \mu \mathrm{L}$ freezing medium containing 10\% FBS $+10 \%$ DMSO, were carefully added and gently mixed. The cells were divided into 3 previously labeled cryotubes which were stored in a plastic container filled with isopropanol in which cells were slowly cooled down in a $-80^{\circ} \mathrm{C}$ freezer $\mathrm{O} / \mathrm{N}$. For long-term storage, cryotubes were submerged in a liquid nitrogen tank. To defrost MEFs, cells were thawed in a $37^{\circ} \mathrm{C}$ waterbath until all ice crystals were dissolved. The cells with the freezing medium were plated in $10 \mathrm{~mL}$ growth medium and incubated at $37^{\circ} \mathrm{C}, 5 \%$ $\mathrm{CO}_{2} \mathrm{O} / \mathrm{N}$. The next day, the medium was replaced with fresh growth medium.

\section{Preparation of MEFs for biochemical analysis}

MEFs were collected after washing the cells with pre-heated PBS by trypsin treatment of the culture dish for $3 \mathrm{~min}$ at $37^{\circ} \mathrm{C}$. The cells were re-suspended in growth medium to stop the enzymatic reaction and spun down at $1,000 \mathrm{~g}$ for $3 \mathrm{~min}$. The cells were resuspended in $1 \mathrm{~mL}$ warm PBS and collected in a $2 \mathrm{~mL}$ tube. Cells were centrifuged again at 1,000 $\mathrm{g}$ for $3 \mathrm{~min}$ and the pellet lysed in $200 \mu \mathrm{L}$ cell lysis buffer (s. Table 4) for $30 \mathrm{~min}$ on a $4^{\circ} \mathrm{C}$ shaker. Lysed cells were passed through a $27 \mathrm{G}$ syringe 20-30 times and the samples further handled as detailed in 2.2.7. 


\section{Induction of triple knock-out in dynamin cTKO MEFs}

MEFs carrying a conditional TKO (cTKO) for dynamin 1,2 and 3 as originally described in (Ferguson, et al., 2009; Park, et al., 2013) were kindly provided by Pietro De Camilli (Yale University). The cells behave like WT MEFs until the conditional KO is activated by $4-H y d r o x y t a m o x i f e n(\mathrm{OHT})$ treatment. Before the induction of the TKO, cells were cultured for at least 2 passages under standard conditions. The cells were then split as described above except that in the final cultivation $30 \mathrm{mM}$ OHT dissolved in pure ethanol was added to the growth medium, resulting in a final concentration of $3 \mu \mathrm{M} \mathrm{OHT}$ in the petri dish. Control cells were treated with equal amounts of pure ethanol without OHT. After $24 \mathrm{~h}$, the $\mathrm{OHT}$ or ethanol was renewed and after $48 \mathrm{~h}$ the medium changed to normal growth medium leaving $5-10 \%$ of the old medium, resulting in a concentration of $100-300 \mathrm{nM}$ of $\mathrm{OHT}$ to prevent potentially non-induced cells from proliferating. Complete TKO of dynamin is established 5 days after the first treatment with $\mathrm{OHT}$. At this stage, the cells no longer proliferate, for that reason the cells have to be used between day 5 and day 10 before the cells start dying. The efficiency of the KO was confirmed by western blot for a pan-dynamin antibody on the day the cells were used for experiments. Since the TKO cells no longer proliferate, the medium was changed every other day without splitting the cells. Ethanol treated control cells were split every 2 days to keep an equal cell density.

\section{Live imaging of transfected MEFs}

\section{Poly-L-Lysine plate preparation}

Coverslips for live cell imaging were prepared by washing them with $1 \mathrm{M} \mathrm{HCl}$ for at least $4 \mathrm{~h}$ on a shaker. The coverslips were then quickly rinsed with distilled $\mathrm{H}_{2} \mathrm{O}$ and washed for 20 min in distilled $\mathrm{H}_{2} \mathrm{O}$ on a shaker. After that the coverslips were quickly rinsed twice with $96 \%$ ethanol and then washed with absolute ethanol for 20-60 min and then stored in fresh absolute ethanol until further use. Before the use of the coverslips for cell culture they were coated with Poly-L-Lysine (PLL) to increase the attachment of the cells. For this, coverslips were quickly flamed with a Bunsen burner and inserted into a 12-well plate. $500 \mu \mathrm{L}$ of $1 \mathrm{mg} / \mathrm{mL} \mathrm{PLL}$ in double-distilled $\mathrm{H}_{2} \mathrm{O}$ was incubated for at least $30 \mathrm{~min}$ at $37^{\circ} \mathrm{C}$ on the coverslips. After this, coverslips were washed twice with double-distilled $\mathrm{H}_{2} \mathrm{O}$ and dried under the sterile hood for about 20 
min. 12-well plates were stored under sterile conditions at $4^{\circ} \mathrm{C}$ for a maximum of 1 month before use.

\section{Amaxa 4D-Nucleofector transfection}

Expression of fluorescence-tagged proteins in cells was achieved through transfection of MEFs with plasmid DNA carrying the desired DNA sequence. The Amaxa 4D-Nucleofector kit from Lonza was used following manufacturer's instructions. Transfection reagent was prepared by mixing $16.4 \mu \mathrm{L}$ Nucleofactor solution with $3.6 \mu \mathrm{L}$ supplement per reaction. Cells were washed, detached from the petri dish with $3 \mathrm{~min}$ incubation with trypsin at $37^{\circ} \mathrm{C}$ and re-suspended in growth medium. After spin-down for $3 \mathrm{~min}$ at $1,000 \mathrm{~g}$, cells were re-suspended in $4 \mathrm{~mL}$ PBS and $10 \mu \mathrm{L}$ inserted into a Neubauer counting chamber. All cells in the 4 big squares were counted and the concentration in cells / $\mathrm{mL}$ calculated by the average number of cells / square $\times 10,000$. From the cell suspension, 200,000 cells were used per reaction. The appropriate volume of the cell suspension was spun down for $3 \mathrm{~min}$ at $1,000 \mathrm{~g}$ and the cell pellet re-suspended in $20 \mu \mathrm{L}$ transfection reagent in a $1.5 \mathrm{~mL}$ tube. 0.5-1 $\mu \mathrm{g}$ Plasmid DNA were gently mixed to the cells and the suspension transferred to small Amaxa cuvettes while avoiding introducing air bubbles. The cuvettes were placed in a Nucleofactor X Unit and subjected to the program CZ-167 for MEFs. After transfection, cells were allowed to rest for $10 \mathrm{~min}$ before they were re-suspended in growth medium. Each reaction was split on 2 wells and plated in 2 $\mathrm{mL}$ growth medium / well on a 12-well plate with PLL-coated coverslips. Plasmid DNA was expressed for $36 \mathrm{~h}$ before cells were imaged on a spinning disk microscope or fixed for imaging on a LSM microscope.

\section{Live cell imaging}

Transfected MEFs were imaged on a Nikon Perkin Elmer spinning disk microscope. Coverslips on which the cells were grown were quickly washed in warm PBS and then inserted into an imaging chamber which was then filled with imaging buffer. Live cells were imaged in a chamber heated to $37^{\circ} \mathrm{C}$ with a $63 x$ or $100 x$ objective. Movies were recorded with Velocity software for 4-10 min with 15 frames / min. 


\subsubsection{Brain dissection from young and adult mice}

Tissue from living animals was collected for biochemical and histochemical analysis to demonstrate the in vivo relevance of the endophilin mouse model.

\section{Dissection of whole brain ( $\mathrm{p} 0)$}

Newborn animals were collected a few hours after birth and kept on a warm plate for a maximum of $1 \mathrm{~h}$ before dissection. The pups were picked up and the head quickly decapitated with big scissors. Small scissors were inserted in the foramen magnum (the hole in the skull through which the spinal cord passes) and a cut made towards the eye and from there towards the skull midline on both sides so that the upper part of the skull could be removed. The whole brain was removed with a spatula and washed in PBS. For biochemical analysis, the brain was then stored in an $1.5 \mathrm{~mL}$ tube, quick-frozen in liquid nitrogen and stored at $-80^{\circ} \mathrm{C}$ until further use.

\section{Dissection of p14 or adult mice brains}

Juvenile and adult brains were dissected into cortices, hippocampi, cerebellum and rest of the brain. To this end, animals were anesthetized with isoflurane and euthanized by cervical dislocation. The mice were then decapitated and the brain extracted as detailed above and placed in a small petri dish containing PBS with the cranial side facing down. The connections between cortices and thalamus were cut with a small scalpel. The brain was then turned so that the cranial side faced up again. Holding the brain in the midbrain between cortices and cerebellum with forceps, a cut was made along the midline between the 2 cortices so that they were detached from the brain stem. From each cortex, the hippocampus was removed by using a small spatula to unfold the hippocampus and a scalpel to separate it from the cortex. The cerebellum was detached from the brain stem with one sagittal and one transverse cut. All samples were stored in $1.5 \mathrm{~mL}$ tubes and quick-frozen in liquid nitrogen and stored at $-80^{\circ} \mathrm{C}$ until further processing. 


\subsubsection{Staining procedures on brain slices and cells}

\section{Preparation of brain slices for immunostainings}

Brain slices were obtained from juvenile and aged animals by perfusion and from $\mathrm{p0}$ animals by brain fixation. Brains were then frozen in optimum cutting temperature (OCT) solution and cut on a cryostat.

\section{Perfusion}

The perfusion protocol was modified from (Gage, Kipke, \& Shain, 2012). Animals were weighed and anesthetized through intraperitoneal injection of $400 \mu \mathrm{g}$ chloral hydrate (in PBS) / g body weight. When the animal was unresponsive to pinching (after 10-15 min), the mouse was fixed on a styrofoam dish and the abdomen and thorax cut open to reveal the heart. A 25G5/8 perfusion needle, bend in a $\sim 80^{\circ}$ angle and attached to a peristaltic pump, was inserted into the left ventricle of the heart and a small cut made at the right atrium. $9 \mathrm{~mL}$ cold PBS / min were pumped through the heart for 3 min to wash out the blood from the entire body. After that, cold $4 \%$ PFA was pumped at $10 \mathrm{~mL}$ per min to pre-fix the tissue immediately. After $\sim 7 \mathrm{~min}$, the brain was dissected from the animal and post-fixed in cold 4\% PFA over night $(\mathrm{O} / \mathrm{N})$.

\section{Brain fixation of p0 brains}

Brains from new-born pups were collected in the morning, a few hours after birth. Animals were killed by decapitation and the whole brain dissected and washed in PBS. Brains were then fixed in cold 4\% PFA for $3 \mathrm{~h}$.

\section{Freezing and cryostat cutting}

After fixation, brains were washed 3 times for 5 min with PBS and then dehydrated with increased concentration of sucrose $(12 \%, 15 \%, 18 \%$ sucrose in PBS for $8-12 \mathrm{~h}$ each). Brains were then stored in a cryomold and immersed in OCT solution. The mold was then quick frozen in isopentane cooled down by liquid nitrogene. Samples were stored at $-80^{\circ} \mathrm{C}$ until further use. Sagittal sections were cut on a cryostat set to $-20^{\circ} \mathrm{C}$. The frozen OCT blocks were attached to the cryostat cutting block and then cut at $10 \mu \mathrm{m}$ thickness and collected on Super Frost Plus slides. Slides were stored at $-20^{\circ} \mathrm{C}$. 


\section{Immunostaining on cryosections}

Immunohistochemical stainings were performed on frozen brain sections. The protocol was modified from (Evilsizor, Ray-Jones, Lifshitz, \& Ziebell, 2015). Sections were encircled with a hydrophobic marker and re-hydrated with PBS. $50 \mathrm{mM}$ ammonium chloride was used to quench fluorescence after PFA treatment and washed with PBS. Samples were blocked with blocking solution for $1 \mathrm{~h}$ at room temperature (RT). The primary antibody was diluted according to producers' specifications in blocking solution and incubated on the section at $4^{\circ} \mathrm{C} O / N$. The next day, sections were washed 3 times for 10 min with PBS and incubated with a fluorescence-tagged secondary antibody diluted in blocking solution for $1 \mathrm{~h}$ at RT. After washing with PBS, nuclei were stained for 5 min with DAPI solution and mounted with Mowiol 4-88. Sections were dried for $3 \mathrm{~h}$ at $37^{\circ} \mathrm{C}$ and then stored at $4^{\circ} \mathrm{C}$. Sections were imaged within 1 week after staining on a laser scanning microscope (LSM), LSM710 or LSM800, using ZEN blue or ZEN black software respectively.

\section{Immunostaining on cultured cells}

For immunocytochemical stainings, cells were grown on $18 \mathrm{~mm}$ diameter coverslips. As adapted from (Marchenko \& Flanagan, 2007), cells were washed in PBS and fixed for $1 \mathrm{~h}$ at RT with $4 \%$ PFA. Cells were quenched with $50 \mathrm{mM}$ ammonium chloride for 20 min at RT and then blocked with blocking solution. Primary antibody was diluted according to manufacturer's specifications in blocking solution without Triton-X100 and incubated on the cells $\mathrm{O} / \mathrm{N}$ at $4^{\circ} \mathrm{C}$. On the following day, the cells were washed 3 times for 10 min each in PBS and then incubated with a fluorescence-tagged secondary antibody diluted in secondary antibody solution. Cells were then washed 2 times for 10 min with PBS and counter-stained with DAPI solution for 2 min. After final washing in PBS for 15 min, the coverslips were mounted with Mowiol 4-88, dried for $3 \mathrm{~h}$ at $37^{\circ} \mathrm{C}$ and then stored at $4^{\circ} \mathrm{C}$. Coverslips were imaged within 1 week after staining on a LSM710 or LSM800 using ZEN blue or ZEN black software respectively.

\section{TdT-mediated dUTP-biotin nick end labeling (TUNEL) assay}

Apoptotic and necrotic cell were identified using the "In Situ Cell Death Detection Kit, Fluorescein" from Sigma-Aldrich according to manufacturer's instructions. In short, 12 
brain cryosections were selected ( 9 test samples, 1 positive control and 2 negative controls), encircled with a hydrophobic marker and briefly washed with PBS. Sections were then post-fixed with 4\% PFA for 20 min at RT, briefly washed 2 times with PBS and then washed for 30 min with PBS. Sections were permeabilized on ice with $0.1 \%$ Triton X-100 (in water) and washed 2 times for 2 min with PBS. The positive control was then incubated with $833 \mathrm{U} / \mathrm{mL}$ DNAse I in HBSS buffer for 10 min at RT (the other sections were washed in PBS in that time). TUNEL reaction mix was incubated on the samples and positive control for $60 \mathrm{~min}$ at $37^{\circ} \mathrm{C}$ in a wet chamber, while the negative controls were incubated under the same conditions with label solution only (no enzyme). Sections were washed for 5 min with PBS, counter-stained with DAPI and washed again 2 times for 5 min before mounting with Mowiol 4-88. Samples were stored at $4^{\circ} \mathrm{C}$ for a maximum of 3 days before imaging on a LSM800 using ZEN black software (TUNEL channel: exitation 450-500nm, detection 515-565nm).

\section{Golgi staining on mouse brains}

Golgi staining is a well-established method to analyze neuron morphology in brains that was first used already in 1873 by Camillo Golgi (Ramón-Moliner, 1970). This silver staining randomly stains single cells in nervous tissue and thus allows analyzing cell morphology in this densely packed tissue.

\section{Tissue Preparation}

Golgi staining was performed with the Rapid GolgiStain Kit from FD Neuroscience according to manufacturer's instructions. Impregnation solution was prepared with equal amounts Solution A and B $24 \mathrm{~h}$ before the staining and kept in the dark. Experimental animals were anesthetized with isoflurane and euthanized by cervical dislocation. The whole brain was dissected as described in section 2.2.5. The brain was quickly rinsed with double-distilled $\mathrm{H}_{2} \mathrm{O}$ and then immersed in $4 \mathrm{~mL}$ impregnation solution in a small glass bottle. The solution was renewed the next day and the brain kept for a total of 14 days while staying in the dark. After this, the solution was replaced with Solution C and incubated for $72 \mathrm{~h}$. Solution C was renewed after $24 \mathrm{~h}$.

\section{Preparation of gelatin-coated slides}

Brain sections for Golgi staining were collected on gelatin-coated slides to increase attachment forces. Glass slides were placed in a slide holder and washed with hot 
soapy water for $10 \mathrm{~min}$. They were then washed for $30 \mathrm{~min}$ under a running water tab. Slides were washed with distilled water for $10 \mathrm{~min}$ and then dipped twice for $10 \mathrm{~s}$ each in chromalaun solution (s. Table 4). Slides were dried and stored in dust free boxes until further use.

\section{Cryostat sectioning of Golgi staining}

Brains were frozen in isopentane pre-cooled on dry ice. The samples were slowly immersed in isopentane and kept there for $1 \mathrm{~min}$ until the tissue was completely frozen. Brains were mounted on a cryostat sample holder with OCT and cooled on dry ice for $10 \mathrm{~min}$. The sample holder was then transferred into the cryostat chamber and allowed to adjust for $5 \mathrm{~min}$. Sagittal sections were cut at 100, 150 or $200 \mu \mathrm{m}$ thickness and then attached to gelatin-coated slides with 1 drop of Solution $\mathrm{C}$ of the Rapid GolgiStain Kit. Slides were dried at RT O/N in the dark and stained on the next day.

\section{Golgi staining procedure}

Samples were washed in double-distilled $\mathrm{H}_{2} \mathrm{O}$ for 2 times 4 min. The silver staining was performed with a freshly prepared staining solution consisting of 1 part Solution D, 1 part Solution E of the Rapid GolgiStain Kit and 2 parts double-distilled $\mathrm{H}_{2} \mathrm{O}$ for 10 minutes with frequent stirring. Sections were washed again for 2 times 4 min with new double-distilled $\mathrm{H}_{2} \mathrm{O}$. Samples were de-hydrated with increasing ethanol concentration $(50 \%, 70 \%, 96 \%, 100 \%, 100 \%, 100 \%, 100 \%)$ for 4 min each. The sections were cleared with xylene 3 times for 4 min and mounted with Entellan New. Golgi-stained sections were dried $\mathrm{O} / \mathrm{N}$ in the dark and then stored at $4^{\circ} \mathrm{C}$. Imaging was performed with a Leica DMI $6000 \mathrm{~b}$ microscope operated with LAS software and using a 100x dry objective. Access to the microscope was kindly granted by Prof. Dr. Tiago Outeiro. Analysis of spine density and shape was performed using the Imaris filament tracer (Figure 19). 


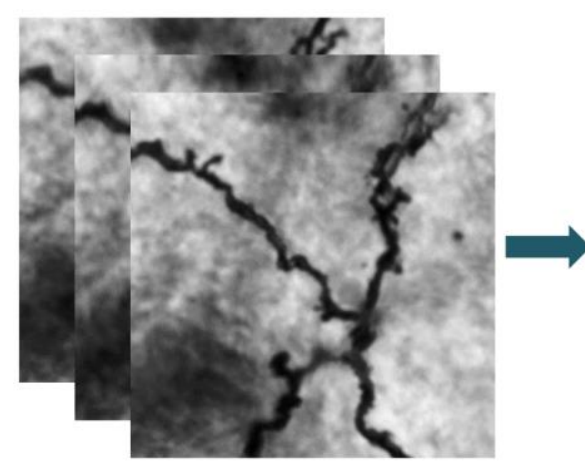

z-stacks

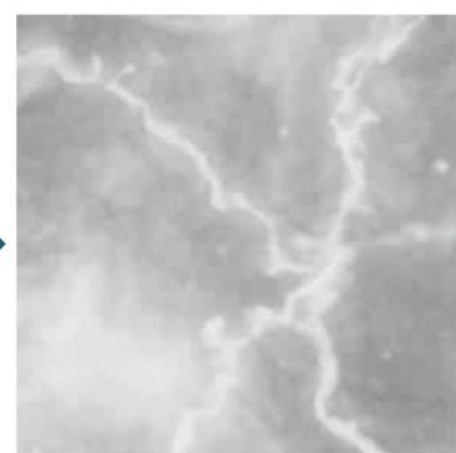

maximum projection

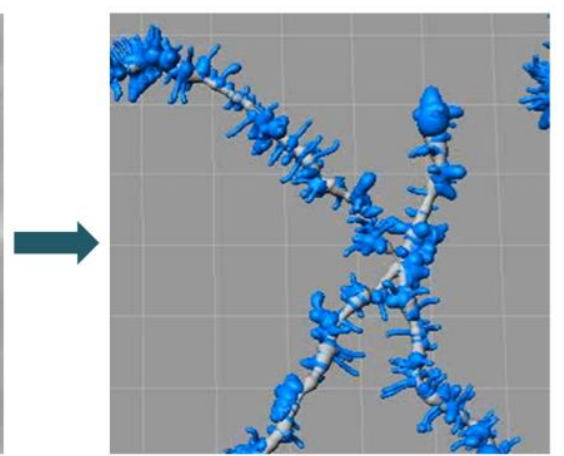

Imaris filament tracer

Figure 19 - Principle of Imaris filament tracer. Z-stacks from Golgi stained brain sections were projected in Imaris to trace dendrites in a semi-automated manner. Spines were identified and classified with the Imaris spine analyzer.

\subsubsection{Biochemical analysis}

Full brains ( $\mathrm{p} 0$ animals) or cortex samples ( $\mathrm{p} 14$ and older animals) were used for analysis of protein content in the test animals. In short, brains were homogenized, protein content quantified, run on an SDS-PAGE and proteins identified by western blot and immunological staining. The protocol is described in (Murdoch, et al., 2016).

\section{Brain/cortex homogenates}

Samples were removed from the $-80^{\circ} \mathrm{C}$ freezer and immediately put in a $2 \mathrm{~mL}$ tube containing $1080 \mu \mathrm{L}$ activated homogenization buffer (containing protease inhibitor and phosphatase inhibitor) and 3 metallic beads of $\sim 4 \mathrm{~mm}$ diameter. Tubes were oscillated on a bead mill for $1 \mathrm{~min}(20 \mathrm{~Hz}$ for $\mathrm{p} 0$ brains, $30 \mathrm{~Hz}$ for other samples). Tubes were spun down with a centrifuge set to $11,000 \mathrm{~g}$ for $10 \mathrm{~s}$ and $120 \mu \mathrm{L} 20 \%$ SDS was added to the tube. The oscillation - spin-down cycle was repeated a second time (without adding further SDS) and a third or fourth time if necessary (e.g. if tissue parts were still visible). Finally, the homogenates were mixed by passing 15-30 times through a $27 \mathrm{G}$ needle attached to a $1 \mathrm{~mL}$ syringe.

\section{Pierce BCA Protein Assay for protein quantification}

Protein content was quantified using the Pierce BCA Protein Assay kit (Bio-Rad) according to manufacturer's instructions. Homogenates were diluted 1:40 in distilled $\mathrm{H}_{2} \mathrm{O}$ and $50 \mu \mathrm{L}$ of the sample (in duplicate) or standard (with known concentrations of 
albumin protein) added to $1 \mathrm{~mL} \mathrm{BCA}$ working solution (consisting of 50 parts reagent A : 1 part reagent $B$ ). The mixture was incubated for 30 min at $37^{\circ} \mathrm{C}$ in the dark and the absorbance at $562 \mathrm{~nm}$ measured with a spectrophotometer. Protein content was calculated based on the absorbance measured in the known standards with Microsoft Excel 2013 software. Brain homogenates were adjusted to a protein concentration of $50 \mu \mathrm{g}$ in $12 \mu \mathrm{L}$ 6x Laemmli buffer diluted 1:6 in distilled $\mathrm{H}_{2} \mathrm{O}$.

\section{SDS-PAGE (sodium dodecyl sulfate polyacrylamide gel electrophoresis)}

SDS-PAGE was used to separate denatured proteins based on their molecular weight. Polyacrylamid gels were cast with the gel preparation system from Bio-Rad. The resolving gel, in which the proteins are separated, was made at a concentration of $10 \%, 12 \%$ or $15 \%$ polyacrylamide (see Table 13 ) depending on the molecular weight of the proteins of interest. High-concentration gels were preferably used for a good resolution of proteins with small molecular weight and low-concentration gels for proteins with high molecular weight. The gel was mixed in a $50 \mathrm{~mL}$ tube and then poured between the 2 glass plates of the gel preparation system and $\sim 500 \mu \mathrm{L}$ isopropanol added on top of the gel to level the top edge of the gel. After $\sim 30 \mathrm{~min}$ isopropanol was removed and the $4 \%$ stacking gel cast on top of the resolving gel. A 10 -well comb was set in the stacking gel before polymerization. The stacking gel

Table 13 - Preperation of SDS gels (Volumes for $1 \mathrm{gel}$ with the Bio-Rad system)

\begin{tabular}{|l|c|c|c|c|}
\hline & $\begin{array}{c}4 \% \\
\text { stacking gel }\end{array}$ & $\begin{array}{c}10 \% \\
\text { resolving gel }\end{array}$ & $\begin{array}{c}12 \% \\
\text { resolving gel }\end{array}$ & $\begin{array}{c}15 \% \\
\text { resolving gel }\end{array}$ \\
\hline $\mathrm{dH} 20$ & $1.35 \mathrm{~mL}$ & $3.95 \mathrm{~mL}$ & $3.3 \mathrm{~mL}$ & $2.3 \mathrm{~mL}$ \\
\hline $30 \%$ Polyacrylamide & $335 \mu \mathrm{L}$ & $3.35 \mathrm{~mL}$ & $4 \mathrm{~mL}$ & $5 \mathrm{~mL}$ \\
\hline $1,0 \mathrm{M}$ Tris $(\mathrm{pH} 6.8)$ & $250 \mu \mathrm{L}$ & - & - & - \\
\hline $1,5 \mathrm{M}$ Tris $(\mathrm{pH} 8.8)$ & - & $2.5 \mathrm{~mL}$ & $2.5 \mathrm{~mL}$ & $2.5 \mathrm{~mL}$ \\
\hline $10 \%$ SDS & $20 \mu \mathrm{L}$ & $100 \mu \mathrm{L}$ & $100 \mu \mathrm{L}$ & $100 \mu \mathrm{L}$ \\
\hline $10 \%$ APS & $20 \mu \mathrm{L}$ & $100 \mu \mathrm{L}$ & $100 \mu \mathrm{L}$ & $100 \mu \mathrm{L}$ \\
\hline TEMED & $2 \mu \mathrm{L}$ & $4 \mu \mathrm{L}$ & $4 \mu \mathrm{L}$ & $4 \mu \mathrm{L}$ \\
\hline
\end{tabular}


accumulates the proteins from the wells so that they enter the stacking gel simultaneously. The brain homogenates diluted in Laemmli buffer were boiled for 5 min at $95^{\circ} \mathrm{C}$. After cool-down to RT, $50 \mu \mathrm{g}$ sample / well were loaded together with a pre-stained PAGE ruler. The homogenates were run in running buffer at $70 \mathrm{~V}$ until the samples had passed the stacking gel. After that the voltage was increased to $130 \mathrm{~V}$ and the gel run until the leading band of the samples had almost passed the resolving gel.

\section{Protein transfer}

After resolving, the proteins were transferred to a nitrocellulose or polyvinylidene difluoride (PVDF) membrane. In case of the PVDF membranes, membranes were briefly activated in methanol for $15 \mathrm{~s}$ and washed in distilled $\mathrm{H}_{2} \mathrm{O}$ for 3 min. Nitrocellulose and PVDF membranes were equilibrated in cold transfer buffer before the transfer. The polyacrylamid gel was removed from the gel glass plates and the transfer was set up in the Bio-Rad wet transfer system. A "sandwich" was formed in this order: transfer sponge, 1 layer of Whatman paper, gel, membrane, Whatman paper, transfer sponge. The sandwich was clamped together, removing all air bubbles in the process and put in a transfer chamber filled with transfer buffer and a cooling pad. Transfer was run at $100 \mathrm{~V}$ for $90 \mathrm{~min}$ at $4^{\circ} \mathrm{C}$.

Imaging of fluorescence-tagged membranes with the Odyssey Infrared Imager After the transfer, the membrane was removed from the sandwich and blocked in $5 \%$ milk for $1 \mathrm{~h}$ at RT. Several proteins of interest and a loading control were always stained on one membrane. For that reason, the membrane was cut, based on the protein ladder and the expected molecular weight of each protein. Primary antibodies were diluted according to manufacturer's specifications in $5 \%$ milk and incubated O/N at $4^{\circ} \mathrm{C}$ on a shaker. The next day, the membrane was briefly rinsed 3 times with trisbuffered salibe containing 1\% tween 20 (TBS-T) and then washed 3 times 10 min each with TBS-T. A fluorescence-tagged secondary antibody was used at a dilution of $1: 7500$ and incubated on the membrane for $1 \mathrm{~h}$ at RT in the dark while shaking. The membrane was then quickly rinsed 3 times with TBS-T and washed for 3 times 10 min with TBS-T. The membrane was briefly washed 3 times with TBS and then imaged on an Odyssey setup. The detected signal was exported as JPEG images 
and band density quantified using Fiji software. Membranes were dried between 2 Whatman papers and stored under dry conditions.

Chemiluminescence development of membranes

After transfer, the membranes were processed as for imaging on the Odyssee setup until the washing step after the primary antibody (s. above). An horseradish peroxidase (HRP)-conjugated secondary antibody was used at a dilution of 1:20001:5000 and incubated on the membrane for $50 \mathrm{~min}$ at RT while shaking. The membrane was then quickly rinsed 3 times with TBS-T and washed for 3 times 10 min with TBS-T. The membrane was briefly washed 3 times with TBS and then incubated with Luminata Western HRP substrate classic or forte for $3 \mathrm{~min}$. The signal was detected on X-ray films with an AGFA Curix 60 processor. The developed films were dried and scanned on an Epson Perfection V850 Pro Scanner. The band density was quantified with Fiji software. 


\section{Results}

\subsection{Characterization of motor behavior tests for aging mice}

This study was largely conducted in an endophilin-A (henceforth endophilin) deficient mouse model. There are three genes that encode for endophilin in mammals, therefore different genetic combinations of endophilin KOs were used to elucidate the influence of specific endophilin proteins on motor behavior and CNS morphology (Figure 20). The genotypes used for this thesis were endophilin 1KO-2WT-3WT mice, which have a normal lifespan and fertility and show no motor impairments, as well as endophilin 1KO-2HT-3WT, endophilin 1KO-2WT-3KO and endophilin 1KO-2HT-3KO mice, which have reduced lifespan and fertility and display seizures with aging (Figure 20).

To address how motor behavior changes with aging, the gait of BL6 WT animals was analyzed on a motorized treadmill. The setup was equipped with a high-speed video

\begin{tabular}{|c|c|c|c|c|}
\hline endophilin & $\begin{array}{l}\text { Endo } 1 K O-2 W T-3 W T \\
\text { Endo } 1 \mathrm{HT}-2 \mathrm{HT}-3 W \mathrm{~W} \\
\text { Endo } 1 \mathrm{HT}-2 \mathrm{HT}-3 \mathrm{HT}\end{array}$ & $\begin{array}{l}\text { Endo 1KO-2HT-3WT } \\
\text { Endo 1KO-2WT-3KO } \\
\text { Endo 1KO-2HT-2KO }\end{array}$ & $\begin{array}{l}\text { Endo 1KO-2KO-3WT } \\
\text { (DKO) }\end{array}$ & $\begin{array}{l}\text { Endo 1KO-2KO-3KO } \\
\text { (TKO) }\end{array}$ \\
\hline \multirow{2}{*}{$\begin{array}{r}\text { synaptojanin } \\
\text { dynamin }\end{array}$} & SJ WT & SJ HT & & SJ KO \\
\hline & dyn WT-KO & dyn HT-KO & & dyn KO-KO \\
\hline \multirow{3}{*}{$\begin{array}{r}\text { age limit } \\
\text { fertility } \\
\text { phenotype }\end{array}$} & $\begin{array}{l}\text { normal lifespan } \\
\text { normal reproduction }\end{array}$ & $\begin{array}{l}\text { shorter lifespan } \\
\text { (die by } 18 \text { months) } \\
\text { decreased fertility }\end{array}$ & die by P21 & die at PO \\
\hline & $\begin{array}{l}\text { no obvious } \\
\text { phenotype }\end{array}$ & $\begin{array}{l}\text { Mild to strong motor } \\
\text { impairments } \\
\text { (age-dependent) }\end{array}$ & $\begin{array}{l}\text { ataxia, } \\
\text { neurodegeneration } \\
\text { delayed development }\end{array}$ & $\begin{array}{l}\text { smaller than } \\
\text { littermates, } \\
\text { lack milk }\end{array}$ \\
\hline & $1 \mathrm{KO}-2 \mathrm{WT}-3 \mathrm{WT}$ & 1КО-2НT-2KO & $1 \mathrm{KO}-2 \mathrm{KO}-3 \mathrm{~V}$ & 1KO-2KO-3Ко \\
\hline
\end{tabular}

Figure 20 - The endophilin mouse model. Comparison of different endophilin-A mutants and the synaptojanin-1 and dynamin-1,3 mouse models. Complete KO of any of these endocytic proteins leads to death within a few hours after birth, while animals with partial deletion of the proteins have either a strong, mild or normal phenotype, depending on how many alleles are deleted in the model. Modified from (Murdoch, et al., 2016). 
camera under the transparent treadmill to record the underside of the animal while running (Figure $21 \mathrm{~A}$ ). From the recorded videos several gait parameters can be calculated. The principle of this calculation is to measure the paw area over time for every single paw (Figure $21 \mathrm{~B}$ ) and determine parameters that define gait rhythm and posture at an adjustable speed. Examples for these parameters are listed in Table 12.

A
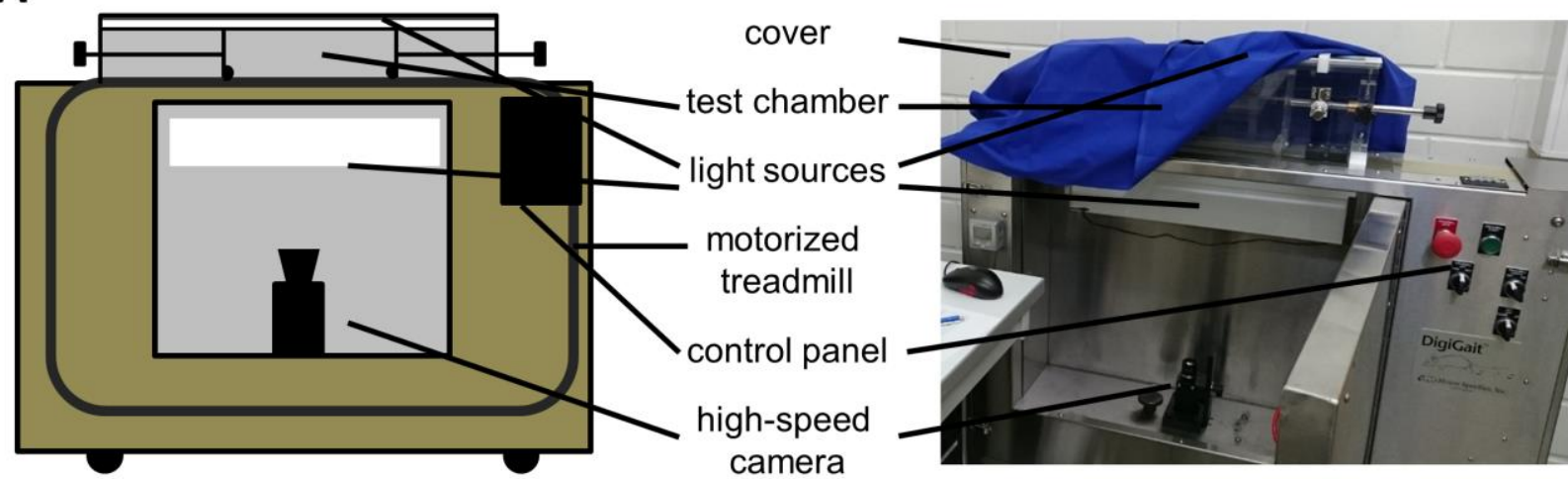

B

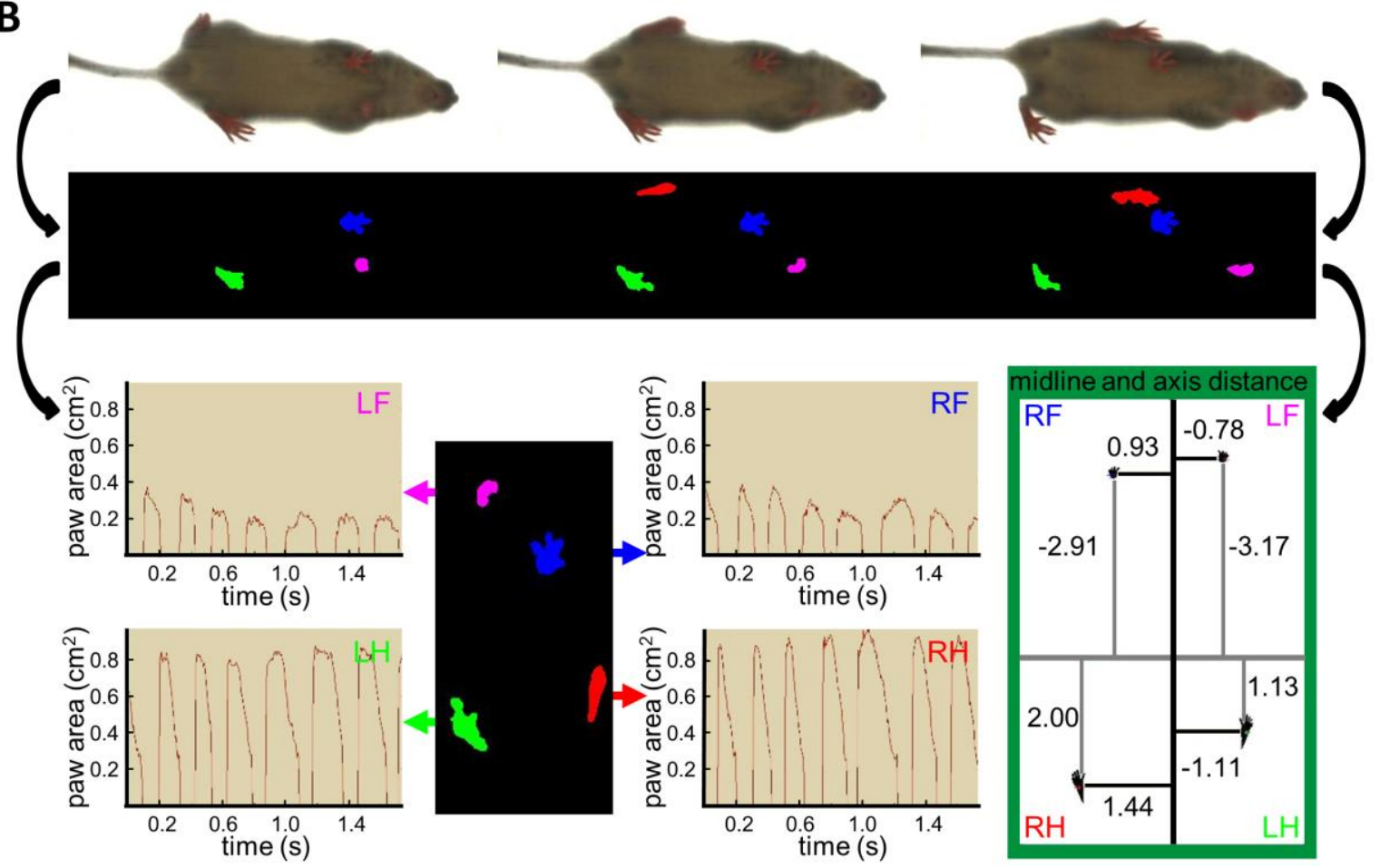

Figure 21 - Principle of gait recording. (A) Schematic drawing and photo of a DigiGait treadmill. (B) Principle of gait analysis on the DigiGait setup. The underside of the animal is filmed with a high-speed camera and the paw area over time determined. In addition paw positioning in relation to snout-tail axis and midline is determined. Modified from (Rostosky \& Milosevic, 2018). 
Analysis of young and aged WT animals showed that stance width did not change with age (Figure $22 \mathrm{~A}$ ) but stance/swing timing and limb loading did increased (Figure $22 \mathrm{~B}$ and D) and paw drag decreased with age (Figure $22 \mathrm{C}$ ). This shows that some aspects of gait are not affected by age while other parameters can be used to study normal and accelerated aging. As a result, gait analysis was added to a battery of motor behavior tests to describe aging and neurodegeneration in endophilin mutants.
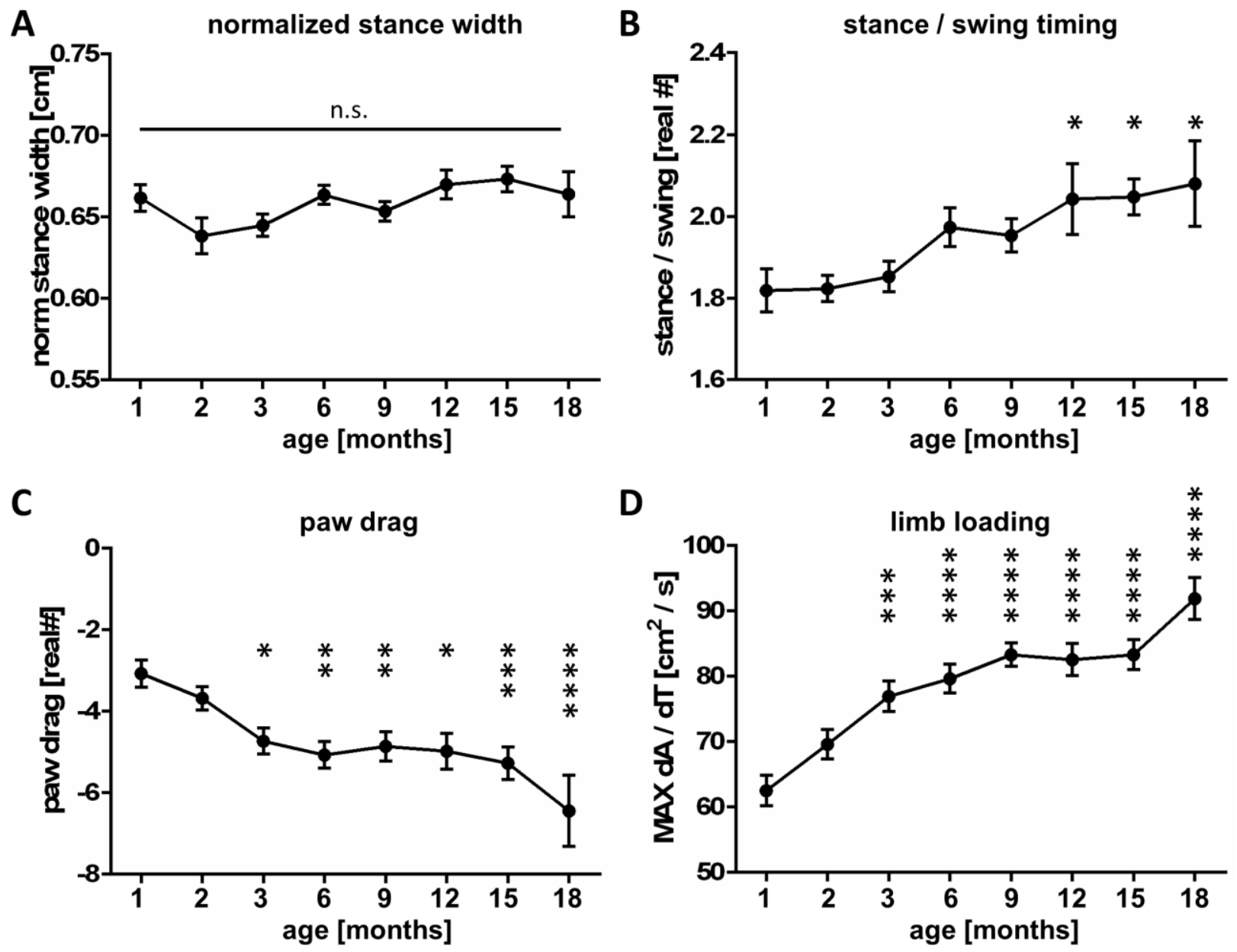

Figure 22 - Gait parameters change in aging WT mice. (A) Normalized stance width (distance between hind limbs / body width), (B) stance-swing timing (stance time / swing time), (C) paw drag (paw area between full stance and paw lift-off) and (D) limb loading (max dA/dt; maximal deceleration) of the hind limbs of BL6 WT animals from the age of $1-18$ months. Running speed $20 \mathrm{~cm} / \mathrm{s}$. Normalized stance width was stable over aging but stance/swing timing and limb loading increased over time while paw drag decreases with age. Significance by Dunnett-corrected one-way ANOVA (compared to 1 month) is displayed in the tables. ${ }^{* * * *}=p<0.0001$; ${ }^{* * *}=\mathrm{p}<0.001 ;{ }^{* *}=\mathrm{p}<0.01 ;{ }^{*}=\mathrm{p}<0.05$. 


\subsection{Motor behavior in endophilin-A mutants}

\subsubsection{Endophilin mutants display reduced muscle strength}

Motor behavior tests (gait analysis, Rotarod) were performed on endophilin mutants between the age of 1-18 months to minimize suffering of endophilin $1 \mathrm{KO}-2 \mathrm{HT}-3 \mathrm{KO}$ animals that display epileptic seizures and die between the ages of 18-20 months. Firstly, test animals were measured for body weight and muscle strength to assess general motor capacity to perform in motor behavior tests.

The body weight was found to increase throughout life in WT animals but was reduced in endophilin 1KO-2HT-3KO animals starting at an age of 9 months (Figure $23 \mathrm{~A}$ ). Other endophilin mutants had normal body weight compared to WT. The maximal grip strength was unchanged in endophilin mutants at a young age but reduced with aging starting already at an age of 3 months in endophilin 1KO-2HT-3KO mice (Figure 23 B). The grip strength normalized to body weight declined in WT animals over aging but declined faster in various endophilin mutants (Figure $23 \mathrm{C}$ ).
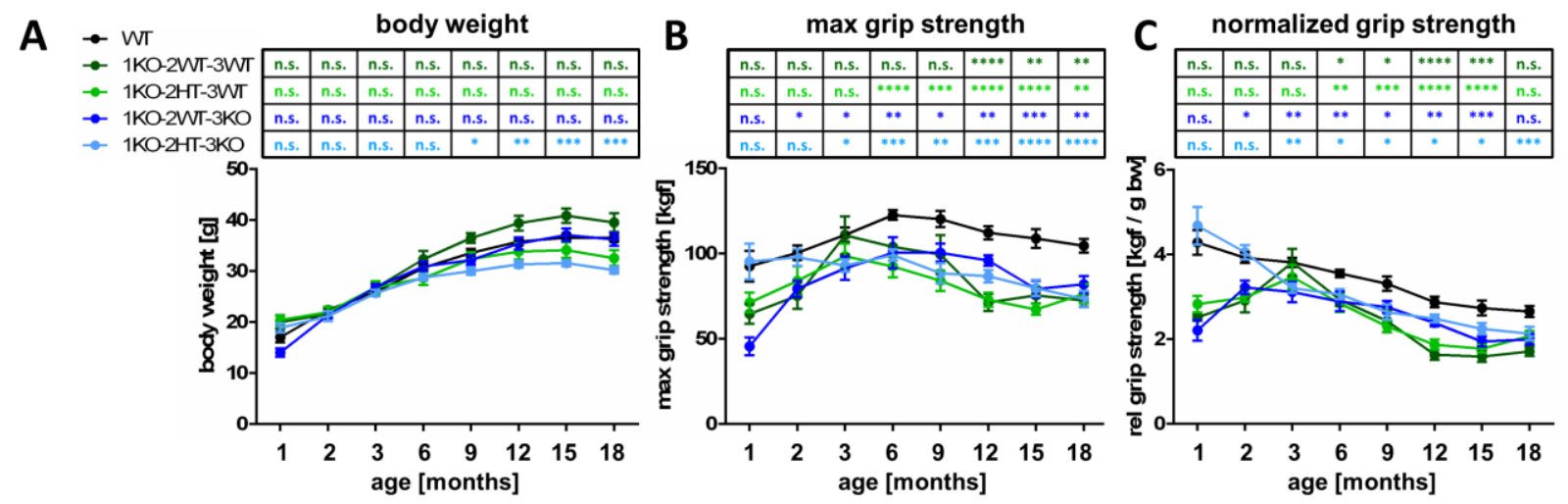

Figure 23 - Grip strength is reduced in endophilin mutants. (A) Body weight, (B) maximal grip strength and $(C)$ normalized grip strength (max grip strength / body weight) of WT (black), endophilin 1KO-2WT-3WT (dark green), 1KO-2HT-3WT (light green), 1KO-2WT-3KO (dark blue) and 1KO-2HT-3KO (light blue) mice over the age of 1-18 months. Graphs show mean \pm SEM. Significance by Dunnett-corrected oneway ANOVA (compared to WT) is displayed in the tables above the charts. ${ }^{* * * *}=p<0.0001 ;{ }^{* * *}=p<0.001 ;{ }^{* *}=p<0.01 ;{ }^{*}=p<0.05 . \mathrm{kgf}=$ kilogram force 


\subsubsection{Motor coordination is severely impaired in endophilin mutants}

To assess motor coordination and balancing, endophilin mutants were then subjected to Rotarod testing (Figure 24). In this test, animals were trained for two days for two trials of 180 seconds each during which the animals were running on a rod that rotated on a constant speed of 5 rotations per minute (rpm). During this fixed-speed Rotarod (FSRR) test, most endophilin mutants initially performed as well as WT animals but displayed decreased fall latency depending on the amount of missing endophilin alleles and their age. For example, endophilin 1KO-2WT-3WT mice performed as good as WT animals until the age of 18 months while $1 \mathrm{KO}-2 \mathrm{HT}-3 \mathrm{KO}$ animals performed significantly worse than WT animals already at an age of 1 months (Figure 24 A).

After the training phase, animals were tested on the accelerated Rotarod (ARR). Similar to the results of the FSRR, endophilin mutants showed decreased
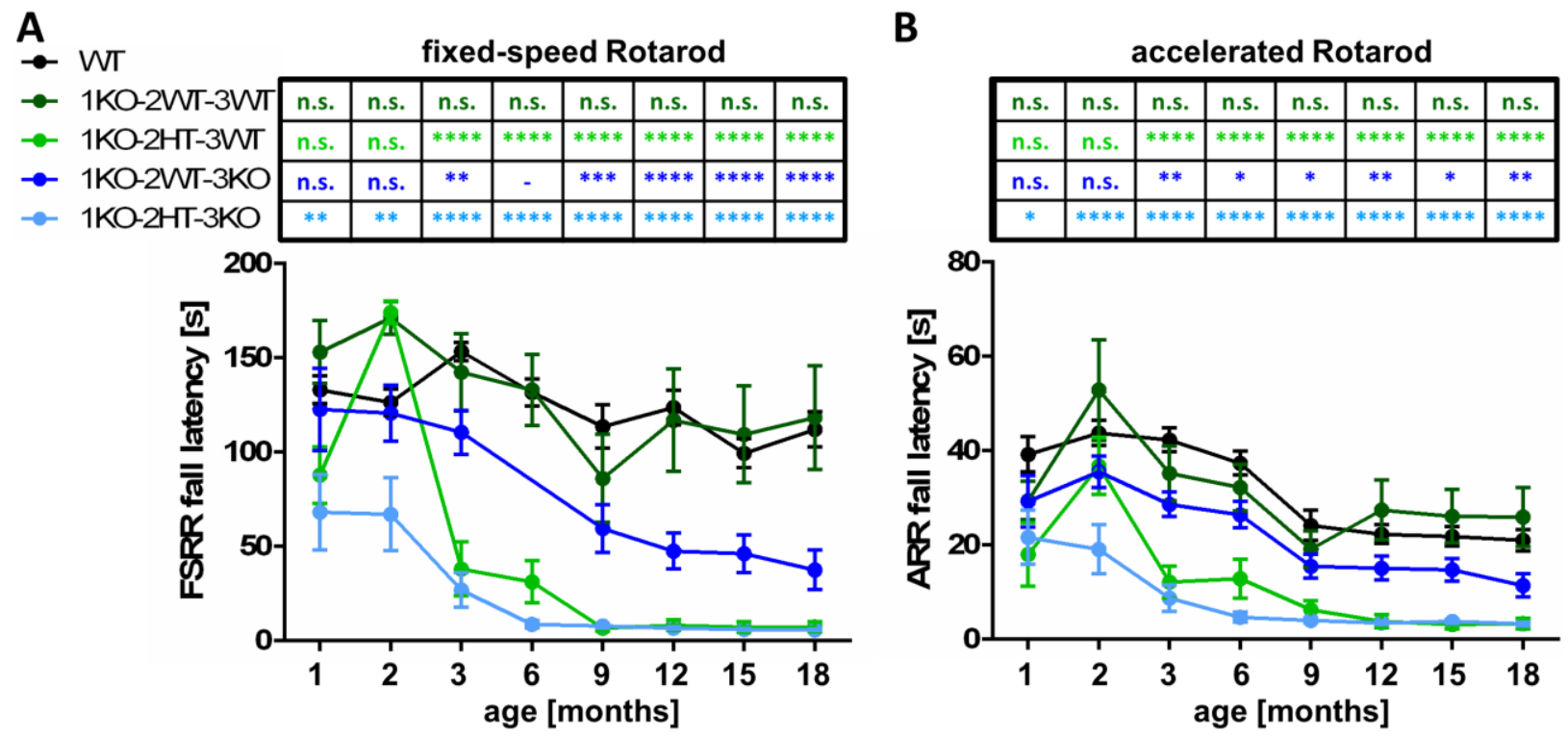

Figure 24 - Rotarod performance is impaired in endophilin mutants. Fall latency from the fixed-speed (A) and accelerated Rotarod (B). WT (black), endophilin 1KO2WT-3WT (dark green), 1KO-2HT-3WT (light green), 1KO-2WT-3KO (dark blue) and $1 \mathrm{KO}-2 \mathrm{HT}-3 \mathrm{KO}$ (light blue) mice were tested over the age of 1-18 months. Fall latency was significantly reduced in endophilin mutants depending on the number of missing endophilin alleles and age. Graphs show mean $\pm S E M$. The 6-month time point for endophilin 1KO-2WT-3KO mice was excluded from the analysis of FSRR due to technical problems at the test day. Significance by Dunnett-corrected one-way ANOVA (compared to WT) is displayed in the tables above the charts. ${ }^{* * *}=p<0.0001$; ${ }^{* * *}=\mathrm{p}<0.001 ;{ }^{* *}=\mathrm{p}<0.01 ;{ }^{*}=\mathrm{p}<0.05$. 
fall latency of the ARR (Figure $24 \mathrm{~B}$ ). Again, it can be observed that the more endophilin alleles are missing, the earlier the motor deficiencies are recorded in endophilin mutants.

Given endophilin's links to neurodegeneration and especially to PD (see chapter 1.7.1), endophilin mutants were tested by video based gait recording to detect gait abnormalities. On the DigiGait setup animals were recording running on a transparent
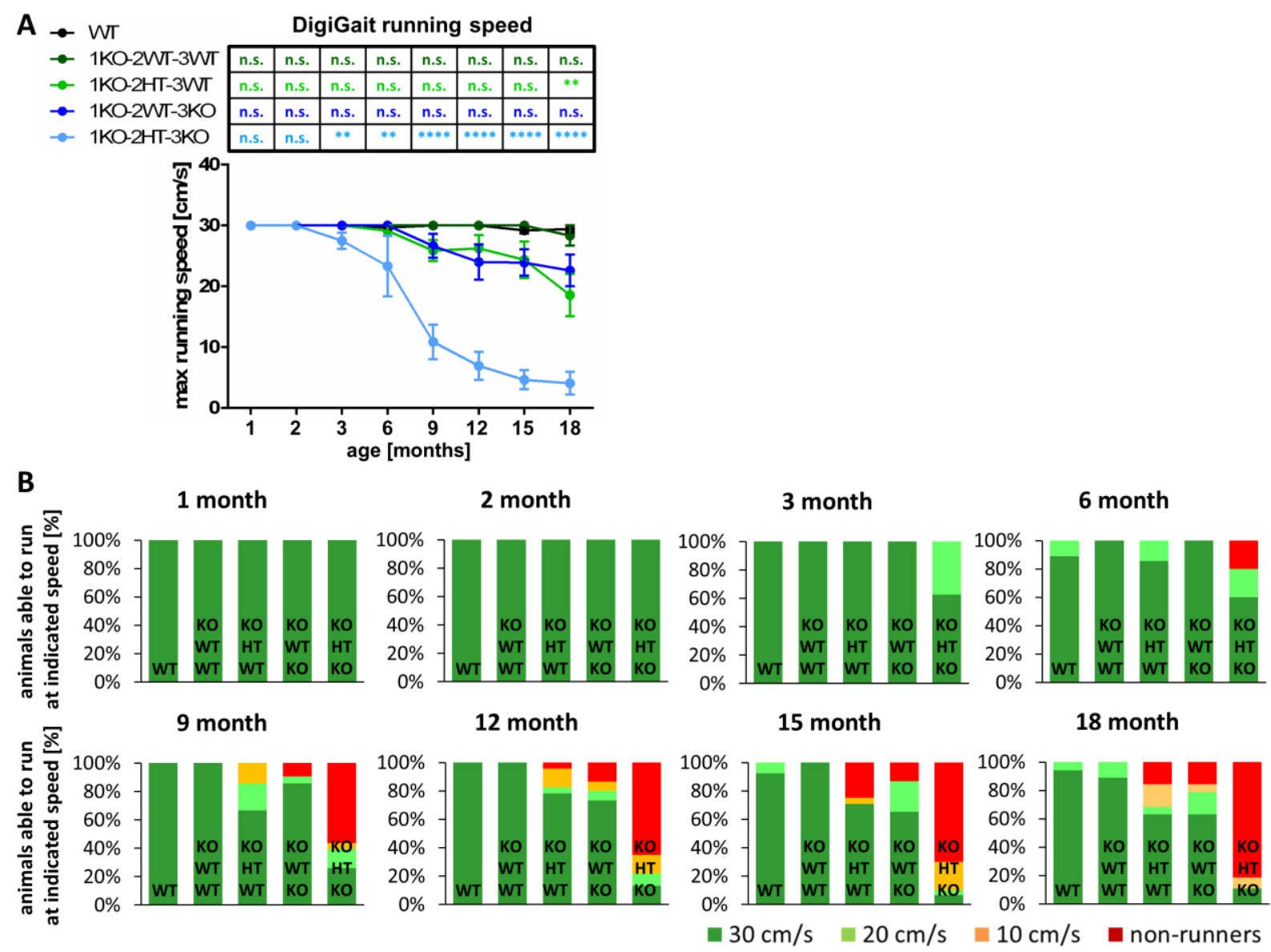

Figure 25 - Running speed is reduced in endophilin mutants. (A) Average running speed of WT (black), endophilin 1KO-2WT-3WT (dark green), 1KO-2HT-3WT (light green), 1KO-2WT-3KO (dark blue) and 1KO-2HT-3KO (light blue) animals on a DigiGait treadmill over the age of 1-18 months. Endophilin 1KO-2HT-3KO mice have a significantly reduced running speed from the age of 3 months. Graph shows mean $\pm S E M$. Significance by Dunnett-corrected one-way ANOVA (compared to WT) is displayed in the table above the chart. ${ }^{* * * *}=p<0.0001 ;{ }^{* * *}=p<0.001 ;{ }^{* *}=p<0.01$; ${ }^{*}=p<0.05$. (B) Percentage of animals able to run $30 \mathrm{~cm} / \mathrm{s}$ (dark green), $20 \mathrm{~cm} / \mathrm{s}$ (light green), $10 \mathrm{~cm} / \mathrm{s}$ (orange) or refuse to run on the DigiGait (red) for the indicated genotypes and ages. The percentage of animals refusing to run on the DigiGait increased continuously in endophilin 1KO-2HT-3KO mice starting from the age of 6 months. 
treadmill at 10, 20 and $30 \mathrm{~cm} / \mathrm{s}$. However, some endophilin mutants were not able to run at the maximum recording speed or refused running on the setup altogether (Figure 25).

Specifically, endophilin $1 \mathrm{KO}-2 \mathrm{HT}-3 \mathrm{KO}$ were capable of running at the highest tested speed at a young age, but $81.5 \%$ of these animals refused running at the age of 18 months (Figure $25 \mathrm{~B}$ ). Because of this, the running speed of $20 \mathrm{~cm} / \mathrm{s}$ was selected to perform a detailed gait analysis. This speed records the mice while running (unlike at $10 \mathrm{~cm} / \mathrm{s}$ which comes closer to walking than running) but includes more animals for analysis than videos recorded at $30 \mathrm{~cm} / \mathrm{s}$ as more animals are able to run at $20 \mathrm{~cm} / \mathrm{s}$.

From these videos recorded on the DigiGait setup, several parameters were calculated to describe gait in WT mice and the different endophilin mutants (as detailed in Rostosky \& Milosevic, 2018). A list of analyzed parameters is found in Table 12.

\subsubsection{Gait rhythm and posture are altered in endophilin mutants}

At a medium running speed of $20 \mathrm{~cm} / \mathrm{s}$, swing time (time the paw is in the air) and stance time (time the paw is in contact with the belt) were not changed in either fore limbs (Figure 26 A-B) or hind limbs (Supplemental Figure 1 A-B). However, during stance in fore limbs the propel time (time between max. paw area to paw lift-off) was reduced in all tested endophilin mutants in favor of brake time (time from paw first contact to the belt to max. paw area; Figure $26 \mathrm{C}-\mathrm{D}$ ) while these parameters were not changed in hind limbs (Supplemental Figure $1 \mathrm{C}-\mathrm{D}$ ). These data indicate that the gait rhythm is overall very similar in WT and endophilin deficient mice, though fine differences can be spotted in fore limb brake/propel timing.

Besides measuring basic gait rhythm, video based gait recording was used to describe the posture of the animal during running. One example for such a parameter is the paw angle (the angle of the paw to the snout-tail axis). The paw angle was decreased in all endophilin mutants while at the same time the paw angle variability (the standard deviation of the paw angle) was increased (Figure $27 \mathrm{~A}-\mathrm{B}$ ). Moreover, endophilin 1KO-2HT-3KO mice relied more often on shared stance (Figure $27 \mathrm{C}$ ) and 
had a decreased overlap of fore and hind paws on each side (Figure $27 \mathrm{D}$ ). This indicates that the posture of endophilin mutants is slightly changed during running.
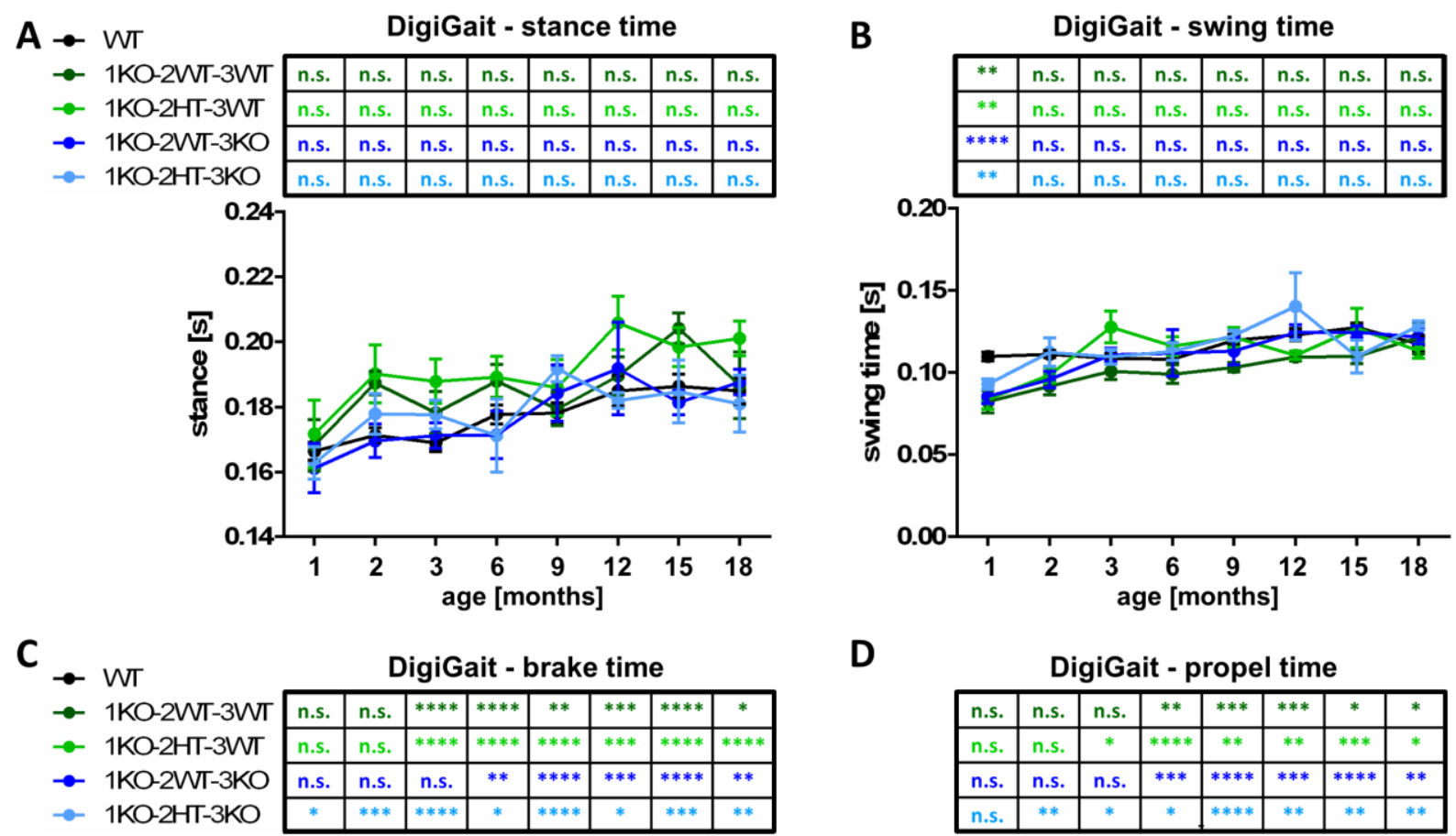

D

DigiGait - propel time
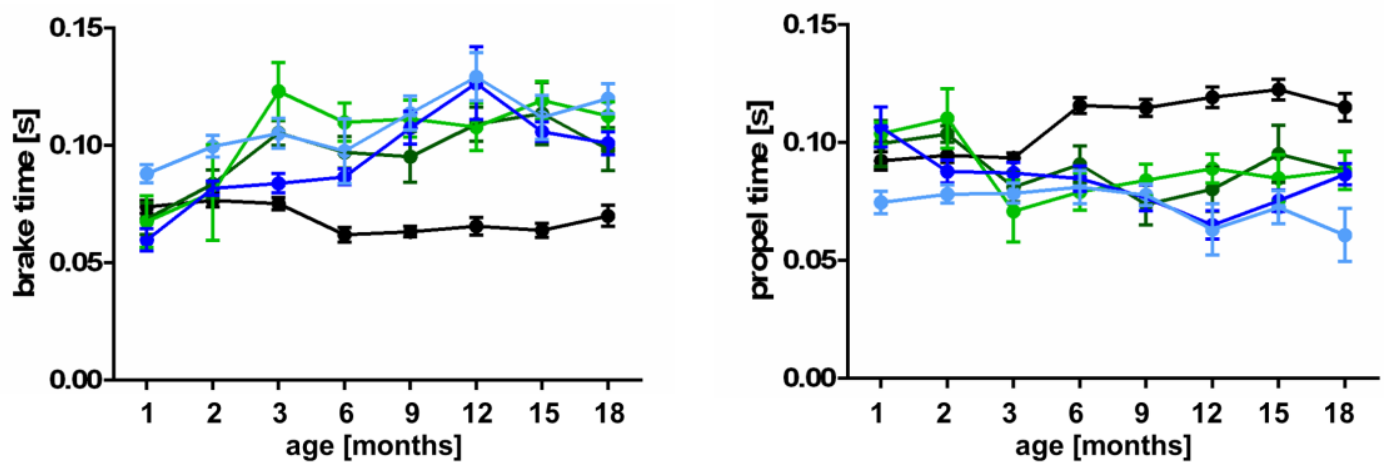

Figure 26 - Fore limb coordination is impaired in endophilin mutants. Gait timing parameters of WT (black), endophilin 1KO-2WT-3WT (dark green), 1KO-2HT-3WT (light green), 1KO-2WT-3KO (dark blue) and 1KO-2HT-3KO (light blue) animals recorded from the fore limbs on a DigiGait treadmill over the age of 1-18 months. (A) Stance time $=$ time the paw is in contact with the ground. (B) Swing time = time the paw is in the air. (C) Brake time = during stance, the time that the paw area is increasing. (D) Propel time = during stance, the time that the paw area is decreasing. While stance and swing time were not affected in endophilin mutants, brake time was increased in aged endophilin deficient animals and at the same time propel time was decreased. Running speed $=20 \mathrm{~cm} / \mathrm{s}$. Graphs show mean $\pm S E M$. Significance by Dunnett-corrected one-way ANOVA (compared to WT) is displayed in the tables above the charts. ${ }^{* * * *}=p<0.0001 ;{ }^{* * *}=p<0.001 ;{ }^{* *}=p<0.01 ;{ }^{*}=p<0.05$. 
In summary, endophilin deficient mutants displayed progressive motor impairments depending on the number of deleted endophilin alleles and the age. The impairments could be observed by a mild reduction of body weight and muscle strength and a very
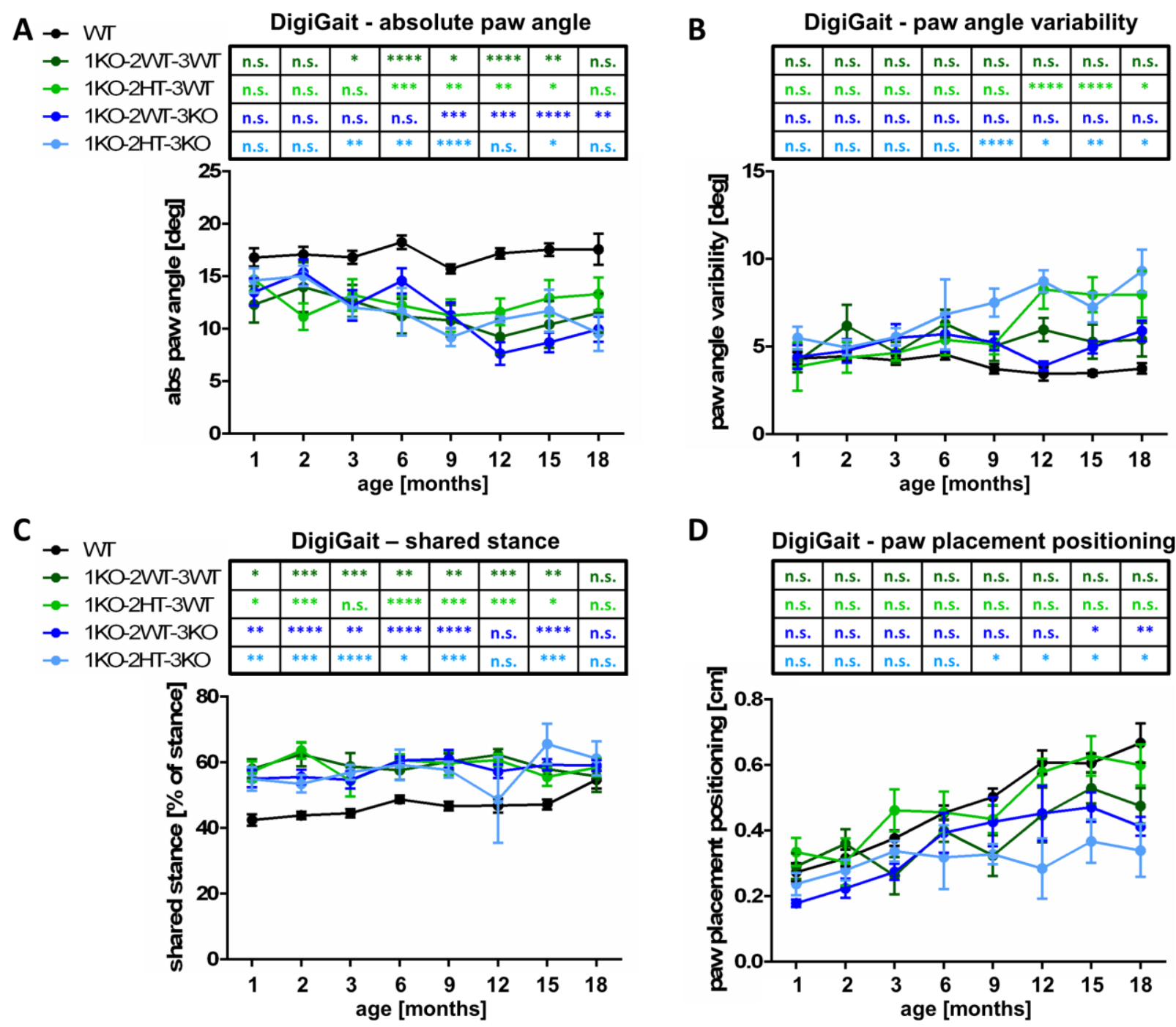

Figure 27 - Posture parameters are changed in running endophilin mutants. Hind limb posture parameters at $20 \mathrm{~cm} / \mathrm{s}$ for WT (black), endophilin 1KO-2WT-3WT (dark green), 1KO-2HT-3WT (light green), 1KO-2WT-3KO (dark blue) and 1KO-2HT3KO (light blue) animals recorded from the hind limbs on a DigiGait treadmill over the age of 1-18 months. (A) Paw angle = angle between the paw and the snout-tail axis. (B) Paw angle variability $=$ standard deviation of the paw angle. (C) Shared stance = time both hind limbs are in contact with the ground at the same time. (D) Paw placement positioning = extent of overlap between ipsilateral fore and hind paws during full stance. Running speed $=20 \mathrm{~cm} / \mathrm{s}$. Graphs show mean $\pm S E M$. Significance by Dunnett-corrected one-way ANOVA (compared to WT) is displayed in the tables above the charts. ${ }^{* * * *}=p<0.0001 ;{ }^{* * *}=p<0.001 ;{ }^{* *}=p<0.01 ;{ }^{*}=p<0.05$. 
strong reduction in general motor coordination (Rotarod) and running ability (running speed on the DigiGait). These changes were most obvious in endophilin $1 \mathrm{KO}-2 \mathrm{HT}$ $3 \mathrm{KO}$ mice which have only 1 out of 6 endophilin alleles left. In addition to the strong phenotype on the Rotarod, gait rhythm was mildly affected in the fore limbs of these animals and overall posture during running also deviated from WT controls. In summary, the motor deficits in endophilin mutants go beyond the normal motor deterioration in aging WT mice and point to a role of endophilin in controlling motor abilities.

\subsection{Motor behavior in other endocytic mutants}

To investigate if the observed motor behavior phenotype is shared by other mouse models for defective endocytosis, aged synaptojanin-1HT and dynamin $1 \mathrm{HT}-3 \mathrm{KO}$ mice were also tested by motor behavior tests. Similar to endophilin 1KO-2WT-3WT these animals have no apparent phenotype by home cage observation and have a normal life span.

\subsubsection{Synaptojanin-1 HT mice have no obvious motor impairments}

As synaptojanin-1 KO mice die within a few hours after birth, motor behavior tests were conducted on aged synaptojanin-1 HT animals and controls. Gait analysis showed that synaptojanin-1 HT and WT animals were able to reach the same maximum speed at 18 months of age (Figure $28 \mathrm{~A}$ ). Detailed analysis of gait parameters revealed no differences in key parameters such as the shared stance (Figure $28 \mathrm{~B}$ ). Further analysis revealed that neither muscle strength nor Rotarod performance were altered in synaptojanin-1 HT animals (Figure $28 \mathrm{C}-\mathrm{D}$ ). Overall, no motor impairments could be detected with the employed methods in aged synaptojanin-1 HT mice. 
A

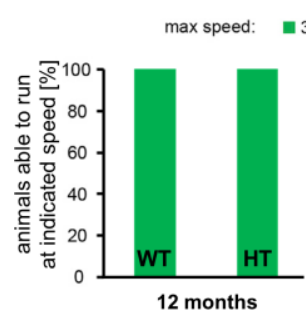

C

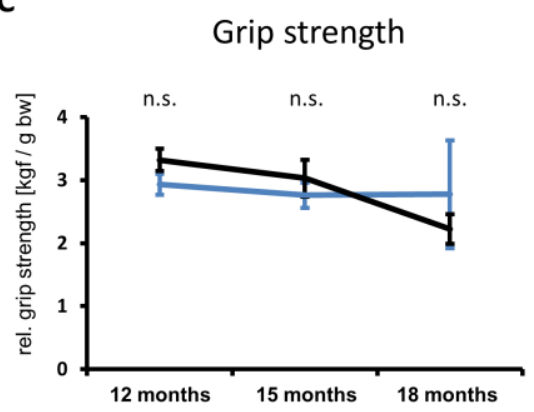

B shared stance

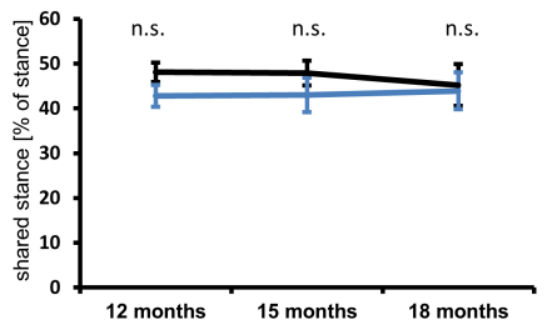

D

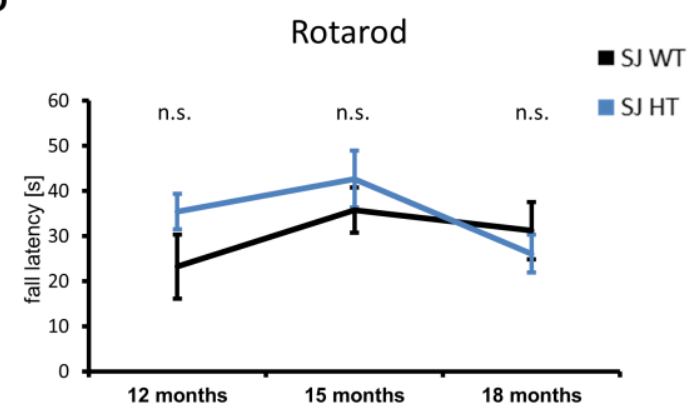

Figure 28 - Motor behavior is unaffected in synaptojanin-1 deficient mice. (A) Percentage of animals able to run $30 \mathrm{~cm} / \mathrm{s}$ (dark green) and $20 \mathrm{~cm} / \mathrm{s}$ (light green) for the indicated genotypes and ages. (B-C) Motor parameters for WT (black) and synaptojanin-1 HT (blue) animals at 12, 15 and 18 months of age. (B) Shared stance $=$ time both hind limbs are in contact with the ground at the same time at $20 \mathrm{~cm} / \mathrm{s}$. (C) Grip strength normalized to body weight. (D) Fall latency from the ARR. Graphs represent mean $\pm S E M$. No significant differences were detected between synaptojanin-1 WT and HT animals by two-tailed Student's t-tests.

\subsubsection{Dynamin mutants display mild motor impairments}

The other tested model used was dynamin-1,3 deficient animals. Like endophilin TKO and synaptojanin-1 KO animals, dynamin-1,3 DKO mice die shortly after birth and can hence not be used for motor behavior testing (Figure $20 \mathrm{~A}$ ). Motor behavior testing was thus performed on aged dynamin $1 \mathrm{HT}-3 \mathrm{KO}$ animals. These animals were able to run on the maximal running speed on the DigiGait treadmill both at young and older ages (Figure $29 \mathrm{~A}$ ). They displayed an increase in the percentage of shared stance as compared to WT animals at a young age but the difference was no longer observed at 18 months of age (Figure $29 \mathrm{~B}$ ) as the usage of shared stance also increased with aging in WT animals (compare also Figure $27 \mathrm{C}$ ).

On the other hand, grip strength was unaffected in the tested dynamin mutants at 3 months of age but was significantly lower in aged dynamin-1,3 mutants (Figure 29 C). 
Rotarod performance was significantly reduced at 3 months of age but remained on that level until the age of 15 months, while the performance of WT animals gradually decreased with aging (Figure 29 D, compare also Figure 24). At 18 months however, the ARR performance of dynamin-1,3 mutants was again significantly decreased as compared to WT (Figure 29 D).

These data show that aged synaptojanin-1 HT animals have no motor impairments that could be detected with the employed methods. Dynamin 1HT-3KO mice display mild motor impairments that become evident at a young age. However, while motor performance deteriorates with aging in WT animals, the motor abilities deteriorate much less of dynamin $1 \mathrm{HT}-3 \mathrm{KO}$ mice.

A

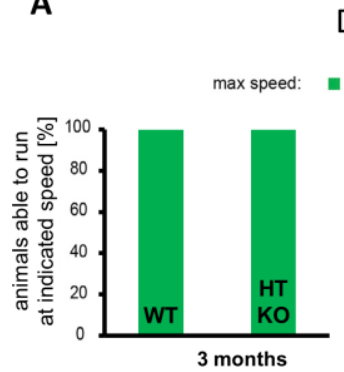

C

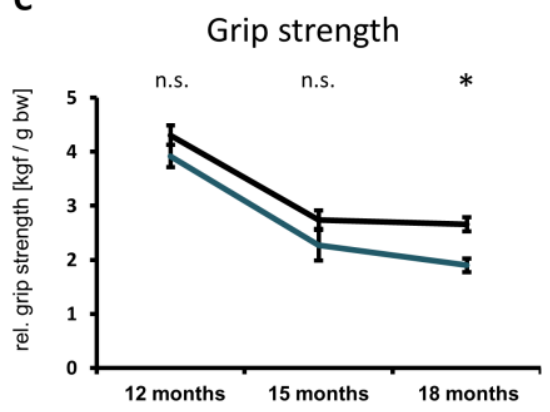

DigiGait running speed

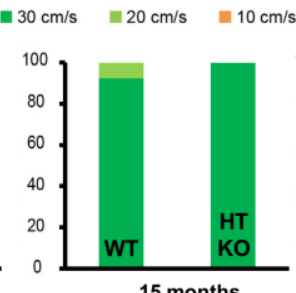

15 months

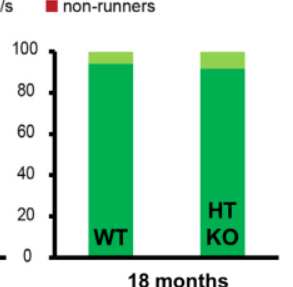

D

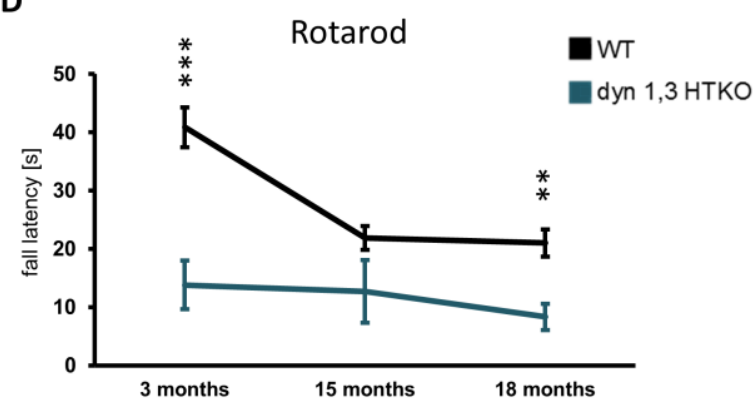

Figure 29 - Motor behavior is slightly impaired in dynamin-1,3 deficient mice. (A) Percentage of animals able to run $30 \mathrm{~cm} / \mathrm{s}$ (dark green) and $20 \mathrm{~cm} / \mathrm{s}$ (light green) for the indicated genotypes and ages. (B-C) Motor parameters for WT (black), and dynamin $1 \mathrm{HT}-3 \mathrm{KO}$ (turquois) animals at 3,15 and 18 months of age. (B) Shared stance $=$ time both hind limbs are in contact with the ground at the same time at 20 $\mathrm{cm} / \mathrm{s}$. (C) Grip strength normalized to body weight. (D) Fall latency from the ARR. Locomotion was not affected in aged dynamin mutants while grip strength and Rotarod performance was slightly decreased in dynamin mutant. Graphs represent mean $\pm S E M$. Significance stars represent ${ }^{* * *}=p<0.001 ;{ }^{* *}=p<0.01 ;{ }^{*}=p<0.05$ by twotailed Student's t-tests. 


\subsection{Cell death in endophilin mutants}

As defective endocytosis is strongly linked to various forms of neurodegeneration (see chapter 1.7.1) the different endophilin mutants were tested for cell death as a sign of neurodegeneration in the CNS. In the first step, endophilin 1,2 DKO (henceforth DKO), 18 month-old 1KO-2HT-3KO and appropriate control animals were perfused with PFA to pre-fixate the brain and avoid degradation. Cryosections of these brains were used for the stainings described in this section. Likewise, brains from $\mathrm{p} 0$ endophilin TKOs were dissected, frozen and used for stainings. Endophilin DKO and TKO animals have a significantly reduces lifespan (a few hours for TKOs and $\sim 21$ days for DKOs, see Figure 20) and are smaller than their littermates. DKO animals also display ataxia and neurodegeneration (Milosevic, et al., 2011).

\subsubsection{TUNEL staining reveals cell death in endophilin DKOs and aged 1KO- 2HT-3KO mice but not TKOs}

TdT-mediated dUTP-biotin nick end labeling (TUNEL) test was first performed on endophilin DKO brains to visualize all apoptotic and necrotic cells. Examination of different brain regions, including the cortex, hippocampus and cerebellum, revealed that while the number of TUNEL positive cells in WT and endophilin 1KO-2WT-3WT brains was extremely low, more TUNEL-positive cells were found in all brain regions of endophilin DKO mice (Figure 30).

TUNEL staining on endophilin TKO and control brains collected $~ 3-4$ hours after birth revealed a large number of apoptotic/necrotic cells both in control and endophilin TKO brains (Figure $31 \mathrm{~A}$ ). In fact, the number of TUNEL positive cells was slightly higher, yet not significantly so, in WT brains than in endophilin TKO brains. On the other hand, 18-month-old endophilin 1KO-2HT-3KO brains showed a low number of TUNEL-positive cells while no such cells were detected in WT cortex or hippocampi (Figure $31 \mathrm{~B}$ ). Together, these data show a slight increase in apoptotic and/or necrotic cells which cannot be observed in $p 0$ endophilin TKO brains, possibly due to high prevalence of apoptotic cells shortly after birth (Figure 31 A, WT signal). 

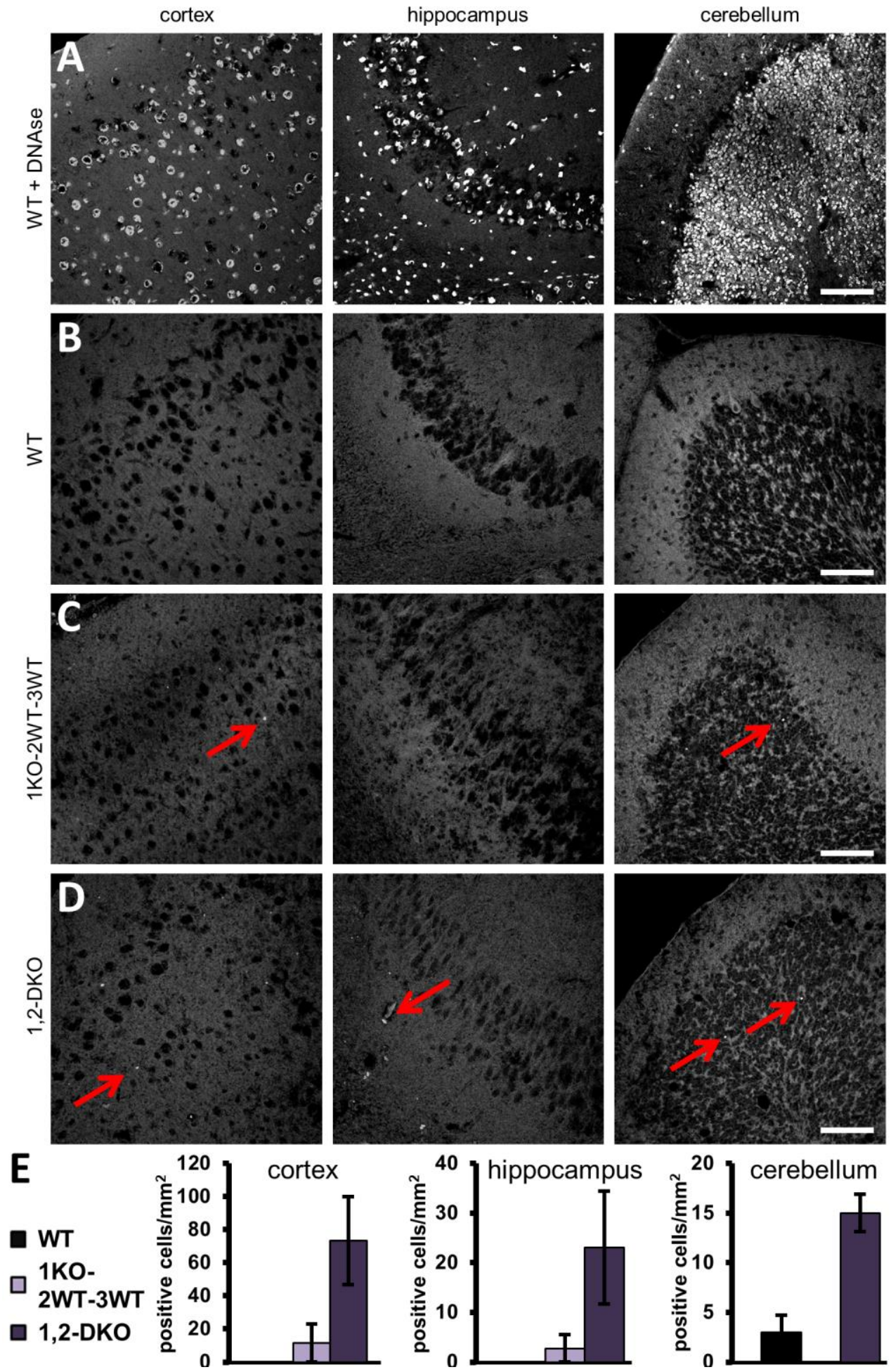

Figure 30 - Increase of apoptotic / necrotic cells in endophilin DKO brains. (AD) $10 \mu \mathrm{m}$ thick cryosections of p18 WT (B), endophilin 1KO-2WT-3WT (C) and endophilin DKO (D) brains stained by TUNEL. (A) WT brains treated by DNAse were used as positive control. Cortex (left), hippocampus (middle) and cerebellum (right) were imaged for TUNEL signal. Scale bars represent $50 \mu \mathrm{m}$. (E) Quantification of TUNEL signal in (B-D). Graphs represent mean \pm SEM. 
A
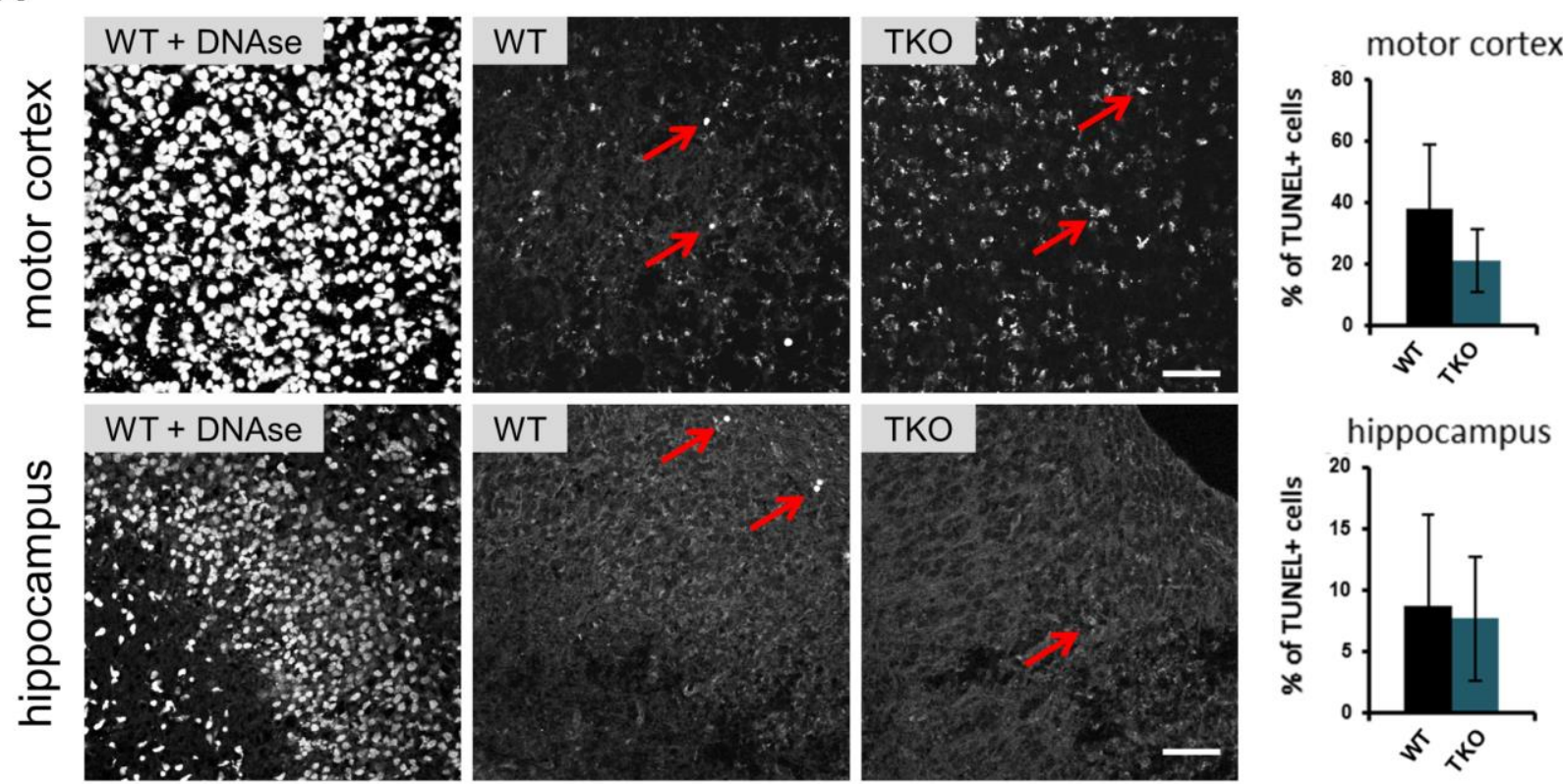

B
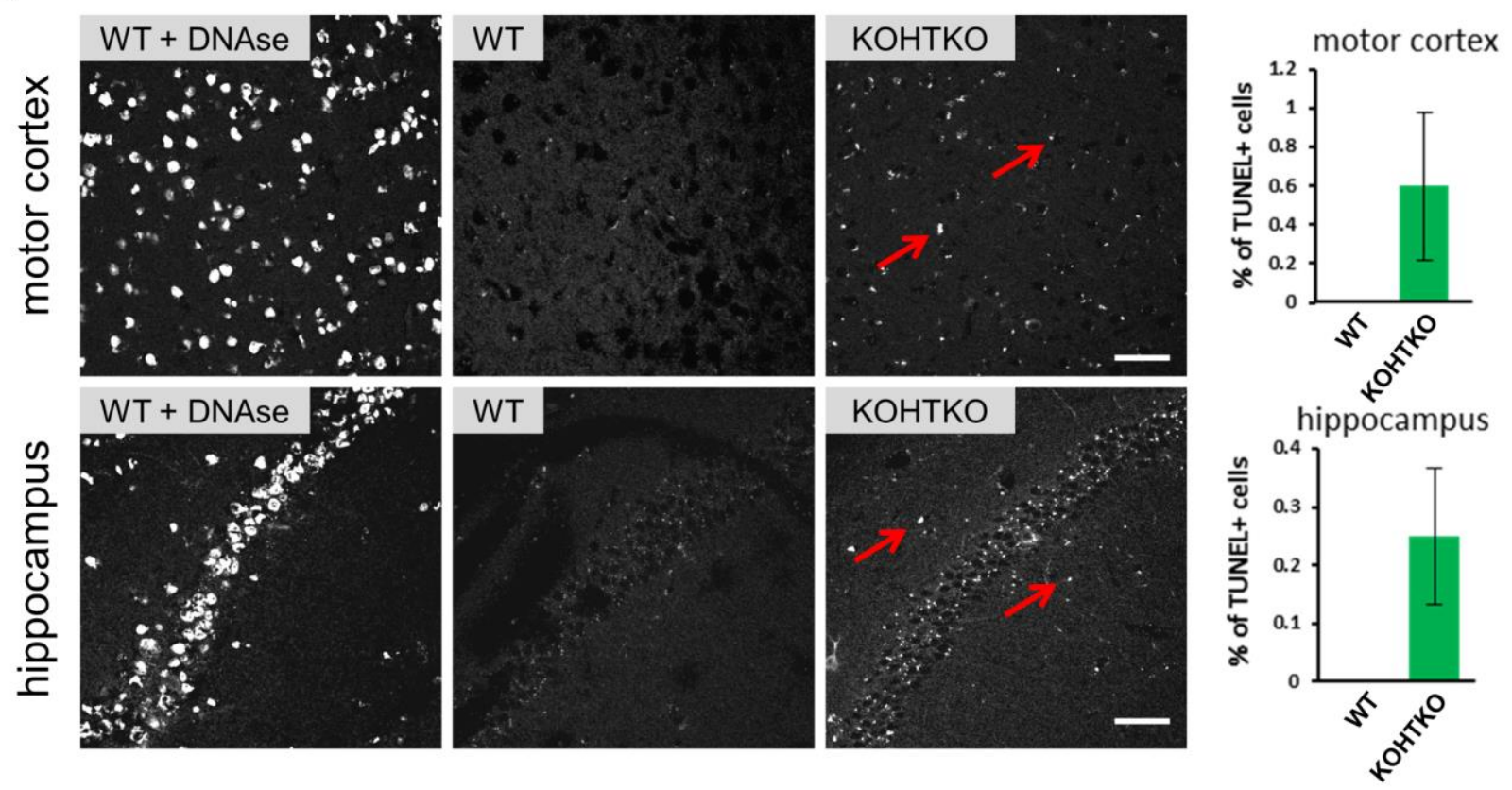

Figure 31 - Increase of apoptotic/necrotic cells in aged endophilin mutants. (A) $10 \mu \mathrm{m}$ thick cryosections of p0 WT and endophilin TKO brains stained by TUNEL. WT brains treated by DNAse were used as positive control (left). Motor cortex (top) and hippocampus (bottom) were imaged for TUNEL signal. (B) $10 \mu \mathrm{m}$ thick cryosections of 18-month-old WT and endophilin 1KO-2HT-3KO brains stained by TUNEL. WT brains treated by DNAse were used as positive control (left). Motor cortex (top) and hippocampus (bottom) were imaged for TUNEL signal. Scale bars represent $50 \mu \mathrm{m}$. (Right) Quantification of TUNEL signal. Graphs represent mean \pm SEM. 
As TUNEL staining revealed that there is an increase in necrotic/apoptotic cells in endophilin DKO and aged 1KO-2HT-3KO brains, cryosections from these animals were tested for glia cell activity. Glia cells aid in the removal of cell debris after cell death (reviewed in Khakh \& Sofroniew, 2015; Nayak, Roth, \& McGavern, 2014, see also chapter 1.1). Immunostaining for the astroglia cell marker glial fibrillary acidic protein (GFAP) showed a prominent increase of gliosis in p18 endophilin DKO brains (Figure $32 \mathrm{~A}$ ) that was not detected in 18-month-old endophilin $1 \mathrm{KO}-2 \mathrm{HT}-3 \mathrm{KO}$ brains (Figure $32 \mathrm{~B}$ ).

A

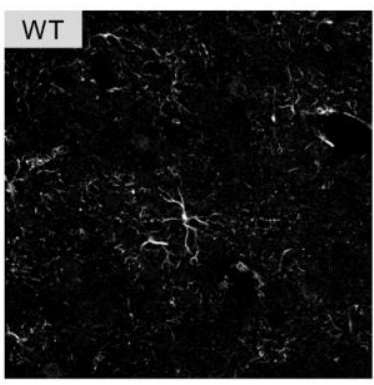

1KO-2WT
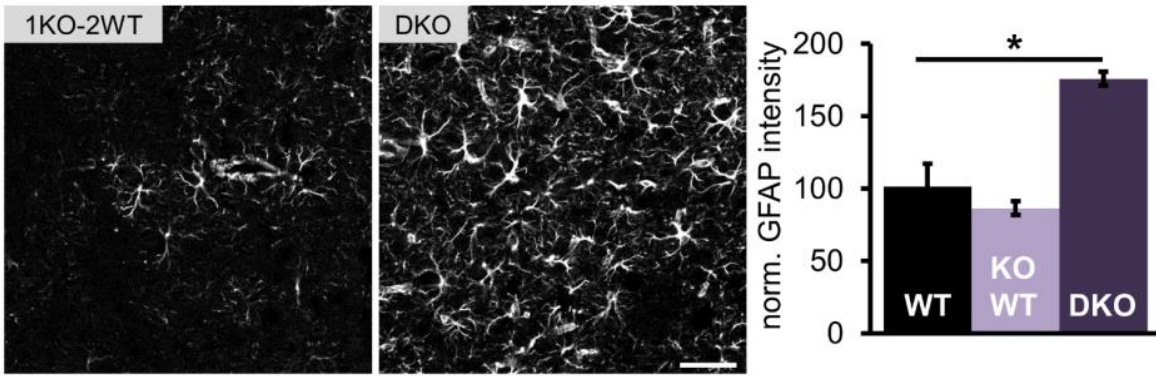

B
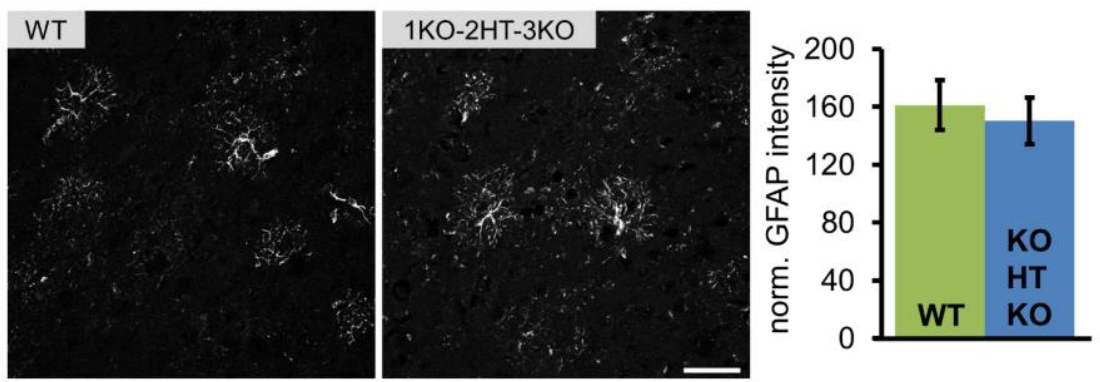

Figure 32 - Gliosis in the cortex of endophilin mutants. (A) $10 \mu \mathrm{m}$ thick cryosections of p18 WT, endophilin 1KO-2WT-3WT and endophilin DKO brains immunostained for the glial fibrillary acidic protein (GFAP). (B) $10 \mu \mathrm{m}$ thick cryosections of 18-month-old WT and endophilin 1KO-2HT-3KO brains immunostained for GFAP. Scale bars represent $50 \mu \mathrm{m}$. (right) Quantification of GFAP intensity. GFAP intensity was increased in endophilin DKO but not in endophilin 1KO$2 \mathrm{HT}-3 \mathrm{KO}$ brain sections. Graphs represent mean \pm SEM. ${ }^{*}=\mathrm{p}<0.05$ by two-sided Student's t-test. 


\subsubsection{The apoptosis marker caspase 3 is enriched in endophilin but not in synaptojanin-1 brain sections}

Given the high level of TUNEL signal in WT brain sections, an increase in cell death in endophilin TKO brains might be undetectable by the TUNEL assey because apoptosis occurs as part of normal development at p0 also in WT brains and may obscure any abnormal increase in apoptosis. To address this possibility, brains were immunostained for caspase 3 activity. Cleavage of caspase- 3 is an early step in apoptosis (Porter \& Jänicke, 1999). Immunostainings on cryosections showed that the number of cleaved caspase 3 postive cells was significantly increased in endophilin TKO brains (Figure 33). This finding suggests that there is an increase in apoptotic activity in endophilin TKO brains.

To understand whether the increase in caspase 3 activity is a commen feature of endocytic deficient animals, brain sections from synaptojanin-1 $\mathrm{KO}$ mice were also subjected to caspase 3 immunostainings. These stainings show that while cleaved caspase 3 signal does trend up in synaptojanin-1 KO brains, the difference was not significant (Figure 34).
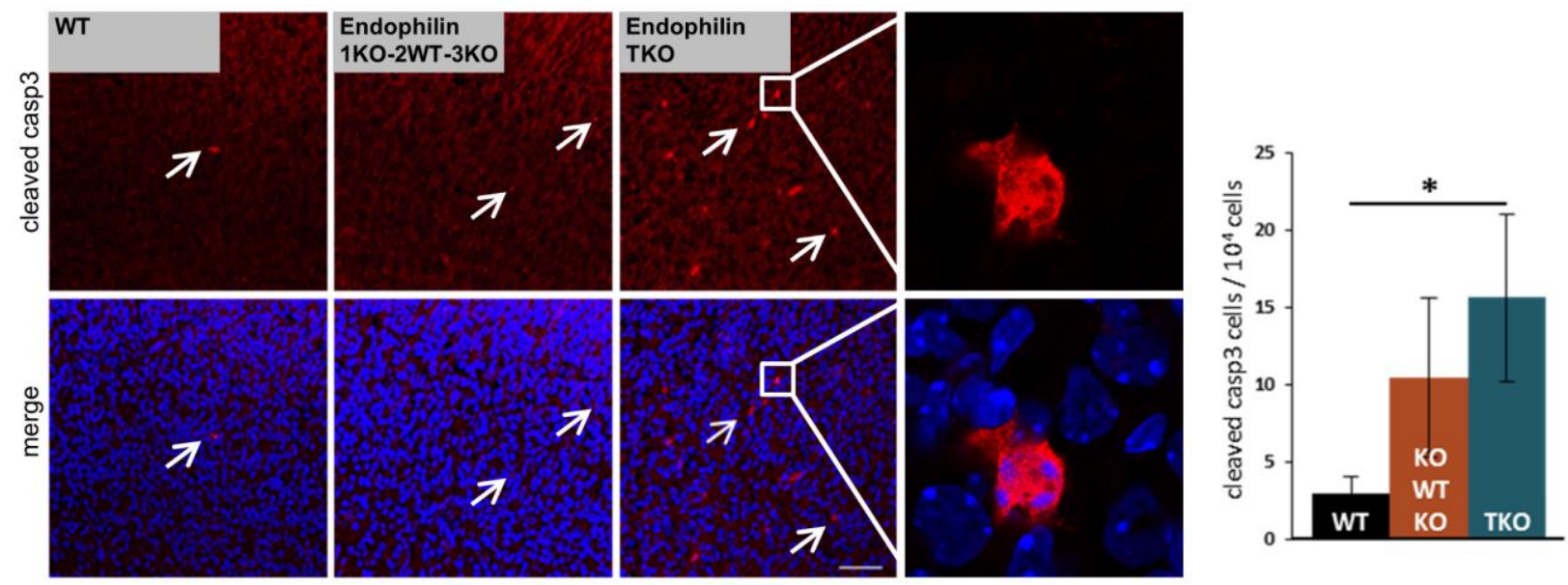

Figure 33 - Increased apoptotic activity in the endophilin TKO cortex. $10 \mu \mathrm{m}$ thick cryosections of p0 WT, endophilin 1KO-2WT-3KO and endophilin TKO brains immunostained for cleaved caspase 3. DAPI co-staining. Scale bar represents $50 \mu \mathrm{m}$. Insert shows one cleaved caspase 3 positive nucleus. (Right) Quantification of TUNEL signal. Graphs represent mean \pm SEM. Significantly more cleaved caspase 3 positive cells were found in endophilin TKO brains compared to WT. ${ }^{*}=p<0.05$ by onesided Student's t-test. 


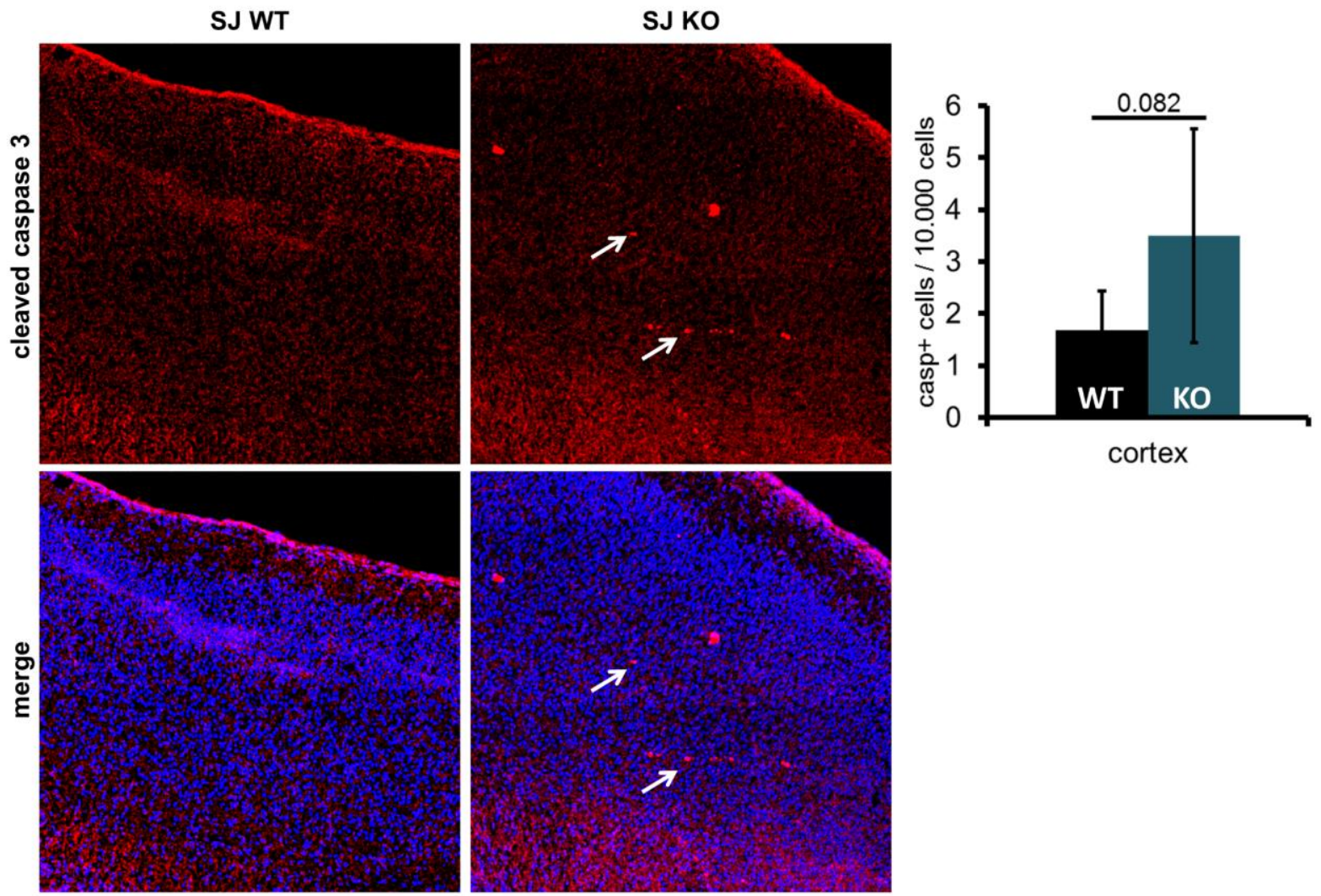

Figure 34 - No increase of apoptotic activity in synaptojanin-1 deficient cortex. $10 \mu \mathrm{m}$ thick cryosections of p0 WT and synaptojanin-1 KO brains immunostained for cleaved caspase 3. DAPI co-staining. Scale bar represents $50 \mu \mathrm{m}$. (Right) Quantification of TUNEL signal. Graphs represent mean \pm SEM. The number of cleaved caspase 3 positive cells in synaptojanin-1 KO sections trended compared to WT. No significant differences were found by one-sided Student's t-test.

\subsubsection{A-synuclein levels and distribution are altered in endophilin DKOs}

Given the links of defective endocytosis to PD (see chapter 1.7.1) and the observed motor deficits in endophilin deficient mice (see chapter 3.2), a prominent factor for $\mathrm{PD}, \alpha$-synuclein, was investigated in endophilin DKO brains. A-synuclein is found in protein aggregates throughout the brain of most PD patients. To test whether $\alpha-$ synuclein forms aggregates or is enriched in the endophilin mouse model, brain sections were stained for $\alpha$-synuclein. Overview stainings of the hippocampus showed a decrease in overall $\alpha$-synuclein intensity compared to littermate controls (Figure $35 \mathrm{~A}$ ). Interestingly, further investigation of $\alpha$-synuclein puncta showed no 


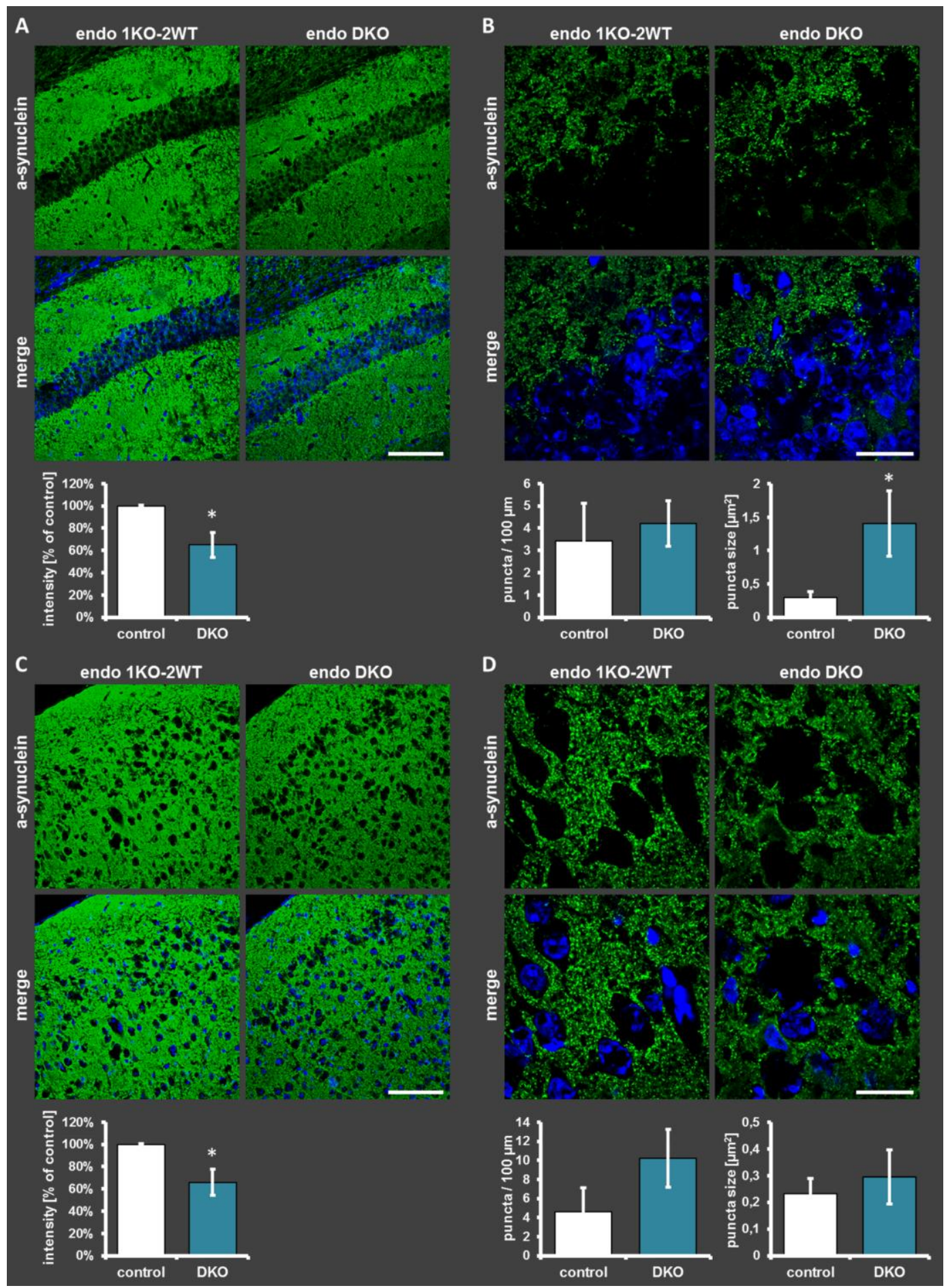

Figure 35 - A-synuclein decreases in endophilin DKO brains and forms bigger puncta. Immunostainings for $\alpha$-synuclein of $10 \mu \mathrm{m}$ thick cryosection from p18 endophilin 1KO-2WT-3WT and endophilin DKO brains. Overview $(A, C)$ and detailed recording $(B, D)$ of the hippocampus $(A-B)$ and motor cortex (C-D) $(A$ and $C)$ Scale bar $400 \mu \mathrm{m}$. A-synuclein intensity is quantified in the graphs. (B and D) Scale bar 40 $\mu \mathrm{m}$. Number of $\alpha$-synuclein puncta and puncta size is quantified in the graphs. Graphs represent mean \pm SEM. ${ }^{*}=p<0.05$ by two-sided Student's t-test. 
increase in the number of $\alpha$-synuclein puncta (Figure $35 \mathrm{~B}$ ). However, the average size of these puncta was significantly higher in endophilin DKO than in control hippocampi. The decrease in a-synuclein intensity was also found in the cortex of endophilin DKOs, but there was no change in a-synuclein puncta in this brain region (Figure $35 \mathrm{C}-\mathrm{D}$ ).

To look into more subtle changes of brain morphology, the Golgi silver staining method, which randomly stains single cells in the nervous system, was used on endophilin DKO mice. Brain sections were imaged and processed with the Imaris filament tracer (Figure $36 \mathrm{~A}$ ). The spine density as well as the spine classification was analyzed. However, no significant changes in the spine density or the number of mature mushroom-type spines were found in the hippocampus and substantia nigra of endophilin DKO brains (Figure $36 \mathrm{~B}$ ).
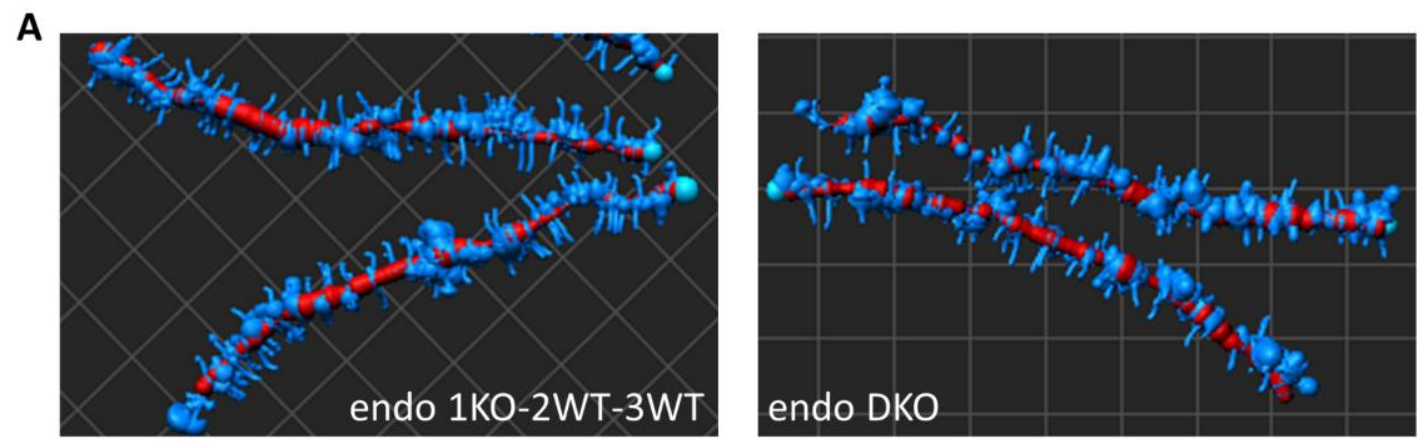

B
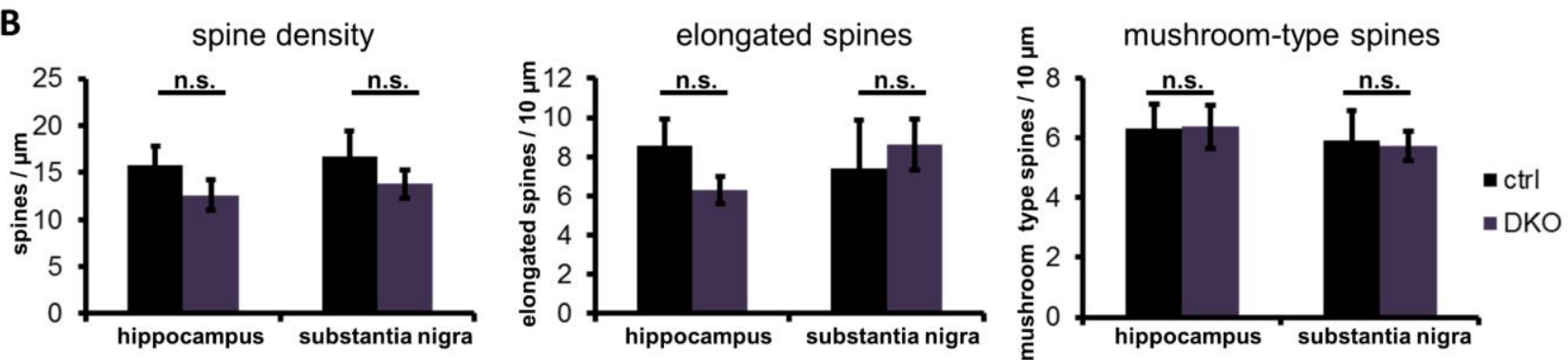

Figure 36 - Spine density and size are not changed in endophilin DKO mutants. (A) Reconstruction of dendritic spines in the substantia nigra by Imaris filament tracer of p18 endophilin 1KO-2WT-3KO and DKO brain sections. (B) Spine analysis of endophilin DKO and littermate controls. Spine density = number of spines $/ \mu \mathrm{m}$ dendrite. Elongated spines $=$ spines that have a mean head width $\geq$ mean neck width and length $>2 \mu \mathrm{m}$. Mushroom type spines = spines that have a max head width $>$ mean neck width $\times 1.5$. Graphs represent mean \pm SEM. No significant changes were found in endophilin DKO brain sections by two-sided Student's t-test. 
In summary, endophilin mutants show an increase in cell death by TUNEL staining in endophilin DKO and 18-month-old endophilin 1KO-2HT-3KO brains that in case of the endophilin DKOs is accompanied by gliosis. In p0 endophilin TKO brains, an increase of caspase 3 activity could be detected, that was not observed in synaptojanin-1 KO brains. In addition, immunostainings for the PD associated protein $\alpha$-synuclein showed differences in the levels and distribution in endophilin DKO brain sections. However, dendritic spine analysis revealed no difference in these animals. 


\subsection{Autophagy impairments in endophilin mutants}

NGS analysis of endophilin TKO and DKO brains has revealed that the most strongly up-regulated gene in endophilin deficient brains is the E3 ubiquitin ligase Fbxo32 (Murdoch, et al., 2016; see also chapter 1.4.1). Fbxo32 up-regulation was further confirmed by quantitative PCR and western blot analysis of endophilin TKO and DKO brains (Murdoch, et al., 2016).

\subsubsection{Fbxo32 is misregulated in aged endophilin 1KO-2HT-3KO mice}

Brain homogenates from aged endophilin 1KO-2HT-3KO mice were thus analyzed by western blot and it was found that Fbxo32 is not changed at the age of 3 or 9 months, but in fact become up-regulated by the age of 18 months (Figure 37 ).

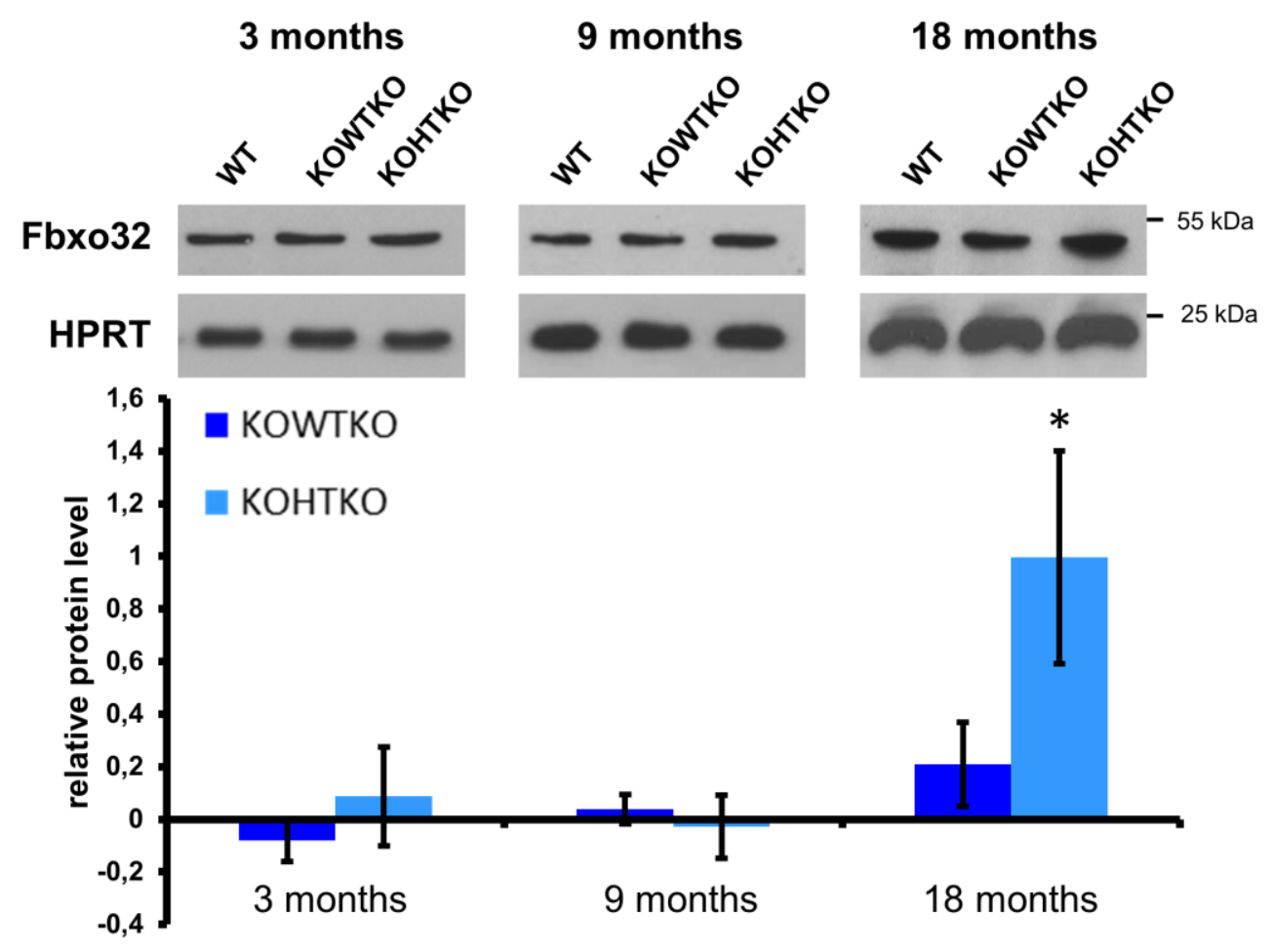

Figure 37 - Fbxo32 protein levels are increased in aged endophilin 1KO-2HT$3 \mathrm{KO}$ brains. Fbx032 levels relative to HPRT loading control was assessed by western blot at 3, 9 and 18 months. Change toward WT is shown in the graph. Fbxo32 is upregulated in 18-month-old endophilin 1KO-2HT-3KO mice. Graphs represent mean fold change vs WT \pm SEM. ${ }^{*}=p<0.05$ by two-sided Student's t-test. 
The expression of Fbxo32 is regulated by the transcription factors Foxo1 and Foxo3A (Sandri, et al., 2004). Foxo3A was also found to be up-regulated in endophilin TKO and DKO brains by NGS analysis, while Foxo1 levels are unchanged (Murdoch, et al., 2016). Of interest, the phosphorylation of Foxo3A at Ser253, which inhibits its transcriptional activity, was found to be decreased (Murdoch, et al., 2016). Immunostaining for this inhibitory phosphorylated form of Foxo3A in endophilin TKO cryosections also showed an altered distribution of Foxo3A in the hippocampus where it was accumulated in small, high-intensity puncta (Figure 38). These findings suggest that the transcriptional regulation of Fbxo32 is functioning abnormally and thereby favor high expression levels of Fbxo32 in endophilin mutants.

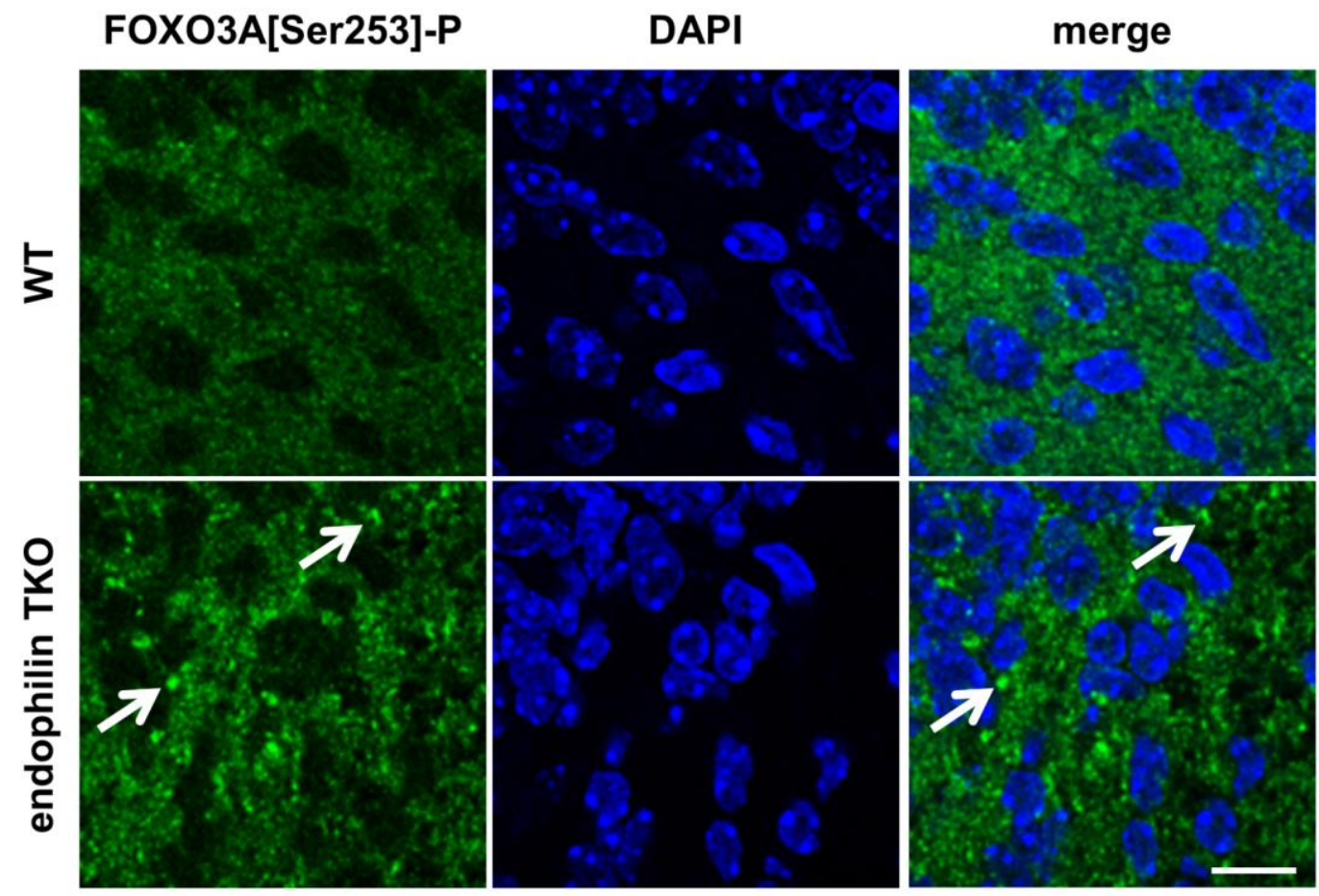

Figure 38 - Foxo3A-P mislocalizes in endophilin TKO brains. Immunostaining for Foxo3A(Ser253)-P (green), co-stained for DAPI (blue) in the hippocampus of p0 WT (top) and endophilin TKO (bottom) cryosections. Phosphorylated Foxo3A formed puncta that give a high intensity signal in endophilin TKO brain sections. Scale bar represents $10 \mu \mathrm{m}$. 


\subsubsection{Poly-ubiquitinated proteins accumulate in endophilin mutants but not in} synaptojanin-1 or dynamin deficient brains

As Fbxo32 is an E3 ubiquitin ligase that targets proteins for degradation through the UPS (see chapter 1.6.2), brains from endophilin DKO and TKO mice were analyzed for levels of poly-ubiquitinated proteins. To this end, brain homogenates were analyzed by western blots using three distinct markers of poly-ubiquitination: total poly-ubiquitin, K48-linkage specific poly-ubiquitin and K63-linkage specific polyubiquitin. K48- and K63- specific antibodies reflect different systems of the lysine

A
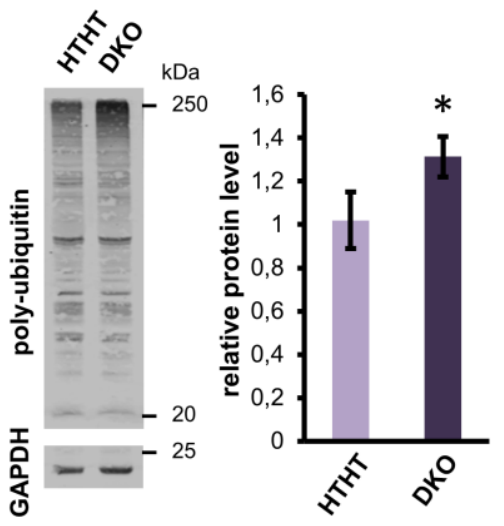

B

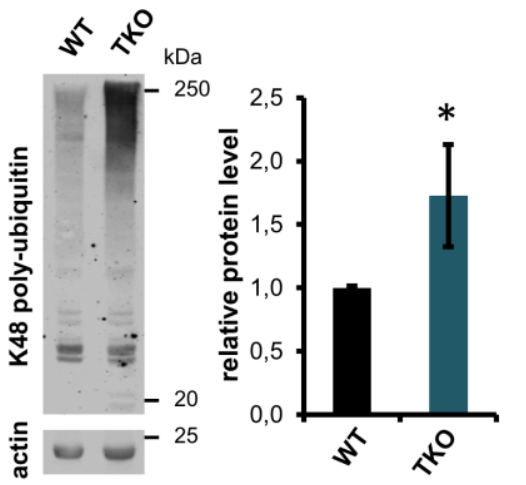

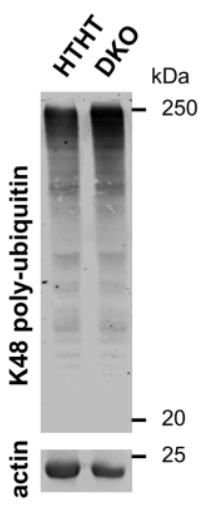
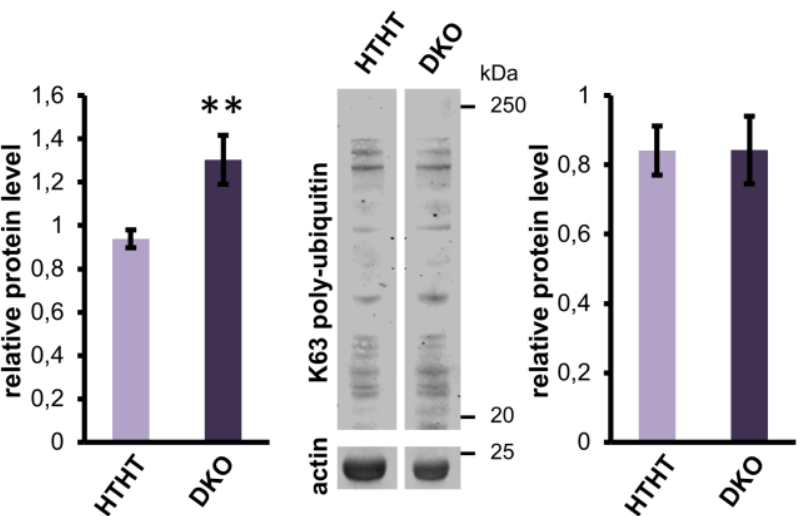

Figure 39 - Increase of poly-ubiquitinated proteins in endophilin DKO and TKO brains. (A) Relative protein levels (normalized to GAPDH or actin) of total polyubiquitin (left), K48 linkage specific poly-ubiquitin (middle) and K63 linkage specific poly-ubiquitin (right) in p18 endophilin 1HT-2HT-3WT and endophilin DKO brain homogenates was assessed by western blot analysis. (B) Relative protein levels (normalized to actin) of K48 linkage specific poly-ubiquitin (left) and K63 linkage specific poly-ubiquitin (right) in p0 WT and endophilin TKO brain homogenates was assessed by western blot analysis. Total poly-ubiquitination as well as K48 specific poly-ubiquitination was increased in the endocytic mutants, while K63 specific polyubiquitination was unchanged. Graphs represent mean \pm SEM. ${ }^{* *}=p<0.01 ;{ }^{*}=p<0.05$ by two-sided Student's t-test. 
based elongation of the ubiquitin tail. While K48-linkage of ubiquitin marks proteins for degradation through the UPS, K63-linkage plays a role in signaling (Sadowski, Suryadinata, Tan, Roesley, \& Sarcevic, 2012; Pickart \& Fushman, 2004; BenSaadon, Zaaroor, Ziv, \& Ciechanover, 2006; Silva, Finley, \& Vogel, 2015; see also chapter 1.5.5).

In endophilin DKO brains, total poly-ubiquitin levels were significantly increased compared to littermate controls (Figure $39 \mathrm{~A}$ ). This raise was likely due to an increase in K48 specific ubiquitination, as, measured by specific antibodies, there was a significant increase in K48 specific poly-ubiquitin levels while the levels of K63-linked poly-ubiquitin were unchanged (Figure $39 \mathrm{~A}$ ). It has previously been shown that total polyubiquitin levels are up-regulated in endophilin TKO brains (Cao, Milosevic, Giovedi, \& De Camilli, 2014). In addition, in endophilin TKO brain homogenates K48specific poly-ubiquitin was increased significantly, while at the same time, K63 polyubiquitin levels were normal (Figure $39 \mathrm{~B}$ ). This shows that endophilin mutants have an increase of proteins being marked for proteasomal degradation.
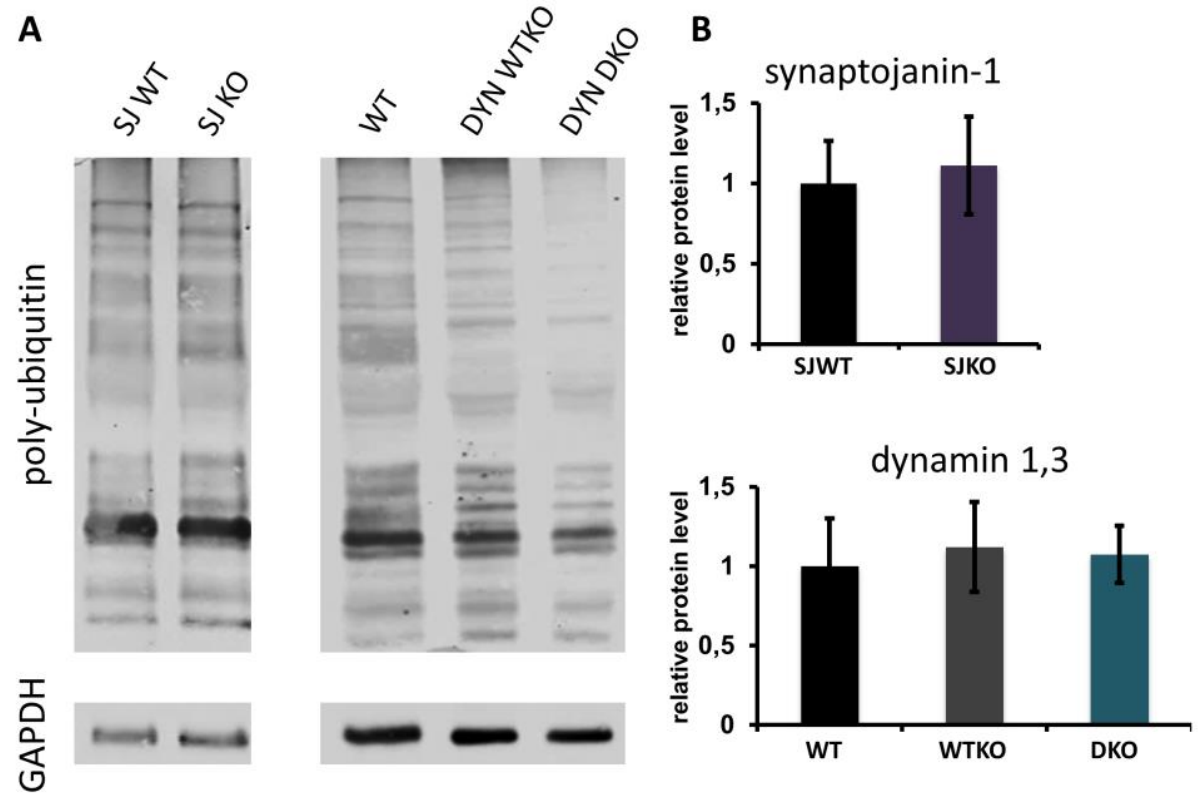

Figure 40 - Poly-ubiquitination is unaffected in synaptojanin and dynamin mutants. (A) Relative protein levels (normalized to GAPDH) of total poly-ubiquitin in p0 WT and synaptojanin-1 KO (left) and WT, dynamin 1WT-3KO and dynamin 1,3 DKO (right) brain homogenates were assessed by western blot analysis. (B) Quantification of protein levels in (A) Graphs represent mean \pm SEM. No significant differences were detected by two-sided Student's t-test. 
Synaptojanin-1 KO and dynamin 1,3 DKO brains were collected at $\mathrm{p} 0$ to test whether this finding is also observed in other endocytic mutants. Interestingly, in both mouse models, there was no detectable increase of poly-ubiquitination (Figure 40). This indicates that these endocytic mutants do not have an increase in E3 dependent protein-ubiquitination or that their UPS is capable of compensating for such an increase.

\subsubsection{Autophagy markers are dysregulated in endophilin mutants}

Given the proposed role of $\mathrm{Fbxo32}$ as a regulator between protein degradation through the UPS and the autophagy system (Zaglia, et al., 2014; see also chapter 1.6.3), several autophagy markers have been investigated in endophilin DKO and TKO brains (Murdoch, et al., 2016). This includes the early autophagy marker Atg5, which plays a role in the formation of the autophagophore (see chapter 1.5.2) and

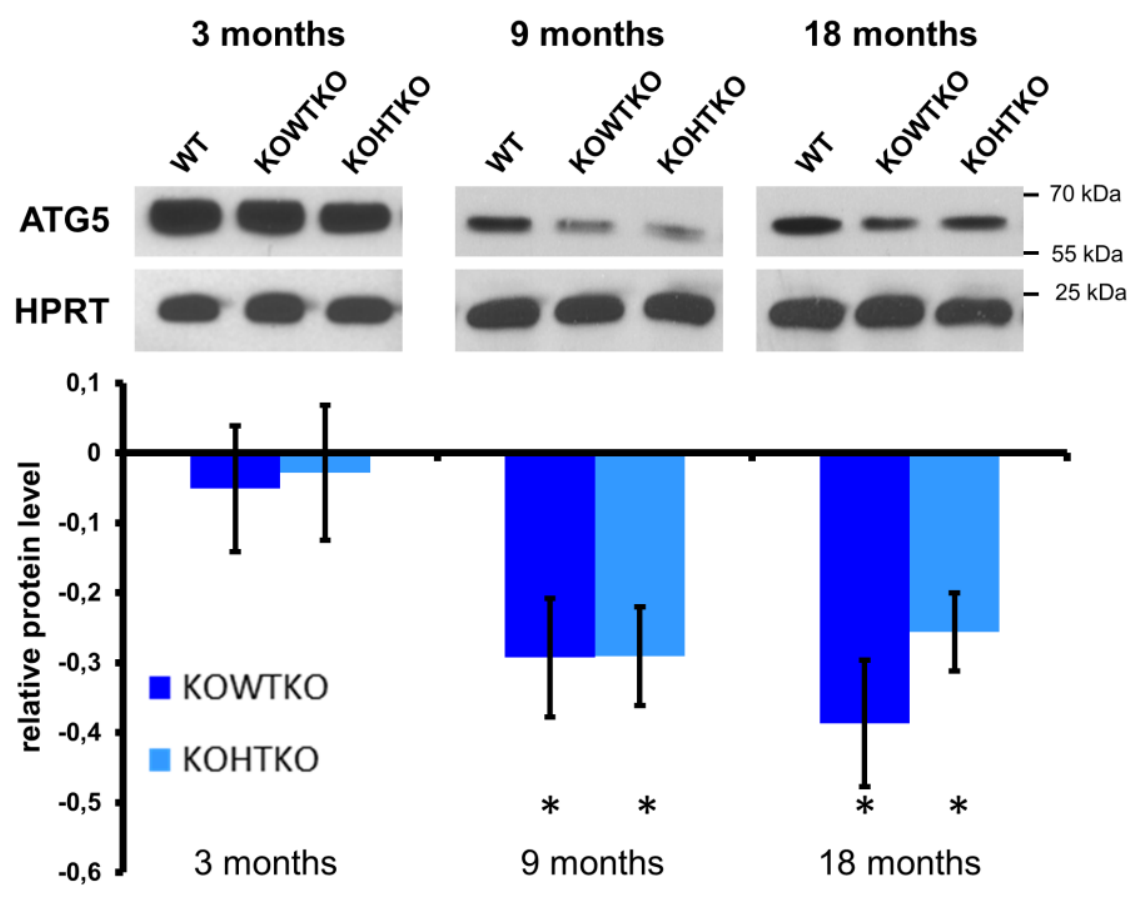

Figure 41 - Atg5 protein levels are reduced in aged endophilin 1KO-2HT-3KO brains. Atg5 levels relative to HPRT loading control were assessed by western blot at 3, 9 and 18 months. Atg5 was down-regulated in 9 and 18-month-old endophilin $1 \mathrm{KO}-2 \mathrm{HT}-3 \mathrm{KO}$ mice. Graphs represent mean fold change vs WT \pm SEM. ${ }^{*}=p<0.05$ by two-sided Student's t-test. 
was found to be significantly down-regulated in both endophilin TKO and DKO animals (Murdoch, et al., 2016).

To understand if autophagy is also affected in the model of aged endophilin 1KO2HT-3KO mice used for the motor behavior tests (see chapter 3.2) brain homogenates from these animals were analyzed by western blot. Biochemical analysis showed that while Atg5 levels were normal at 3 months of age, they were significantly reduced at the ages of 9 and 18 months in endophilin $1 \mathrm{KO}-2 \mathrm{HT}-3 \mathrm{KO}$ brains (Figure 41).

Two other autophagy factors, Atg9a and Atg16I, have been reported to be trafficked through CME (Ravikumar, Moreau, Jahreiss, Puri, \& Rubinsztein, 2010; Puri, Renna, Bento, Moreau, \& Rubinsztein, 2014). As they are part of the machinery that forms the autophagosome, their availability during the process might be a crucial factor for effective autophagy. As endophilin plays a key role in CME, it is possible that defective endocytosis of these two factors could result in impaired autophagy.

However, testing for protein levels of both autophagy factors showed that they were not changed in endophilin TKO (Figure $42 \mathrm{~A}$ ) or endophilin DKO brain homogenates (Figure $42 \mathrm{~B}$ ). This indicates that both factors are available at normal levels in endophilin mutants and are therefore unlikely to be the cause of autophagy impairments in endophilin deficient brains.

Interestingly, the other family member of the endophilin family, endophilin $B$, has previously been reported to play a role in autophagy (reviewed in Kjaerulff, Brodin, \& Jung, 2011; see also chapter 1.3.1). Given the structural similarity of endophilins A and $B$, it is possible that endophilin $A$ might play a similar role in autophagy as its family member endophilin B. However, endophilin B1 protein levels were not changed in endophilin A TKO brain homogenates (Figure $42 \mathrm{C}$ ). This indicates that in case endophilin A does play a role in autophagy, endophilin $B$ is not able to compensate for its loss in endophilin TKO brains.

To confirm that defective endocytosis is not the cause of the low levels of autophagy markers, other endocytic mutants were also tested for Atg5 protein levels. When 
A

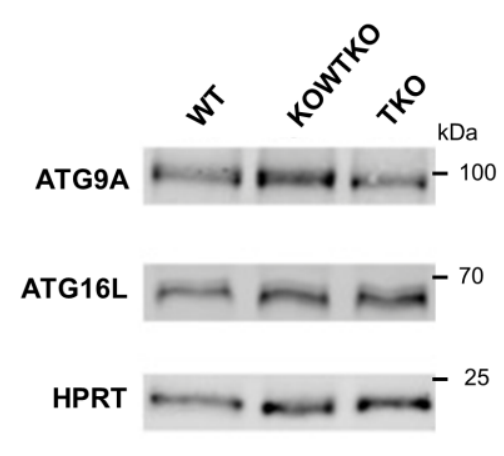

B

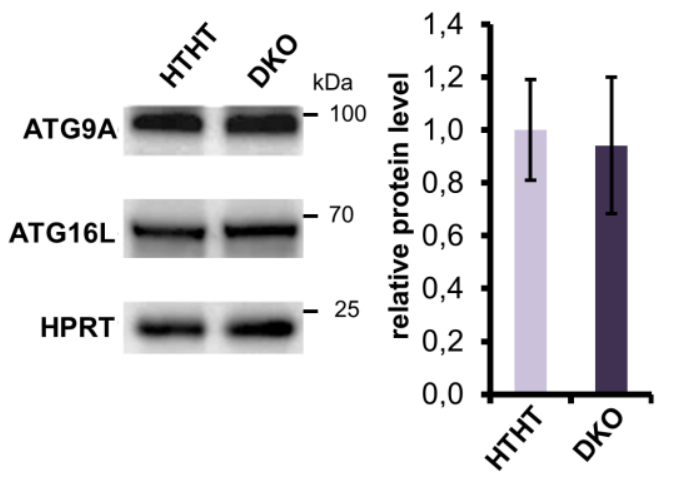

ATG9A

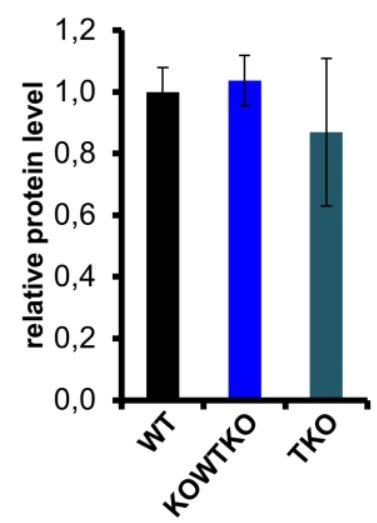

ATG16L

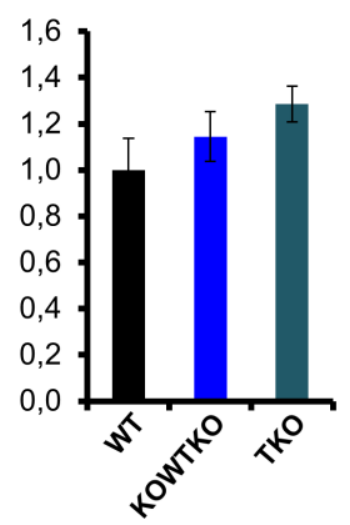

C

endoB1

ATG16L
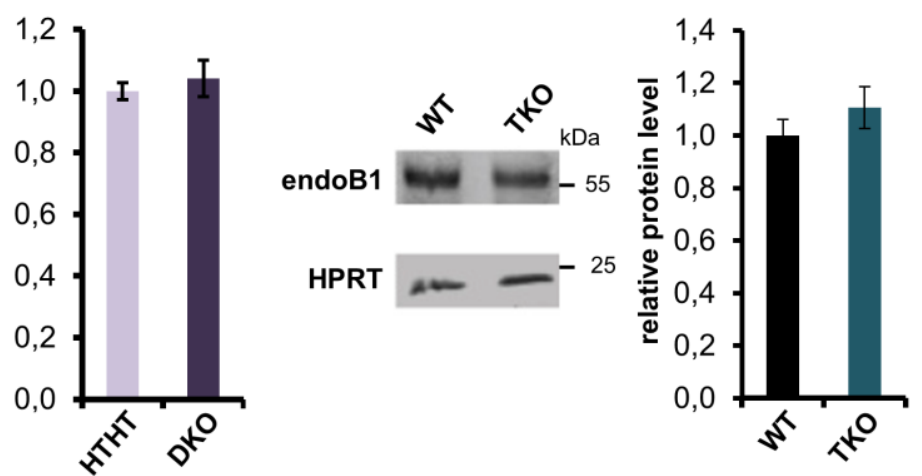

Figure 42 - Levels of Atg9a and Atg16I are unchanged in endophilin mutants. (A) Relative protein levels (normalized to HPRT) of Atg9a and Atg16l of p0 WT, endophilin 1KO-2WT-3KO and endophilin TKO brain homogenates. (B) Relative protein levels (normalized to HPRT) of Atg9a and Atg16l of p18 endophilin 1HT-2HT3WT and endophilin DKO brain homogenates. Part of the western blots for this figure were done by Dr. Amandeep Arora (ENI, Göttingen). (C) Relative protein levels (normalized to HPRT) of endophilin B1 of p0 WT, endophilin 1KO-2WT-3KO and endophilin TKO brain homogenates. Graphs represent mean \pm SEM. No significant changes were detected by two-sided Student's t-test.

testing synaptojanin-1 KO and dynamin 1,3 DKO mouse brain homogenates by western blot, they revealed no change in Atg5 levels in these animals (Figure 43). This provides further evidence that the loss of endophilin does not indirectly result in the autophagy impairment by abolishing endocytosis of important autophagy factors but suggests a more direct role of endophilin in this process.

In summary, biochemical analysis of endocytic mutants showed that the up-regulation of the E3-ubiquitin ligase Fbxo32 is a common feature of all endophilin mutants 
A

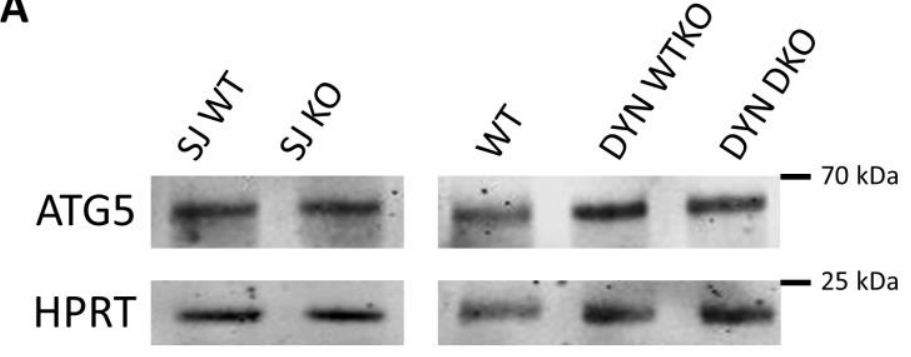

B synaptojanin-1

dynamin 1,3
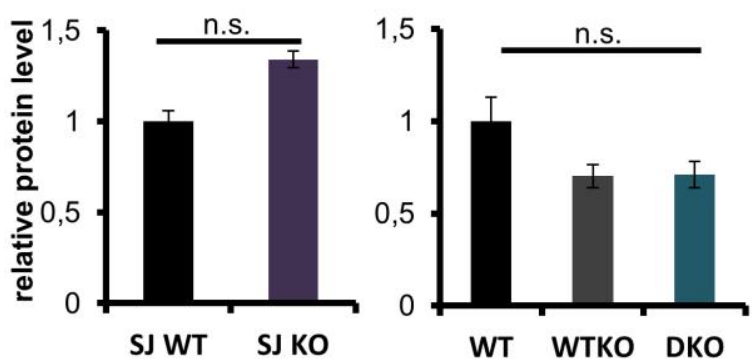

Figure 43 - Atg5 protein levels are unchanged in synaptojanin and dynamin mutants. (A) Relative protein levels (normalized to HPRT) of Atg5 in p0 WT and synaptojanin-1 KO (left) and WT, dynamin 1WT-3KO and dynamin 1,3 DKO (right) brain homogenates were assessed by western blot analysis. (B) Quantification of protein levels in (A) Graphs represent mean \pm SEM. No significant differences were detected by two-sided Student's t-test.

tested. In the same mutants, protein homeostasis seems to be impaired as there is an increase of poly-ubiquitinated proteins that are marked for UPS degradation accompanied by a decrease in the early autophagy marker Atg5. The impaired proteostasis however, is likely not caused by defective endocytosis as two autophagy factors, that have been reported to undergo CME, are present at normal levels in endophilin mutants and because other endocytic mutants do not display the same phenotype. This points to a direct role of endophilin in the process of autophagy. 


\subsection{Rescue of motor impairments in endophilin mutants by genetic reduction of $\mathrm{Fbx032}$}

\subsubsection{Fbxo32 co-localizes with endophilin on SV necks}

To further understand the connection between Fbxo32 and endophilin, Fbxo32 localization was assessed in a cell model of arrested endocytosis. Specifically,

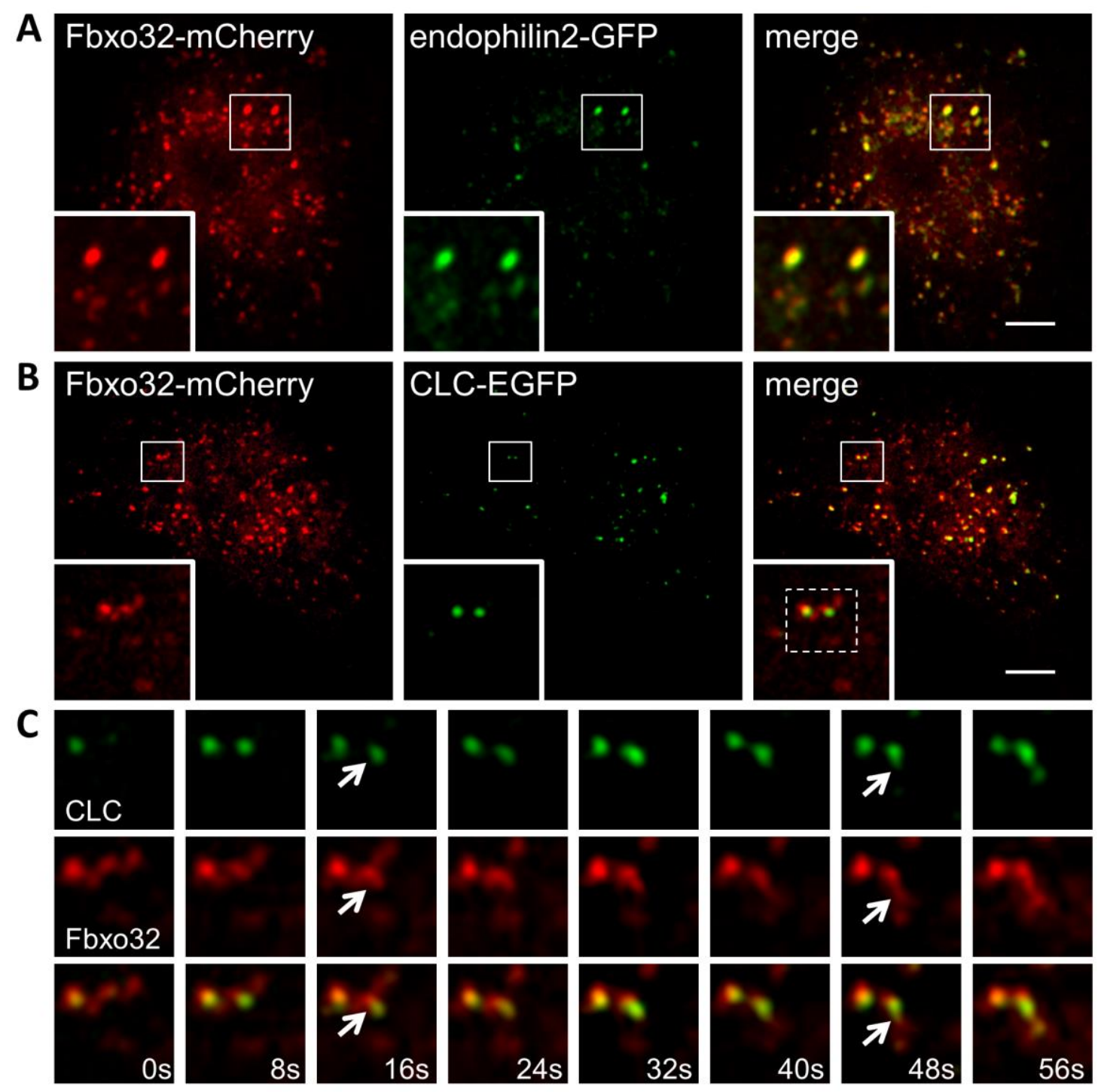

Figure 44 - Fbxo32 localizes to SV necks during endocytosis. Spinning disk live cell imaging of dynamin TKO MEFs transfected with Fbxo32-mCherry and either endophilin2-GFP (A) or clathrin-light-chain (CLC)-EGFP (B-C). (C) Time lapse of the dashed insert in (B). On arrested endocytic pits, Fbxo32 co-localizes with endophilin 2 and juxtaposed to CLC. Scale bar $7 \mu \mathrm{m}$. 
dynamin TKO MEFs were cultured (Ferguson, et al., 2009; Rock, et al., 1994) and transfected with Fbxo32 with either endophilin 2 or clathrin light chain (CLC). In this model, dynamin dependent scission of the SV from the plasma membrane is completely abolished. As a result, stable clathrin-coated pits (CCPs) are enriched that have elongated necks. In these cells, Fbxo32 co-localized prominently with endophilin 2 (Figure $44 \mathrm{~A}$ ) and was located juxtaposed to CLC (Figure $44 \mathrm{~B}$ ). Interestingly, in cotransfections of Fbxo32 with CLC, Fbxo32 was observed to be enriched on long tubular structures "crowned" with CLC signal (Figure $44 \mathrm{C}$ ).

As these data imply that Fbxo32 might play a role in endocytosis, endophilin levels were assessed in a mouse model that carries a genetic deletion of Fbxo32, kindly provided by Prof. Marco Sandri. These mice have a normal phenotype when they are young but develop muscle atrophy at the age of 16 months (Bodine, et al., 2001; Zaglia, et al., 2014). Levels of all three endophilins were unchanged in p0 brains collected from Fbxo32 HT and KO mice (Figure 45). Though that does not rule out a

A

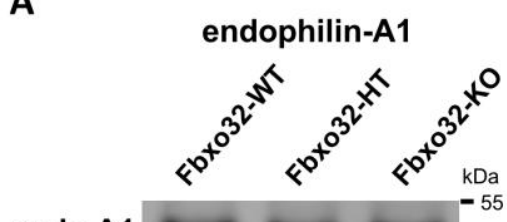

endo $\mathbf{A} 1$
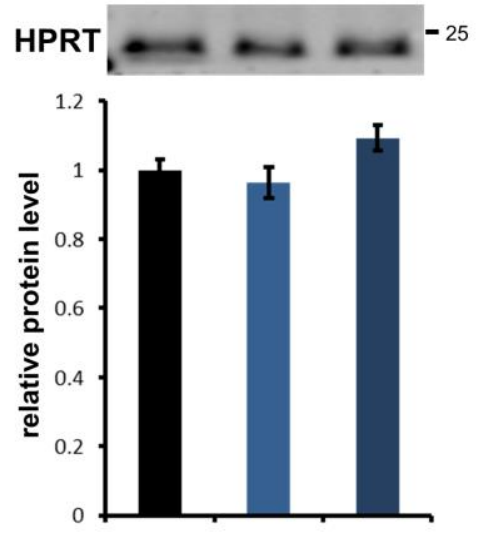

B
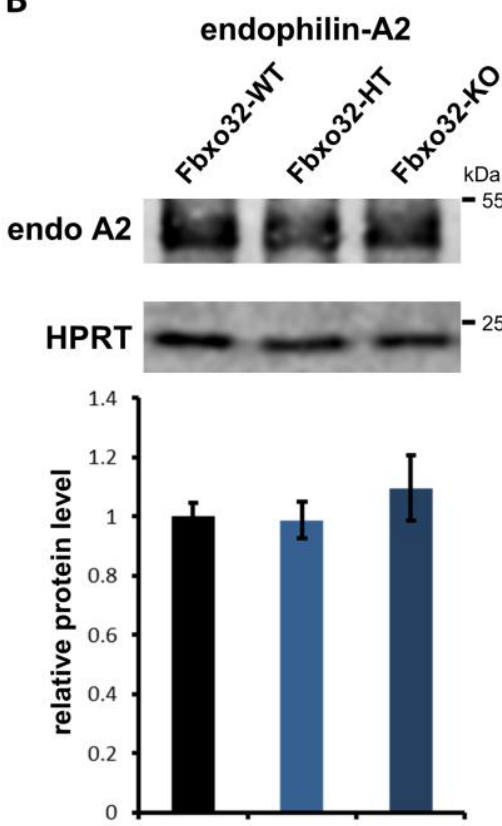

C
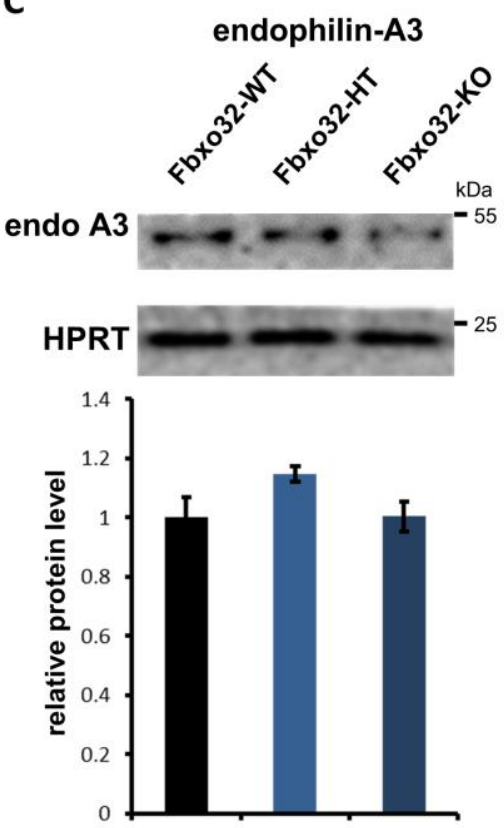

Figure 45 - Endophilin levels are normal in Fbxo32 KO brains. Relative protein levels (normalized to HPRT) of endophilin 1 (A), endophilin 2 (B) and endophilin 3 (C) in p0 WT, Fbxo32 HT and Fbxo32 KO brain homogenates was assessed by western blot analysis. (bottom) Quantification of protein levels. Graphs represent mean \pm SEM. No significant differences were detected by two-sided Student's t-test. 
role of Fbxo32 in endocytosis, it shows that at $\mathrm{p} 0$ endophilin influences the levels of Fbxo32 (Figure 33 and Murdoch, et al., 2016) but not vice versa.

\subsubsection{Genetic reduction of Fbxo32 does not affect grip strength in endophilin mutants}

In order to elucidate if the observed motor impairments and the mild cell death in the brains of various endophilin mutants could be a result of high Fbxo32 levels, endophilin deficient mice were crossed with the Fbxo32 KO mouse line. As NGS and qPCR analysis showed a 2-fold increase of Fbxo32 (Murdoch, et al., 2016), the mutants were crossed in such a way, that the offspring had $50 \%$ gene expression of Fbxo32 left. Genetic reduction of Fbxo32 was confirmed by genotyping (Figure 46). The systemic consequences of this reduction were then assessed by motor behavior tests.

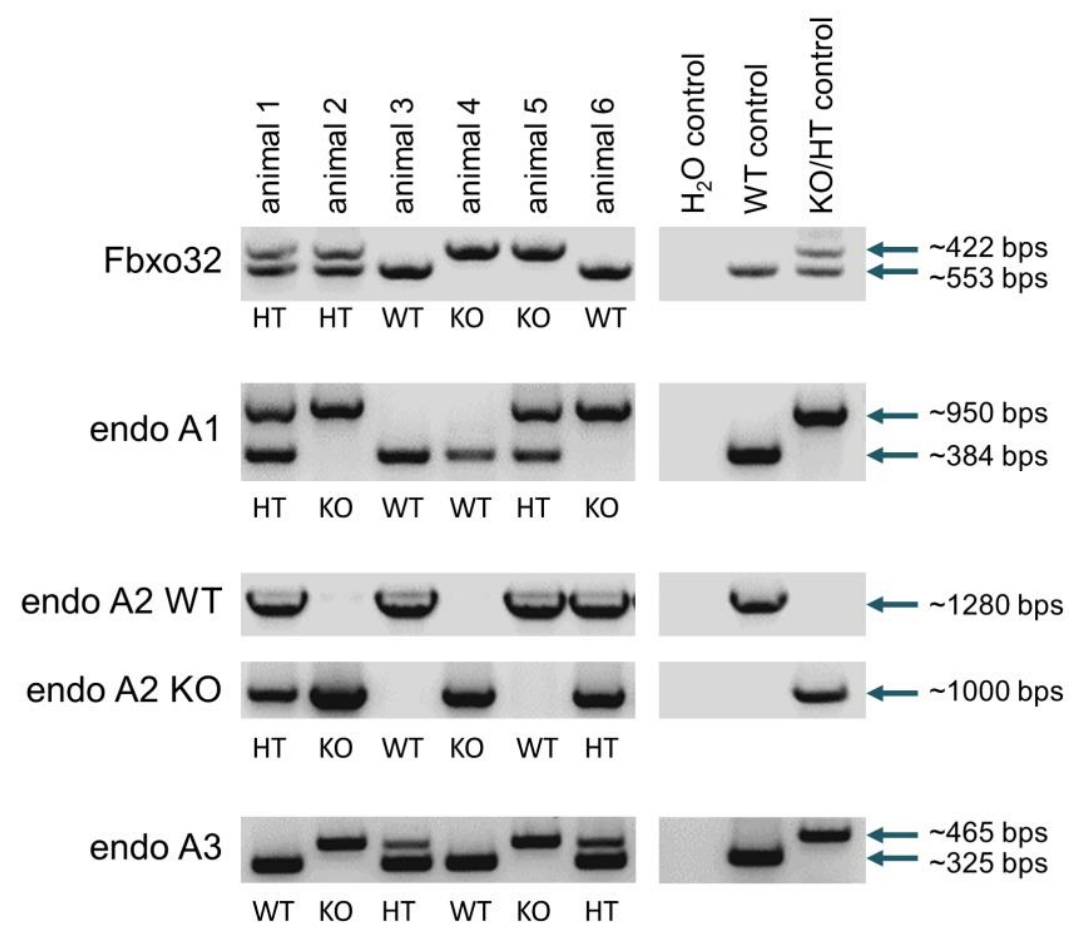

Figure 46 - Genotyping for Fbxo32 deficient endophilin mutants. Offspring of Fbxo32 KO mice crossed with endophilin 1KO-2HT-3KO mice was tested by PCRs for Fbxo32, endophilin 1, endophilin 2 (WT and KO reaction separated) and endophilin 3. Genetic full deletion (KO) or heterozygous (HT) deletion of Fbxo32 was confirmed by this method. 
Chapter 3.2 of this thesis illustrated motor deficits that occur in several endophilin mutants depending on how many endophilin alleles were deleted and the age of the test animals. These deficits could be a result of defective endocytosis. However, other tested endocytic mutants showed no or only mild motor impairments (chapter 3.3). Given that endophilin mutants overexpress Fbxo32 and seem to have a dysregulation of autophagy (chapter 3.5), it is also possible that problems in protein degradation contribute to the phenotype.

In that case, genetic reduction of Fbxo32 levels could potentially ameliorate the observed motor behavior deficits. For this reason, animals that only have one Fbxo32 allele (Fbxo32 HT) and were either endophilin 1KO-2WT-3WT, 1KO-2HT-3WT or $1 \mathrm{KO}-2 \mathrm{HT}-3 \mathrm{KO}$ were tested for their motoric abilities with the same tests used in chapter 3.2. The results from endophilin 1KO-2HT-3KO with WT and HT Fbxo32 levels are shown in this section.

A

\begin{tabular}{|c|c|c|c|c|c|}
\hline \multirow{3}{*}{$\begin{array}{l}\rightarrow-\text { F32KO-E1WT-E2WT-E3WT } \\
\text { - F32WT-E1KO-E2HT-E3KO }\end{array}$} & \multicolumn{5}{|c|}{ v.s. -o- F32WI-E1WT-E2WI-E3WI } \\
\hline & n.s. & n.s. & n.s. & n.s. & n.s. \\
\hline & $\mathrm{n} . \mathrm{s}$ & $\mathrm{n} . \mathrm{s}$ & n.s. & $\mathrm{n} . \mathrm{s}$ & $*$ \\
\hline
\end{tabular}

$\rightarrow$ F32HT-E1KO-E2HT-E3KO

\begin{tabular}{|c|c|c|c|c|}
\hline \multicolumn{4}{|c|}{ v.s. -} & F32WT-E1KO-E2HT-E3KO \\
\hline$*$ & n.s. & $* * *$ & n.s. & n.s. \\
\hline
\end{tabular}

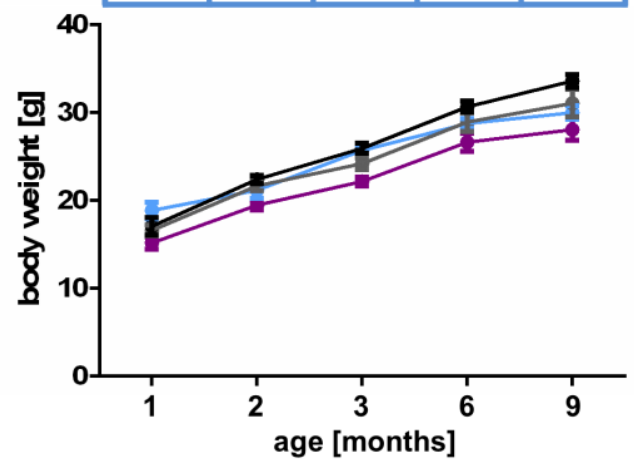

B normalized grip strength

\begin{tabular}{|c|c|c|c|c|}
\hline v.s. $\rightarrow-$ & F32WT-E1WT-E2WT-E3WT \\
\hline$*$ & n.s. & n.s. & n.s. & n.s. \\
\hline n.s. & n.s. & $* *$ & $*$ & $*$ \\
\hline
\end{tabular}
V.S. - - F32WT-E1KO-E2HT-E3KO \begin{tabular}{|l|l|l|l|l}
$* *$ & $*$ & n.s. & n.s. & n.s. \\
\hline
\end{tabular}

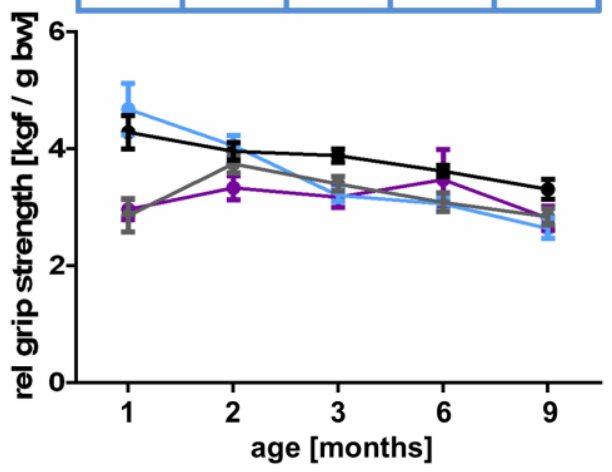

Figure 47 - Weight and grip strength are decreased in Fbxo32 deficient endophilin 1KO-2HT-3KO mice. (A) Body weight and (B) normalized grip strength of BL6 WT (black), Fbxo32 KO (grey), Fbxo32 WT-endophilin 1KO-2HT-3KO (blue) and Fbxo32 HT-endophilin 1KO-2HT-3KO (light blue) animals. Graphs show mean \pm SEM. Significance by Dunnett- (v.s WT) or Tukey-corrected (vs. endophilin $1 \mathrm{KO}-2 \mathrm{HT}-3 \mathrm{KO}$ ) one-way ANOVA is shown in the tables above the chart. ${ }^{* *}=\mathrm{p}<0.001$; ${ }^{* *}=p<0.01 ;{ }^{*}=p<0.05 . \mathrm{kgf}=$ kilogram force 
The reduction of $\mathrm{Fbx032}$ affected the body weight, though the body weight was reduced in Fbxo32 HT-endophilin 1KO-2HT-3KO mutants only at a young age and no longer at 6 and 9 months of age (Figure $47 \mathrm{~A}$ and Supplemental Figure 2). In addition, the grip strength was lower in these animals, especially in young Fbxo32 HTendophilin 1KO-2HT-3KO mice (Figure $47 \mathrm{~B}-\mathrm{C}$ and Supplemental Figure 2). This reduction in grip strength might be an early indicator for the development of muscle atrophy at the age of 16 months which has been reported for Fbxo32 KO mice (Bodine, et al., 2001; Zaglia, et al., 2014).

\subsubsection{Rescue of motor coordination deficits in endophilin mutants by genetic reduction of $\mathrm{Fbx032}$}

When motor coordination in these animals was assessed by Rotarod testing, genetic reduction of Fbxo32 seemed to be beneficial. As described before, endophilin 1KO-

A

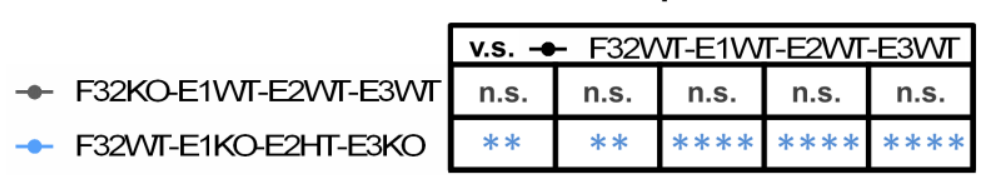

fixed-speed Rotarod

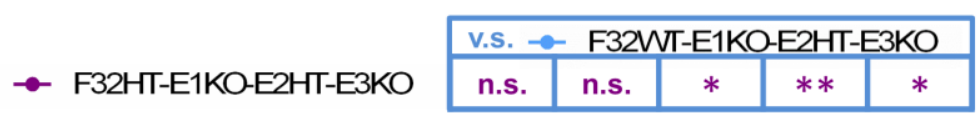

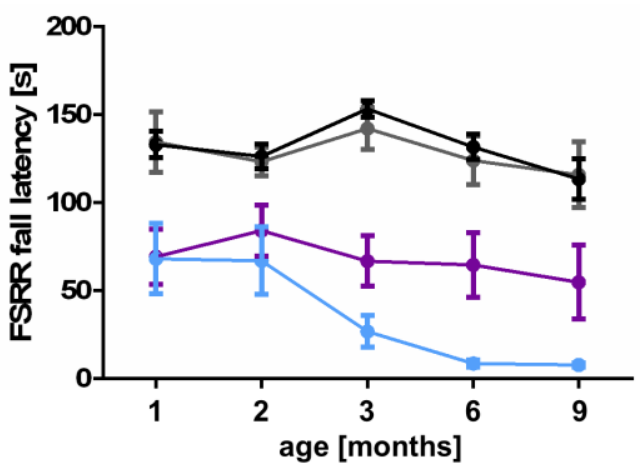

B
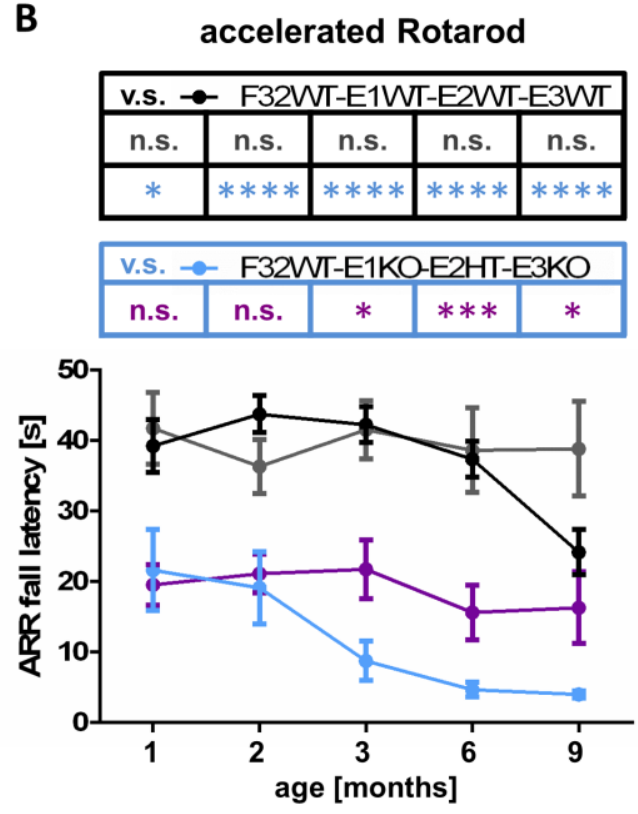

Figure 48 - Rotarod performance is ameliorated upon genetic reduction of Fbx032 in endophilin 1KO-2HT-3KO mice. (A) Fall latency from the fixed speed Rotarod and (B) accelerated Rotarod of BL6 WT (black), Fbxo32 KO (grey), Fbxo32 WT-endophilin 1KO-2HT-3KO (blue) and Fbxo32 HT-endophilin 1KO-2HT-3KO (light blue) animals. Graphs show mean \pm SEM. Significance by Dunnett- (v.s WT) or Tukeycorrected (vs. endophilin 1KO-2HT-3KO) one-way ANOVA is shown in the tables above the chart. ${ }^{* * *}=p<0.0001 ;{ }^{* *}=p<0.01 ;{ }^{*}=p<0.05$. 
2HT-3KO mice fell significantly earlier from the Rotarod in both the training and the testing phase (Figure 24). This motor deficit was apparent already at an age of 1 month and deteriorated with aging. Interestingly, Fbxo32 HT-endophilin 1KO-2HT$3 \mathrm{KO}$ mice performed significantly better in this task, though they did not perform as well as WT animals (Figure 48). In endophilin 1KO-2WT-3WT and 1KO-2HT-3WT mice, partial deletion of Fbxo32 also trended to increase Rotarod performance, though the difference was not significant (Supplemental Figure 3).

Fbxo32 deficient endophilin mutants were also tested on the DigiGait treadmill. The average running speed was mostly unaffected by Fbxo32 reduction (Figure 49 and Supplemental Figure 4), but it did decrease the running speed of endophilin 1KO2HT-3WT animals at 6 and 9 months (Supplemental Figure 4). On the other hand side, the genetic reduction of Fbxo32 trended to improve the average running speed of 9-month-old endophilin $1 \mathrm{KO}-2 \mathrm{HT}-3 \mathrm{KO}$ mice though the difference was not significant (Figure 49).

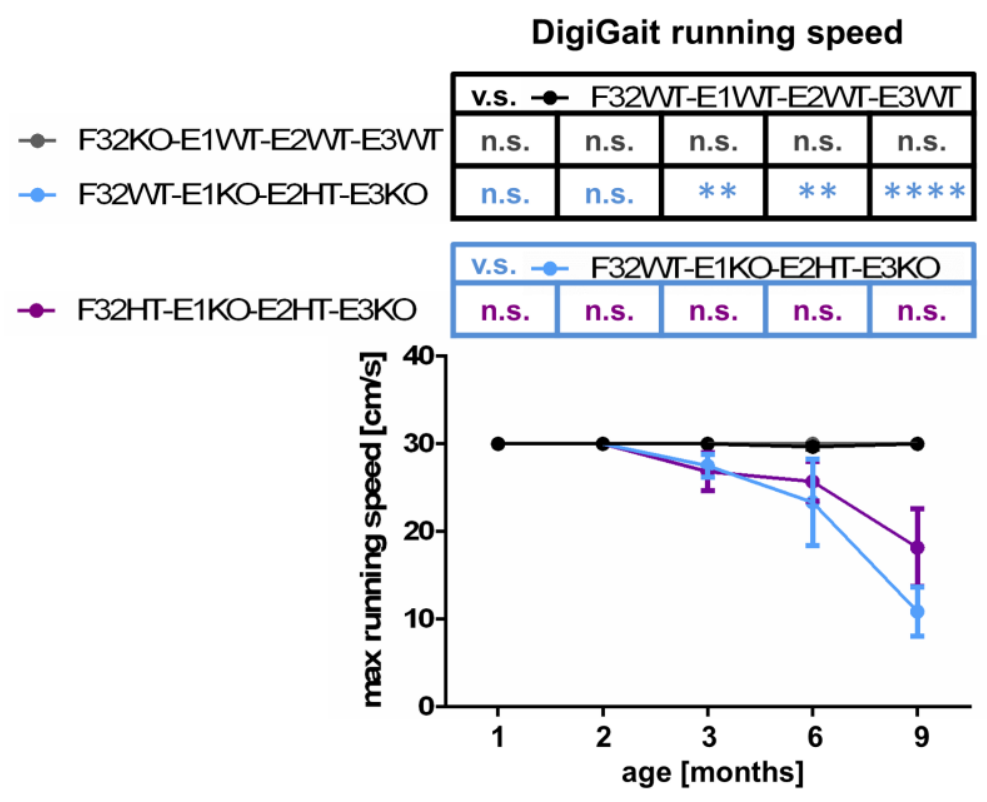

Figure 49 - Maximal running speed of Fbxo32 deficient endophilin 1KO-2HT$3 \mathrm{KO}$ mice. Average running speed of BL6 WT (black), Fbxo32 KO (grey), Fbxo32 WT-endophilin 1KO-2HT-3KO (blue) and Fbxo32 HT-endophilin 1KO-2HT-3KO (light blue) animals. Graphs show mean \pm SEM. Significance by Dunnett- (v.s WT) or Tukeycorrected (vs. endophilin 1KO-2HT-3KO) one-way ANOVA is shown in the tables above the chart. ${ }^{* * *}=p<0.0001 ;{ }^{* *}=p<0.01$. 


\subsubsection{Genetic reduction of Fbxo32 has no additional effect on gait rhythm in endophilin mutants}

As before, gait rhythm was assessed by measuring timing of the paws in air and on the ground. Fore limb stance and swing time were not affected by genetic deletion of Fbxo32 in any of the tested endophilin mutants (Figure $50 \mathrm{~A}$ ), though it trended to increase swing time in all one-month-old endophilin mutants (Figure $50 \mathrm{~B}$ and Supplemental Figure 5). This trend was no longer observed at 3 months. The same parameters were found to be unchanged in the hind limbs of endophilin mutants (Supplemental Figure 7). However, genetic reduction of Fbxo32 trended to increase the swing time of endophilin 1KO-2WT-3WT and 1KO-2HT-3WT mice (Supplemental Figure $7 \mathrm{~B}$ ). The trend was only significant in 6-month-old Fbxo32 HT-endophilin 1KO2WT-3WT animals. Overall, the gait rhythm of both fore and hind limbs of endophilin $1 \mathrm{KO}-2 \mathrm{HT}-3 \mathrm{KO}$ mice was not changed upon genetic reduction of Fbxo32.

During stance, the timing of brake and propel phase was not changed in the fore limbs of enophilin 1KO-2HT-3KO animals that are heterozygous for Fbxo32 (Figure $50 \mathrm{C}$ ). Only in young Fbxo32 HT-endophilin 1KO-2WT-3WT and Fbxo32 HT-endophilin $1 \mathrm{KO}-2 \mathrm{HT}-3 \mathrm{WT}$, the propel time decreased (Supplemental Figure $6 \mathrm{~A}$ ) while at the same time the brake time trended up (Supplemental Figure $6 \mathrm{~B}$ ). This was no longer the case from the age of 3 months onwards. Taken together, fore limb gait rhythm is only impacted in a minor way after additional deletion of Fbxo32 in endophilin mutants. The observed changes, if at all, only occur at a young age and are no longer observed after the age of 2 months.

Again, in the hind limbs of the same animals, Fbxo32 reduction had little impact on the brake / propel timing. Only 2-month-old Fbxo32 HT-endophilin 1KO-2HT-3WT had a significantly longer brake time when compared to Fbxo32 WT-endophilin 1KO-2HT3WT mice (Supplemental Figure 8A). This change was no longer present in older animals and was not observed in any of the other tested endophilin mutants. Congruously, propel time was not further affected in any of the analyzed Fbxo32 deficient endophilin mutants (Supplemental Figure 8B). 
A

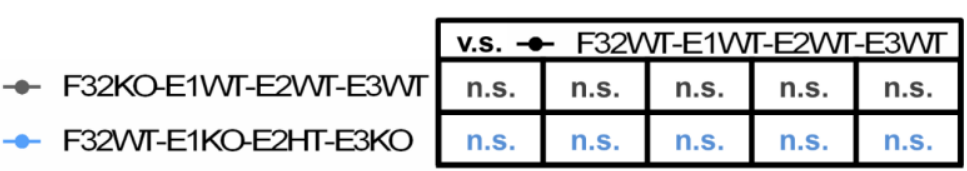

F32HT-E1KO-E2HT-E3KO

stance time

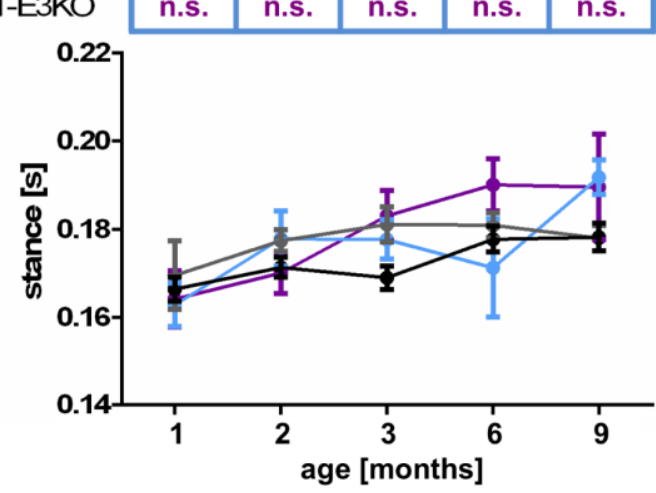

C

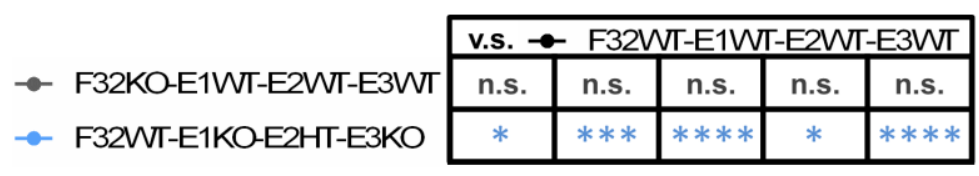

F32HT-E1KO-E2HT-E3KO
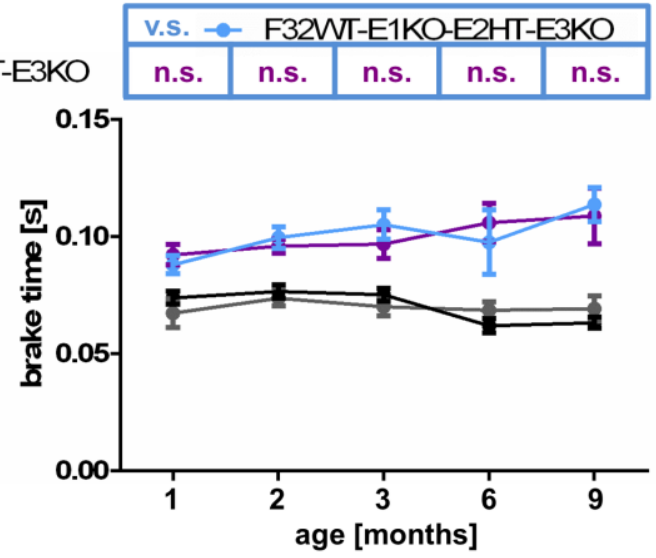

B

swing time
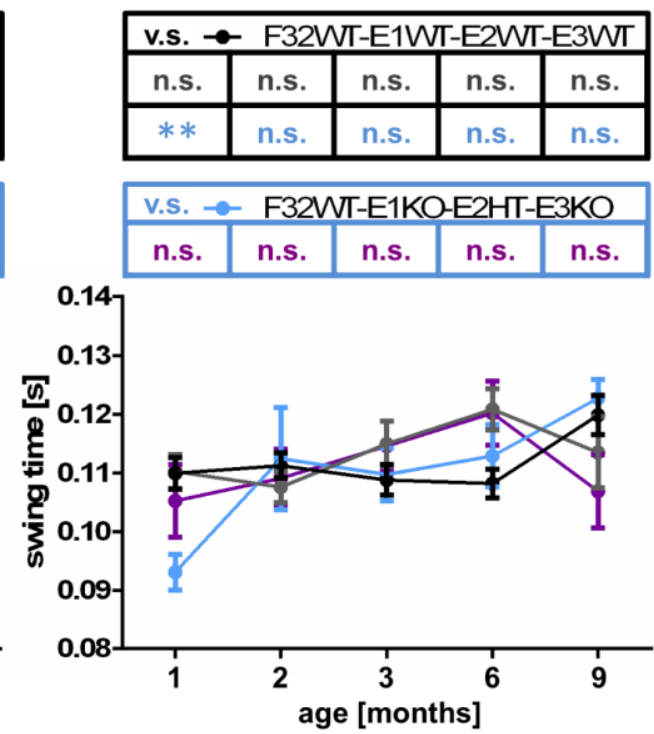

D

propel time

\begin{tabular}{|c|c|c|c|c|}
\hline \multicolumn{5}{|c|}{ v.s. $-\mathrm{F} 32 \mathrm{WT}-\mathrm{E} 1 \mathrm{WT}-\mathrm{E} 2 \mathrm{WT}-\mathrm{E} 3 \mathrm{WT}$} \\
\hline n.s. & n.s. & $*$ & n.s. & n.s. \\
\hline n.s. & $* *$ & $*$ & $*$ & $* * * *$ \\
\hline
\end{tabular}
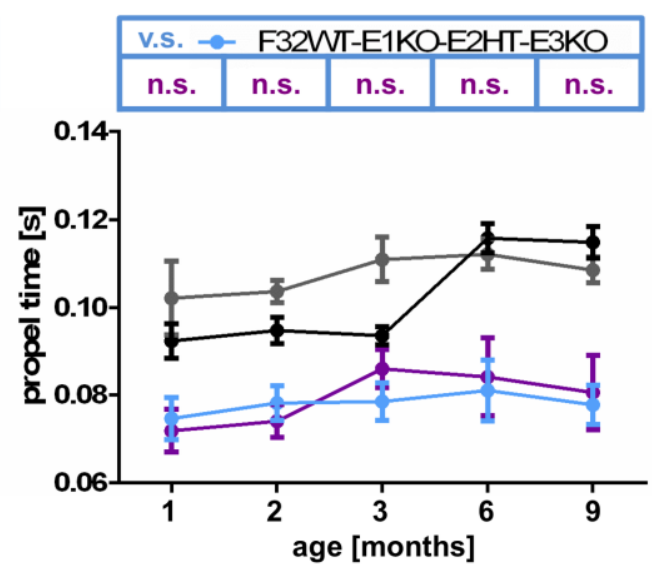

Figure 50 - Fore limb gait rhythm is not affected in Fbxo32 deficient endophilin 1KO-2HT-3KO mice. Gait timing parameters of BL6 WT (black), Fbxo32 KO (grey), Fbxo32 WT-endophilin 1KO-2HT-3KO (blue) and Fbxo32 HT-endophilin 1KO-2HT$3 \mathrm{KO}$ (light blue) animals. (A) Stance time = time the paw is in contact with the ground. (B) Swing time = time the paw is in the air. (C) Brake time = during stance, the time that the paw area is increasing. (D) Propel time = during stance, the time that the paw area is decreasing. Running speed $=20 \mathrm{~cm} / \mathrm{s}$. Graphs show mean $\pm S E M$. Significance by Dunnett- (v.s WT) or Tukey-corrected (vs. endophilin 1KO-2HT-3KO) one-way ANOVA is shown in the tables above the chart. ${ }^{* * *}=p<0.0001 ;{ }^{* * *}=p<0.001$; ${ }^{* *}=p<0.01 ;{ }^{*}=p<0.05$. 


\subsubsection{Posture is ameliorated in endophilin mutants after genetic reduction of Fbxo32}

When looking into the posture of the different endophilin mutants, DigiGait analysis showed that the paw angle was reduced in all tested endophilin deficient groups, even though the change was not significant at all ages (compare Figure $27 \mathrm{~A}$ ). Genetic reduction of Fbxo32 in the same mutants seemed to inverse the observed effect. The trend was present in all tested groups in the age of 1-6 months and was significant in 2 months old Fbxo32 HT-endophilin 1KO-2HT-3WT mice (Supplemental Figure $9 \mathrm{~A}$ ) and 3- and 6-month-old Fbxo32 HT-endophilin 1KO-2HT-3KO animals (Figure $51 \mathrm{~A}$ ). However, the paw angle variability was not changed upon reduction of Fbxo32 levels (Figure 51 B and Supplemental Figure 9 B).

The positioning of the paws during running on the DigiGait treadmill was also unaffected by genetic reduction of Fbxo32 expression. While it did significantly decrease the overlap of ipsilateral fore and hind limbs of 3-month-old endophilin 1KO2HT-3WT mice (Supplemental Figure $10 \mathrm{~A}$ ), this parameter was unchanged in other Fbxo HT-endophilin mutants (Figure 51 D and Supplemental Figure 10 A). However, the percentage of shared stance, which was on average increased in endophilin mutants (compare Figure $27 \mathrm{C}$ ), trended to decrease in young endophilin 1KO-2WT3WT and 1KO-2HT-3WT mutants that are HT for Fbxo32 (Supplemental Figure 10 B). This decrease was significant in 1- and 2-month-old Fbxo32 HT-endophilin 1KO2WT-3WT mice. At a higher age however, Fbxo32 reduction had no influence on the percentage of shared stance during running and it also had no impact on endophilin 1KO-2HT-3KO mice.

Of note, full $\mathrm{KO}$ of Fbxo32 had very little effect on motor behavior. Fbxo32 KO mice trended to have increased fall latency from the Rotarod, yet the difference was never significant. In fact, the only significant changes by ANOVA analysis were a decreased grip strength at 1 month and an increase of fore limb propel time at the age of 3 months. Both changes, however, were no longer observed at higher ages. Posture parameters measured by DigiGait analysis were also found to be unchanged in Fbxo32 KO mice. 
A

\begin{tabular}{|c|c|c|c|c|c|}
\hline \multirow[b]{2}{*}{ - - F32KO-E1WT-E2WT-E3WT } & \multicolumn{5}{|c|}{ v.s. $\rightarrow$ F32WIT-E1WT-E2WI-E3WT } \\
\hline & n.s. & n.s. & n.s. & n.s. & n.s. \\
\hline - F32WT-E1KO-E2HT-E3KO & n.s. & n.s. & $* *$ & $* *$ & $* * * *$ \\
\hline & \multicolumn{5}{|c|}{ v.s. - $\rightarrow-$ F32WT-E1KO-E2HT-E3KO } \\
\hline 2HT-E1KC & n.s. & n.s. & $* * *$ & n.s. & $* * *$ \\
\hline
\end{tabular}

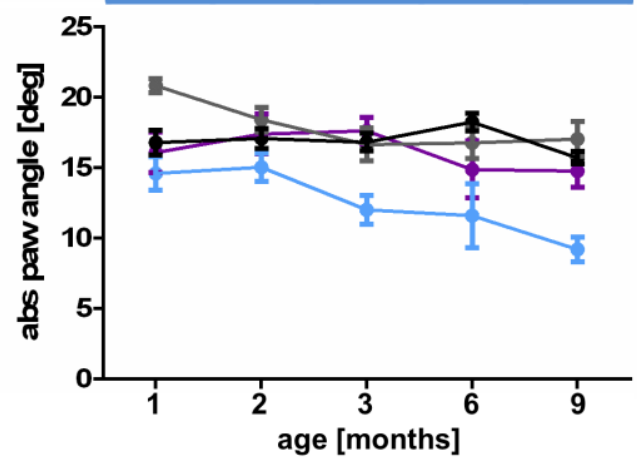

C

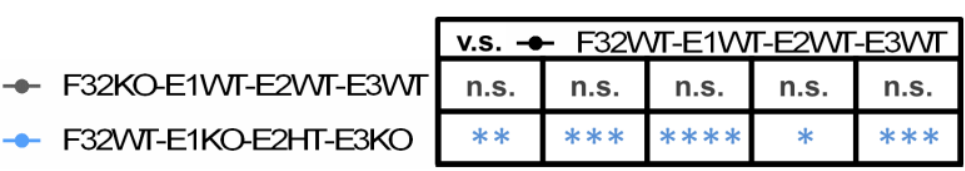

\section{F32HT-E1KO-E2HT-E3KO}

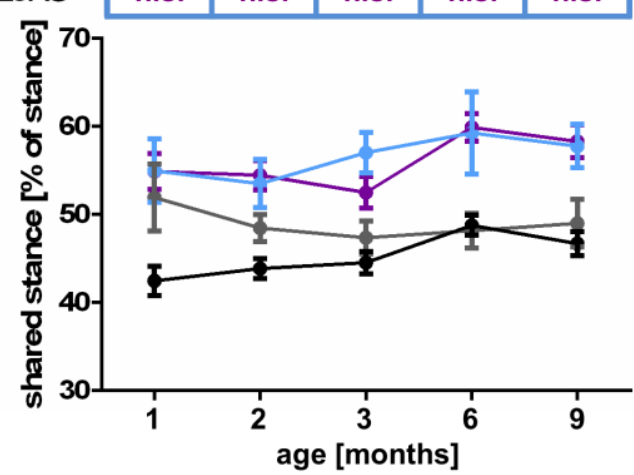

B

paw angle variability

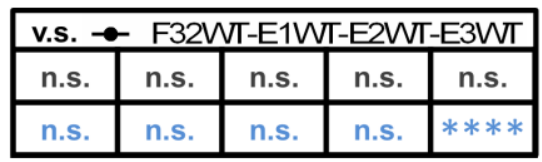

v.s. - - F32WT-E1KO-E2HT-E3KO \begin{tabular}{|l|l|l|l|l|}
\hline n.s. & n.s. & n.s. & n.s. & n.s. \\
\hline
\end{tabular}

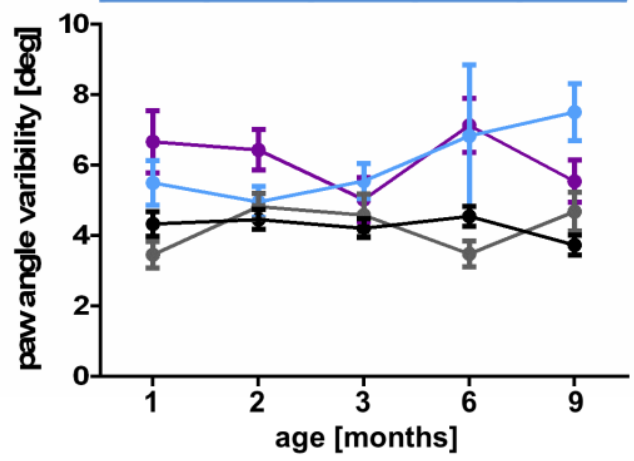

D

paw placement

\begin{tabular}{|c|c|c|c|c|}
\hline \multicolumn{4}{|c|}{ v.s. - F32WT-E1WT-E2WT-E3WT } \\
\hline n.s. & n.s. & n.s. & n.s. & n.s. \\
\hline n.s. & n.s. & n.s. & n.s. & $*$ \\
\hline
\end{tabular}
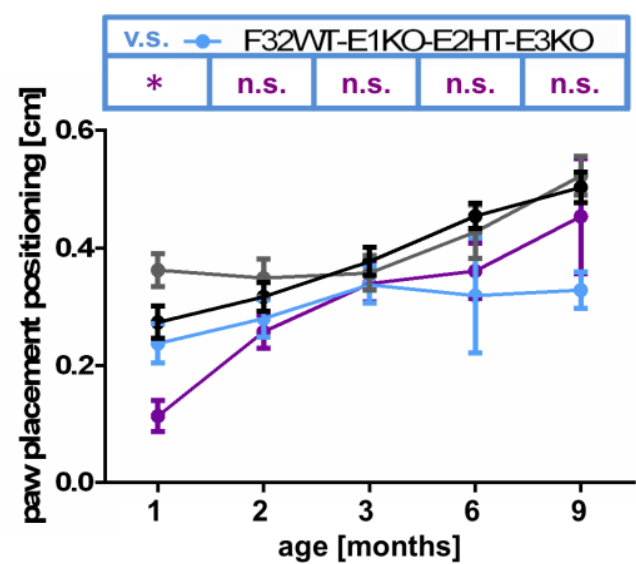

Figure 51 - Fbx032 deficiency in endophilin 1KO-2HT-3KO mice ameliorates posture during running. Hind limb posture parameters at $20 \mathrm{~cm} / \mathrm{s}$ of BL6 WT (black), Fbxo32 KO (grey), Fbxo32 WT-endophilin 1KO-2HT-3KO (blue) and Fbxo32 HTendophilin 1KO-2HT-3KO (light blue) animals. (A) Paw angle = angle between the paw and the snout-tail axis. (B) Paw angle variability = standard deviation of the paw angle. (C) Paw placement positioning = extent of overlap between ipsilateral fore and hind paws during full stance. (D) Shared stance = time both hind limbs are in contact with the ground at the same time. Graphs show mean \pm SEM. Significance by Dunnett(v.s WT) or Tukey-corrected (vs. endophilin 1KO-2HT-3KO) one-way ANOVA is shown in the tables above the chart. ${ }^{* * * *}=p<0.0001 ;{ }^{* * *}=p<0.001 ;{ }^{* *}=p<0.01$; ${ }^{*}=p<0.05$. 


\subsubsection{Comparison of motor impairments in endophilin mutants with and without genetic reduction of Fbxo32}

The changes in motor behavior of endophilin mutants with normal and reduced levels of Fbxo32 are summarized in Table 14. As reported in chapter 3.2, endophilin mutants displayed motor impairments depending on the age and the severity of the endophilin deletion. These changes of endophilin 1KO-2HT-3KO mice are summarized in column 3 of Table 14, while the additional effects of the 50\% Fbxo32 expression until the age of 9 months are summarized in column 4 .

Table 14 - Summary of motor behavior parameters in endophilin mutants with WT and $\mathrm{HT}$ levels of Fbxo32

\begin{tabular}{|c|c|c|c|}
\hline parameter & definition & $\begin{array}{c}\text { change in endophilin } \\
1 \mathrm{KO}-2 \mathrm{HT}-3 \mathrm{KO} \text { mice }\end{array}$ & $\begin{array}{c}\text { additional effect } \\
\text { of Fbxo32 } \\
\text { reduction }\end{array}$ \\
\hline \multicolumn{4}{|c|}{ Weight / muscle strength } \\
\hline Body weight & - & $\downarrow$ & $y$ \\
\hline $\begin{array}{l}\text { Max grip } \\
\text { strength }\end{array}$ & $\begin{array}{l}\text { Maximal grip strength } \\
\text { measured on the grip } \\
\text { strength meter }\end{array}$ & $\downarrow$ & $\downarrow$ \\
\hline $\begin{array}{l}\text { Normalized } \\
\text { grip strength }\end{array}$ & $\begin{array}{l}\text { Max grip strength / body } \\
\text { weight }\end{array}$ & $\downarrow$ & $\downarrow$ \\
\hline \multicolumn{4}{|c|}{ Motor coordination } \\
\hline FSRR & $\begin{array}{l}\text { Fall latency from the fixed } \\
\text { speed RotaRod }\end{array}$ & $\downarrow$ & 个 \\
\hline ARR & $\begin{array}{l}\text { Fall latency from the } \\
\text { accelerated RotaRod }\end{array}$ & $\downarrow$ & $\uparrow$ \\
\hline Running speed & $\begin{array}{l}\text { Maximal running speed at } \\
\text { which a video was recorded } \\
\text { on the DigiGait }\end{array}$ & $\downarrow$ & $\pi$ \\
\hline \multicolumn{4}{|l|}{ Gait rhythm } \\
\hline $\begin{array}{l}\text { Fore limb } \\
\text { stance time }\end{array}$ & $\begin{array}{l}\text { time the paw is in contact } \\
\text { with the ground, influences } \\
\text { the gait rhythm }\end{array}$ & - & - \\
\hline
\end{tabular}




\begin{tabular}{|c|c|c|c|}
\hline $\begin{array}{l}\text { Fore limb } \\
\text { swing time }\end{array}$ & $\begin{array}{l}\text { time the paw is in the air, } \\
\text { influences the gait rhythm }\end{array}$ & - & - \\
\hline $\begin{array}{l}\text { Fore limb brake } \\
\text { time }\end{array}$ & $\begin{array}{l}\text { during stance, the time that } \\
\text { the paw area is increasing, } \\
\text { influences the gait rhythm }\end{array}$ & $\uparrow$ & - \\
\hline $\begin{array}{l}\text { Fore limb } \\
\text { propel time }\end{array}$ & $\begin{array}{l}\text { during stance, the time that } \\
\text { the paw area is decreasing, } \\
\text { influences the gait rhythm }\end{array}$ & $\downarrow$ & - \\
\hline $\begin{array}{l}\text { Hind limb } \\
\text { stance time }\end{array}$ & $\begin{array}{l}\text { time the paw is in contact } \\
\text { with the ground, influences } \\
\text { the gait rhythm }\end{array}$ & - & - \\
\hline $\begin{array}{l}\text { Hind limb } \\
\text { swing time }\end{array}$ & $\begin{array}{l}\text { time the paw is in the air, } \\
\text { influences the gait rhythm }\end{array}$ & - & - \\
\hline $\begin{array}{l}\text { Hind limb brake } \\
\text { time }\end{array}$ & $\begin{array}{l}\text { during stance, the time that } \\
\text { the paw area is increasing, } \\
\text { influences the gait rhythm }\end{array}$ & $\lambda$ & $\pi$ \\
\hline $\begin{array}{l}\text { Hind limb } \\
\text { propel time }\end{array}$ & $\begin{array}{l}\text { during stance, the time that } \\
\text { the paw area is decreasing, } \\
\text { influences the gait rhythm }\end{array}$ & - & - \\
\hline \multicolumn{4}{|l|}{ Gait posture } \\
\hline $\begin{array}{l}\text { Absolute paw } \\
\text { angle }\end{array}$ & $\begin{array}{l}\text { angle between the paw and } \\
\text { the snout-tail axis, gait } \\
\text { posture parameter }\end{array}$ & $y$ & $\uparrow$ \\
\hline $\begin{array}{l}\text { Paw angle } \\
\text { variability }\end{array}$ & $\begin{array}{l}\text { standard deviation of the } \\
\text { paw angle, gait posture } \\
\text { parameter }\end{array}$ & $\lambda$ & - \\
\hline Shared stance & $\begin{array}{l}\text { percentage of hind limb } \\
\text { stance time that both hind } \\
\text { limbs are in contact with the } \\
\text { ground at the same time, } \\
\text { gait posture parameter }\end{array}$ & $\uparrow$ & - \\
\hline $\begin{array}{l}\text { Paw placement } \\
\text { positioning }\end{array}$ & $\begin{array}{l}\text { extent of overlap between } \\
\text { ipsilateral fore and hind } \\
\text { paws during full stance, gait } \\
\text { posture parameter }\end{array}$ & $\searrow$ & - \\
\hline
\end{tabular}

In the endophilin mutants, slight changes were observed in body weight and muscle strength. These changes were further enhances by Fbxo32 reduction. The strongest motor impairment in endophilin mutants was the fall latency from the Rotarod. This impairment was partially rescued in endophilin mutants that are HT for Fbxo32. However, the reduction of Fbxo32 had little to no effect on the gait rhythm of both fore 
and hind limbs, but tended to ameliorate the paw angle and positioning in endophilin 1KO-2HT-3KO mice.

In summary, though endophilin mutants with partial genetic deletion of Fbxo32 still display some motor impairments, especially in the grip strength, motor coordination is significantly improved in these mutants. These findings are in accordance with the hypothesis that genetic reduction of $\mathrm{Fbxo} 32$ has a positive effect on the motor disabilities observed in endophilin mutants. While there are still many open questions in this area, the data presented here show that the modulation of a protein involved with proteostasis (through the UPS and autophagy) directly affects the motor behavior of a living mouse model for defective endocytosis and autophagy. 


\section{Discussion}

Defects in endocytosis have previously been linked to neurodegeneration (Ralser, et al., 2005; Ren, et al., 2008; Shi, et al., 2009; Trempe, et al., 2009; Milosevic, et al., 2011; Matta, et al., 2012; Edvardson, et al., 2012; Köroğlu, Baysal, Cetinkaya, Karasoy, \& Tolun, 2013; Ambroso, Hegde, \& Langen, 2014; Arranz, et al., 2015). While the evidence of a connection between the two processes is compelling, a concrete mechanism, which also factors in the aspect of aging, is still missing. This study aims to shed further light into the question if defects in endocytosis per se cause neurodegeneration or if the observed phenotypes from previous studies stem from secondary or completely unrelated processes.

The endophilin-A mouse model offers a great potential to study the onset and progression of neurodegeneration in a model that has various degrees of protein $\mathrm{KO}$ due to its presence in three closely related genes. While endophilin TKO have a very strong phenotype and die within a few hours after birth, endophilin 1 single KOs show no apparent phenotype, with other mutants displaying mild to strong phenotypes depending on the number of missing endophilin alleles and the age.

Several publications about the endophilin mouse model were conducted on endophilin TKO and DKO animals (Milosevic, et al., 2011; Cao, Milosevic, Giovedi, \& De Camilli, 2014), yet little work has been done on aging endophilin 1KO-2HT-3KO mice. This study gives a detailed description of the motor abilities of endophilin $1 \mathrm{KO}-$ $2 \mathrm{HT}-3 \mathrm{KO}$ animals and compares biochemical and immunohistochemistry data from these animals to endophilin TKO and DKO brains. 


\subsection{Motor impairments in endocytic mutants}

Of the many links between endocytic proteins and neurodegeneration, the overarching majority points to a connection to PD (Shi, et al., 2009; Trempe, et al., 2009; Matta, et al., 2012; Ambroso, Hegde, \& Langen, 2014; Arranz, et al., 2015; Soukup, et al., 2016; Cao, et al., 2017; Quadri, et al., 2013; Krebs, et al., 2013; Olgiati, et al., 2014). In PD, dopaminergic neurons are degenerating, causing problems with motor planning and coordination. As a result, many symptoms of PD patients can be recognized by people without medical training as the motor impairments, especially in the gait, are often very prominent. In this thesis, the progression of motor impairments was therefore used as an indicator for neurodegeneration in endocytic mutants.

The most striking observation when testing the motor abilities of various endophilin mutants is the progressive decrease of fall latency from the Rotarod (Figure 24). The test is designed to measure motor coordination and concentration. This decrease in fall latency in the endophilin mouse model depends on the age (as it happens in all groups including the WT control group) and on the number of missing endophilin alleles. While the difference to the WT group is already significant in endophilin $1 \mathrm{KO}$ $2 \mathrm{HT}-3 \mathrm{KO}$ mice at the age of 1 month, for endophilin 1KO-2HT-3WT mice it becomes only significant at 3 months of age and never for endophilin 1KO-2WT-3WT mice.

The finding also suggests a more important role of endophilin 2 than of endophilin 3 in motor coordination: Compared to the endophilin 1 single KO (endophilin 1KO-2WT3WT), endophilin 1KO-2HT-3WT mice carry only an additional $50 \%$ deletion of endophilin 2 expression, yet the motor coordination is severely decreased in the latter group but not in the former. However, endophilin 1KO-2WT-3KO mice, which compared to endophilin 1KO-2WT-3WT mutants carry an additional full deletion of endophilin 3, perform better during the task than endophilin 1KO-2HT-3WT mice. The difference between endophilin 2 and 3 is made even more evident by comparing the life expectancy of endophilin 1,2 DKOs and 1,3 DKOs (endophilin 1KO-2WT-3KO): endophilin 1,2 DKOs die within 3 weeks after birth, while endophilin 1,3 DKOs live for around 18 months. 
The reason for this might be the fact that endophilin 3 accounts for only $3-5 \%$ of all endophilin protein in the brain, while endophilin 2 accounts for $10-15 \%$ and endophilin 1 for $80-85 \%$ (Milosevic, et al., 2011 and unpublished data from Dr. Ira Milosevic). Therefore, the loss of endophilin 3 might be more easily compensated for by the remaining other endophilins. Another explanation might be the different distribution of the three endophilins. While endophilin 1 and 3 are mostly brain specific (plus little expression of endophilin 3 in the testis), endophilin 2 is expressed ubiquitously (Giachino, et al., 1997; Ringstad, Nemoto, \& De Camilli, 1997; So, et al., 2000). Therefore, the loss of endophilin 2 could potentially have negative effects in organs other than the brain that could not be compensated for by endophilin 1 or 3. However, another BAR protein, amphiphysin, is similar to endophilin in structure and binding properties and is expressed ubiquitously. It may be able to compensate to some degree for the loss of endophilin 2 (Wu, Matsui, \& Tomizawa, 2009; Dong, Gou, Li, Liu, \& Bai, 2015; Tsutsui, Maeda, Tsutsui, Seki, \& Tokunaga, 1997; Ren, Vajjhala, Lee, Winsor, \& Munn, 2006).

Before tests were conducted, general health and fitness were always assessed for the endophilin mutants. The body weight and grip strength of endophilin $1 \mathrm{KO}-2 \mathrm{HT}-$ 3KO mice compared to WT mice progressively decreases with aging (Figure 23). This could potentially contribute to the poor performance of these animals on the Rotarod. However, endophilin 1KO-2WT-3WT mice have even lower relative grip strength but still perform as well as WT animals in the Rotarod task.

The poor motor performance is also observed in the maximal running speed on the DigiGait (Figure 25). The ability to run at the highest speed on the treadmill progressively decreases with age in endophilin $1 \mathrm{KO}-2 \mathrm{HT}-3 \mathrm{KO}$ animals, meaning that it is difficult for these animals to keep up with the treadmill even when it is set to the moderate running speed of $20 \mathrm{~cm} / \mathrm{s}$ that was used for analysis of gait parameters. In light of this, the data obtained from endophilin 1KO-2HT-3KO mice capable of running at this speed has to be interpreted with care as the relative low number of animals that were recorded forms a subset of the group that can be expected to perform better than the non-runners that cannot be analyzed. In addition, the low number of effectively recorded animals also complicated the statistical analysis of this group. 
In PD patients, problems with motor planning and coordination lead to symptoms that manifest in the alteration of gait rhythm and posture (Bovonsunthonchai, Vachalathiti, Pisarnpong, Khobhun, \& Hiengkaew, 2014; Ellis, et al., 2015; Grajić, Stanković, Radovanović, \& Kostić, 2015). Video based gait analysis offers the possibility to evaluate about 50 gait parameters that describe all aspects of walking (Rostosky \& Milosevic, 2018). This approach revealed that the timing of the gait phases swing and stance time is overall unchanged in endophilin mutants compared to WT in neither fore nor hind limbs (Figure 26 and Supplemental Figure 1). Still, when dissecting the stance time into brake and propel time, endophilin mutants showed a strong tendency to increase brake time and thereby decrease propel time in their fore limbs. Like the other motor impairments, this change in gait timing becomes more prominent with aging. The exact meaning of this change can only be speculated, but could indicate a delay in the fore limbs before the initiation of the next step.

Interestingly, this phenotype was not observed in the hind limbs of the same animals. Generally speaking, differences between hind and fore limbs are likely because of slightly different functions of the limbs: hind limbs have a much higher muscle mass than fore limbs and can therefore contribute more acceleration. As the fore limbs are in the front of the animals they likely function to determine which direction the animal is going to. It has previously been published that gait dynamics may differ between fore and hind limbs (Amende, et al., 2005). In the hind limbs of endophilin mutants there are no meaningful alterations of gait or stance timing. It is unclear why brake and propel time are changed in fore but not in hind limbs. One possible explanation is that the finer coordination in the fore limbs is impaired, but the more general swing/stance timing is compensated by the strong acceleration of the hind limbs. Because of this, the lack of coordination only becomes visible in the timing of the brake and propel during stance.

The second group of parameters measured by video-based gait analysis concerns the posture of the animals during running (Figure 27). Endophilin mutants have an overall decrease in their paw angle. High paw angles are thought to be a sign for posture instability and have been reported in a model of demyelinating disease (Powell, Anch, Dyche, Bloom, \& Richter, 1999). However, in an acute model for PD, 
mice treated with 1-methyl-4-phenyl-1,2,3,6-tetrahydropyridine (MPTP), the paw angle of hind limbs also trended to be smaller than in WTs (Amende, et al., 2005), showing that both smaller and higher paw angles are observed in different mouse models for neurological diseases.

One parameter that is often measured in patients with gait anomalies is the percentage of shared stance, also known as double support. It is the percentage of the stance time that both feet are on the ground at the same time and is known to be increased in PD patients (Hausdorff, Cudkowicz, Firtion, Wei, \& Goldberger, 1998; Bryant, et al., 2011). This parameter can roughly be translated to the hind limb double support in mice. This was found to be increased in endophilin mutants, particularly with aging. An increase is often interpreted as a sign of instability as the shared stance allows re-balancing in between the steps.

Taken together, the data reported in the first part of this thesis show that endophilin mutants display mild to strong motor impairments depending on the test age and the severity of the endophilin deletion. Given endophilin's well-established role in endocytosis, this data points in the direction suggested by the above-mentioned literature, namely that defective endocytosis may be related to neurodegeneration, or, more specifically, a PD like phenotype. However, it is unclear if the observed phenotype is a direct consequence of defective endocytosis or if it might be caused by potential additional roles of endophilin.

One approach to answer that question is to study motor behavior in other endocytic mutants: if they give similar results as the endophilin model, this would suggest a common cause for a common phenotype. The results obtained in this thesis from aged dynamin $1 \mathrm{HT}-3 \mathrm{KO}$ and synaptojanin $1 \mathrm{HT}$ mice were to some degree contradictory. Synaptojanin-1 deficient mice had no motor impairments in any of the tests used while dynamin mutants have reduced fall latency from the Rotarod though not as low as that of endophilin 1KO-2HT-3KO mice (Figure 28 and Figure 29).

As the motor impairments in the endophilin mutants are only visible when a critical amount of endophilin is deleted (endophilin 1KO-2WT-3WT mice have no phenotype), it is possible that the $50 \%$ genetic reduction of synaptojanin- 1 is not sufficient to cause 
motor deficits. Interestingly, another model of synaptojanin-1 deficiency was published during the course of this thesis. This model carries a R258Q synaptojanin1 mutation that was previously identified in patients of Early-Onset Parkinsonism (Cao, et al., 2017; Krebs, et al., 2013; Quadri, et al., 2013; Olgiati, et al., 2014). Mice carrying a knock-in (KI) for this mutated synaptojanin-1 instead of WT synaptojanin-1 have defects in endocytosis similar to those described in synaptojanin-1 KO mice, yet unlike the full KO animals, the synaptojanin-1 R258Q KI mice survived until adulthood and could thus be used for motor behavior tests.

In fact, the authors show that synaptojanin-1 R258Q KI mice have obvious motor impairments as assessed by grip strength, Rotarod and other tests though their gait is normal (Cao, et al., 2017). In light of this, it is likely that aged synaptojanin-1 HT mice simply have enough synaptojanin-1 left to fulfill normal function while mice with synaptojanin-1 mutation show similar motor deficits as endophilin and dynamin mutants. In summary, deficiency of any of the three endocytic proteins endophilin, synaptojanin-1 and dynamin can cause motor deficits, but they may only become obvious when the deficiency is strong.

\subsection{Neurodegeneration in the endophilin mouse model}

Neurodegeneration is defined as the death of neurons in the CNS. In the various known neurodegenerative diseases, this cell death occurs in different brain regions and therefore affects different brain functions. In order to understand if the motor deficits in endophilin mutants are accompanied by cell death in the CNS, cryosections from the brains of these mutants were analyzed for different markers of cell death and neurodegeneration. This work was primarily done on endophilin DKO mice as these mice have a very strong phenotype that includes cerebellar ataxia and because neurodegeneration in the form of retraction of the climbing fibers in the cerebellum of these animals has already been published (Milosevic, et al., 2011). Endophilin TKO mice have an even stronger phenotype, but as they die shortly after birth, it is more difficult to discriminate between developmental and degenerative deficits. 
TUNEL staining for apoptotic and necrotic cells showed that there is an increase of cell death in several brain regions of the endophilin DKO brains (Figure 30). The extent of this cell death is relatively low and the overall morphology of the brain is not changed (i.e. no shrinking of specific brain parts). A slight increase in cell death was also detected in 18-month-old endophilin 1KO-2HT-3KO mice (Figure 31). However, the same assay in endophilin TKO brain sections showed a strong background TUNEL signal in the WT brains that was about as strong as the signal in endophilin TKO brains. This could be due to normal post-natal developmental apoptosis (Galluzzi, et al., 2009). It is possible that because endophilin TKO brains were collected shortly after birth and TUNEL staining detects only the final stages of apoptosis, additional cell death through the effect of endophilin TKO was not detected with this test.

To test this hypothesis, apoptosis in endophilin TKO brains was further measured by immunohistochemistry for the early apoptosis marker cleaved caspase 3 (Figure 33). Indeed, using this test, an increase in the number of early apoptotic cells was found in endophilin TKO brain sections.

As synaptojanin-1 KO mice have a similar phenotype as endophilin TKOs with regard to defective endocytosis (Milosevic, et al., 2011; Cremona, et al., 1999), cell death in these mutants was also assessed by cleaved caspase 3 staining. While there was a tendency to an increased cell death, the difference was not significant (Figure 34). This suggests that the timing of cell death is different in the distinct endocytic mutants, or that the slight cell death in endophilin TKO brains is caused by a CME-independent mechanism.

While the cell death detected in endophilin DKO was mild, it was accompanied by a relatively strong astrogliosis, visualized by GFAP immunostaining (Figure 32). Astroglia support neurons and their up-regulation could be an attempt to aid the dying cells to avoid apoptosis (Khakh \& Sofroniew, 2015). The strong activation of astroglia might be an indication that they are successfully providing the cells with nutrients and neurotransmitters that they lack, as they can no longer obtain these by endocytosis, and thereby minimize the observed cell death. It is noteworthy, that the strong difference in GFAP signal between endophilin DKO and WT brain slices might be 
further enhanced by the fact that GFAP is expressed in very low levels in healthy conditions that are sometimes below the detection rate (Khakh \& Sofroniew, 2015). This means that the number of astroglia in WT brains could be similar to that of endophilin DKOs; they might simply not express enough GFAP to be detected.

As both the literature and the observation of motor disabilities in endocytic mutants point to a connection between defective endocytosis and PD, $\alpha$-synuclein, a marker for this particular disease, was examined more closely. In fact, a-synuclein levels were reduced in endophilin mutants and larger puncta were detected in endophilin DKO brain section than in control sections (Figure 35). However, when comparing the spine density or the frequency of mature spines, no differences between WT and endophilin DKO brain sections were found (Figure 36).

Taken together, signs of cell death were found in endophilin mutants, but the extend of that cell death is relatively mild. While in the endphilin DKOs, the cell death is accompanied by a gliosis, no direct connections to a specific cell type or region were found with the methods employed here. More detailed experiments using several celltype specific markers are necessary to investigate if a specific cell type is more prone to cell death in the endophilin mutants or if the slight increase in cell death might be caused by defective endocytosis resulting in the death of neurons distributed throughout the brain. It may also be worth it to perform more experiments that focus on neurodegeneration in the cerebellum. As this brain region is involved in motor coordination and has been reported to show signs of neurodegeneration in endophilin DKO mice (Milosevic, et al., 2011), further investigation could possibly shed light onto the motor deficits found in aged endophilin mutants. 


\subsection{Altered autophagy in endocytic mutants}

In addition to endophilin's well established role in endocytosis (Cestra, et al., 1999; Verstreken, et al., 2003; Sundborger \& Hinshaw, 2014), data presented in this thesis and elsewhere (Murdoch, et al., 2016; Soukup, et al., 2016) indicate that endophilin may also be implicated in autophagy. In the endophilin mouse model, the strongest indication for this additional role is the robust up-regulation of the E3 ubiquitin ligase Fbxo32 in different endophilin mutants that was confirmed for aged endophilin $1 \mathrm{KO}$ 2HT-3KO mice in this thesis (Figure 37).

Fbxo32 was originally described as an E3 ubiquitin ligase that is part of an SCF complex (Bodine, et al., 2001; Gomes, Lecker, Jagoe, Navon, \& Goldberg, 2001) but was afterwards found to also play a role in autophagy and was, in fact, suggested to act as a mediator between the UPS system and autophagy (Zaglia, et al., 2014). This is validated by several studies that report both loss and overexpression of Fbxo32 can have potentially negative effects on cells, especially muscles (Bodine, et al., 2001; Zaglia, et al., 2014; Bühler, et al., 2016; Sakai, et al., 2017; Lee, Kim, Kim, Choi, \& Nam, 2017).

In agreement with this hypothesis, there is an increase in the number of proteins that are marked for degradation (Figure 39) and a decrease in the autophagy marker Atg5 (Figure 41) in aged endophilin mutants. These and other markers have been reported to be changed in a similar way in endophilin DKO and TKO brains (Murdoch, et al., 2016). Moreover, it has been shown that Fbxo32 and endophilin co-localize in WT MEFs on autophagic structures and that reduction of endophilin leads to a decrease of Atg5 and LC3 positive structures (Murdoch, et al., 2016; Soukup, et al., 2016).

Impairments in autophagy in the endophilin mouse model could also contribute to the observed motor deficits and mild neurodegeneration. It has previously been suggested that defects in autophagy can cause neurodegeneration (Douglas \& Dillin, 2010; Nixon, 2013; Liang \& Sigrist, 2018). A recent study further points to a connection of defective autophagy to motor dysfunction in aged mice (Sato, et al., 2018). It seems likely that the observed motor deficits in the endophilin mouse model 
are caused by a combination of defective endocytosis and a dysregulation of autophagy.

As endocytosis is a transport processes that regulates the internalization of different proteins important for several cellular processes, it is possible that the failure of autophagy is an indirect effect of defective endocytosis in endocytic mutants. Two autophagy factors, Atg9a and Atg16I do in fact are trafficked through CME as part of their normal trafficking and aid in the formation of the autophagophore (Ravikumar, Moreau, Jahreiss, Puri, \& Rubinsztein, 2010; Puri, Renna, Bento, Moreau, \& Rubinsztein, 2014). Both proteins are present at normal levels in endophilin TKO and DKO brains (Figure 42). While this finding does not exclude the possibility that other autophagy factors might be present in reduced levels because of defective endocytosis and thereby cause the autophagy failure, it seems unlikely that these two proteins, which are known to undergo CME, are not affected by defective endocytosis while others are.

To further assess the connection between endocytosis and proteostasis, brains from dynamin 1,3 DKO and synaptojanin-1 KO mice were tested for poly-ubiquitin and Atg5 levels (Figure 40, Figure 43). These proteins were found to be unchanged, pointing to a more direct role of endophilin in autophagy. However, recent studies in zebrafish and drosophila have shown that synaptojanin-1 likely does play a role in autophagy (George, Hayden, Stanton, \& Brockerhoff, 2016; Vanhauwaert, et al., 2017). Interestingly, the first study was performed in highly active zebrafish cone photoreceptors and found that the synaptojanin-1 5-phosphatase domain is required for functional autophagy and endocytosis (George, Hayden, Stanton, \& Brockerhoff, 2016). The second study focused on the drosophila neuromuscular junction and human derived induced neuronal cell lines and reports that the second phosphatase domain in synaptojanin-1, the SAC-1 domain, is in fact responsible for autophagy but has no role in endocytosis (Vanhauwaert, et al., 2017).

Of note, the mouse study that used the same human synaptojanin-1 mutation as Vanhauwaert et al. and that was previously reported to be found in patients of EarlyOnset Parkinsonism (Krebs, et al., 2013; Quadri, et al., 2013; Olgiati, et al., 2014), 
reported defects in endocytosis in mice carrying this mutation. This study did not investigate autophagy (Cao, et al., 2017).

While these three studies contradict each other to some degree and further work in this field will be needed to understand the mechanisms, it seems likely that the 5phosphatase domain functions in endocytosis, as has been suggested by several studies (McPherson, et al., 1996; Cremona, et al., 1999; Mani, et al., 2007; Changlleto, et al., 2012). The SAC1 domain, on the other hand, probably has a distinct or additional function, and likely is involved in autophagy (Soukup, Vanhauwaert, \& Verstreken, 2018). Again, the existence of two distinct phosphatase domains with possibly distinct functions, together with the contradiction in the literature as to if the SAC1 domain plays a role in endocytosis, makes it difficult to distinguish if the connection between synaptojanin-1 deficiency and impaired autophagy is because of defective endocytosis or because of distinct functions of this protein independent of endocytosis.

This also has some influence on the interpretation of the motor deficits in these animals. The mutation in synaptojanin-1 R258Q KI mice, which reportedly have motor impairments (Cao, et al., 2017), is located in the SAC1 domain. While in the same study the authors described endocytic defects in these mutants, it is unknown whether they also have autophagy impairments as might be expected given the same mutation leads to problems with autophagosome maturation in drosophila (Vanhauwaert, et al., 2017). As long as it remains unclear if synaptojanin-1 R258Q KI mice have autophagy impairments, it is difficult to judge if the motor deficits in synaptojanin-1 R258Q KI mice are caused by defective endocytosis, impaired autophagy or a combination of both.

In summary, both endophilin and synaptojanin-1 deficiency can lead to motor deficits, neurodegeneration and autophagy impairments. It remains to be clarified how the observed phenotypes influence each other and what is the ultimate cause for the motor deficits in both models. 


\subsection{Partial rescue of endophilin phenotype by Fbxo32 reduction}

Another way to dissect the influence of endocytosis and autophagy impairments on motor behavior is to improve autophagy in the endophilin mutants. Given Fbxo32's proposed role as a mediator between the UPS and autophagy and thereby proteostasis, we hypothesized that the elevated levels of Fbxo32 in the endophilin model could cause the imbalance in autophagy. As a result, endophilin mutant mice were crossed with Fbxo32 KO mice and the genetic reduction of Fbxo32 was confirmed by PCR (Figure 46).

Fbxo32 KO mice have a mild reduction in grip strength at a young age but their motor behavior until the age of 9 months is largely normal. Previous reports on Fbxo32 mice suggest that they have a normal phenotype until the age of 9 months but then develop autophagy impairments and cardiomyopathy until the age of 16 months (Zaglia, et al., 2014). As a result, this thesis did not aim to completely delete Fbxo32 from endophilin mutants, but rather to have a genetic reduction of $\mathrm{Fbxo32}$ to $50 \%$ expression levels to counteract the 2 -fold up-regulation of Fbxo32 detected by NGS and qPCR (Murdoch, et al., 2016).

Therefore, the most interesting genotype to understand the consequences of Fbxo32 reduction on motor behavior were Fbxo32 $\mathrm{HT}$-endophilin 1KO-2HT-2KO mice as endophilin 1KO-2HT-3KO mice had the most obvious motor deficits that are visible already at a young age. Interestingly, the reduction of Fbxo32 levels did indeed ameliorate motor performance. This was most obvious on Rotarod performance, which trends to increase in all endophilin mutants with reduced Fbxo32 expression and is statistically significant for Fbxo32 HT-endophilin 1KO-2HT-3KO animals (Figure 48).

Genetic reduction of Fbxo32 expression decreased the grip strength in young endophilin mutants. This phenotype might be an early indicator for the muscle atrophy that occurs in Fbxo32 KO mice at the age of 16 months (Bodine, et al., 2001; Zaglia, et al., 2014). However, the reduction in grip strength does not deteriorate with age and is in fact not present in 9-month-old Fbxo32 HT-endophilin 1KO-2HT-3KO mice (Figure 47). 
The amelioration of motor behavior in endophilin mutants through the genetic reduction of Fbxo32 indicates that the high expression levels of Fbxo32 in the endophilin mouse model could partially cause the observed motor deficits. The motor coordination is only partially rescued which can be explained by two theories:

- The motor impairments in endophilin mutants are only caused by autophagy misregulation. While genetic reduction of Fbxo32 is beneficial, it does not bring the relative Fbxo32 / endophilin levels into perfect balance, as a consequence the motor behavior is not fully rescued.

- The motor impairments in endophilin mutants are caused by deficiencies in endocytosis AND autophagy. Genetic reduction of Fbxo32 helps to improve the autophagy failure and the remaining difference to the WT motor performance is caused by the still defective endocytosis.

The literature published to this date indicates that a tight regulation of Fbxo32 is important to coordinate proteostasis (Zaglia, et al., 2014; Murdoch, et al., 2016). Specifically, fibroblasts that overexpress Fbxo32 die earlier and have increased caspase activity, but this phenotype can be rescued by a co-overexpression of any of the three endophilins (Murdoch, et al., 2016). Therefore, it is realistic that even a small misbalance in this protein can eventually lead to an impaired protein turnover which over time can have tremendous effects on synapses. These structures are especially vulnerable to proteostasis failure, as they are located far away from the protein translation machinery around the nucleus and have to keep up the tightly regulated processes important for synaptic transmission (Douglas \& Dillin, 2010; Nixon, 2013; Liang \& Sigrist, 2018; Soukup, Vanhauwaert, \& Verstreken, 2018).

On the other hand side, it is striking that synaptojanin-1 R258Q KI and dynamin $1 \mathrm{HT}$ 2KO mice display motor deficits similar to those of endophilin mutants, though they are less pronounced (see above and Cao, et al., 2017). This can indicate that defects in endocytosis can cause motor deficits by themselves, or that synaptojanin and dynamin are also related to autophagy. Recently, some publications were able to show a role for synaptojanin in autophagy (George, Hayden, Stanton, \& Brockerhoff, 2016; Vanhauwaert, et al., 2017). 
There is currently no study about autophagy in a dynamin animal model, but some basic studies already showed that dynamin could potentially be involved in autophagy as well (Totta, Busonero, Leone, Marino, \& Acconcia, 2016; Fang, et al., 2016). In light of this, the two processes of endocytosis and autophagy might be more closely related than previously expected. After all, both processes require the organization of membrane to transport cellular components to their destination. Because of this, it is not unrealistic, that some of the involved players, namely endophilin, synaptojanin and possibly dynamin, are the same for both processes. 


\subsection{Endophilin's role in synaptic proteostasis - A model}

Taking into consideration the data reported here and in the literature about endocytic proteins and autophagy, it seems likely that endophilin plays an additional role in autophagy (reviewed in Soukup, Vanhauwaert, \& Verstreken, 2018). Endophilin can sense and induce membrane curvature through its N-BAR domain (Bai, Hu, Dittman, Pym, \& Kaplan, 2010; Farsad, et al., 2001; Gallop, et al., 2006; Masuda, et al., 2006). Interestingly, the binding affinity is regulated through the PD protein LRRK2 (Ambroso, Hegde, \& Langen, 2014; Soukup, et al., 2016).

The role of endophilin in autophagy could therefore be to recruit membrane, possibly from the membrane or from endosomes, for the initiation of the phagophore which is the first step in the autophagy process. This would explain why endophilin is observed on early autophagosomes, but only to a lesser extent on late autophagosomes (Murdoch, et al., 2016). In this model, the regulation of the Pls during the transport

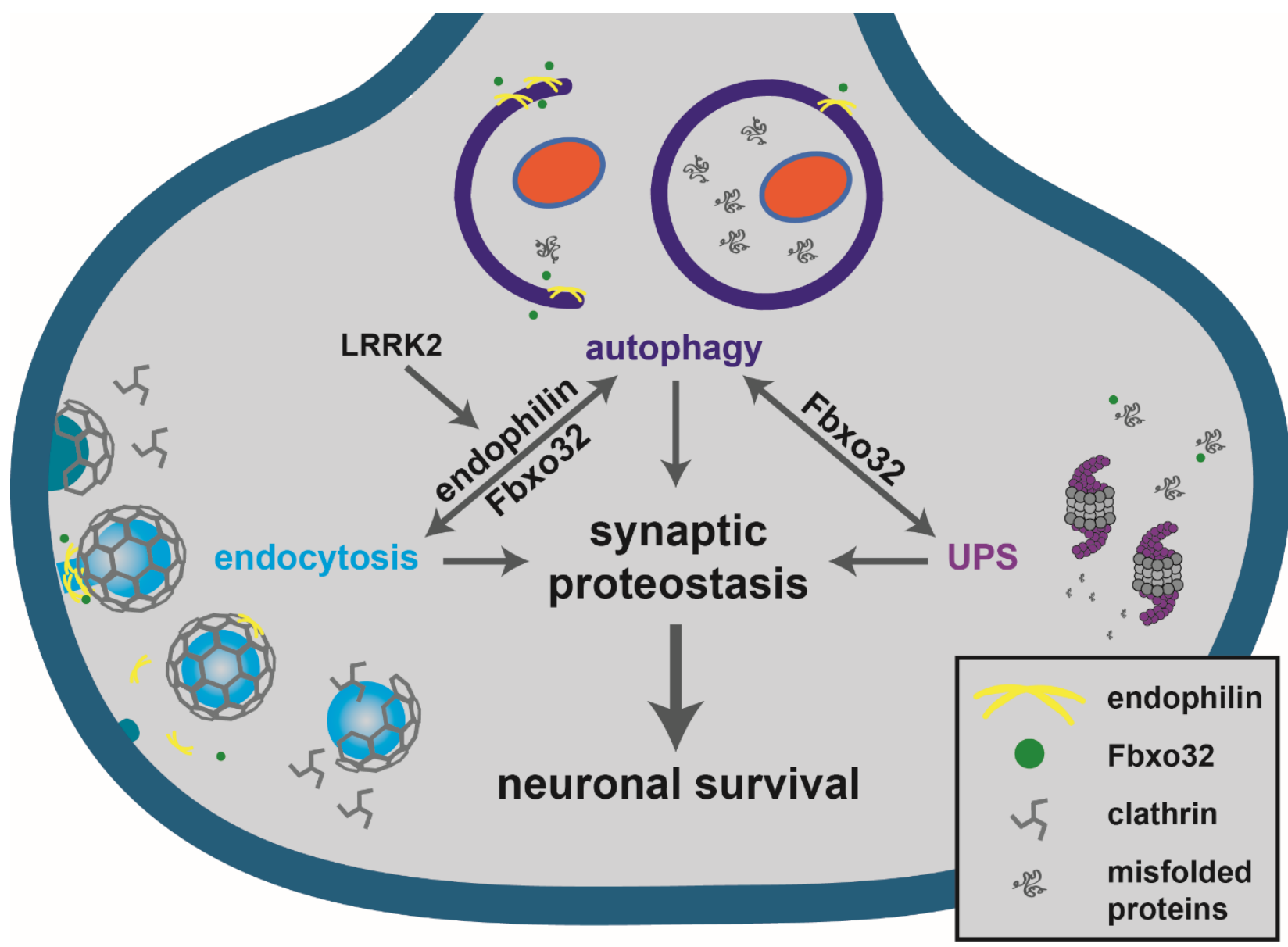


and on the forming autophagosome might be regulated by synaptojanin-1 which is recruited through endophilin (Milosevic, et al., 2011; Cestra, et al., 1999; Verstreken, et al., 2003).

Fbxo32 has been reported to co-localize with endophilin on autophagic (Murdoch, et al., 2016) and endocytic structures (Figure 44). It was also found to be able to tabulate membranes (Murdoch, et al., 2016). It remains unclear if Fbxo32 has a direct role in autophagy or acts mostly as a regulator through its action as an E3 ubiquitin ligase. More work will be needed to understand the specific mechanisms of how endocytic proteins and Fbxo32 work on autophagy, but it seems evident at this point that proteins involved in endocytosis and autophagy are critical for proteostasis at the synapse and therefore, in the long run, for neuronal survival. 


\section{Summary and Conclusion}

Ever since the discovery of neurodegenerative diseases, scientists have wondered about the cause of these detrimental diseases. The large number of studies published on this topic has taught us that there is no straightforward answer to this question; rather that genetic and environmental influences as well as aging contribute to the disruption of different proteins and cellular processes that ultimately cause cell death in the affected brain regions. Recently, an interesting hypothesis has emerged suggesting that protein dysregulation in various neurodegenerative diseases eventually disrupts synaptic proteostasis as a common denominator and thereby causes - or at least contributes to - neurodegeneration.

Synaptic proteostasis comprises protein synthesis, trafficking, endocytosis and protein degradation by the UPS and/or autophagy. Endophilin-A has been well studied for its role in endocytosis but data presented here and elsewhere imply that endophilin-A has an additional, previously unknown role in autophagy. This double function makes endophilin an interesting protein for investigating synaptic proteostasis. As endophilin-A is encoded by three genes in mammals, the complex mouse model brings the advantage of studying the impact of endophilin-A deficiency dependent on the number of deleted endophilin alleles. In fact, using different endophilin-A mutants, data presented in this thesis could show that endophilin deficient mice display motor impairments that deteriorate with aging and that the model displays a mild form of neurodegeneration throughout the brain.

In an effort to discriminate between the impact of disrupted endocytosis and autophagy on the systemic level, the motor abilities of two additional models for defective endocytosis, synaptojanin-1 and dynamin-1,3 deficient mice, were also assessed. No motor deficits were detected in aged synaptojanin-1 HT mice, while some motor impairments were found in dynamin mutants. However, another synaptojanin-1 mouse model was recently published that reportedly displays motor deficits. Instead of a deletion of synaptojanin-1, this model carries a mutation of synaptojanin-1 that was previously identified in patients of Early-Onset Parkinsonism. Interestingly, another study investigated the same mutation in a drosophila model and 
found that autophagy is also disrupted by this mutation. As a result, it remains difficult to judge if defects in endocytosis alone can cause the observed motor deficits.

A second approach to solve this question was the genetic reduction of Fbxo32 in the endophilin-A mouse model. The E3 ubiquitin ligase Fbxo32 is highly up-regulated in endophilin-A mutant brains and was proposed to act as a mediator between the UPS system and autophagy. Hypothetically, reducing Fbxo32 to WT levels could rescue the disruption of autophagy in the endophilin-A deficient mice and consequently the impact of defective autophagy on the systemic level. Indeed, genetic reduction of Fbxo32 ameliorated the motor deficits of endophilin-A mutants. The fact that the motor behavior is ameliorated but not completely rescued could indicate that both defective endocytosis and autophagy impairments contribute to the observed motor deficits in endophilin mutants.

In context with other literature published on the subject, it seems evident that disruption of synaptic proteostasis is potentially harmful to cells, especially to the vulnerable synaptic structures that need to perform critical processes far away from the nucleus. The research of medical strategies that aim to facilitate proteostasis in the brain therefore seems like a promising line of research. Manipulation of autophagy mediators such as Fbxo32 may eventually proof to become an interesting therapeutic target for patients of several neurodegenerative diseases. 


\subsection{Perspectives}

This thesis gives some information about the role of endocytosis and autophagy in neurodegeneration, yet there are still open questions. Endophilin seems to play a role in autophagy, however, the exact function is yet unknown. It can be speculated that endophilin acts through the N-BAR domain to recruit membrane to form the phagophore, but if that membrane is derived from the plasma membrane following endocytosis or if endophilin retrieves it from other organelles is completely unknown at this point. Further studies will be necessary to elucidate the timing, regulation and binding partners of endophilin in this process.

In addition, it is very striking that synaptojanin-1, another endocytic protein and binding partner of endophilin, has recently been suggested to also function in synaptic autophagy. It will be very interesting to learn if the mouse model that carries a synaptojanin-1 mutation discovered in PD patients and was reported to show motor impairments also has autophagy impairments. This has been shown in a drosophila model but so far not in the mouse model.

Besides that, it would also be worthwhile to investigate in more detail if other mouse models for endocytic proteins such as the auxilin mouse model or the dynamin model used here have similar autophagy impairments.

From the data known today, it seems that endocytosis and autophagy are two related processes that are connected by the use of some identical proteins and their function to regulate proteostasis in synapses. 


\section{Bibliography}

Ahle, S., \& Ungewickell, E. (1990). Auxilin, a newly identified clathrin-associated protein in coated vesicles from bovine brain. J Cell Biol., 111(1), pp. 19-29.

Alger, B. E., \& Teyler, T. J. (1976). Long-term and short-term plasticity in the CA1, CA3, and dentate regions of the rat hippocampal slice. Brain Res., 110(3), pp. 463-480.

Ambroso, M. R., Hegde, B. G., \& Langen, R. (2014). Endophilin A1 induces different membrane shapes using a conformational switch that is regulated by phosphorylation. Proc Natl Acad Sci USA, 111(19), 6982-6987.

Amende, I., Kale, A., McCue, S., Glazier, S., Morgan, J. P., \& Hampton, T. G. (2005). Gait dynamics in mouse models of Parkinson's disease and Huntington's disease. J NeuroEngin Rehab, 2(20).

Amstutz, B., Gastaldelli, M., Kälin, S., Imelli, N., Boucke, K., Wandeler, E., . . Greber, U. F. (2008). Subversion of CtBP1-controlled macropinocytosis by human adenovirus serotype 3. EMBO J, 27(7), pp. 956-969.

Andersson, F., Jakobsson, J., Löw, P., Shupliakov, O., \& Brodin, L. (2008). Perturbation of syndapin/PACSIN impairs synaptic vesicle recycling evoked by intense stimulation. $J$ Neuroscience, 28(15), pp. 3925-3933.

Arkhipov, A., Yin, Y., \& Schulten, K. (2009). Membrane-Bending Mechanism of Amphiphysin N-BAR Domains. Biophys J., 97(10), 2727-2735.

Arranz, A. M., Delbroek, L., Van Kolen, K., Guimarães, M. R., Mandemakers, W., Daneels, G., . . Moechars, D. (2015). LRRK2 functions in synaptic vesicle endocytosis through a kinase-dependent mechanism. J Cell Sci., 128(3), pp. 541-552.

Axe, E. L., Walker, S. A., Manifava, M., Chandra, P., Roderick, H. L., Habermann, A., Ktistakis, N. T. (2008). Autophagosome formation from membrane compartments enriched in phosphatidylinositol 3-phosphate and dynamically connected to the endoplasmic reticulum. J Cell Biol., 182(4), pp. 685-701.

Baba, M., Takeshige, K., Baba, N., \& Ohsumi, Y. (1994). Ultrastructural analysis of the autophagic process in yeast: detection of autophagosomes and their characterization. J Cell Biol, 124(6), pp. 903-913.

Backer, J. M. (2008). The regulation and function of Class III PI3Ks: novel roles for Vps34. Biochem J., 410(1), pp. 1-17.

Bai, C., Sen, P., Hofmann, K., Ma, L., Goebl, M., Harper, J. W., \& Elledge, S. J. (1996). SKP1 connects cell cycle regulators to the ubiquitin proteolysis machinery through a novel motif, the F-box. Cell, 86(2), pp. 263-274.

Bai, J., Hu, Z., Dittman, J. S., Pym, E. C., \& Kaplan, J. M. (2010). Endophilin functions as a membrane-bending molecule and is delivered to endocytic zones by exocytosis. Cell, 143(3), 430-441.

Bayer, T. A. (2015). Proteinopathies, a core concept for understanding and ultimately treating degenerative disorders? Eur Neuropsychopharmacol., 25(5), 713-724.

Ben-Saadon, R., Zaaroor, D., Ziv, T., \& Ciechanover, A. (2006). The polycomb protein Ring1B generates self atypical mixed ubiquitin chains required for its in vitro histone H2A ligase activity. Mol Cell, 24(5), pp. 701-711. 
Blood, P. D., \& Voth, G. A. (2006). Direct observation of Bin/amphiphysin/Rvs (BAR) domain-induced membrane curvature by means of molecular dynamics simulations. PNAS, 103(41), 15068-15072.

Blood, P. D., Swenson, R. D., \& Voth, G. A. (2008). Factors Influencing Local Membrane Curvature Induction by N-BAR Domains as Revealed by Molecular Dynamics Simulations. Biophys J., 95(4), 1866-1876.

Bodine, S. C., \& Baehr, L. M. (2014). Skeletal muscle atrophy and the E3 ubiquitin ligases MuRF1 and MAFbx/atrogin-1. Am J Physiol Endocrinol Metab(307), pp. E469-E484.

Bodine, S. C., Latres, E., Baumhueter, S., Lai, V. K., Nunez, L., Clarke, B. A., . . . Glass, D. J. (2001). Identification of ubiquitin ligases required for skeletal muscle atrophy. Science, 294(5547), pp. 1704-1708.

Bonazzi, M., Spanò, S., Turacchio, G., Cericola, C., Valente, C., Colanzi, A., . . Luini, A. (2005). CtBP3/BARS drives membrane fission in dynamin-independent transport pathways. Nat Cell Biol., 7(6), pp. 570-580.

Boucrot, E., Ferreira, A. P., Almeida-Souza, L., Debard, S., Vallis, Y., Howard, G., . . . McMahon, H. T. (2015). Endophilin marks and controls a clathrin-independent endocytic pathway. Nature, 517(7535), pp. 460-465.

Bovonsunthonchai, S., Vachalathiti, R., Pisarnpong, A., Khobhun, F., \& Hiengkaew, V. (2014). Spatiotemporal gait parameters for patients with Parkinson's disease compared with normal individuals. Physiother Res Int., 19(3), pp. 158165.

Bryant, M. S., Rintala, D. H., Hou, J. G., Charness, A. L., Fernandez, A. L., Collins, R. L., ... Protas, E. J. (2011). Gait variability in Parkinson's disease: influence of walking speed and dopaminergic treatment. Neurol Res., 33(9), pp. 959964.

Bucciantini, M., Giannoni, E., Chiti, F., Baroni, F., Formigli, L., Zurdo, J., . . Stefani, M. (2002). Inherent toxicity of aggregates implies a common mechanism for protein misfolding diseases. Nature, 416(6880), pp. 507-511.

Bühler, A., Kustermann, M., Bummer, T., Rottbauer, W., Sandri, M., \& Just, S. (2016). Atrogin-1 Deficiency Leads to Myopathy and Heart Failure in Zebrafish. Int $J$ $\mathrm{Mol}$ Sci, 17(2), p. e187.

Burke, R. E., \& O'Malley, K. (2013). Axon degeneration in Parkinson's disease. Exp Neurol.(246), pp. 72-83.

Burroughs, A. M., Jaffee, M., lyer, L. M., \& Aravind, L. (2008). Anatomy of the E2 ligase fold: implications for enzymology and evolution of ubiquitin/Ub-like protein conjugation. J Struct Biol., 162(2), pp. 205-218.

Cao, H., Garcia, F., \& McNiven, M. A. (1998). Differential Distribution of Dynamin Isoforms in Mammalian Cells. Mol Biol Cell, 9(9), pp. 2595-2609.

Cao, M., Milosevic, I., Giovedi, S., \& De Camilli, P. (2014). Upregulation of Parkin in endophilin mutant mice. J Neurosci., 34(49), pp. 16544-16549.

Cao, M., Wu, Y., Ashrafi, G., McCartney, A. J., Wheeler, H., Bushong, E. A., . . . De Camilli, P. (2017). Parkinson Sac Domain Mutation in Synaptojanin 1 Impairs Clathrin Uncoating at Synapses and Triggers Dystrophic Changes in Dopaminergic Axons. Neuron, 93(4), pp. 882-896. 
Cenciarelli, C., Chiaur, D. S., Guardavaccaro, D., Parks, W., Vidal, M., \& Pagano, M. (1999). Identification of a family of human F-box proteins. Curr Biol., 9(20), pp. 1177-1179.

Cescatti, M., Saverioni, D., Capellari, S., Tagliavini, F., Kitamoto, T., Ironside, J., . . . Parchi, P. (2016). Analysis of Conformational Stability of Abnormal Prion Protein Aggregates across the Spectrum of Creutzfeldt-Jakob Disease Prions. $J$ Virology, 90(14), pp. 6244-6254.

Cestra, G., Castagnoli, L., Dente, L., Minenkova, O., Petrelli, A., Migone, N., . . . Cesareni, G. (1999). The SH3 domains of endophilin and amphiphysin bind to the proline-rich region of synaptojanin 1 at distinct sites that display an unconventional binding specificity. J Biol Chem., 274(45), pp. 32001-32007.

Chang, Y. Y., \& Neufeld, T. P. (2009). An Atg1/Atg13 complex with multiple roles in TOR-mediated autophagy regulation. Mol Biol Cell, 20(7), pp. 2004-2014.

Chang-lleto, B., Frere, S. G., Chan, R. B., Voronov, S. V., Roux, A., \& Di Paolo, G. (2012). Synaptojanin 1-mediated $\mathrm{PI}(4,5) \mathrm{P} 2$ hydrolysis is modulated by membrane curvature and facilitates membrane fission. Dev Cell, 20(2), pp. 206-218.

Chen, Z. J. (2005). Ubiquitin signalling in the NF-kappaB pathway. Nat Cell Biol., 7(8), pp. 758-765.

Chowdhury, M., \& Enenkel, C. (2015). Intracellular Dynamics of the UbiquitinProteasome-System. F1000Res., 4, p. e367.

Cicchini, M., Karantza, V., \& Xia, B. (2015). Molecular Pathways: Autophagy in Cancer-A Matter of Timing and Context. Clin Cancer Res., 21(3), pp. 498504.

Clayton, E. L., \& Cousin, M. A. (2009). The Molecular Physiology of ActivityDependent Bulk Endocytosis of Synaptic Vesicles. J Neurochem., 111(4), pp. 901-914.

Coux, O., Tanaka, K., \& Goldberg, A. L. (1996). Structure and functions of the $20 \mathrm{~S}$ and 26S proteasomes. Annu Rev Biochem., 65, pp. 801-847.

Cremona, O., Di Paolo, G., Wenk, M. R., Lüthi, A., Kim, W. T., Takei, K., . . . De Camilli, P. (1999). Essential role of phosphoinositide metabolism in synaptic vesicle recycling. Cell, 99(2), pp. 179-188.

Csibi, A., Cornille, K., Leibovitch, M. P., Poupon, A., Tintignac, L. A., Sanchez, A. M., \& Leibovitch, S. A. (2010). The translation regulatory subunit elF3f controls the kinase-dependent mTOR signaling required for muscle differentiation and hypertrophy in mouse. PLoS One, 5(2), p. e8994.

Csibi, A., Tintignac, L. A., Leibovitch, M. P., \& Leibovitch, S. A. (2008). elF3-f function in skeletal muscles: to stand at the crossroads of atrophy and hypertrophy. Cell Cycle, 7(12), pp. 1698-1701.

Cuervo, A. M., Bergamini, E., Brunk, U. T., Dröge, W., Ffrecnch, M., \& Terman, A. (2005). Autophagy and aging: the importance of maintaining "clean" cells. Autophagy, 1(3), pp. 131-140.

Davies, S. W., Turmaine, M., Cozens, B. A., DiFiglia, M., Sharp, A. H., Ross, C. A., . . . Bates, G. P. (1997). Formation of neuronal intranuclear inclusions underlies the neurological dysfunction in mice transgenic for the HD mutation. Cell, 90(3), pp. 537-548. 
Davis, R. L., Weintraub, H., \& Lassar, A. B. (1987). Expression of a single transfected cDNA converts fibroblasts to myoblasts. Cell, 51(6), pp. 987-1000.

Dawson, T. M., \& Dawson, V. L. (2010). The role of parkin in familial and sporadic Parkinson's disease. Mov Disord., 25, pp. 32-39.

Di Fonzo, A., Tassorelli, C., De Mari, M., Chien, H. F., Ferreira, J., Rohé, C. F., . . . Italian Parkinson's Genetics Network. (2006). Comprehensive analysis of the LRRK2 gene in sixty families with Parkinson's disease. Eur J Hum Genet., 14(3), pp. 322-331.

Dice, J. F. (1995). Selective Degradation of Cytosolic Proteins by Direct Transport Into Lysosomes. Memb Prot Transp., 1, pp. 59-75.

Dong, Y., Gou, Y., Li, Y., Liu, Y., \& Bai, J. (2015). Synaptojanin cooperates in vivo with endophilin through an unexpected mechanism. eLife, 4, p. e05660.

Douglas, P. M., \& Dillin, A. (2010). Protein homeostasis and aging in neurodegeneration. Journal of Cell Biology, 190(5), pp. 719-729.

Doyle, D. A., Lee, A., Lewis, J., Kim, E. S., \& MacKinnon, R. (1996). Crystal structures of a complexed and peptide-free membrane protein-binding domain: molecular basis of peptide recognition by PDZ. Cell, 85(7), pp. 1067-1076.

Edmondson, D. G., \& Olson, E. N. (1993). Helix-loop-helix proteins as regulators of muscle-specific transcription. J Biol Chem., 268(2), pp. 755-758.

Edvardson, S., Cinnamon, Y., Ta-Shma, A., Shaag, A., Yim, Y. I., Zenvirt, S., . . . Elpeleg, O. (2012). A deleterious mutation in DNAJC6 encoding the neuronalspecific clathrin-uncoating co-chaperone auxilin, is associated with juvenile parkinsonism. PLoS One, 7(5), p. e36458.

Eisenberg, E., \& Greene, L. E. (2007). Multiple roles of auxilin and hsc70 in clathrinmediated endocytosis. Traffic, 8(6), pp. 640-646.

Ellis, R. J., Ng, Y. S., Zhu, S., Tan, D. M., Anderson, B., Schlaug, G., \& Wang, Y. (2015). A Validated Smartphone-Based Assessment of Gait and Gait Variability in Parkinson's Disease. PLoS One, 10(10), p. e0141694.

Engert, F., \& Bonhoeffer, T. (1999). Dendritic spine changes associated with hippocampal long-term synaptic plasticity. Nature, 399(6731), pp. 66-70.

Eskelinen, E. L. (2005). Maturation of autophagic vacuoles in Mammalian cells. Autophagy, 1(1), pp. 1-10.

Evilsizor, M. N., Ray-Jones, H. F., Lifshitz, J., \& Ziebell, J. (2015). Primer for immunohistochemistry on cryosectioned rat brain tissue: example staining for microglia and neurons. J Vis Exp., 99, p. e52293.

Fader, C. M., \& Colombo, M. I. (2009). Autophagy and multivesicular bodies: two closely related partners. Cell Death Differ., 16(1), pp. 70-78.

Fang, X., Zhou, J., Liu, W. D., Gala, U., Sandoval, H., Jaiswal, M., \& Tong, C. (2016). Dynamin Regulates Autophagy by Modulating Lysosomal Function. J Genet Genomics, 43(2), pp. 77-86.

Farnández-Alfonso, T., Kwan, R., \& Ryan, T. A. (2006). Synaptic vesicles interchange their membrane proteins with a large surface reservoir during recycling. Neuron, 51(2), pp. 179-186.

Farsad, K., Ringstad, N., Takei, K., Floyd, S. R., Rose, K., \& De Camilli, P. (2001). Generation of high curvature membranes mediated by direct endophilin bilayer interactions. J Cell Biol., 155(2), pp. 193-200. 
Ferguson, S. M., Brasnjo, G., Hayashi, M., Wölfel, M., Collesi, C., Giovedi, S., .. . De Camili, P. (2007). A selective activity-dependent requirement for dynamin 1 in synaptic vesicle endocytosis. Science, 316(5824), pp. 570-574.

Ferguson, S. M., Raimondi, A., Paradise, S. S., Mesaki, K., Ferguson, A., Destaing, O., ... De Camilli, P. (2009). Coordinated actions of actin and BAR proteins upstream of dynamin at endocytic clathrin-coated pits. Dev Cell, 17(6), pp. 811822.

Fernández-Alfonso, T., \& Ryan, T. A. (2004). The kinetics of synaptic vesicle pool depletion at CNS synaptic terminals. Neuron, 41(6), pp. 943-953.

Foletta, V. C., White, L. J., Larsen, A. E., Léger, B., \& Russell, A. P. (2011). The role and regulation of MAFbx/atrogin-1 and MuRF1 in skeletal muscle atrophy. Pflugers Arch., 461(3), pp. 325-335.

Fortin, D. A., Monika, A. D., Srivastava, T., Brady, J. D., Nygaard, S., Derkach, V. A., \& Soderling, T. R. (2010). LTP-dependent Spine Enlargement Requires Synaptic Ca2+-permeable AMPARs Recruited by CaM-kinase I. J Neuroscience, 30(35), pp. 11565-11575.

Fuertes, G., Martín De Llano, J. J., Villarroya, A. R., \& Knecht, E. (2003). Changes in the proteolytic activities of proteasomes and lysosomes in human fibroblasts produced by serum withdrawal, amino-acid deprivation and confluent conditions. Biochem J, 375(1), pp. 75-86.

Fujita, N., Itoh, T., Omori, H., Fukuda, M., Noda, T., \& Yoshimori, T. (2008). The Atg16L complex specifies the site of LC3 lipidation for membrane biogenesis in autophagy. Mol Biol Cell, 19(5), pp. 2092-2100.

Fukazawa, Y., Saitoh, Y., Ozawa, F., Ohta, Y., Mizuno, K., \& Inokuchi, K. (2003). Hippocampal LTP is accompanied by enhanced F-actin content within the dendritic spine that is essential for late LTP maintenance in vivo. Neuron, 38(3), pp. 447-460.

Gad, H., Löw, P., Zotova, E., Brodin, L., \& Shupliakov, O. (1998). Dissociation between $\mathrm{Ca} 2+$-triggered synaptic vesicle exocytosis and clathrin-mediated endocytosis at a central synapse. Neuron, 21(3), pp. 607-616.

Gage, G. J., Kipke, D. R., \& Shain, W. (2012). Whole Animal Perfusion Fixation for Rodents. J Vis Exp, 65, p. e3564.

Gallop, J. L., Jao, C. C., Kent, H. M., Butler, P. J., Evans, P. R., Langen, R., \& McMahon, H. T. (2006). Mechanism of endophilin N-BAR domain-mediated membrane curvature. EMBO J., 25(12), pp. 2898-2910.

Galluzzi, L., Aaronson, S. A., Abrams, J., Alnemri, E. S., Andrews, D. W., Baehrecke, E. H., . . Kroemer, G. (2009). Guidelines for the use and interpretation of assays for monitoring cell death in higher eukaryotes. Cell Death Differ, 16(8), pp. 1093-1107.

George, A. A., Hayden, S., Stanton, G. R., \& Brockerhoff, S. E. (2016). Arf6 and the 5 'phosphatase of Synaptojanin 1 regulate autophagy in cone photoreceptors. Inside Cell, 1(2), pp. 117-133.

Giachino, C., Lantelme, E., Lanzetti, L., Saccone, S., Della Valle, G., \& Migone, N. (1997). A Novel SH3-Containing Human Gene Family Preferentially Expressed in the Central Nervous System. Genomics, 41(3), 427-434. 
Gilks, W. P., Abou-Sleiman, P. M., Gandhi, S., Jain, S., Singleton, A., Lees, A. J., . . . Wood, N. W. (2005). A common LRRK2 mutation in idiopathic Parkinson's disease. Lancet, 365(9457), pp. 415-416.

Giráldez-Pérez, R., Antolín-Vallespín, M., Muñoz, M., \& Sánchez-Capelo, A. (2014). Models of $\alpha$-synuclein aggregation in Parkinson's disease. Acta Neuropathol Commun., 2(176).

Glick, D., Barth, S., \& Macleod, K. F. (2010). Autophagy: cellular and molecular mechanisms. J Pathol, 221(1), pp. 3-12.

Gomes, M. D., Lecker, S. H., Jagoe, R. T., Navon, A., \& Goldberg, A. L. (2001). Atrogin-1, a muscle-specific F-box protein highly expressed during muscle atrophy. PNAS, 98(25), pp. 14440-14445.

Grabs, D., Slepnev, V. I., Songyang, Z., David, C., Lynch, M., Cantley, L. C., \& De Camilli, P. (1997). The SH3 domain of amphiphysin binds the proline-rich domain of dynamin at a single site that defines a new $\mathrm{SH} 3$ binding consensus sequence. J Biol Chem, 272(20), pp. 13419-13425.

Grajić, M., Stanković, I., Radovanović, S., \& Kostić, V. (2015). Gait in drug naïve patients with de novo Parkinson's disease--altered but symmetric. Neurol Res., 37(8), pp. 712-716.

Groll, M., Ditzel, L., Löwe, J., Stock, D., Bochtler, M., Bartunik, H. D., \& Huber, R. (1997). Structure of $20 \mathrm{~S}$ proteasome from yeast at 2.4 A resolution. Nature, 386(6624), pp. 463-471.

Guo, S., Stolz, L. E., Lemrow, S. M., \& York, J. D. (1999). SAC1-like domains of yeast SAC1, INP52, and INP53 and of human synaptojanin encode polyphosphoinositide phosphatases. J Biol. Chem., 274(19), pp. 12990-12995.

Hara, T., \& Mizushima, N. (2009). Role of ULK-FIP200 complex in mammalian autophagy: FIP200, a counterpart of yeast Atg17? Autophagy, 5(1), pp. 85-87.

Harris, K. M., \& Stevens, J. K. (1988). Dendritic spines of rat cerebellar Purkinje cells: serial electron microscopy with reference to their biophysical characteristics. $J$ Neuroscience, 8(12), pp. 4455-4469.

Harris, K. M., \& Stevens, J. K. (1989). Dendritic spines of CA 1 pyramidal cells in the rat hippocampus: serial electron microscopy with reference to their biophysical characteristics. J Neuroscience, 9(8), pp. 2982-2997.

Hausdorff, J. M., Cudkowicz, M. E., Firtion, R., Wei, J. Y., \& Goldberger, A. L. (1998). Gait variability and basal ganglia disorders: Stride-to-stride variations of gait cycle timing in parkinson's disease and Huntington's disease. Movement Disorders, 13(3), pp. 428-437.

Hayashi-Nishino, M., Fujita, N., Noda, T., Yamaguchi, A., Yoshimori, T., \& Yamamoto, A. (2009). A subdomain of the endoplasmic reticulum forms a cradle for autophagosome formation. Nat Cell Biol., 11(12), pp. 1433-1437.

Hershko, A., \& Ciechanover, A. (1998). The ubiquitin system. Annu Rev Biochem., 67, pp. 425-479.

Hinshaw, J. E. (2000). Dynamin and its Role in Membrane Fission. Annu. Rev. Cell Dev. Biol., 16(1), pp. 483-519.

Hosokawa, N., Hara, T., Kaizuka, T., Kishi, C., Takamura, A., Miura, Y., . . . Mizushima, N. (2009). Nutrient-dependent mTORC1 association with the ULK1-Atg13-FIP200 complex required for autophagy. Mol Biol Cell, 20(7), pp. 1981-1991. 
Hotulainen, P., \& Hoogenraad, C. C. (2010). Actin in dendritic spines: connecting dynamics to function. J Cell Biol., 189(4), pp. 619-629.

Hotulainen, P., Llano, O., Smirnov, S., Tanhuanpää, K., Faix, J., Rivera, C., \& Lappalainen, P. (2009). Defining mechanisms of actin polymerization and depolymerization during dendritic spine morphogenesis. J Cell Biol., 185(2), pp. 323-339.

Husnjak, K., Elsasser, S., Zhang, N., Chen, X., Randles, L., Shi, Y., . . Dikic, I. (2008). Proteasome subunit Rpn13 is a novel ubiquitin receptor. Nature, 453(7194), pp. 481-488.

Huttner, W. B., \& Schmidt, A. (2000). Lipids, lipid modification and lipid-protein interaction in membrane budding and fission--insights from the roles of endophilin A1 and synaptophysin in synaptic vesicle endocytosis. Curr Opin Neurobiol, 10(5), pp. 543-551.

Jaber, N., \& Zong, W. X. (2013). Class III PI3K Vps34: essential roles in autophagy, endocytosis, and heart and liver function. Ann N Acad Sci., 1280, pp. 48-51.

Jao, C. C., Hegde, B. G., Gallop, J. L., Hegde, P. B., McMahon, H. T., Haworth, I. S., \& Langen, R. (2010). Roles of amphipathic helices and the bin/amphiphysin/rvs (BAR) domain of endophilin in membrane curvature generation. J Biol Chem., 285(26), 20164-20170.

Jogo, M., Shiraishi, S., \& Tamura, T. A. (2009). Identification of MAFbx as a myogenin-engaged F-box protein in SCF ubiquitin ligase. FEBS Letter, 583(17), pp. 2715-2719.

Julie, L.-C., Sabrina, B.-P., Marie-Pierre, L., \& Leibovitch, S. A. (2012). Identification of essential sequences for cellular localization in the muscle-specific ubiquitin E3 ligase MAFbx/Atrogin 1. FEBS Letters, 568, pp. 362-367.

Jung, C. H., Jun, C. B., Ro, S. H., Kim, Y. M., Otto, N. M., Cao, J., . . Kim, D. H. (2009). ULK-Atg13-FIP200 complexes mediate mTOR signaling to the autophagy machinery. Mol Biol Cell, 20(7), pp. 1992-2003.

Kaeser, P. S., Deng, L., Wang, Y., Dulubova, I., Liu, X., Rizo, J., \& Südhöf, T. C. (2011). RIM proteins tether $\mathrm{Ca} 2+$ channels to presynaptic active zones via a direct PDZ-domain interaction. Cell, 144(2), 282-295.

Keren, A., Tamir, Y., \& Bengal, E. (2006). The p38 MAPK signaling pathway: a major regulator of skeletal muscle development. Mol Cell Endocrinol., 252(1-2), pp. 224-230.

Khakh, B. S., \& Sofroniew, M. V. (2015). Diversity of astrocyte functions and phenotypes in neural circuits. Nature Neuroscience, 18(7), pp. 942-952.

Kjaerulff, O., Brodin, L., \& Jung, A. (2011). The structure and function of endophilin proteins. Cell Biochem Biophys., 60(3), 137-154.

Klaips, C. L., Jayaraj, G. G., \& Hartl, F. U. (2017). Pathways of cellular proteostasis in aging and disease. J Cell Biol, 217(1), pp. 51-63.

Klingauf, J., Kavalali, E. T., \& Tsien, R. W. (1998). Kinetics and regulation of fast endocytosis at hippocampal synapses. Nature, 394(6693), pp. 581-585.

Klionsky, D. J. (2007). Autophagy: from phenomenology to molecular understanding in less than a decade. Nat Rev Mol Cell Biol., 8(11), pp. 931-937.

Koga, H., Kaushik, S., \& Cuervo, A. M. (2011). Protein homeostasis and aging: The importance of exquisite quality control. Ageing Res Rev., 10(2), pp. 205-215. 
Koike, M., Shibata, M., Waguri, S., Yoshimura, K., Tanida, I., Kominami, E., . . . Uchiyama, Y. (2005). Participation of autophagy in storage of lysosomes in neurons from mouse models of neuronal ceroid-lipofuscinoses (Batten disease). Am J Pathol., 167(6), pp. 1713-1728.

Köroğlu, Ç., Baysal, L., Cetinkaya, M., Karasoy, H., \& Tolun, A. (2013). DNAJC6 is responsible for juvenile parkinsonism with phenotypic variability. Parkinsonism Relat Disord., 19(3), pp. 320-324.

Krebs, C. E., Karkheiran, S., Powell, J. C., Cao, M., Makarov, V., Darvish, H., . . . Paisán-Ruiz, C. (2013). The Sac1 domain of SYNJ1 identified mutated in a family with early-onset progressive Parkinsonism with generalized seizures. Human Mutation, 34(9), pp. 1200-1207.

Kumar, V., Sami, N., Kashav, T., Islam, A., Ahmad, F., \& Hassan, M. I. (2016). Protein aggregation and neurodegenerative diseases: From theory to therapy. Eur $J$ Med Chem., 124, pp. 1105-1120.

Kumari, U., \& Tan, E. K. (2009). LRRK2 in Parkinson's disease: genetic and clinical studies from patients. FEBS J, 276(22), pp. 6455-6463.

Kundu, M., \& Thompson, C. B. (2008). Autophagy: basic principles and relevance to disease. Annu Rev Pathol., 3, pp. 427-455.

Kunz, J. B., Schwarz, H., \& Mayer, A. (2004). Determination of four sequential stages during microautophagy in vitro. J Biol. Chem., 279(11), pp. 9987-9996.

Lagirand-Cantaloube, J., Offner, N., Csibi, A., Leibovitch, M. P., Batonnet-Pichon, S., Tintignac, L. A., . . Leibovitch, S. A. (2008). The initiation factor elF3-f is a major target for atrogin1/MAFbx function in skeletal muscle atrophy. EMBO J, 27(8), pp. 1266-1276.

Lee, D. H., \& Goldberg, A. L. (1996). Selective inhibitors of the proteasomedependent and vacuolar pathways of protein degradation in Saccharomyces cerevisiae. J Biol Chem., 271(44), pp. 27280-27284.

Lee, D. W., Wu, X., Eisenberg, E., \& Greene, L. E. (2006). Recruitment dynamics of GAK and auxilin to clathrin-coated pits during endocytosis. J Cell Sci., 119(Pt 17), pp. 3502-3512.

Lee, G., \& Leugers, C. J. (2012). Tau and tauopathies. Prog Mol Biol Trans/ Sci., 107, pp. 263-293.

Lee, M. K., Kim, Y. M., Kim, I. H., Choi, Y. H., \& Nam, T. J. (2017). Pyropia yezoensis peptide PYP1-5 protects against dexamethasone-induced muscle atrophy through the downregulation of atrogin1/MAFbx and MuRF1 in mouse C2C12 myotubes. Mol Med Rep., 15(6), pp. 3507-3514.

Levine, B., \& Kroemer, G. (2008). Autophagy in the pathogenesis of disease. Cell, 132(1), pp. 27-42.

Li, H. H., Kedar, V., Zhang, C., McDonough, H., Arya, R., Wang, D. Z., \& Patterson, C. (2004). Atrogin-1/muscle atrophy F-box inhibits calcineurin-dependent cardiac hypertrophy by participating in an SCF ubiquitin ligase complex. $J$ Clin Invest., 114(8), pp. 1058-1071.

Li, Z., Zhao, J., Li, Q., Yang, W., Song, Q., Li, W., \& Liu, J. (2010). KLF4 promotes hydrogen-peroxide-induced apoptosis of chronic myeloid leukemia cells involving the bcl-2/bax pathway. Cell Stress Chaperones, 15(6), pp. 905-912. 
Liang, C., Feng, P., Ku, B., Dotan, I., Canaani, D., Oh, B. H., \& U, J. J. (2006). Autophagic and tumour suppressor activity of a novel Beclin1-binding protein UVRAG. Nat Cell Biol., 8(7), pp. 688-699.

Liang, Y., \& Sigrist, S. (2018). Autophagy and proteostasis in the control of synapse aging and disease. Current Opinion in Neurobiology(48), pp. 113-121.

Liu, C. H., Goldberg, A. L., \& Qiu, X. B. (2007). New insights into the role of the ubiquitin-proteasome pathway in the regulation of apoptosis. Chang Gung Med J, 30(6), pp. 469-479.

Lokireddy, S., Wijesoma, I. W., Sze, S. K., McFarlane, C., Kambadur, R., \& Sharma, M. (2012). Identification of atrogin-1-targeted proteins during the myostatininduced skeletal muscle wasting. Am J Physiol Cell Physiol., 303(5), pp. C512529.

Malecz, N., McCabe, P. C., Spaargaren, C., Qiu, R.-G., Chuang, Y.-Y., \& Symons, M. (2000). Synaptojanin 2, a novel Rac1 effector that regulates clathrin-mediated endocytosis. Brief Comm, 10(21), pp. 1383-1386.

Mandillo, S., Tucci, V., Hölter, S. M., Meziane, H., Banchaabouchi, M. A., Kallnik, M., ... Wurst, W. (2008). Reliability, robustness, and reproducibility in mouse behavioral phenotyping: a cross-laboratory study. Physiol Genomics, 34(3), pp. 243-255.

Mani, M., Lee, S. Y., Lucast, L., Cremona, O., Di Paolo, G., De Camilli, P., \& Ryan, T. A. (2007). The dual phosphatase activity of synaptojanin1 is required for both efficient synaptic vesicle endocytosis and reavailability at nerve terminals. Neuron, 56(6), 1004-1018.

Marchenko, S., \& Flanagan, L. (2007). Immunocytochemistry: human neural stem cells. J Vis Exp., 7, p. e267.

Martin, G. M., Austad, S. N., \& Johnson, T. E. (1996). Genetic analysis of ageing: role of oxidative damage and environmental stresses. Nat Genet., 13(1), pp. 25-34.

Martinez-Vicente, M., Sovak, G., \& Cuervo, A. M. (2005). Protein degradation and aging. Exp Gerontol., 40(8-9), pp. 622-633.

Massol, R. H., Boll, W., Griffin, A. M., \& Kirchhausen, T. (2006). A burst of auxilin recruitment determines the onset of clathrin-coated vesicle uncoating. Proc Natl Acad Sci U S A, 103(27), pp. 10265-10270.

Masuda, M., Takeda, S., Sone, M., Ohki, T., Mori, H., Kamioka, Y., \& Mochizuki, N. (2006). Endophilin BAR domain drives membrane curvature by two newly identified structure-based mechanisms. EMBO J., 25(12), pp. 2889-2897.

Matsunaga, K., Saitoh, T., Tabata, K. O., Satoh, T., Kurotori, N., Maejima, I., . . . Yoshimori, T. (2009). Two Beclin 1-binding proteins, Atg14L and Rubicon, reciprocally regulate autophagy at different stages. Nat Cell Biol., 11(4), pp. 385-396.

Matsuzaki, M., Ellis-Davies, G. C., Nemoto, T., Miyashita, Y., lino, M., \& Kasai, H. (2001). Dendritic spine geometry is critical for AMPA receptor expression in hippocampal CA1 pyramidal neurons. Nature Neuroscience, 4(11), pp. 10861092.

Matsuzaki, M., Honkura, N., Ellis-Davies, G. C., \& Kasai, H. (2004). Structural basis of long-term potentiation in single dendritic spines. Nature, 429(6993), pp. 761766. 
Matta, S., Van Kolen, K., da Cunha, R., Van den Gogaart, G., Mandemakers, W., Miskiewicz, K., . . . Verstreken, P. (2012). LRRK2 Controls an EndoA Phosphorylation Cycle in Synaptic Endocytosis. Neuron, 75(6), pp. 1008-1021.

Mayor, S., Parton, R. G., \& Donaldson, J. G. (2014). Clathrin-Independent Pathways of Endocytosis. Cold Spring Harb Perspect Biol., 6(6), p. a016758.

McKinney, R. A. (2010). Excitatory amino acid involvement in dendritic spine formation, maintenance and remodelling. J Physiol., 588(1), pp. 107-116.

McPherson, P. S., Garcia, E. P., Slepnev, V. I., David, C., Zhang, X., Grabs, D., . . . De Camilli, P. (1996). A presynaptic inositol-5-phosphatase. Nature, 379(6563), pp. 353-357.

McPherson, P. S., Ritter, B., \& Wendland, B. (2000-2013). Clathrin-Mediated Endocytosis. (M. C. [Internet], Ed.) Austin (TX): Landes Bioscience.

McPherson, P. S., Takei, K., Schmid, S. L., \& De Camilli, P. (1994). p145, a major Grb2-binding protein in brain, is co-localized with dynamin in nerve terminals where it undergoes activity-dependent dephosphorylation. J Biol Chem, 269, pp. 30132-30139.

Mei, Z., Zhang, D., Hu, B., Wang, J., Shen, X., \& Xiao, W. (2015). FBXO32 Targets C-Myc for Proteasomal Degradation and Inhibits c-Myc Activity. J Biol Chem., 290(26), pp. 16202-16214.

Meinecke, M., Boucrot, E., Camdere, G., Hon, W. C., Mittal, R., \& McMahon, H. T. (2013). Cooperative recruitment of dynamin and BIN/amphiphysin/Rvs (BAR) domain-containing proteins leads to GTP-dependent membrane scission. $J$ Biol Chem., 288(9), 6651-6661.

Menzies, F. M., Fleming, A., \& Rubinsztein, D. C. (2015). Compromised autophagy and neurodegenerative diseases. Nat Rev Neurosci., 16(6), pp. 345-357.

Meshram, S. N., Paul, D., Manne, R., Choppara, S., Sankaran, G., Agrawal, Y., \& Santra, M. K. (2017). FBXO32 activates NF-KB through IKBa degradation in inflammatory and genotoxic stress. International Journal of Biochemistry and Cell Biology(92), pp. 134-140.

Metzger, M. B., Pruneda, J. N., Klevit, R. E., \& Weissman, A. M. (2014). RING-type E3 ligases: master manipulators of E2 ubiquitin-conjugating enzymes and ubiquitination. Biochim Biophys Acta, 1843(1), pp. 47-60.

Micheva, K. D., Kay, B. K., \& McPherson, P. S. (1997). Synaptojanin Forms Two Separate Complexes in the Nerve Terminal. J Biol. Chem., 272, 27239-27245.

Miller, T. M., \& Heuser, J. E. (1984). Endocytosis of synaptic vesicle membrane at the frog neuromuscular junction. J Cell Biol., 98(2), pp. 685-698.

Milosevic, I., Giovedi, S., Raimondi, A., Collesi, C., Shen, H., Paradise, S., . . . De Camilli, P. (2011). Recruitment of endophilin to clathrin-coated pit necks is required for efficient vesicle uncoating after fission. Neuron, 72(4), pp. 587601.

Mincheva-Tasheva, S., \& Soler, R. M. (2013). NF-KB signaling pathways: role in nervous system physiology and pathology. Neuroscientist, 19(2), pp. 175-194.

Modregger, J., Schmidt, A. A., Ritter, B., Huttner, W. B., \& Plomann, M. (2003). Characterization of Endophilin B1b, a brain-specific membrane-associated lysophosphatidic acid acyl transferase with properties distinct from endophilin A1. J Biol Chem., 278(6), pp. 4160-4167. 
Mukherjee, A., Patel, B., Koga, H., Cuervo, A. M., \& Jenny, A. (2016). Selective endosomal microautophagy is starvation-inducible in Drosophila. Autophagy, 12(11), pp. 1984-1999.

Murdoch, J. D., Rostosky, C. M., Gowrisankaran, S., Arora, A. S., Soukup, S. F., Vidal, R., . . . Milosevic, I. (2016). Endophilin-A Deficiency Induces the Foxo3aFbxo32 Network in the Brain and Causes Dysregulation of Autophagy and the Ubiquitin-Proteasome System. Cell Reports, 17(4), 1071-1086.

Nayak, D., Roth, T. L., \& McGavern, D. B. (2014). Microglia Development and Function. Annual Review of Immunology(32), pp. 367-402.

Nemoto, Y., \& De Camilli, P. (1999). Recruitment of an alternatively spliced form of synaptojanin 2 to mitochondria by the interaction with the PDZ domain of a mitochondrial outer membrane protein. EMBO J, 18(11), pp. 2991-3006.

Nemoto, Y., Arribas, M., Haffner, C., \& De Camilli, P. (1997). Synaptojanin 2, a Novel Synaptojanin Isoform with a Distinct Targeting Domain and Expression Pattern. J Biol Chem, 272, pp. 30817-30821.

Newmyer, S. L., Christensen, A., \& Sever, S. (2003). Auxilin-dynamin interactions link the uncoating ATPase chaperone machinery with vesicle formation. Dev Cell, 4(6), pp. 929-940.

Nichols, W. C., Pankratz, N., Hernandez, D., Paisán-Ruíz, C., Jain, S., Halter, C. A., ... Parkinson Study Group-PROGENI investigators. (2005). Genetic screening for a single common LRRK2 mutation in familial Parkinson's disease. Lancet, 365(9457), pp. 410-412.

Nixon, R. A. (2013). The role of autophagy in neurodegenerative disease. Nature Medicine, 19(8), pp. 983-97.

Novack, J. P. (2017). Autophagy and Proteostasis: A Unifying Theory of Neurodegenerative Disease. J Rest Med., 6, pp. 3-18.

Okamoto, K., \& Kondo-Okamoto, N. (2012). Mitochondria and autophagy: critical interplay between the two homeostats. Biochim Biophys Acta, 1820(5), pp. 595-600.

Olgiati, S., De Rosa, A., Quadri, M., Criscuolo, C., Breedveld, G. J., Picillo, M., . . . Bonifati, V. (2014). PARK20 caused by SYNJ1 homozygous Arg258Gin mutation in a new Italian family. Neurogenetics, 15(3), pp. 183-188.

Olgiati, S., Quadri, M., Fang, M., Rood, J. P., Saute, J. A., Chien, H. F., . . Bonifati, V. (2016). DNAJC6 Mutations Associated With Early-Onset Parkinson's Disease. Ann Neurol., 79(2), pp. 244-256.

Park, R. J., Shen, H., Liu, L., Liu, X., Ferguson, S. M., \& De Camilli, P. (2013). Dynamin triple knockout cells reveal off target effects of commonly used dynamin inhibitors. J Cell Sci., 126(Pt 22), pp. 5305-5312.

Pattingre, S., Tassa, A., Qu, X., Garuti, R., Liang, X. H., Mizushima, N., . . Levine, B. (2005). Bcl-2 antiapoptotic proteins inhibit Beclin 1-dependent autophagy. Cell, 122(6), pp. 927-939.

Pickart, C. M., \& Fushman, D. (2004). Polyubiquitin chains: polymeric protein signals. Curr Opin Chem Biol, 8(6), pp. 610-616.

Pierrat, B., Simonen, M., Cueto, M., Mestan, J., Ferrigno, P., \& Heim, J. (2001). SH3GLB, a new endophilin-related protein family featuring an SH3 domain. Genomics, 71(2), pp. 222-234. 
Piper, R. C., \& Luzio, J. P. (2001). Late endosomes: sorting and partitioning in multivesicular bodies. Traffic, 2(9), pp. 612-621.

Pirruccello, M., \& De Camilli, P. (2013). The Inositol 5-phosphatases: insights from the Lowe Syndrome Protein OCRL. Trends Biochem Sci., 37(4), pp. 134-143.

Porter, A. G., \& Jänicke, R. U. (1999). Emerging roles of caspase-3 in apoptosis. Cell Death Differ., 6(2), pp. 99-104.

Powell, E., Anch, A. M., Dyche, J., Bloom, C., \& Richter, R. R. (1999). The Splay Angle: A New Measure for Assessing Neuromuscular Dysfunction in Rats. Physiol Behav, 67(5), pp. 819-821.

Puri, C., Renna, M., Bento, C. F., Moreau, K., \& Rubinsztein, D. C. (2014). ATG16L1 meets ATG9 in recycling endosomes: additional roles for the plasma membrane and endocytosis in autophagosome biogenesis. Autophagy, 10(1), pp. 182-184.

Quadri, M., Fang, M., Picillo, M., Olgiati, S., Breedveld, G. J., Graafland, J., . . . Bonifati, V. (2013). Mutation in the SYNJ1 gene associated with autosomal recessive, early-onset Parkinsonism. Human Mutation, 34(9), pp. 1208-1215.

Raimondi, A., Ferguson, S. M., Lou, X., Armbruster, M., Paradise, S., Giovedi, S., . . . De Camilli, P. (2011). Overlapping role of dynamin isoforms in synaptic vesicle endocytosis. Neuron, 70(6), pp. 1100-1114.

Ralser, M., Nonhoff, U., Albrecht, M., Lengauer, T., Wanker, E. E., Lehrach, H., \& Krobitsch, S. (2005). Ataxin-2 and huntingtin interact with endophilin-A complexes to function in plastin-associated pathways. Hum Mol Gen, 14(19), pp. 2893-2909.

Ramón-Moliner, E. (1970). The Golgi-Cox Technique. In E. Ramón-Moliner, Contemporary Research Methods in Neuroanatomy (pp. 32-55). Berlin, Heidelberg: Springer.

Ravikumar, B., Moreau, K., Jahreiss, L., Puri, C., \& Rubinsztein, D. C. (2010). Plasma membrane contributes to the formation of pre-autophagosomal structures. Nat Cell Biol., 12(8), pp. 747-757.

Reggiori, F. (2006). Membrane origin for autophagy. Curr Top Dev Biol., 74, pp. 1-30. Ren, G., Vajjhala, P., Lee, J. S., Winsor, B., \& Munn, A. L. (2006). The BAR Domain Proteins: Molding Membranes in Fission, Fusion, and Phagy. Microbiol. Mol. Biol. Rev., 70(1), pp. 37-120.

Ren, Y., Xu, H. W., Davey, F., Taylor, M., Aiton, J., Coote, P., . . Gunn-Moore, F. J. (2008). Endophilin I expression is increased in the brains of Alzheimer disease patients. J Biol Chem., 283(9), pp. 5685-5691.

Reubold, T. F., Faelber, K., Plattner, N., Posor, Y., Ketel, K., Curth, U., . . . Eschenburg, S. (2015). Crystal structure of the dynamin tetramer. Nature, 525(7569), pp. 404-408.

Richards, D. A., Guatimosim, C., \& Betz, W. J. (2000). Two endocytic recycling routes selectively fill two vesicle pools in frog motor nerve terminals. Neuron, 27(3), pp. 551-559.

Ringstad, N., Gad, H., Löw, P., Di Paolo, G., Brodin, L., Shupliakov, O., \& De Camilli, P. (1999). Endophilin/SH3p4 is required for the transition from early to late stages in clathrin-mediated synaptic vesicle endocytosis. Neuron, 24(1), 143154. 
Ringstad, N., Nemoto, Y., \& De Camilli, P. (1997). The SH3p4/Sh3p8/SH3p13 protein family: Binding partners for synaptojanin and dynamin via a Grb2-like Src homology 3 domain. Proc Natl Acad Sci USA, 94(16), pp. 8569-8574.

Rizzoli, S. O. (2014). Synaptic vesicle recycling: steps and principles. EMBO J., 33(8), pp. 788-822.

Rizzoli, S. O., \& Jahn, R. (2007). Kiss-and-run, collapse and 'readily retrievable' vesicles. Traffic, 8(9), pp. 1137-1144.

Rochefort, N. L., \& Konnerth, A. (2012). Dendritic spines: from structure to in vivo function. EMBO Rep., 13(8), pp. 699-708.

Rock, K. L., Gramm, C., Rothstein, L., Clark, K., Stein, R., Dick, L., . . Goldberg, A. L. (1994). Inhibitors of the proteasome block the degradation of most cell proteins and the generation of peptides presented on MHC class I molecules. Cell, 78(5), pp. 761-771.

Rosenzweig, R., Osmulski, P. A., Gaczynska, M., \& Glickman, M. H. (2008). The central unit within the $19 \mathrm{~S}$ regulatory particle of the proteasome. Nat Struct Mol Biol., 15(6), pp. 573-580.

Rostosky, C. M., \& Milosevic, I. (2018). Gait Analysis of Age-dependent Motor Impairments in Mice with Neurodegeneration. J. Vis. Exp., 136, p. e57752.

Rubinsztein, D. C., Mariño, G., \& Kroemer, G. (2011). Autophagy and aging. Cell, 146(5), pp. 682-695.

Sadowski, M., Suryadinata, R., Tan, A. R., Roesley, S. N., \& Sarcevic, B. (2012). Protein monoubiquitination and polyubiquitination generate structural divers ity to control distinct biological processes. IUBMB Life, 64(2), pp. 136-142.

Sahu SK1, T. N., Zhuang, Y., Borisova, M., Diken, M., Strand, S., Beli, P., \& Tiwari, V. K. (2017). FBXO32 promotes microenvironment underlying epithelialmesenchymal transition via CtBP1 during tumour metastasis and brain development. Nat Commun, 8(1), p. e1523.

Sahu, R., Kaushik, S., Clement, C. C., Cannizzo, E. S., Scharf, B., Follenzi, A., . . . Santambrogio, L. (2011). Microautophagy of cytosolic proteins by late endosomes. Dev Cell, 20(1), pp. 131-139.

Sakai, H., Kimura, M., Isa, Y., Yabe, S., Maruyama, A., Tsuruno, Y., . . Narita, M. (2017). Effect of acute treadmill exercise on cisplatin-induced muscle atrophy in the mouse. Pflugers Arch., 469(11), pp. 1495-1501.

Salvador, N., Aguado, C., Horst, M., \& Knecht, E. (2000). Import of a cytosolic protein into lysosomes by chaperone-mediated autophagy depends on its folding state. J Biol Chem., 275(35), pp. 27447-27456.

Sandri, M., Sandri, C., Gilbert, A., Skurk, C., Calabria, E., Picard, A., . . Goldberg, A. L. (2004). Foxo transcription factors induce the atrophy-related ubiquitin ligase atrogin-1 and cause skeletal muscle atrophy. Cell, 117(3), pp. 399-412.

Sanpei, K., Takano, H., Igarashi, S., Sato, T., Oyake, M., Sasaki, H., . . . Tsuji, S. (1996). Identification of the spinocerebellar ataxia type 2 gene using a direct identification of repeat expansion and cloning technique, DIRECT. Net Genet., 14(3), pp. 277-284.

Sassoon, D., Lyons, G., Wright, W. E., Lin, V., Lassar, A., Weintraub, H., \& Buckingham, M. (1989). Expression of two myogenic regulatory factors myogenin and MyoD1 during mouse embryogenesis. Nature, 341(6240), pp. 303-307. 
Sato, S., Uchihara, T., Fukuda, T., Noda, S., Kondo, H., Saiki, S., . . Hattori, N. (2018). Loss of autophagy in dopaminergic neurons causes Lewy pathology and motor dysfunction in aged mice. Sci Rep., 8(1), e2813.

Scheele, U., Kalthoff, C., \& Ungewickell, E. (2001). Multiple interactions of auxilin 1 with clathrin and the AP-2 adaptor complex. J Biol. Chem., 276(39), pp. 3613136138.

Schikorski, T., \& Stevens, C. F. (1997). Quantitative Ultrastructural Analysis of Hippocampal Excitatory Synapses. J Neuroscience, 17(15), pp. 5858-5867.

Schirinzi, T., Madeo, G., Martella, G., Maltese, M., Picconi, B., Calabresi, P., \& Pisani, A. (2016). Early synaptic dysfunction in Parkinson's disease: Insights from animal models. Mov Disord., 31(6), pp. 802-813.

Schreiner, P., Chen, X., Husnjak, K., Randles, L., Zhang, N., Elsasser, S., . . Groll, M. (2008). Ubiquitin docking at the proteasome through a novel pleckstrinhomology domain interaction. Nature, 453(7194), pp. 548-552.

Shi, M., Bradner, J., Bammler, T. K., Eaton, D. L., Zhang, J. P., Ye, Z. C., . . Zhang, J. (2009). Identification of Glutathione S-Transferase Pi as a Protein Involved in Parkinson Disease Progression. Am J Path., 175(1), pp. 54-65.

Silva, G. M., Finley, D., \& Vogel, C. (2015). K63 polyubiquitination is a new modulator of the oxidative stress response. Nature Struct. Mol. Biol., 22(2), pp. 116-123.

Simonsen, A., \& Tooze, S. A. (2009). Coordination of membrane events during autophagy by multiple class III PI3-kinase complexes. J Cell Biol., 186(6), pp. 773-782.

Skowyra, D., Craig, K. L., Tyers, M., Elledge, S. J., \& Harper, J. W. (1997). F-box proteins are receptors that recruit phosphorylated substrates to the SCF ubiquitin-ligase complex. Cell, 91(2), pp. 209-219.

Skowyra, D., Craig, K. L., Tyers, M., Elledge, S. J., \& Harper, J. W. (1997). F-box proteins are receptors that recruit phosphorylated substrates to the SCF ubiquitin-ligase complex. Cell, 91(2), pp. 209-219.

Sluimer, J., \& Distel, B. (2018). Regulating the human HECT E3 ligases. Cell Mol Life Sci., 75(17), pp. 3121-3141.

So, C. W., Sham, M. H., Chew, S. L., Cheung, N., So, C. K., Chung, S. K., . . Chan, L. C. (2000). Expression and protein-binding studies of the EEN gene family, new interacting partners for dynamin, synaptojanin and huntingtin proteins. Biochem J., 348(2), 447-458.

Soukup, S. F., Kuenen, S., Vanhauwaert, R., Manetsberger, J., Hernández-Díaz, S., Swert, s. J., . . . Verstreken, P. (2016). A LRRK2-Dependent EndophilinA Phosphoswitch is Critical for Macroautophagy at Presynaptic Terminals. Neuron, 92(4), pp. 829-844.

Soukup, S. F., Vanhauwaert, R., \& Verstreken, P. (2018). Parkinson's disease: convergence on synaptic homeostasis. EMBO J, 37(15), e98960.

Südhof, T. C. (2012). The Presynaptic Active Zone. Neuron, 75(1), 11-25.

Sui, X., Kong, N., Ye, L., Han, W., Zhou, J., Zhang, Q., . . Pan, H. (2014). p38 and JNK MAPK pathways control the balance of apoptosis and autophagy in response to chemotherapeutic agents. Cancer Letter, 344(2), pp. 174-179.

Sundborger, A. C., \& Hinshaw, J. E. (2014). Regulating dynamin dynamics during endocytosis. F1000Prime Rep., 6(85). 
Suzuki, K., \& Ohsumi, Y. (2010). Current knowledge of the pre-autophagosomal structure (PAS). FEBS Lett., 584(7), pp. 1280-1286.

Suzuki, K., Kirisako, T., Kamada, Y., Mizushima, N., Noda, T., \& Ohsumi, Y. (2001). The pre-autophagosomal structure organized by concerted functions of APG genes is essential for autophagosome formation. EMBO J, 20(21), pp. 59715981.

Takahashi, Y., Coppola, D., Matsushita, N., Cualing, H. D., Sun, M., Sato, Y., . . . Wang, H. G. (2007). Bif-1 interacts with Beclin 1 through UVRAG and regulates autophagy and tumorigenesis. Nat Cell Biol., 9(10), pp. 1142-1151.

Takei, K., Mundigl, O., Daniell, L., \& De Camilli, P. (1996). The synaptic vesicle cycle: a single vesicle budding step involving clathrin and dynamin. J Cell Biol., 133(6), pp. 1237-1250.

Takeshige, K., Baba, M., Tsuboi, S., Noda, T., \& Ohsumi, Y. (1992). Autophagy in yeast demonstrated with proteinase-deficient mutants and conditions for its induction. J Cell Biol, 119(2), pp. 301-311.

Tanaka. (2009). The proteasome: overview of structure and functions. Proc Jpn Acad Ser B Phys Biol Sci., 85(1), pp. 12-36.

Tanaka, Y., Guhde, G., Suter, A., Eskelinen, E. L., Hartmann, D., Lüllmann-Rauch, R., . . . Saftig, P. (2000). Accumulation of autophagic vacuoles and cardiomyopathy in LAMP-2-deficient mice. Nature, 406(6798), pp. 902-906.

Taneike, M., Yamaguchi, O., Nakai, A., Hikoso, S., Takeda, T., Mizote, I., . . O Otsu, K. (2010). Inhibition of autophagy in the heart induces age-related cardiomyopathy. Autophagy, 6(5), pp. 600-606.

Tekirdag, K. A., \& Cuervo, A. M. (2017). Chaperone-mediated autophagy and endosomal microautophagy: joint by a chaperone. J Biol Chem., 293(15), pp. 5414-5424.

Tekirdag, K., \& Cuervo, A. M. (2017). Chaperone-mediated autophagy and endosomal microautophagy: joint by a chaperone. Journal Biological Chemistry.

Tintignac, L. A., Lagirand, J., Batonnet, S., Sirri, V., Leibovitch, M. P., \& Leibovitch, S. A. (2005). Degradation of MyoD mediated by the SCF (MAFbx) ubiquitin ligase. J Biol Chem., 280(4), pp. 2847-2856.

Totta, P., Busonero, C., Leone, S., Marino, M., \& Acconcia, F. (2016). Dynamin II is required for $17 \beta$-estradiol signaling and autophagy-based ERa degradation. Sci Rep., 24(6), p. e23727.

Trempe, J. K., Chen, C. X., Grenier, K., Camacho, E. M., Kozlov, G., McPherson, P. S., . . Fon, E. A. (2009). SH3 domains from a subset of BAR proteins define a Ubl-binding domain and implicate parkin in synaptic ubiquitination. Mol Cell, 36(6), pp. 1034-1047.

Tsutsui, K., Maeda, Y., Tsutsui, K., Seki, S., \& Tokunaga, A. (1997). cDNA Cloning of a Novel Amphiphysin Isoform and Tissue-Specific Expression of Its Multiple Splice Variants. Biochem. Biophys. Res. Comm., 236(1), pp. 178-183.

Uttenweiler, A., Schwarz, H., Neumann, H., \& Mayer, A. (2007). The vacuolar transporter chaperone (VTC) complex is required for microautophagy. Mol Biol Cell, 18(1), pp. 166-175. 
Valimberti, I., Tiberti, M., Lambrughi, M., Sarcevic, B., \& Papaleo, E. (2015). E2 superfamily of ubiquitin-conjugating enzymes: constitutively active or activated through phosphorylation in the catalytic cleft. Sci Rep., 5, p. e14849.

Vanhauwaert, R., Kuenen, S., Masius, R., Bademosi, A., Manetsberger, J., Schoovaerts, N., .. Verstreken, P. (2017). The SAC1 domain in synaptojanin is required for autophagosome maturation at presynaptic terminals. EMBO J, 36(10), pp. 1392-1411.

Ventii, K. H., \& Wilkinson, K. D. (2008). Protein partners of deubiquitinating enzymes. Biochem J, 414(2), pp. 161-175.

Verstreken, P., Koh, T.-W., Schulze, K. L., Zhai, R. G., Hiesinger, P. R., Zhou, Y., . . . Bellen, H. J. (2003). Synaptojanin Is Recruited by Endophilin to Promote Synaptic Vesicle Uncoating. Neuron, 40(4), pp. 733-748.

Voglis, G., \& Tavernarakis, N. (2006). The role of synaptic ion channels in synaptic plasticity. EMBO Rep., 7(11), pp. 1104-1110.

Wang, D., \& Hiesinger, P. R. (2012). Autophagy, neuron-specific degradation and neurodegeneration. Autophagy, 8(4), pp. 711-713.

Watanabe, S., \& Boucrot, E. (2017). Fast and ultrafast endocytosis. Curr Opin Cell Biol., 47, pp. 64-71.

Watanabe, S., Mamer, L. E., Raychaudhuri, S., Luvsanjav, D., Eisen, J., Trimbuch, T., ... Jorgensen, E. M. (2018). Synaptojanin and Endophilin Mediate Neck Formation during Ultrafast Endocytosis. Neuron, 98(6), pp. 1184-1197.

Watanabe, S., Rost, B. R., Camacho-Pérez, M., Davis, M. W., Söhl-Kielczynski, B., Rosenmund, C., \& Jorgensen, E. M. (2013). Ultrafast endocytosis at mouse hippocampal synapses. Nature, 504(7479), pp. 242-247.

Watanabe, S., Trimbuch, T., Camacho-Pérez, M., Rost, B. R., Brokowski, B., SöhlKielczynski, B., . . . Jorgensen, E. M. (2014). Clathrin regenerates synaptic vesicles from endosomes. Nature, 515(7526), pp. 228-233.

Wegener, E., Brendel, C., Fischer, A., Hülsmann, S., Gärtner, J., \& Huppke, P. (2014). Characterization of the MeCP2R168X knockin mouse model for Rett syndrome. PLoS One, 9(12), p. e115444.

Weissenhorn, W. (2005). Crystal structure of the endophilin-A1 BAR domain. J Mol Biol., 351(3), pp. 653-661.

Wenzel, H. J., Hunsaker, M. R., Greco, C. M., Willemsen, R., \& Berman, R. F. (2010). Ubiquitin-positive intranuclear inclusions in neuronal and glial cells in a mouse model of the fragile X premutation. Brain Res., 1318, pp. 155-166.

Wienisch, M., \& Klingauf, J. (2006). Vesicular proteins exocytosed and subsequently retrieved by compensatory endocytosis are nonidentical. Nat Neuroscience, 9(8), pp. 1019-1027.

Willis, M. S., \& Patterson, C. (2006). Into the heart: the emerging role of the ubiquitinproteasome system. J Mol Cell Cardiol., 41(4), pp. 567-579.

Winston, J. T., Strack, P., Beer-Romero, P., Chu, C. Y., Elledge, S. J., \& Harper, J. W. (1999). The SCFbeta-TRCP-ubiquitin ligase complex associates specifically with phosphorylated destruction motifs in IkappaBalpha and betacatenin and stimulates IkappaBalpha ubiquitination in vitro. Genes Dev, 13(3), pp. 270-283.

Woolfrey, K. M., \& Srivastava, D. P. (2016). Control of Dendritic Spine Morphological and Functional Plasticity by Small GTPases. Neural Plasticity, 2016:3025948. 
Wu, X. S., McNeil, B. D., Xu, J., Fan, J., Xue, L., Melicoff, E., . . Wu, L. G. (2009). $\mathrm{Ca}(2+)$ and calmodulin initiate all forms of endocytosis during depolarization at a nerve terminal. Nature Neuroscience, 12(8), pp. 1003-1010.

Wu, Y., Matsui, H., \& Tomizawa, K. (2009). Amphiphysin I and Regulation of Synaptic Vesicle Endocytosis. Acta Med Okayama, 63(6), pp. 305-323.

Xie, P., Guo, S., Fan, Y., Zhang, H., Gu, D., \& Li, H. (2009). Atrogin-1/MAFbx enhances simulated ischemia/reperfusion-induced apoptosis in cardiomyocytes through degradation of MAPK phosphatase-1 and sustained JNK activation. J Biol Chem., 284(9), pp. 5488-5496.

Xie, Z., \& Klionsky, D. J. (2007). Autophagosome formation: core machinery and adaptations. Nat Cell Biol., 9(10), pp. 1102-1109.

Yim, Y. I., Sun, T., Wu, L. G., Raimondi, A., De Camilli, P., Eisenberg, E., \& Greene, L. E. (2010). Endocytosis and clathrin-uncoating defects at synapses of auxilin knockout mice. Proc Natl Acad Sci U S A, 107(9), pp. 4412-4417.

Ylä-Anttila, P., Vihinen, H., Jokitalo, E., \& Eskelinen, E. L. (2009). 3D tomography reveals connections between the phagophore and endoplasmic reticulum. Autophagy, 5(8), pp. 1180-1185.

Zaglia, T., Milan, G., Ruhs, A., Franzoso, M., Bertaggia, E., Pianca, N., . . Sandri, M. (2014). Atrogin-1 deficiency promotes cardiomyopathy and premature death via impaired autophagy. J Clinical Invest., 124(6), pp. 2410-2424.

Zheng, N., \& Shabek, N. (2017). Ubiquitin Ligases: Structure, Function, and Regulation. Annu Rev Biochem., 86, pp. 129-157.

Zhou, H., Liu, Y., Zhu, R., Ding, F., Wan, Y., Li, Y., \& Liu, Z. (2017). FBXO32 suppresses breast cancer tumorigenesis through targeting KLF4 to proteasomal degradation. Oncogene, 36(23), pp. 3312-3321.

Zuchero, J. B., \& Barres, B. A. (2015). Glia in mammalian development and disease. Development, 142(22), pp. 3805-3809. 
Appendix 


\section{Supplemental figures}
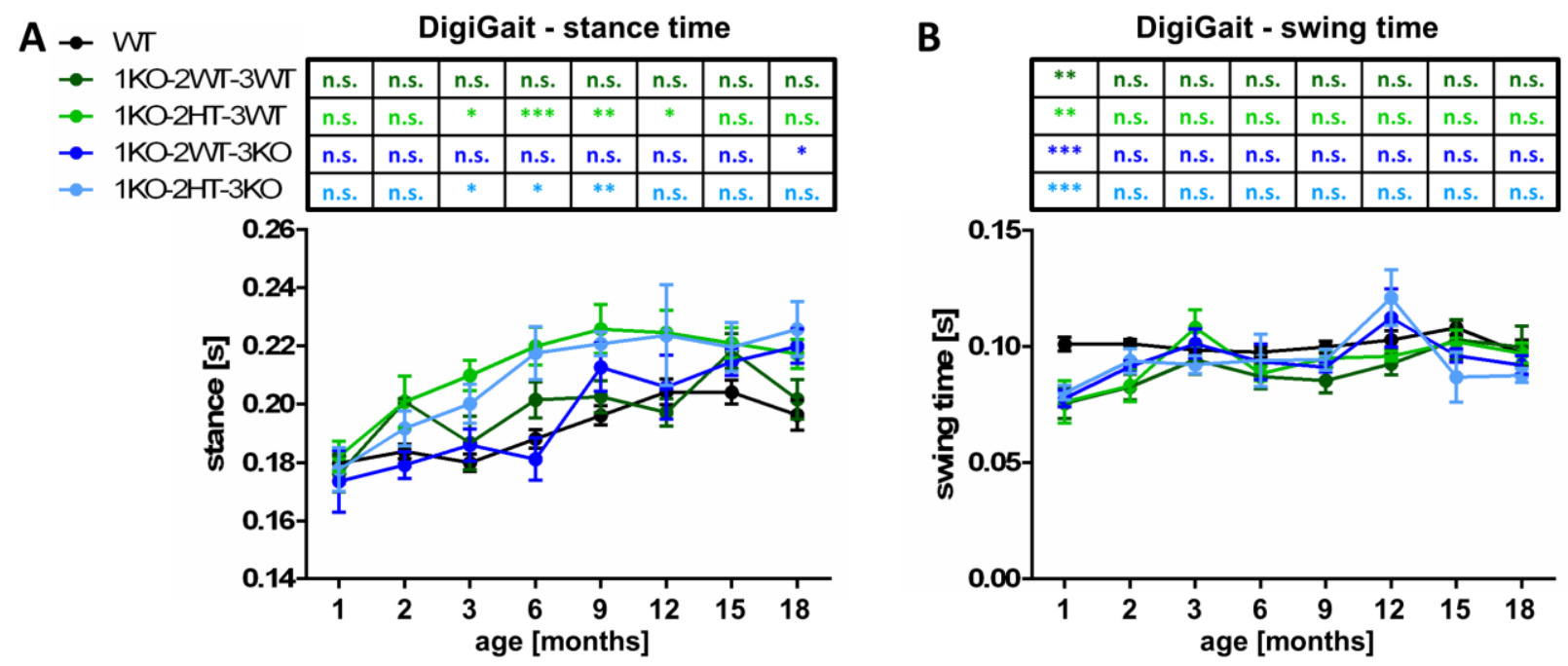

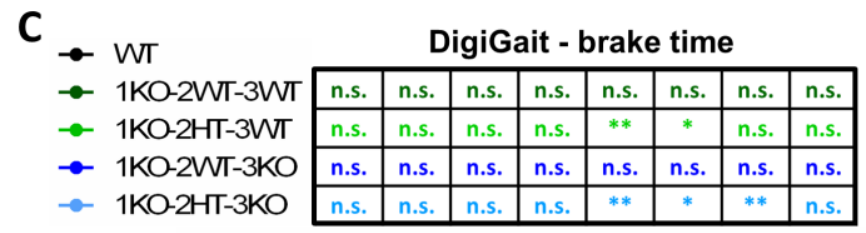

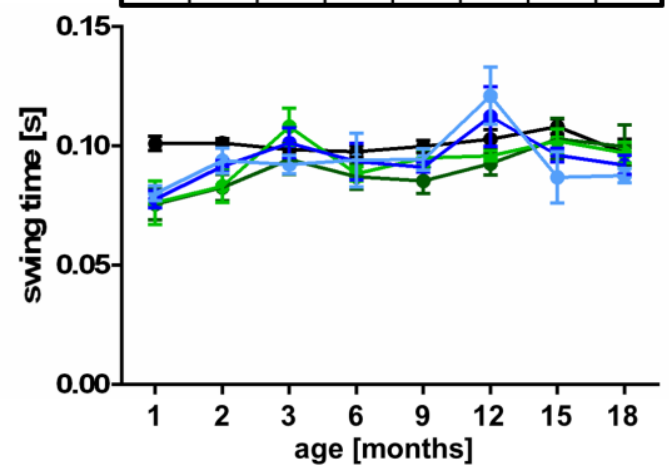

D

DigiGait - propel time
\begin{tabular}{|l|l|l|l|l|l|l|l|}
\hline n.s. & n.s. & n.s. & n.s. & n.s. & n.s. & n.s. & n.s. \\
\hline n.s. & n.s. & n.s. & n.s. & n.s. & n.s. & n.s. & n.s. \\
\hline n.s. & n.s. & n.s. & n.s. & n.s. & n.s. & n.s. & n.s. \\
\hline n.s. & n.s. & n.s. & n.s. & n.s. & n.s. & n.s. & n.s. \\
\hline
\end{tabular}
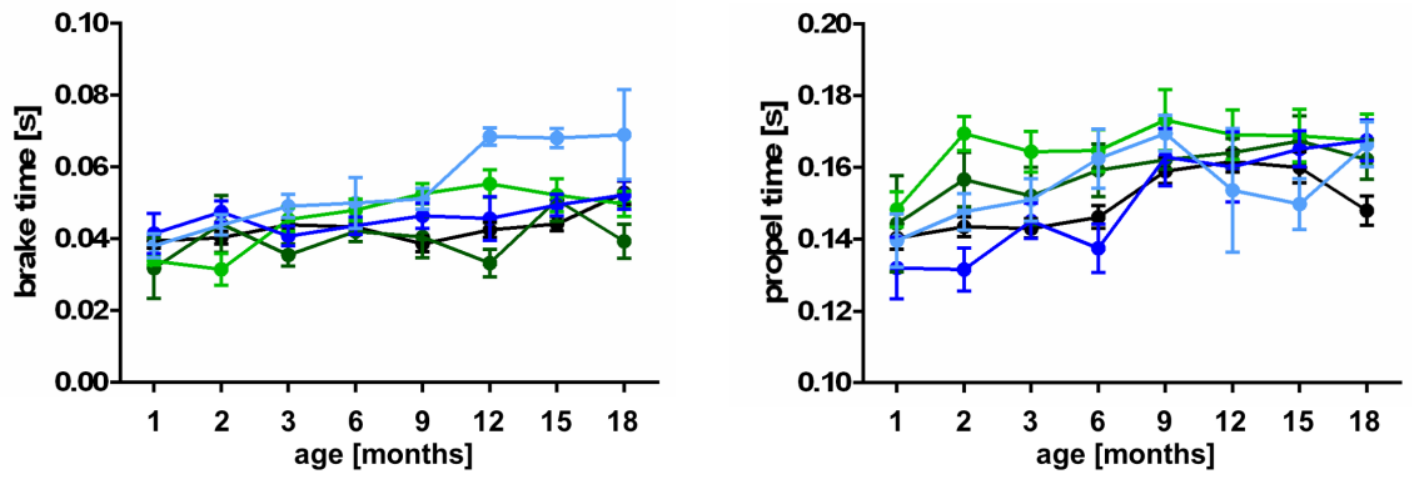

Supplemental Figure 1 - Hind limb gait rhythm in endophilin mutants. Gait timing parameters of WT (black), endophilin 1KO-2WT-3WT (dark green), 1KO-2HT-3WT (light green), 1KO-2WT-3KO (dark blue) and 1KO-2HT-3KO (light blue) animals recorded from the hind limbs on a DigiGait treadmill over the age of 1-18 months. (A) Stance time $=$ time the paw is in contact with the ground. (B) Swing time = time the paw is in the air. (C) Brake time = during stance, the time that the paw area is increasing. (D) Propel time = during stance, the time that the paw area is decreasing. In hind limbs, basic gait rhythm parameters are not changed in endophilin mutants. Running speed $=20 \mathrm{~cm} / \mathrm{s}$. Graphs show mean $\pm S E M$. Significance by Dunnettcorrected one-way ANOVA (compared to WT) is displayed in the tables above the charts. ${ }^{* * * *}=p<0.0001 ;{ }^{* * *}=p<0.001 ;{ }^{* *}=p<0.01 ;{ }^{*}=p<0.05$. 

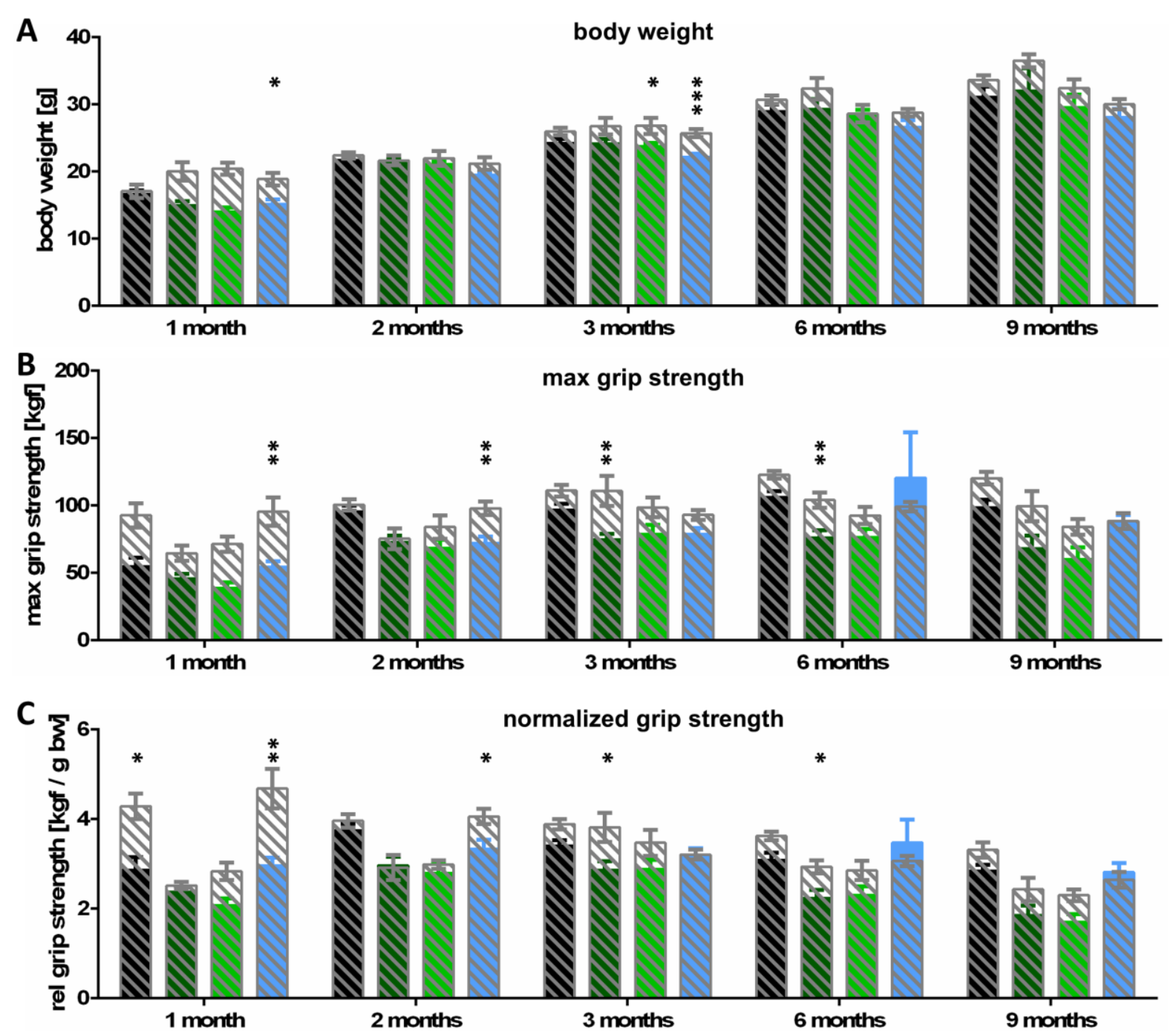

F32KO-E1WT-E2WT-E3WT

F32HT-E1KO-E2WT-E3WT $\square$ F32HT-E1KO-E2HT-E3WT

F32HT-E1KO-E2HT-E3KO

Supplemental Figure 2 - Weight and grip strength tend to decrease in Fbxo32 deficient endophilin mutants. (A) Body weight, (B) maximal grip strength and (C) normalized grip strength of Fbxo32 KO (black), Fbxo32 HT-endophilin 1KO-2WT3WT (dark green), Fbxo32 HT-endophilin 1KO-2HT-3WT (light green) and Fbxo32 $\mathrm{HT}$-endophilin 1KO-2HT-3KO (blue) animals. Values measured in animals with WT Fbxo32 levels and the corresponding endophilin genotype (as reported in Figure 23) are shown as grey dashed bars for comparison. Graphs show mean \pm SEM. Significance by Tukey-corrected one-way ANOVA compared to the corresponding endophilin mutant. ${ }^{* * *}=p<0.001 ;{ }^{* *}=p<0.01 ;{ }^{*}=p<0.05$. $\mathrm{kgf}=$ kilogram force 


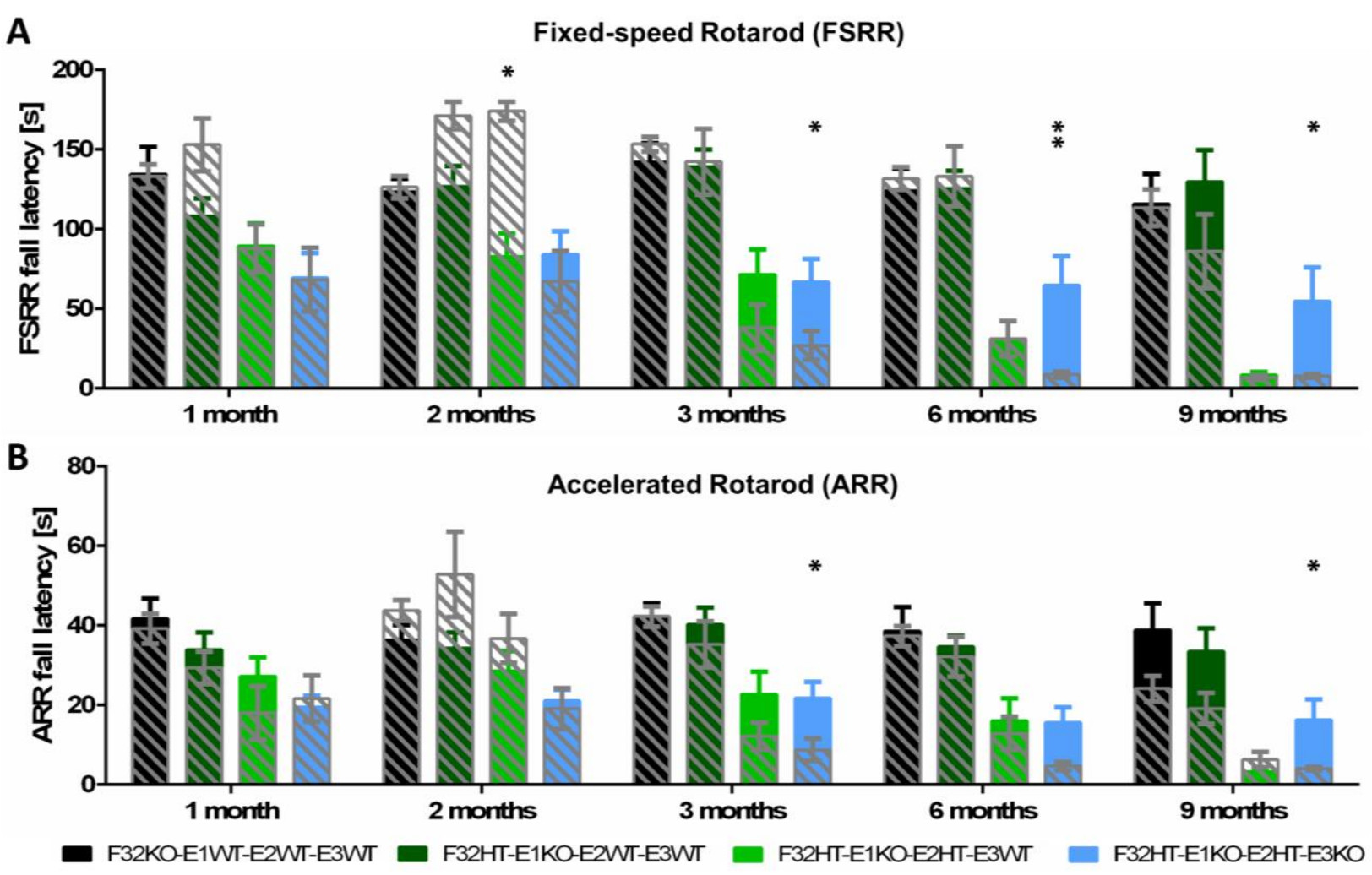

Supplemental Figure 3 - Rotarod performance is ameliorated upon genetic reduction of Fbxo32 in endophilin mutants. (A) Fall latency from the fixed speed RotaRod and (B) accelerated Rotarod of Fbxo32 KO (black), Fbxo32 HT-endophilin 1KO-2WT-3WT (dark green), Fbxo32 HT-endophilin 1KO-2HT-3WT (light green) and Fbxo32 HT-endophilin 1KO-2HT-3KO (blue) animals. Values measured in animals with WT Fbxo32 levels and the corresponding endophilin genotype (as reported in Figure 24) are shown as grey dashed bars for comparison. Graphs show mean \pm SEM. Significance by Tukey-corrected one-way ANOVA compared to the corresponding endophilin mutant. ${ }^{* *}=p<0.01 ;{ }^{*}=p<0.05$. 


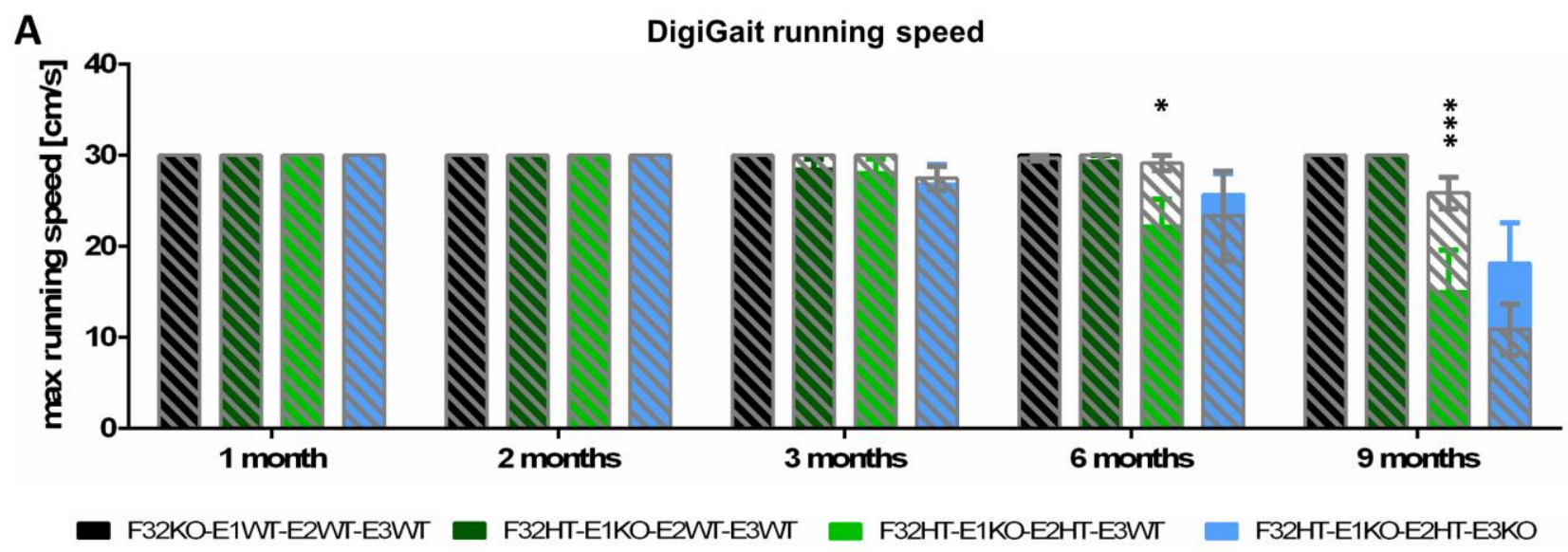

Supplemental Figure 4 - Maximal running speed of Fbxo32 deficient endophilin mutants. Average running speed of Fbxo32 KO (black), Fbxo32 HT-endophilin 1KO2WT-3WT (dark green), Fbxo32 HT-endophilin 1KO-2HT-3WT (light green) and Fbxo32 HT-endophilin 1KO-2HT-3KO (blue) animals. Values measured in animals with WT Fbxo32 levels and the corresponding endophilin genotype (as reported in Figure 25) are shown as grey dashed bars for comparison. Graphs show mean \pm SEM. Significance by Tukey-corrected one-way ANOVA compared to the corresponding endophilin mutant. ${ }^{* * *}=p<0.001 ;{ }^{*}=p<0.05$. 


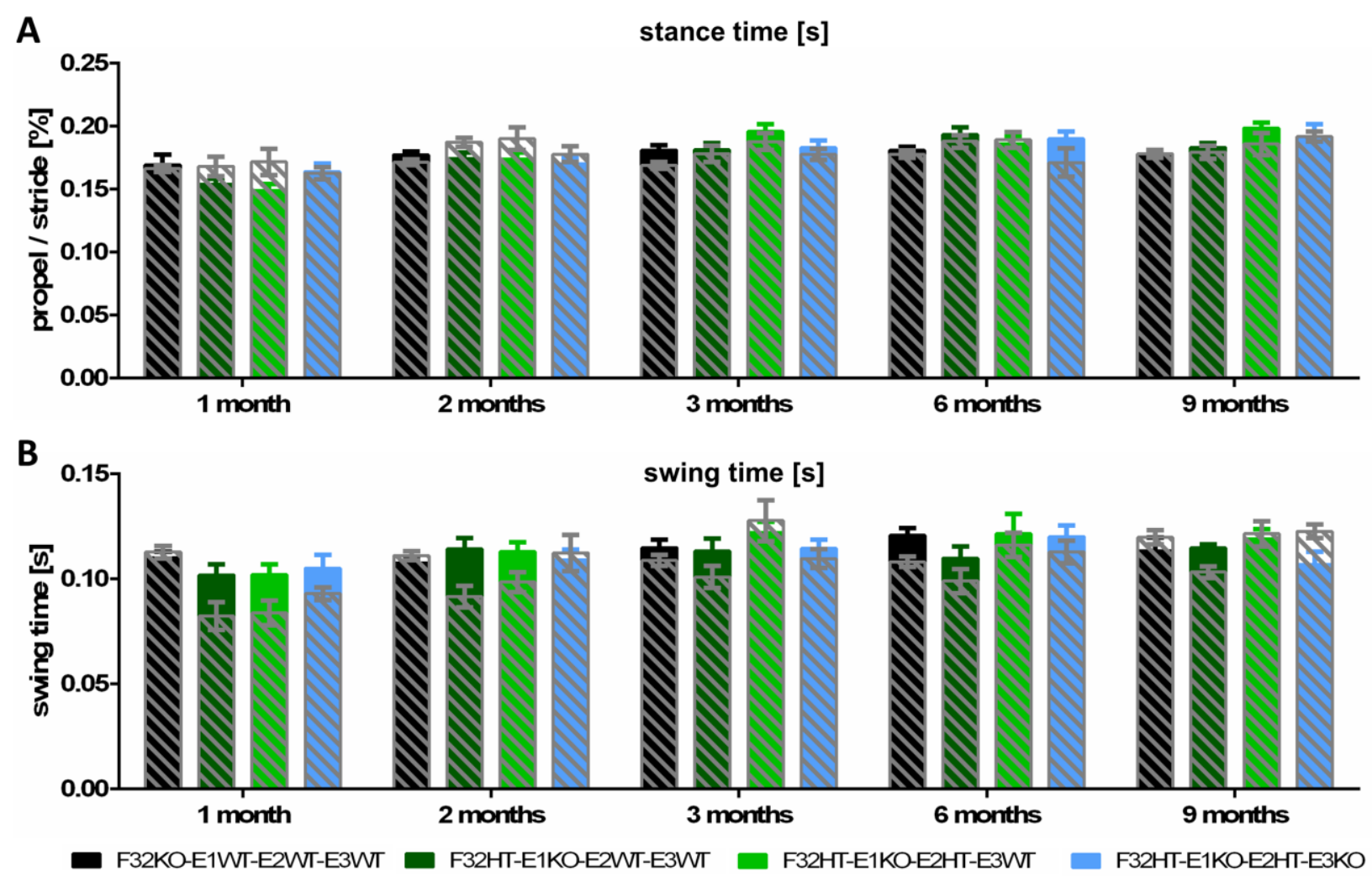

Supplemental Figure 5 - Fore limb gait rhythm is not affected in Fbxo32 deficient endophilin mutants. Gait timing parameters of Fbxo32 KO (black), Fbxo32 HTendophilin 1KO-2WT-3WT (dark green), Fbxo32 HT-endophilin 1KO-2HT-3WT (light green) and Fbxo32 HT-endophilin 1KO-2HT-3KO (blue) animals. Values measured in animals with WT Fbxo32 levels and the corresponding endophilin genotype (as reported in Figure 26) are shown as grey dashed bars for comparison. (A) Stance time = time the paw is in contact with the ground. (B) Swing time = time the paw is in the air. Running speed $=20 \mathrm{~cm} / \mathrm{s}$. Graphs show mean $\pm S E M$. No significance to the corresponding endophilin mutant was detected by Tukey-corrected one-way ANOVA compared. 


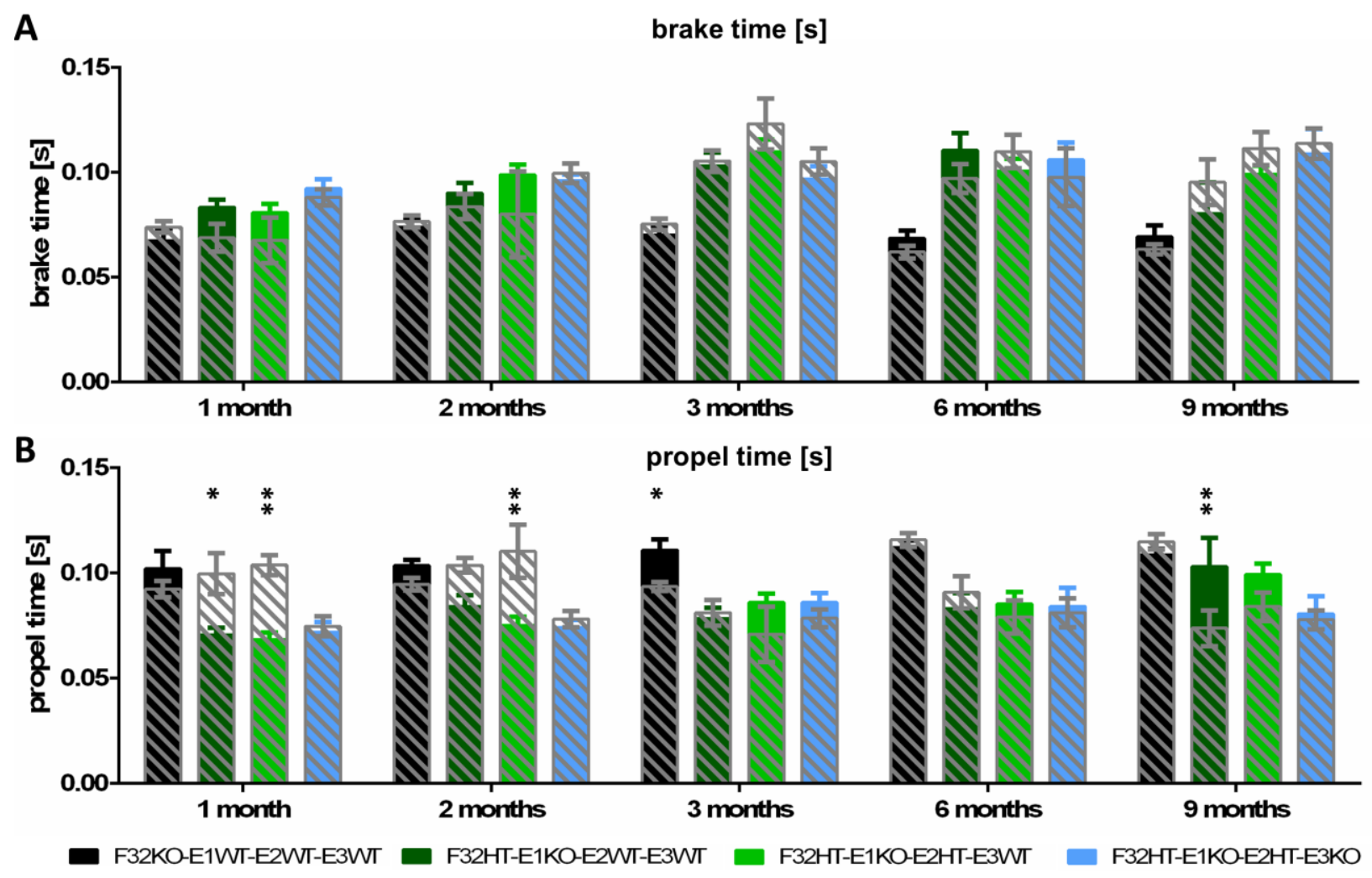

Supplemental Figure 6 - Fore limb stance rhythm tends to ameliorate in Fbxo32 deficient endophilin mutants. Gait timing parameters of Fbxo32 KO (black), Fbxo32 HT-endophilin 1KO-2WT-3WT (dark green), Fbxo32 HT-endophilin 1KO-2HT-3WT (light green) and Fbxo32 HT-endophilin 1KO-2HT-3KO (blue) animals. Values measured in animals with WT Fbxo32 levels and the corresponding endophilin genotype (as reported in Figure 26) are shown as grey dashed bars for comparison. (A) Brake time = during stance, the time that the paw area is increasing. (B) Propel time $=$ during stance, the time that the paw area is decreasing. Running speed $=20$ $\mathrm{cm} / \mathrm{s}$. Graphs show mean $\pm S E M$. Significance by Tukey-corrected one-way ANOVA compared to the corresponding endophilin mutant. ${ }^{* *}=p<0.01 ;{ }^{*}=p<0.05$. 


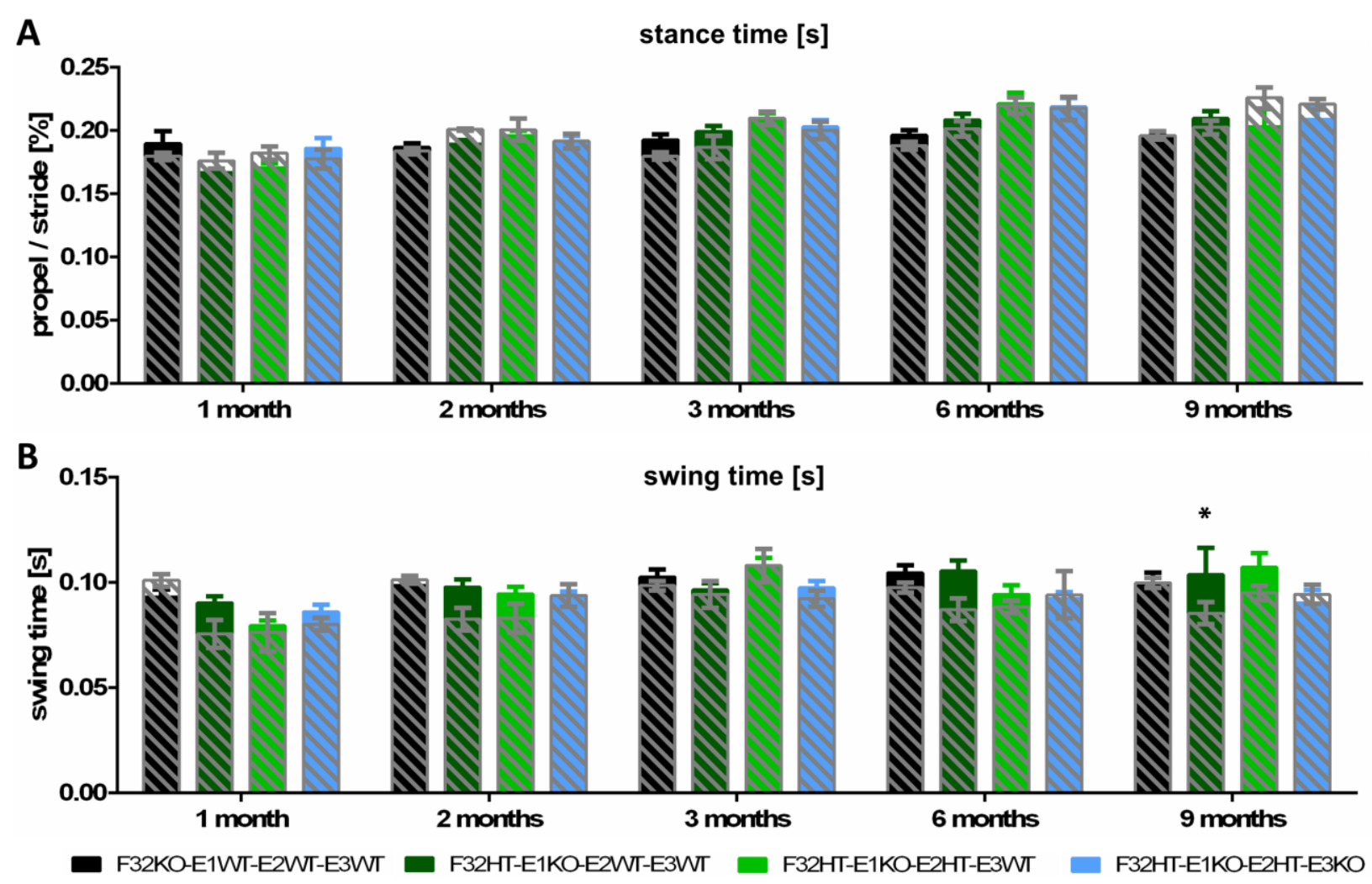

Supplemental Figure 7 - Hind limb gait rhythm in Fbxo32 deficient endophilin mutants. Gait timing parameters of Fbxo32 KO (black), Fbxo32 HT-endophilin 1KO2WT-3WT (dark green), Fbxo32 HT-endophilin 1KO-2HT-3WT (light green) and Fbxo32 HT-endophilin 1KO-2HT-3KO (blue) animals. Values measured in animals with WT Fbxo32 levels and the corresponding endophilin genotype (as reported in Supplemental Figure 1) are shown as grey dashed bars for comparison. (A) Stance time = time the paw is in contact with the ground. (B) Swing time = time the paw is in the air. Running speed $=20 \mathrm{~cm} / \mathrm{s}$. Graphs show mean $\pm S E M$. Significance by Tukeycorrected one-way ANOVA compared to the corresponding endophilin mutant. ${ }^{*}=p<0.05$. 


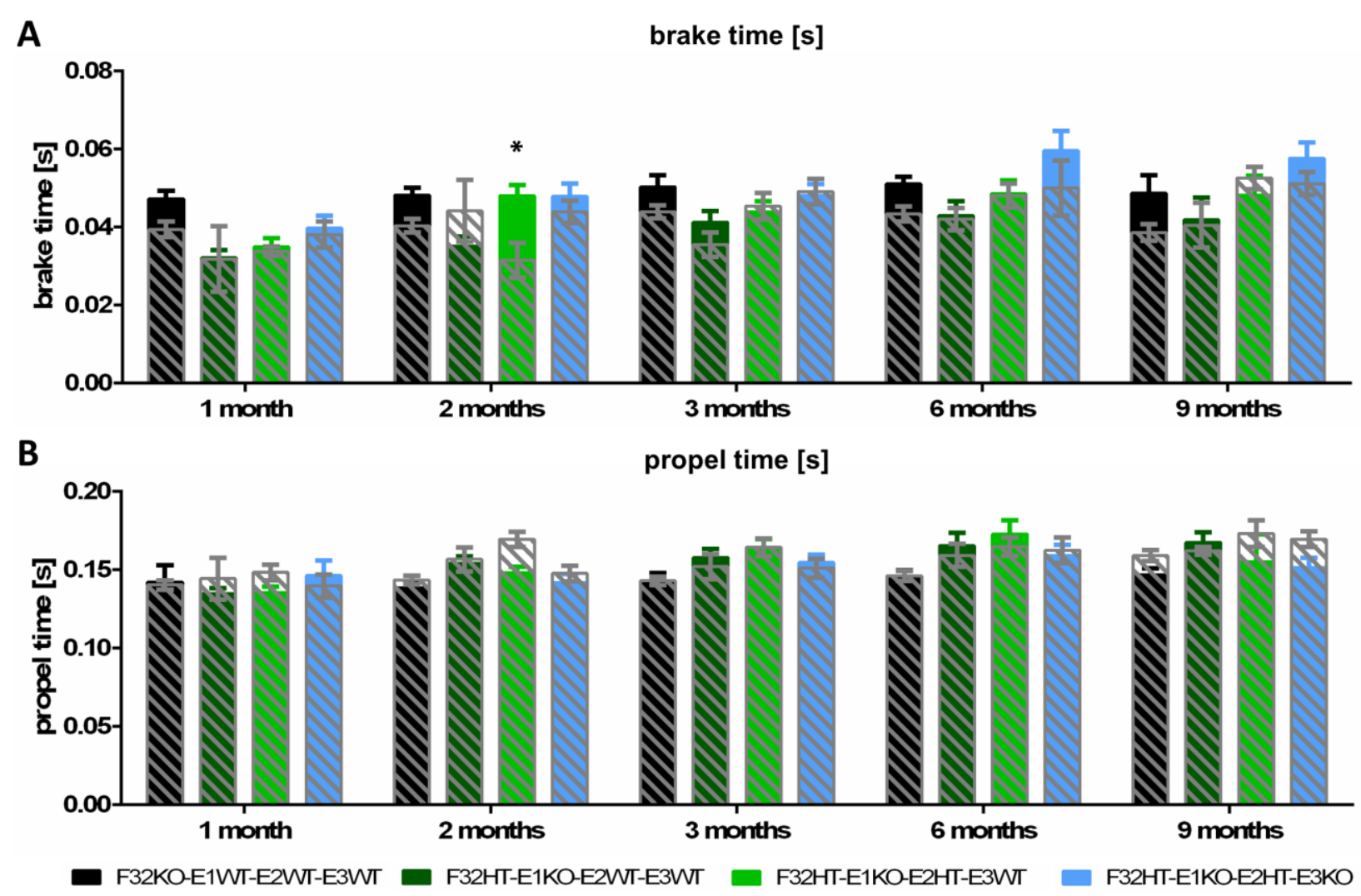

Supplemental Figure 8 - Hind limb stance rhythm in Fbxo32 deficient endophilin mutants. Gait timing parameters of Fbxo32 KO (black), Fbxo32 HT-endophilin 1KO2WT-3WT (dark green), Fbxo32 HT-endophilin 1KO-2HT-3WT (light green) and Fbxo32 HT-endophilin 1KO-2HT-3KO (blue) animals. Values measured in animals with WT Fbxo32 levels and the corresponding endophilin genotype (as reported in Supplemental Figure 1) are shown as grey dashed bars for comparison. (A) Brake time $=$ during stance, the time that the paw area is increasing. (B) Propel time $=$ during stance, the time that the paw area is decreasing. Running speed $=20 \mathrm{~cm} / \mathrm{s}$. Graphs show mean $\pm S E M$. Significance by Tukey-corrected one-way ANOVA compared to the corresponding endophilin mutant. ${ }^{*}=p<0.05$. 


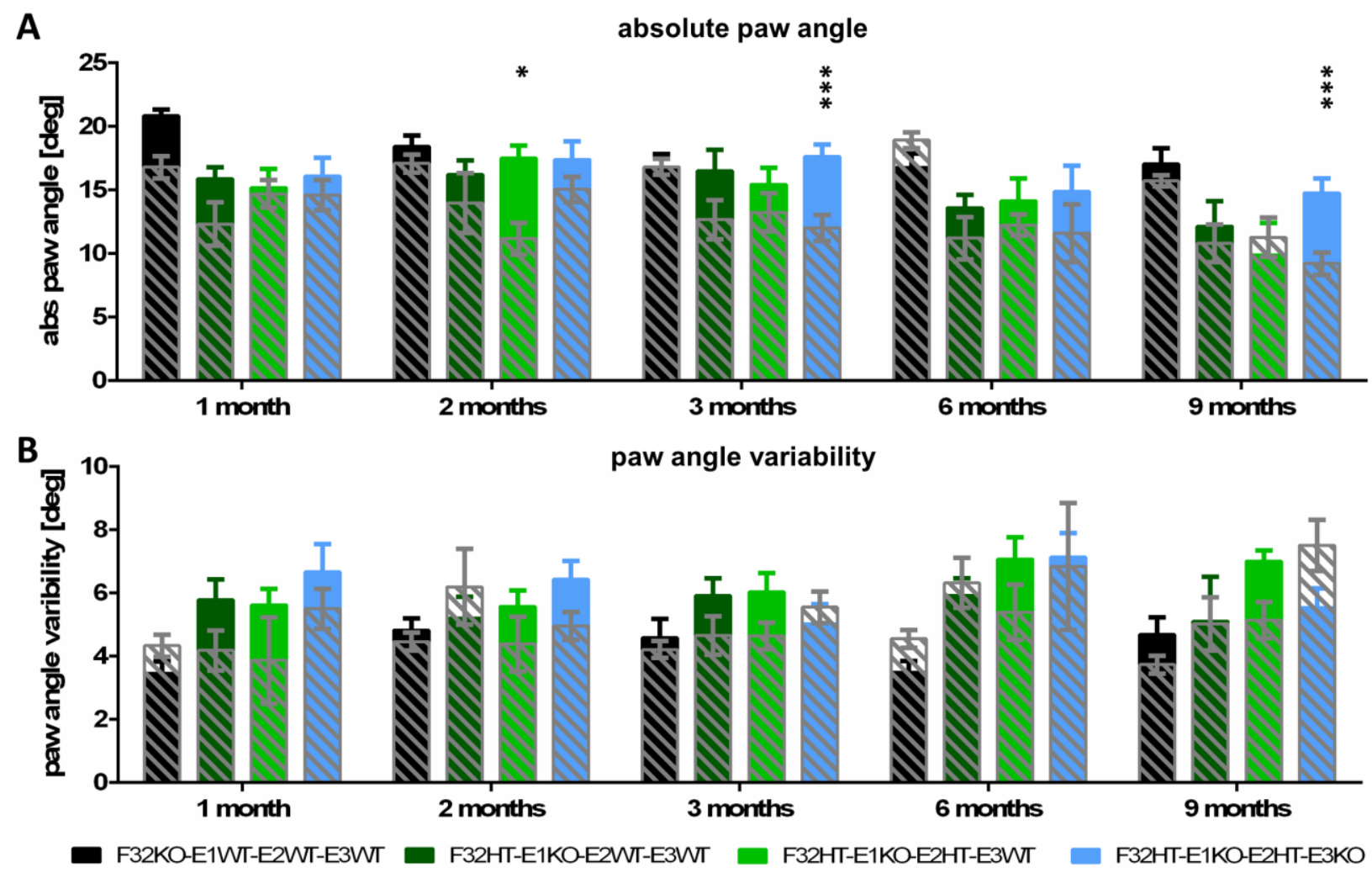

Supplemental Figure 9 - Fbxo32 deficiency in endophilin mutants ameliorates the paw angle during running. Hind limb paw placement parameters at $20 \mathrm{~cm} / \mathrm{s}$ for Fbxo32 KO (black), Fbxo32 HT-endophilin 1KO-2WT-3WT (dark green), Fbxo32 HTendophilin 1KO-2HT-3WT (light green) and Fbxo32 HT-endophilin 1KO-2HT-3KO (blue) animals. Values measured in animals with WT Fbxo32 levels and the corresponding endophilin genotype (as reported in Figure 27) are shown as grey dashed bars for comparison. (A) Paw angle = angle between the paw and the snouttail axis. (B) Paw angle variability = standard deviation of the paw angle. Graphs show mean $\pm S E M$. Significance by Tukey-corrected one-way ANOVA compared to the corresponding endophilin mutant. ${ }^{* * *}=p<0.001 ;{ }^{*}=p<0.05$. 


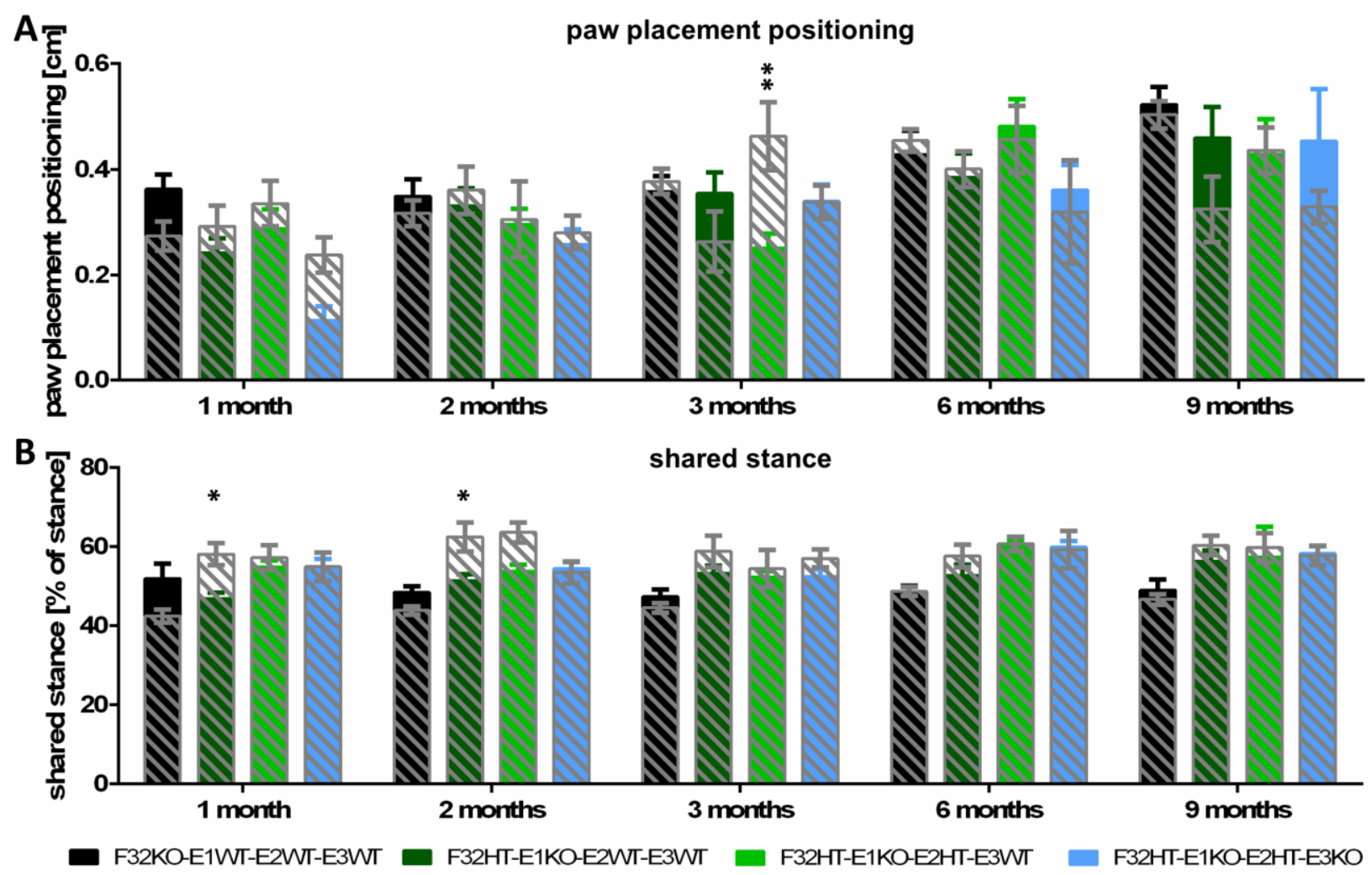

Supplemental Figure 10 - Fbxo32 deficiency in endophilin mutants tends to ameliorate posture during running. Hind $\operatorname{limb}$ posture parameters at $20 \mathrm{~cm} / \mathrm{s}$ for Fbxo32 KO (black), Fbxo32 HT-endophilin 1KO-2WT-3WT (dark green), Fbxo32 HTendophilin 1KO-2HT-3WT (light green) and Fbxo32 HT-endophilin 1KO-2HT-3KO (blue) animals. Values measured in animals with WT Fbxo32 levels and the corresponding endophilin genotype (as reported in Figure 27) are shown as grey dashed bars for comparison. (A) Paw placement positioning = extent of overlap between ipsilateral fore and hind paws during full stance. (B) Shared stance = time both hind limbs are in contact with the ground at the same time. Graphs show mean $\pm S E M$. Significance by Tukey-corrected one-way ANOVA compared to the corresponding endophilin mutant. ${ }^{* *}=p<0.01 ;{ }^{*}=p<0.05$. 


\section{List of figures}

Figure 1 Schematic representation of neuronal signal transduction ........................ 4

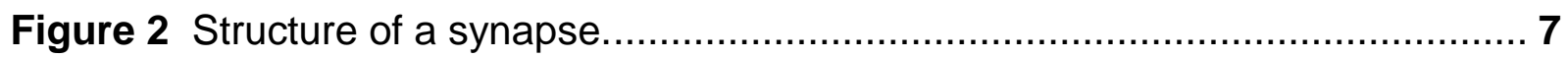

Figure 3 3D reconstruction of dendritic spines..................................................... 8

Figure 4 Clathrin mediated endocytosis ....................................................... 11

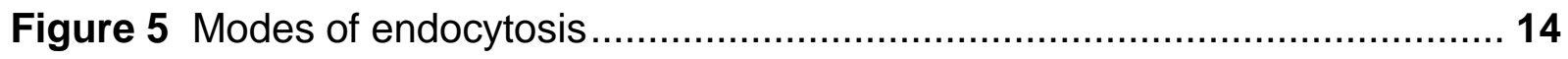

Figure 6 Crystal structure of an endophilin-A1 dimer......................................... 16

Figure 7 Accumulation in CCVs in endophilin deficient neurons........................... 21

Figure 8 Pathway analysis and upregulation of Fbxo32 in endophilin TKO brains .. 22

Figure 9 Accumulation of CCVs in synaptojanin-1 KO synaptic terminals ............... 23

Figure 10 Accumulation of CCPs in the dynamin 1,3 DKO synapse ..................... 24

Figure 11 Accumulation of CCVs in auxilin KO neurons....................................... 25

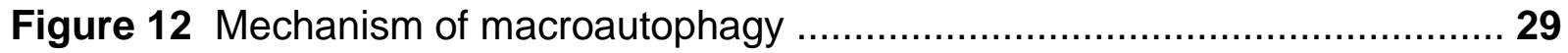

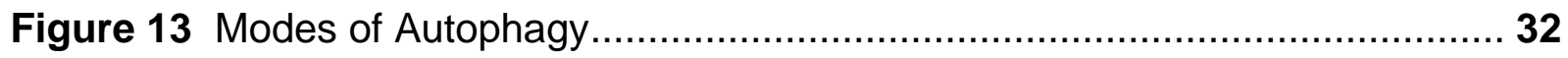

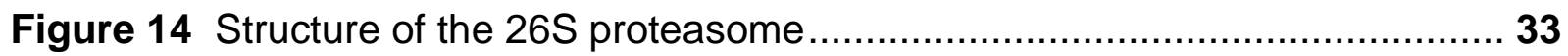

Figure 15 Substrate poly-ubiquitination through a RING protein or the SCF complex

Figure 16 Sequence and domain structure of Fbxo32 ….................................. 38

Figure 17 Expression of Fbxo32 in different tissues upon starvation..................... 40

Figure 18 Consequences of defective autophagy ........................................... 47

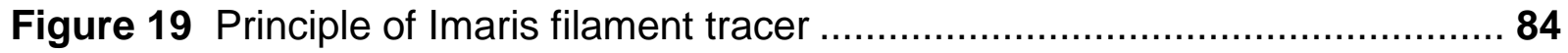

Figure 20 The endophilin mouse model ...................................................... 88

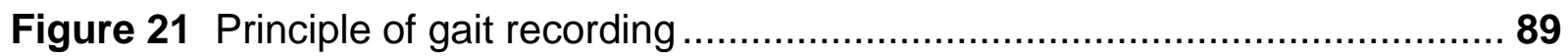

Figure 22 Gait parameters change in aging WT mice …................................... 90

Figure 23 Grip strength is reduced in endophilin mutants ................................... 91

Figure 24 Rotarod performance is impaired in endophilin mutants....................... 92

Figure 25 Running speed is reduced in endophilin mutants ................................ 93

Figure 26 Fore limb coordination is impaired in endophilin mutants ...................... 95

Figure 27 Posture parameters are changed in running endophilin mutants ............ 96

Figure 28 Motor behavior is unaffected in synaptojanin-1 deficient mice ............... 98 
Figure 29 Motor behavior is slightly impaired in dynamin-1,3 deficient mice .......... 99

Figure 30 Increase of apoptotic / necrotic cells in endophilin DKO brains ............. 101

Figure 31 Increase of apoptotic/necrotic cells in aged endophilin mutants ........... 102

Figure 32 Gliosis in the cortex of endophilin mutants ....................................... 103

Figure 33 Increased apoptotic activity in the endophilin TKO cortex .................... 104

Figure 34 No increase of apoptotic activity in synaptojanin-1 deficient cortex ....... 105

Figure 35 A-synuclein decreases in endophilin DKO brains and forms bigger puncta

Figure 36 Spine density and size are not changed in endophilin DKO mutants .... 107

Figure 37 Fbxo32 protein levels are increased in aged endophilin 1KO-2HT-3KO ..... brains 109

Figure 38 Foxo3A-P mislocalizes in endophilin TKO brains 110

Figure 39 Increase of poly-ubiquitinated proteins in endophilin DKO and TKO brains

Figure 40 Poly-ubiquitination is unaffected in synaptojanin and dynamin mutants $\mathbf{1 1 2}$ Figure 41 Atg5 protein levels are reduced in aged endophilin 1KO-2HT-3KO brains.

Figure 42 Levels of Atg9a and Atg16I are unchanged in endophilin mutants.... 115

Figure 43 Atg5 protein levels are unchanged in synaptojanin and dynamin mutants

Figure 44 Fbxo32 localizes to SV necks during endocytosis ............................ 117

Figure 45 Endophilin levels are normal in Fbxo32 KO brains ............................ 118

Figure 46 Genotyping for Fbxo32 deficient endophilin mutants.......................... 119

Figure 47 Weight and grip strength are decreased in Fbxo32 deficient endophilin .....

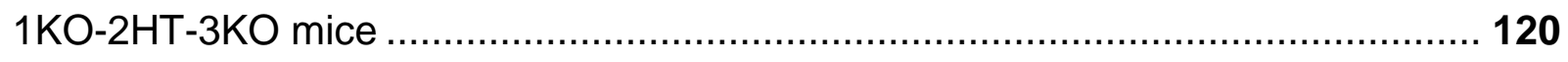

Figure 48 Rotarod performance is ameliorated upon genetic reduction of Fbxo32 in endophilin 1KO-2HT-3KO mice 121

Figure 49 Maximal running speed of Fbxo32 deficient endophilin 1KO-2HT-3KO mice

Figure 50 Fore limb gait rhythm is not affected in Fbxo32 deficient endophilin 1KO2HT-3KO mice.

Figure 51 Fbxo32 deficiency in endophilin 1KO-2HT-3KO mice ameliorates posture during running. 
Supplemental Figure 1 Hind limb gait rhythm in endophilin mutants..................... ii

Supplemental Figure 2 Weight and grip strength tend to decrease in Fbxo32

deficient endophilin mutants.

iii

Supplemental Figure 3 Rotarod performance is ameliorated upon genetic reduction of Fbxo32 in endophilin mutants. iv

Supplemental Figure 4 Maximal running speed of Fbxo32 deficient endophilin

mutants V

Supplemental Figure 5 Fore limb gait rhythm is not affected in Fbxo32 deficient ....... endophilin mutants vi

Supplemental Figure 6 Fore limb stance rhythm tends to ameliorate in Fbxo32 deficient endophilin mutants. vii Supplemental Figure 7 Hind limb gait rhythm in Fbxo32 deficient endophilin mutants viii

Supplemental Figure 8 Hind limb stance rhythm in Fbxo32 deficient endophilin mutants ix

Supplemental Figure 9 Fbxo32 deficiency in endophilin mutants ameliorates the ..... paw angle during running $\mathbf{x}$

Supplemental Figure 10 Fbxo32 deficiency in endophilin mutants tends to ameliorate posture during running. $\mathbf{x i}$ 


\section{List of tables}

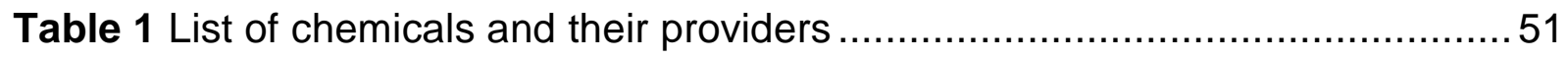

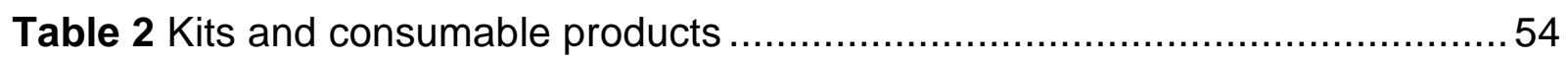

Table 3 Cell Culture consumables and equipment ...........................................56

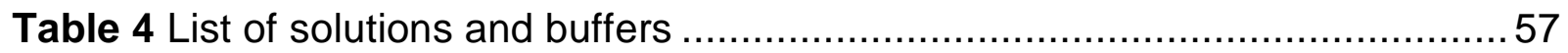

Table 5 Primary antibodies used for immunohistochemistry .............................. 59

Table 6 Secondary antibodies used for immunohistochemistry ............................59

Table 7 Primary antibodies used for western blot analysis ..................................6 60

Table 8 Secondary antibodies used for western blot analysis .............................61

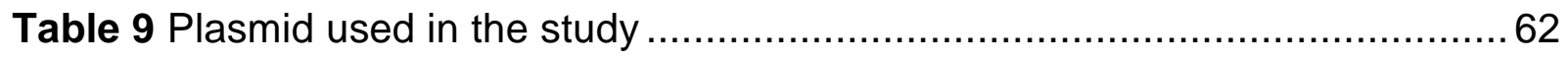

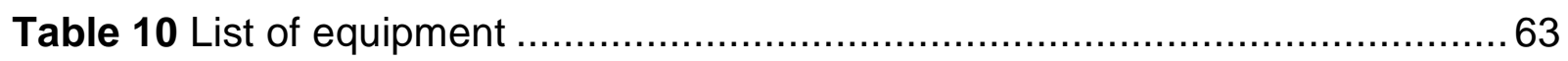

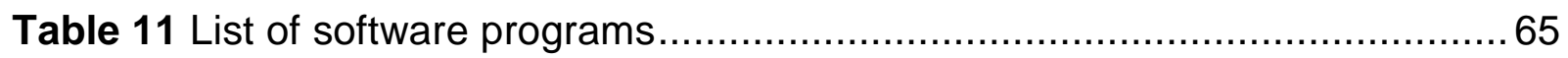

Table 12 Examples of gait parameters measured by DigiGait............................. 74

Table 13 Preperation of SDS gels (Volumes for $1 \mathrm{gel}$ with the Bio-Rad system) .... 85

Table 14 Summary of motor behavior parameters in endophilin mutants with WT and

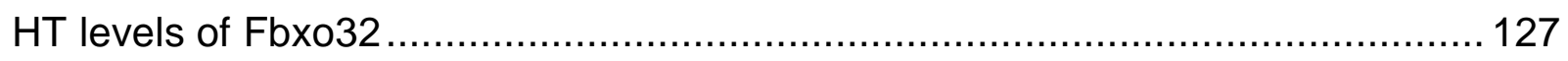




\section{Abbreviations}

aa

AD

ADBE

AMPK

AP2

ARR

ATG

ATP

BAR

BSA

CCP

$\mathrm{CCV}$

CHMP2B

CME

CNS

cTKO

DAPI

DEG

DKO

DMEM

DMSO

DNA

dNTP

EDTA

eMI

EMT

ESCRT

ER

FBS

Fbxo32

FSRR amino acid

Alzheimer's Disease

activity-dependent bulk endocytosis

AMP-activated protein kinase

adapter protein 2

accelerated Rotarod

autophagy related gene

adenosine triphosphate

BIN1/amphiphysin/Rvs167p

bovine serum albumine

clathrin coated pit

clathrin coated vesicle

charged multivesicular body protein $2 \mathrm{~B}$

clathrin mediated endocytosis

central nervous system

conditional triple knock-out

4',6-diamidino-2-phenylindole

differentially expressed gene

double knock-out

Dulbecco's Modified Eagle Medium

dimethyl sulfoxide

deoxyribonucleic acid

deoxyribonucleotide triphosphate

ethylenediaminetetraacetic acid

endosomal-microautophagy

epithelial to mesenchymal transition

endosomal sorting complex

endoplasmic reticulum

Fetal Bovine Serum

F-Box protein 32

fixed-speed Rotarod 
GABA

GAK

GFP

GS

$\mathrm{H}_{2} \mathrm{O}$

HD

HECT

HEPES

Hsc70

HT

kgf

$\mathrm{KI}$

$\mathrm{KO}$

LC3

LE

LRRK

LSM

LTD

LTP

$\mathrm{LZ}$

MAPK1

MEF

MPTP

mRNA

MVB

MyoD

NES

NGS

NLS

$\mathrm{O} / \mathrm{N}$

OCT

$\mathrm{OHT}$

p0/p14 etc. gamma-aminobutyric acid

cyclin-G-dependent kinase

green fluorescent protein

grip strength

water

Huntington's Disease

homologous to the E6AP carboxyl terminus

4-(2-hydroxyethyl)-1-piperazineethanesulfonic acid

heat shock cognate 70

heterozygous

kilogram force

knock-in

knock-out

light chain 3

late endosomes

Leucine-rich-repeat kinase 2

laser scanning microscope

long term depression

long term potentiation

leucine zipper domain

mitogen-activated protein kinase 1

mouse embryonic fibroblasts

1-methyl-4-phenyl-1,2,3,6-tetrahydropyridine

messenger RNA

multi-vesicular bodies

myoblast determination protein

nuclear export sequence

next generation sequencing

nuclear localization sequences

over night

optimum cutting temperature solution

4-hydroxytamoxifen

postnatal-day 0 / 14 
PAGE

PBS

PCR

PD

PE

PFA

$\mathrm{PH}$

PI

PRD

PSD

PVDF

qPCR

RING

RNA

ROS

Rpm

Rpn

Rpt

RT

SCF

SDS

$\mathrm{SH} 3$

SV

TBS

TEMED

TKO

TUNEL

UPS

UVRAG

WT polyacrylamide gel electrophoresis

phosphate buffered saline

polymerase chain reaction

Parkinson's Disease

phosphatidylethanolamine

paraformaldehyde

pleckstrin homology

phosphoinositide

proline-rich domain

post-synaptic density

Polyvinylidene difluoride

quantitative Polymerase-Chain-Reaction

really interesting new gene

ribonucleic acid

reactive oxygen species

rotations per minute

regulatory particle non-ATPase

regulatory particle triple-ATPase

room temperature

Skp1, Cullin1, F-box protein complex

sodium dodecyl sulfate

SRC Homology 3

synaptic vesicle

Tris-buffered saline

$\mathrm{N}, \mathrm{N}, \mathrm{N}, \mathrm{N}^{\prime}$-tetramethylethylenediamine

triple knock-out

2.3.8 TdT-mediated dUTP-biotin nick end labeling

Ubiquitin-Proteasome-System

ultraviolet irradiation resistant-associated gene

wild-type 


\section{List of publications}

\section{First and co-first author publications}

Murdoch, J. D., Rostosky, C. M., Gowrisankaran, S., Arora, A. S., Soukup, S. F., Vidal, R., Capece, V., Freytag, S., Fischer, A., Verstreken, P., Bonn, S., Raimundo, N., Milosevic, I. (2016). Endophilin-A Deficiency Induces the Foxo3a-Fbxo32 Network in the Brain and Causes Dysregulation of Autophagy and the Ubiquitin-Proteasome System. Cell Reports, 17(4), 1071-1086

Rostosky, C. M., \& Milosevic, I. (2018). Gait Analysis of Age-dependent Motor Impairments in Mice with Neurodegeneration. J. Vis. Exp., 136, e57752.

\section{Other publications}

Fiuza, M., Rostosky, C. M., Parkinson, G. T., Bygrave, A. M., Halemani, N., Baptista, M., Milosevic, I., Hanley, J. G. (2017). PICK1 regulates AMPA receptor endocytosis via direct interactions with AP2 $\alpha$-appendage and dynamin. $J$ Cell Biol., 216(10), 3323-3338 


\section{Acknowledgements}

This work would not have been possible without the help of several people around me. First and foremost, I want to thank Dr. Ira Milosevic for giving me the opportunity to perform my doctoral work in her laboratory. During my whole time at the ENI, I never lacked for materials and support and was given a luxurious amount of office and lab space. To this and to her constant encouragement to get the best out of myself, I am very grateful.

I also want to use this opportunity to thank Prof. Thomas Bayer and Prof. Tiago Outeiro for accepting to act as my thesis advisory committee. The discussions we had during those meetings very always helpful and inspiring. To Prof. Outeiro I am also grateful for providing me access to his Leica microscope. I also want to thank Prof. Dr. Nils Brose, Dr. Sonja Wojcik and Dr. Nuno Raimundo for agreeing to be on my extended examination board.

The scientific environment in Göttingen is very appealing for young scientist. I want to thank the whole Sensory and Motor Neuroscience Doctoral Program and especially Susann Müller and Patricia Räke-Kügler for the organization of beautiful retreats. These retreats have always given great opportunities to discuss science in a friendly environment. In addition, I want to sincerely thank all organizers of the GGNB, especially Christin Schröter, for helping with all the administration related with the grad school and for the organization of inspiring career impulse sessions.

Likewise, I want to thank Christiane, Sissi, Matthias and Tim for ensuring that all administrative and IT processes at ENI run as smoothly as possible. You have always had an open ear to all my problems, extending well beyond the scope of your respective jobs. Moreover, I want to thank Nils and Peter for reliable and prompt help with any questions concerning microscopes or image analysis.

A huge part of this thesis relies on the work with experimental animals. My most sincere thanks go to all the animal caretakers who work at the ZTE ENI, foremost to Tatiana, Jessica, Janis and Alissa. You have done great work as ambassadors to the animals' needs and helped me on a daily basis to manage all the animals used for this study. 
Though they do not understand it, I am also grateful to the mice themselves. I hope that from the data presented here and in the papers published from this work, the gain for our society will proof to justify the sacrifice of these experimental animals.

I understand science as a group effort. For this reason, I want to thank all collaborators that I have worked with during my PhD time. Specifically, I want to thank Prof. Patrik Verstreken, Prof. André Fischer, Prof. Stefan Bonn and Dr. Jonathan Hanley along with their lab members for the scientific exchange across the borders.

The most important collaborators are those closest to home. I want to thank all current and former members of the Milosevic and Raimundo labs. You all have formed a wonderful atmosphere to work in but also to pass time outside the lab. I want to especially thank Nuno, Sindhu, King, Aman and Siona for actively contributing to my project as well as Dirk and Melanie for excellent technical assistance.

Outside of the scientific world I am very grateful to a number of close friends who have supported me during my $\mathrm{PhD}$ or even longer than that. By joining me on festivals, parties and girls-nights-out, adventuring in the Harz, the nexus or on paper, dancing at weddings or just enjoying a quiet night competing about geography skills you all have provided the necessary and very welcome balance to my work in the lab. You have enriched my life in more than one way and I hope you will continue to do so for several years to come.

Most importantly, I want to express my gratitude to my family. To Elke for being available at any time and giving advice on every possible problem. To Suse, Jule and Lisa for being best friends as well as sisters, to Jule especially for expanding the family by two wonderful new members. And to Martina, Hans and Chris, my second family. For your understanding, your encouragement and the safe haven you continue to provide I will be eternally grateful.

The person I owe the most thanks to is my husband Matze. You have supported me on every single day of my $\mathrm{PhD}$ time, listened to my complaints and given me new perspectives on scientific problems. But most importantly, you have given me joy and happiness and created a wonderful home to return to after a long day in the lab. 